\title{
Modular Design of Chiral Conjugate-Base-Stabilized Carboxylic Acids: Catalytic Enantioselective [4+2] Cycloadditions of Acetals
}

\author{
Zhengbo Zhu, ${ }^{1}$ Minami Odagi, ${ }^{1,2}$ Nantamon Supantanapong, ${ }^{1}$ Weici Xu, ${ }^{1}$ Jaan Saame, ${ }^{3}$ Helmi-Ulrika \\ Kirm, ${ }^{3}$ Khalil A. Abboud, ${ }^{4}$ Ivo Leito, ${ }^{3}$ and Daniel Seidel ${ }^{1, *}$ \\ ${ }^{1}$ Center for Heterocyclic Compounds, Department of Chemistry, University of Florida, Gainesville, \\ Florida 32611, United States \\ ${ }^{2}$ Department of Biotechnology and Life Science, Graduate School of Technology, Tokyo University of \\ Agriculture and Technology, 2-24-16, Naka-cho, Koganei city, 184-8588, Tokyo, Japan \\ ${ }^{3}$ Institute of Chemistry, University of Tartu, Tartu, Estonia. \\ ${ }^{4}$ Center for X-ray Crystallography, Department of Chemistry, University of Florida, Gainesville, Florida \\ 32611, United States. \\ * Correspondence to: seidel@chem.ufl.edu
}

\section{Supporting Information}

\section{Table of Contents}

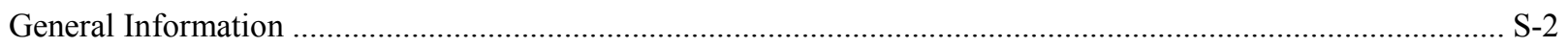

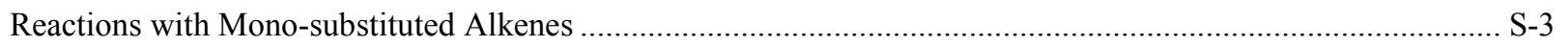

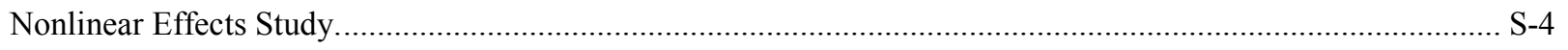

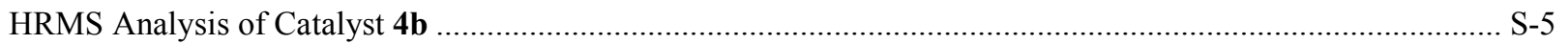

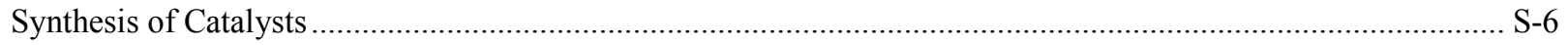

General Procedure for the Catalytic Enantioselective [4+2] Cycloaddition of Acetals .................................... S-24

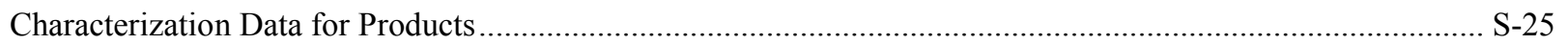

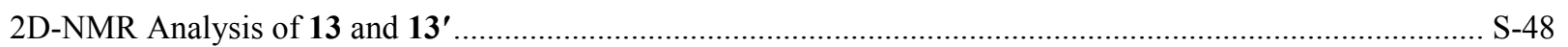

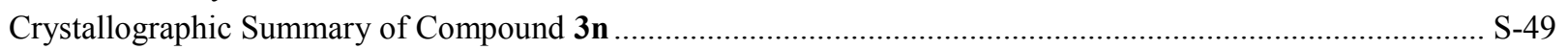

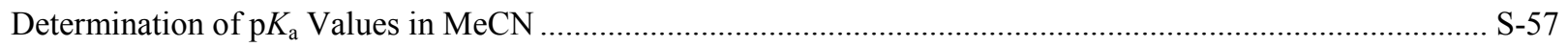

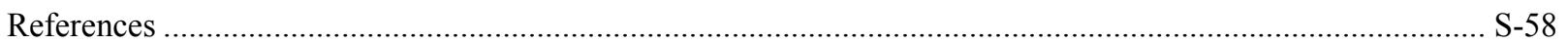

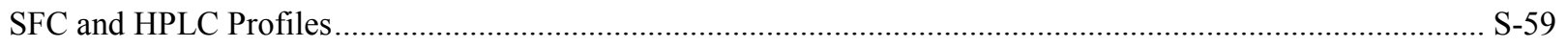

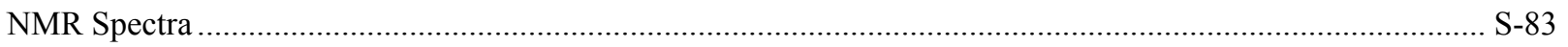


General Information: Reagents were purchased from commercial sources and were purified by distillation or recrystallization prior to use. Tetrahydrofuran (THF) and toluene (PhMe) were dried using a JC-Meyer solvent purification system. Purification of reaction products was carried out by flash column chromatography using Sorbent Technologies Standard Grade silica gel (60 A, 230-400 mesh) or neutral aluminium oxide (Brockmann I,

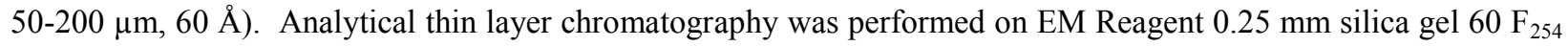
plates. Visualization was accomplished with UV light, phosphomolybdic acid and potassium permanganate stains, followed by heating. Proton nuclear magnetic resonance spectra ( $\left.{ }^{1} \mathrm{H}-\mathrm{NMR}\right)$ were recorded on Varian VNMRS-500 $\mathrm{MHz}$, Bruker $400 \mathrm{MHz}$ and $600 \mathrm{MHz}$ instruments, and chemical shifts are reported in ppm using the solvent as an internal standard $\left(\mathrm{CDCl}_{3}\right.$ at $7.26 \mathrm{ppm},\left(\mathrm{CD}_{3}\right)_{2} \mathrm{SO}$ at $\left.2.50 \mathrm{ppm}\right)$. Data are reported as app = apparent, $\mathrm{s}=$ singlet, $\mathrm{d}=$ doublet, $\mathrm{t}=$ triplet, $\mathrm{q}=$ quartet, $\mathrm{dd}=$ doublet of doublets, $\mathrm{td}=$ triplet of doublets, $\mathrm{dt}=$ doublet of triplets, $\mathrm{ddd}=$ doublet of doublet of doublets, dqd $=$ doublet of quartet of doublets, dddd = doublet of doublet of doublet of doublets, $\mathrm{m}=$ multiplet, comp = complex, $\mathrm{br}=$ broad; coupling constant(s) in Hz. Proton-decoupled carbon nuclear magnetic resonance spectra $\left({ }^{13} \mathrm{C}-\mathrm{NMR}\right)$ were recorded on Varian VNMRS-500 MHz, Bruker $400 \mathrm{MHz}$ and 600 $\mathrm{MHz}$ instruments and chemical shifts are reported in ppm using the solvent as an internal standard $\left(\mathrm{CDCl}_{3}\right.$ at 77.16 ppm, $\left(\mathrm{CD}_{3}\right)_{2} \mathrm{SO}$ at $\left.39.52 \mathrm{ppm}\right)$. High resolution mass spectra (HRMS) were obtained from the Mass Spectrometry Core Laboratory of the University of Florida (Agilent 6200 ESI-TOF coupled to an IonSense DART ET-100 ionization source for DART-TOF analysis). HPLC analysis was carried out on an Agilent 1100 series instrument with autosampler and multiple wavelength detectors. Supercritical fluid chromatography (SFC) analysis was carried out on an Agilent 1260 Infinity II series instrument with auto sampler and multiple wavelength detectors. Optical rotations were measured using a $1 \mathrm{~mL}$ cell with a $1 \mathrm{dm}$ path length on a Jasco $\mathrm{P}-2000$ polarimeter at $589 \mathrm{~nm}$ and at $20{ }^{\circ} \mathrm{C}$. Salicylaldehyde dimethyl acetals were prepared according to established procedures. ${ }^{1}$ Alcohols $\mathbf{1 c},{ }^{2} \mathbf{1 d}{ }^{3}$ $1 \mathrm{e},{ }^{4} \mathbf{6},{ }^{5} \mathbf{8},{ }^{5}$ and $12^{6}$ were prepared according to literature procedures and their published characterization data matched with our own in all respects. The relative configurations of $7,{ }^{5} \mathbf{9},{ }^{5}$ and $\mathbf{1 1} \mathbf{1}^{7}$ were confirmed based on literature data. 


\section{Reactions with Mono-substituted Alkenes. ${ }^{a}$}

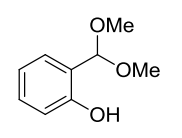

$1 a$

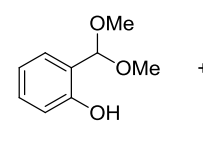

1a

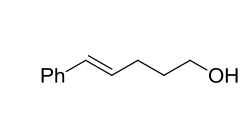

S1 (1.2 equiv)

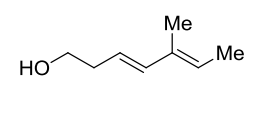

S3 (1.2 equiv)
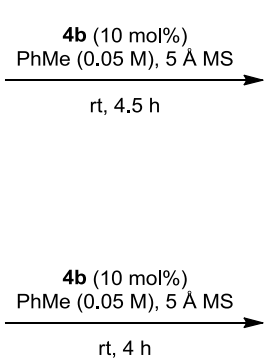

$\pi, 4 \mathrm{~h}$

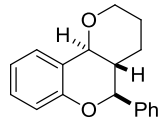

S2, $83 \%, 31 \%$ ee

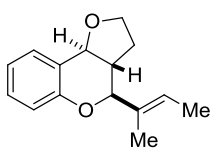

S4, $15 \%$,

$\mathrm{dr}=2: 1$,

ee $=34 \% / 33 \%$

${ }^{\text {a }}$ Reactions were performed with $0.25 \mathrm{mmol}$ of $\mathbf{1 a}$ and 1.2 equiv of alcohols. Yields correspond to isolated product. 


\section{Nonlinear Effects Study}

Following general procedure $\mathrm{C}$ for the enantioselective [4+2] cycloadditions of acetals (see below), the reaction was conducted using catalyst $\mathbf{4 b}$ with $0 \%, 20 \%, 40 \%, 60 \%, 80 \%,>99 \%$ ee at room temperature. A notable positive nonlinear effect was observed in the formation of $\mathbf{3 a}$.

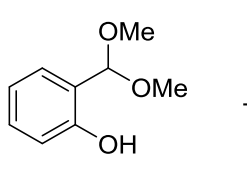

$1 \mathrm{a}$

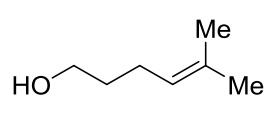

2a (1.2 equiv)
PhMe (0.05 M), 5 A MS, rt, 30 min

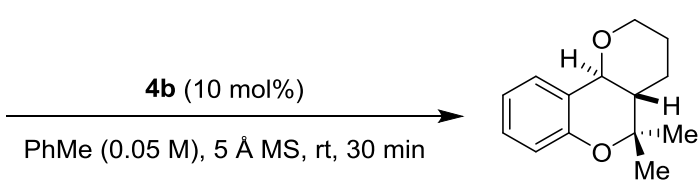

$3 a$

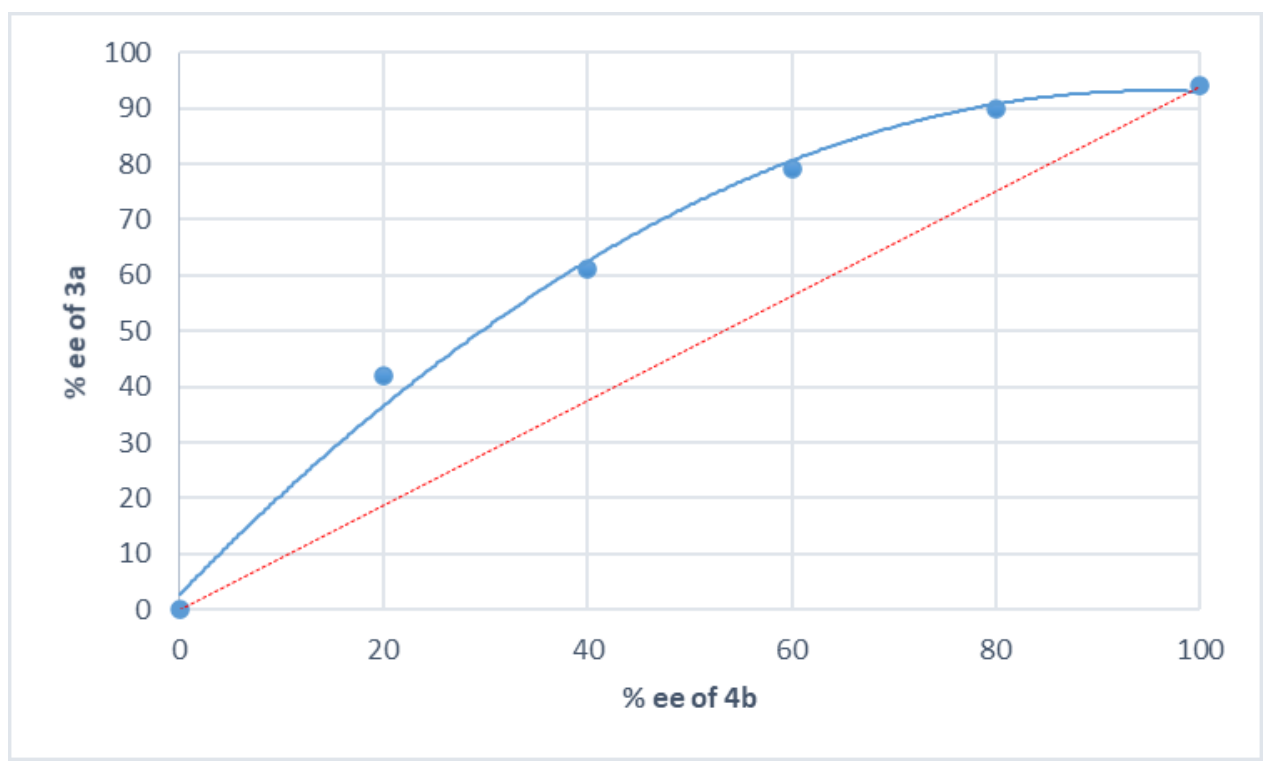

Figure S1. Nonlinear effects study for the formation of product $\mathbf{3 a}$ with catalyst $\mathbf{4 b}$. 


\section{HRMS Analysis of Catalyst $4 b$}

Electrospray Ionization Mass Spectrometry (ESI-MS) studies were performed to gauge the potential of catalyst $\mathbf{4 b}$ to undergo aggregation. At negative ionization mode, in addition to the peak at $\mathrm{m} / \mathrm{z} 848.7370$ which corresponds to the anion of the catalyst $\mathbf{4 b}$, another peak at $\mathrm{m} / \mathrm{z} 1698.4778$ is observed, consistent with a dimeric catalyst species.

\section{Spectrum Plot Report _ Agilent}

\begin{tabular}{|c|c|c|c|c|c|c|c|}
\hline Name & negmode_38_negESI & Rack Pos. & & Instrument & Instrument 1 & Operator & \\
\hline Inj. Vol. (ul) & & Plate Pos. & & IRM Status & Some ions missed & & \\
\hline Data File & negmode_38_negESI.d & Method (Acq) & SS_Negative-mode.m & Comment & & Acq. Time (Local) & $\begin{array}{l}\text { 6/16/2020 12:49:38 } \\
\text { PM (UTC-04:00) }\end{array}$ \\
\hline
\end{tabular}

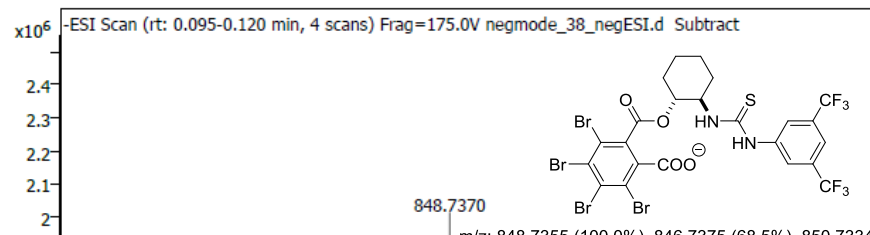

m/z: 848.7355 (100.0\%), 846.7375 (68.5\%), 850.7334 (64.9\%)

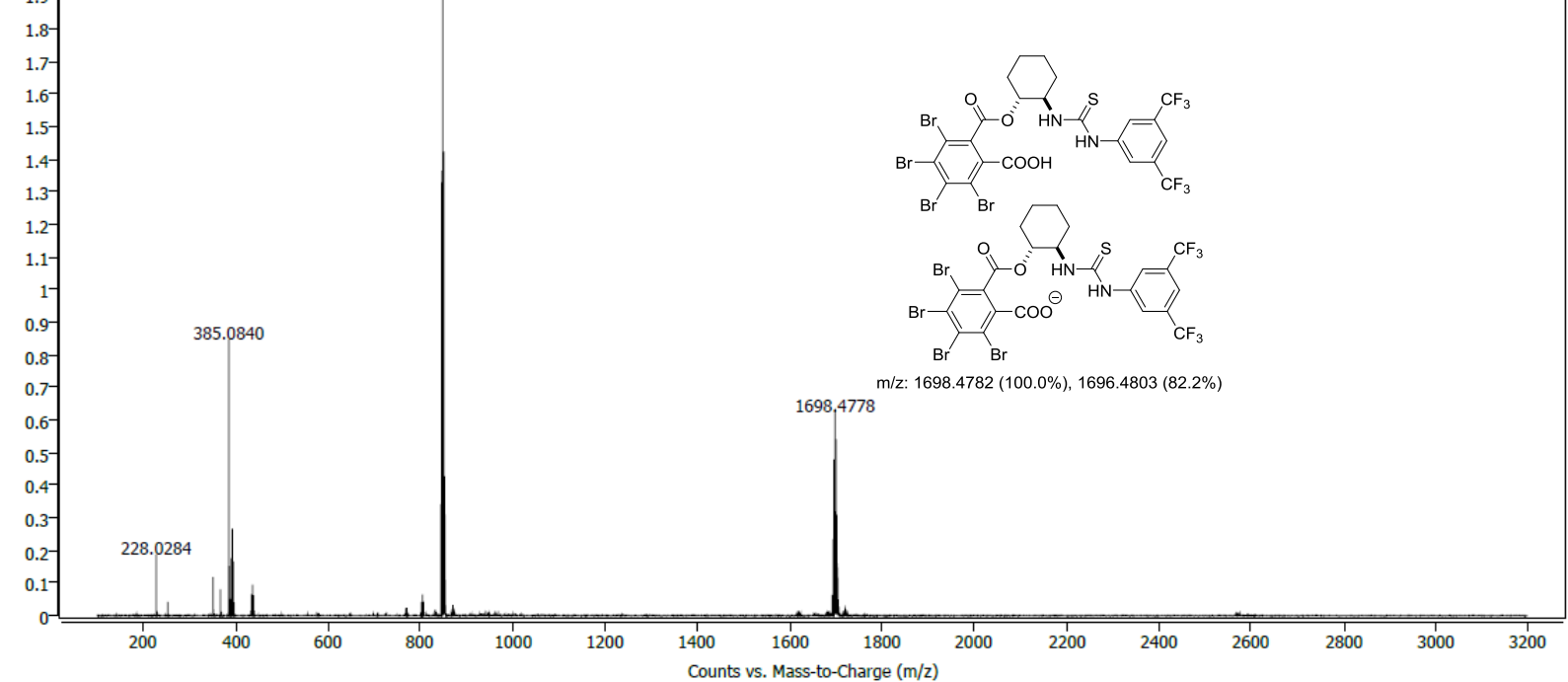

Figure S2. ESI-MS analysis of catalyst $\mathbf{4 b}$. 


\section{Synthesis of Catalysts}

\section{General Procedure A:}

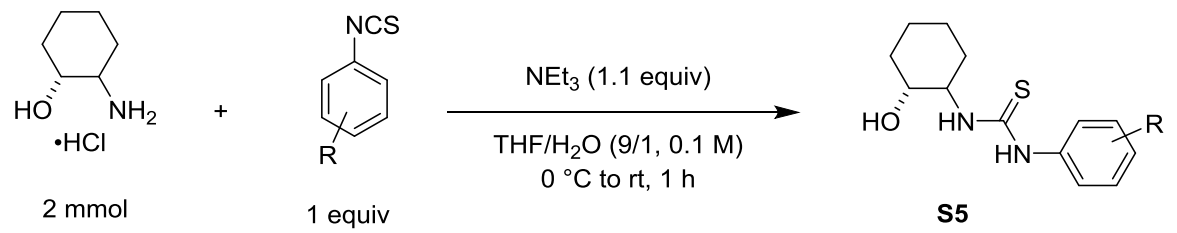

To an ice cooled solution of the amino alcohol hydrochloride $(2 \mathrm{mmol})$ in $\mathrm{THF} / \mathrm{H}_{2} \mathrm{O}(9: 1,18 \mathrm{~mL} / 2 \mathrm{~mL})$ was added triethylamine ( $307 \mu \mathrm{L}, 2.2 \mathrm{mmol}, 1.1$ equiv), followed by isothiocyanate $(2 \mathrm{mmol}, 1$ equiv). The resulting mixture was then allowed to warm to room temperature and stirred for $1 \mathrm{~h}$, then transferred to a separatory funnel. EtOAc $(30 \mathrm{~mL})$ was added and the mixture washed with $1 \mathrm{M} \mathrm{HCl}(30 \mathrm{~mL} \times 2)$. The combined aqueous layers were extracted with EtOAc $(30 \mathrm{~mL} \times 2)$, and the combined organic layers washed with brine $(40 \mathrm{~mL})$ and dried over anhydrous $\mathrm{Na}_{2} \mathrm{SO}_{4}$. Solvent was then removed under reduced pressure and the residue purified by silica gel chromatography (using 20\% EtOAc in hexanes as eluent). 


\section{1-(3,5-Bis(trifluoromethyl)phenyl)-3-((1R,2R)-2-hydroxycyclohexyl)thiourea}

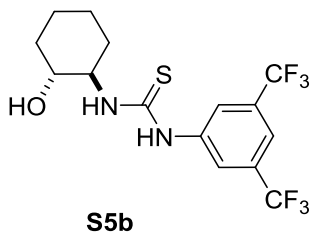

To an ice cooled solution of $(1 R, 2 R)$-2-aminocyclohexan-1-ol hydrochloride $(1.5 \mathrm{~g}, 9.9 \mathrm{mmol})$ in $\mathrm{THF} / \mathrm{H}_{2} \mathrm{O}(9: 1,36$ $\mathrm{mL} / 4 \mathrm{~mL}$ ) was added triethylamine $(1.52 \mathrm{~mL}, 10.9 \mathrm{mmol}, 1.1$ equiv), followed by 3,5-bis(trifluoromethyl)phenyl isothiocyanate $(1.81 \mathrm{~mL}, 9.9 \mathrm{mmol}, 1$ equiv). The resulting mixture was then allowed to warm to room temperature and stirred for $1 \mathrm{~h}$, then transferred to a separatory funnel. EtOAc $(100 \mathrm{~mL})$ was added and the mixture washed with $1 \mathrm{M} \mathrm{HCl}(100 \mathrm{~mL} \times 2)$. The combined aqueous layers were extracted with EtOAc $(100 \mathrm{~mL} \times 2)$, and the combined organic layers washed with brine $(120 \mathrm{~mL})$ and dried over anhydrous $\mathrm{Na}_{2} \mathrm{SO}_{4}$. Solvent was then removed under reduced pressure and the residue purified by silica gel chromatography (using 20\% EtOAc in hexanes as eluent) to provide $\mathbf{S 5 b}$ as a white solid in $95 \%$ yield (3.64 g).

\section{Characterization data for S5b:}

$\mathbf{R}_{\mathbf{f}}=0.40$ in hexanes $/$ EtOAc $50: 50 \mathrm{v} / \mathrm{v}$.

$[\boldsymbol{\alpha}]_{\mathbf{D}}^{20}=-35.82\left(\mathrm{c} 0.5, \mathrm{CHCl}_{3}\right)$.

${ }^{1} \mathrm{H}-\mathrm{NMR}\left(+,\left(\mathrm{CD}_{3}\right)_{2} \mathrm{SO}\right): \delta=9.93(\mathrm{~s}, 1 \mathrm{H}), 8.24(\mathrm{~s}, 2 \mathrm{H}), 8.17(\mathrm{~s}, 1 \mathrm{H}), 7.68(\mathrm{~s}, 1 \mathrm{H}), 4.78-4.65(\mathrm{~m}, 1 \mathrm{H}), 4.06-3.88(\mathrm{~m}$, $1 \mathrm{H}), 3.47-3.35(\mathrm{~m}, 1 \mathrm{H}), 2.24-2.03(\mathrm{~m}, 1 \mathrm{H}), 1.96-1.85(\mathrm{~m}, 1 \mathrm{H}), 1.73-1.51$ (comp, 2H), 1.36-1.00 (comp, 4H).

${ }^{13}$ C-NMR $\left(150 \mathrm{MHz},\left(\mathrm{CD}_{3}\right)_{2} \mathrm{SO}\right): \delta=179.8,142.1,130.2\left(\mathrm{q}, J_{C-F}=33.3 \mathrm{~Hz}\right), 123.3\left(\mathrm{q}, J_{C-F}=272.7 \mathrm{~Hz}\right), 121.4$, 115.6, 70.8, 59.3, 34.3, 29.9, 24.0, 23.7.

${ }^{19}$ F-NMR (565 MHz, $\left.\left(\mathrm{CD}_{3}\right)_{2} \mathrm{SO}\right): \delta=-61.57$.

HRMS (ESI-TOF): Calculated for $\mathrm{C}_{15} \mathrm{H}_{17} \mathrm{~F}_{6} \mathrm{~N}_{2} \mathrm{OS}[\mathrm{M}+\mathrm{H}]^{+}: 387.0960$, found 387.0961. 


\section{1-(3,5-Bis(trifluoromethyl)phenyl)-3-((1R,2S)-2-hydroxycyclohexyl)thiourea}

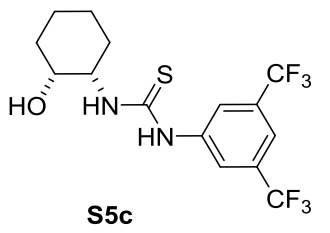

Following general procedure A, compound $\mathbf{S 5 c}$ was obtained from $(1 R, 2 S)$-2-aminocyclohexan-1-ol hydrochloride (303 $\mathrm{mg}, 2 \mathrm{mmol}, 1$ equiv) and 3,5-bis(trifluoromethyl)phenyl isothiocyanate $(0.365 \mathrm{~mL}, 2 \mathrm{mmol}, 1$ equiv) as a white solid in $96 \%$ yield $(745 \mathrm{mg})$.

\section{Characterization data for S5c:}

$\mathbf{R}_{\mathbf{f}}=0.54$ in hexanes/EtOAc 50:50 v/v.

$[\boldsymbol{\alpha}]_{\mathbf{D}}^{20}=+27.36\left(\mathrm{c} 0.5, \mathrm{CHCl}_{3}\right)$

${ }^{1}$ H-NMR $\left(600 \mathrm{MHz},\left(\mathrm{CD}_{3}\right)_{2} \mathrm{SO}\right): \delta=10.28(\mathrm{~s}, 1 \mathrm{H}), 8.34(\mathrm{~s}, 2 \mathrm{H}), 7.89(\mathrm{~d}, J=8.1 \mathrm{~Hz}, 1 \mathrm{H}), 7.70(\mathrm{~s}, 1 \mathrm{H}), 5.02-4.87$ (m, 1H), 4.25-4.07 (m, 1H), 3.94-3.80 (m, 1H), 1.81-1.44 (comp, 6H), 1.36-1.21 (comp, 2H).

${ }^{13}$ C-NMR $\left(150 \mathrm{MHz},\left(\mathrm{CD}_{3}\right)_{2} \mathrm{SO}\right): \delta=179.0,142.0,130.1\left(\mathrm{q}, J_{C-F}=32.8 \mathrm{~Hz}\right), 123.2\left(\mathrm{q}, J_{C-F}=272.6 \mathrm{~Hz}\right), 121.3$, $115.8,66.2,55.2,32.0,25.8,23.9,19.1$.

${ }^{19}$ F-NMR $\left.\left(565 \mathrm{MHz},\left(\mathrm{CD}_{3}\right)\right)_{2} \mathrm{SO}\right): \delta=-61.59$.

HRMS (ESI-TOF): Calculated for $\mathrm{C}_{15} \mathrm{H}_{17} \mathrm{~F}_{6} \mathrm{~N}_{2} \mathrm{OS}[\mathrm{M}+\mathrm{H}]^{+}: 387.0960$, found 387.0956. 


\section{1-(4-Bromo-3,5-bis(trifluoromethyl)phenyl)-3-((1R,2R)-2-hydroxycyclohexyl)thiourea}

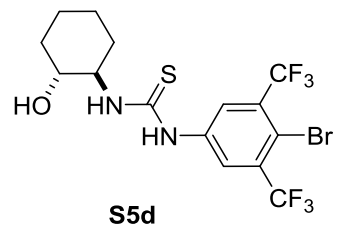

Following general procedure A, compound S5d was obtained from $(1 R, 2 R)$-2-aminocyclohexan-1-ol hydrochloride (303 mg, $2 \mathrm{mmol}, 1$ equiv) and 2-bromo-5-isothiocyanato-1,3-bis(trifluoromethyl)benzene (700 mg, $2 \mathrm{mmol}, 1$ equiv) as a white solid in $96 \%$ yield ( $895 \mathrm{mg}$ ).

\section{Characterization data for S5d:}

$\mathbf{R}_{\mathbf{f}}=0.33$ in hexanes/EtOAc 50:50 v/v.

$[\boldsymbol{\alpha}]_{\mathbf{D}}^{20}=-31.98\left(\mathrm{c} 0.5, \mathrm{CHCl}_{3}\right)$.

${ }^{1}$ H-NMR $\left(600 \mathrm{MHz},\left(\mathrm{CD}_{3}\right)_{2} \mathrm{SO}\right): \delta=9.99(\mathrm{~s}, 1 \mathrm{H}), 8.35(\mathrm{~s}, 2 \mathrm{H}), 8.27-8.15(\mathrm{~m}, 1 \mathrm{H}), 4.80-4.60(\mathrm{~m}, 1 \mathrm{H}), 4.05-3.85$ (m, 1H), 3.45-3.33 (m, 1H), 2.19-2.06 (m, 1H), 1.94-1.86 (m, 1H), 1.72-1.52 (comp, 2H), 1.33-1.00 (comp, 4H).

${ }^{13}$ C-NMR $\left(150 \mathrm{MHz},\left(\mathrm{CD}_{3}\right)_{2} \mathrm{SO}\right): \delta=179.5,140.5,130.8\left(\mathrm{q}, J_{C-F}=32.0 \mathrm{~Hz}\right), 123.6,122.5\left(\mathrm{q}, J_{C-F}=274.0 \mathrm{~Hz}\right)$, $108.9,70.8,59.4,34.3,29.9,24.0,23.7$.

${ }^{19}$ F-NMR (565 MHz, $\left.\left(\mathrm{CD}_{3}\right)_{2} \mathrm{SO}\right): \delta=-56.30$.

HRMS (ESI-TOF): Calculated for $\mathrm{C}_{15} \mathrm{H}_{16}{ }^{79} \mathrm{BrF}_{6} \mathrm{~N}_{2} \mathrm{OS}[\mathrm{M}+\mathrm{H}]^{+}$: 465.0065, found 465.0061; calculated for $\mathrm{C}_{15} \mathrm{H}_{16}{ }^{81} \mathrm{BrF}_{6} \mathrm{~N}_{2} \mathrm{OS}[\mathrm{M}+\mathrm{H}]^{+}: 467.0045$, found 467.0044 . 
1-(4-Cyano-3,5-bis(trifluoromethyl)phenyl)-3-((1R,2R)-2-hydroxycyclohexyl)thiourea

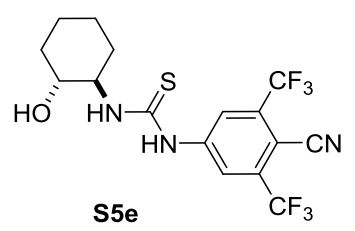

Following general procedure A, compound S5e was obtained from $(1 R, 2 R)$-2-aminocyclohexan-1-ol hydrochloride (303 mg, 2 mmol, 1 equiv) and 4-isothiocyanato-2,6-bis(trifluoromethyl)benzonitrile (592 mg, $2 \mathrm{mmol}, 1$ equiv) as a white solid in $98 \%$ yield $(810 \mathrm{mg})$.

\section{Characterization data for S5e:}

$\mathbf{R}_{\mathbf{f}}=0.23$ in hexanes $/$ EtOAc $50: 50 \mathrm{v} / \mathrm{v}$.

$[\boldsymbol{\alpha}]_{\mathbf{D}}^{20}=-26.84\left(\mathrm{c} 0.5, \mathrm{CHCl}_{3}\right)$.

${ }^{1}$ H-NMR $\left(600 \mathrm{MHz},\left(\mathrm{CD}_{3}\right)_{2} \mathrm{SO}\right): \delta=10.47(\mathrm{~s}, 1 \mathrm{H}), 8.68(\mathrm{~d}, J=7.8 \mathrm{~Hz}, 1 \mathrm{H}), 8.48(\mathrm{~s}, 2 \mathrm{H}), 4.84-4.66(\mathrm{~m}, 1 \mathrm{H}), 4.00-$ $3.89(\mathrm{~m}, 1 \mathrm{H}), 3.46-3.36(\mathrm{~m}, 1 \mathrm{H}), 2.17-2.07(\mathrm{~m}, 1 \mathrm{H}), 1.97-1.87(\mathrm{~m}, 1 \mathrm{H}), 1.72-1.55$ (comp, 2H), 1.33-1.15 (comp, $3 \mathrm{H}), 1.12-1.03(\mathrm{~m}, 1 \mathrm{H})$.

${ }^{13}$ C-NMR $\left(150 \mathrm{MHz},\left(\mathrm{CD}_{3}\right)_{2} \mathrm{SO}\right): \delta=179.0,145.7,134.0\left(\mathrm{q}, J_{C-F}=32.2 \mathrm{~Hz}\right), 122.0\left(\mathrm{q}, J_{C-F}=274.4 \mathrm{~Hz}\right), 119.8$, $112.6,96.7,70.7,59.8,34.4,29.6,24.0,23.8$.

${ }^{19}$ F-NMR $\left(565 \mathrm{MHz},\left(\mathrm{CD}_{3}\right)_{2} \mathrm{SO}\right): \delta=-56.49$.

HRMS (ESI-TOF): Calculated for $\mathrm{C}_{16} \mathrm{H}_{15} \mathrm{~F}_{6} \mathrm{~N}_{3} \mathrm{NaOS}[\mathrm{M}+\mathrm{Na}]^{+}: 434.0732$, found 434.0735. 


\section{1-((1R,2R)-2-hydroxycyclohexyl)-3-(4-nitro-3-(trifluoromethyl)phenyl)thiourea}

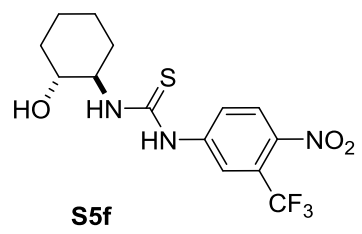

Following general procedure A, compound S5f was obtained from $(1 R, 2 R)$-2-aminocyclohexan-1-ol hydrochloride (303 mg, 2 mmol, 1 equiv) and 4-isothiocyanato-1-nitro-2-(trifluoromethyl)benzene (496 mg, $2 \mathrm{mmol}, 1$ equiv) as a yellow solid in $96 \%$ yield $(701 \mathrm{mg})$.

\section{Characterization data for S5f:}

$\mathbf{R}_{\mathbf{f}}=0.17$ in hexanes/EtOAc $50: 50 \mathrm{v} / \mathrm{v}$.

$[\boldsymbol{\alpha}]_{\mathbf{D}}^{20}=-28.76\left(\mathrm{c} 0.5, \mathrm{CHCl}_{3}\right)$.

${ }^{1}$ H-NMR $\left(600 \mathrm{MHz},\left(\mathrm{CD}_{3}\right)_{2} \mathrm{SO}\right): \delta=10.20(\mathrm{~s}, 1 \mathrm{H}), 8.45-8.33(\mathrm{comp}, 2 \mathrm{H}), 8.14(\mathrm{~d}, J=8.9 \mathrm{~Hz}, 1 \mathrm{H}), 8.00-7.88(\mathrm{~m}$, $1 \mathrm{H}), 4.80-4.71(\mathrm{~m}, 1 \mathrm{H}), 4.01-3.88(\mathrm{~m}, 1 \mathrm{H}), 3.47-3.35(\mathrm{~m}, 1 \mathrm{H}), 2.20-2.04(\mathrm{~m}, 1 \mathrm{H}), 1.95-1.85(\mathrm{~m}, 1 \mathrm{H}), 1.71-1.54$ (comp, 2H), 1.35-1.17 (comp, 3H), 1.15-1.01 (m, 1H).

${ }^{13}$ C-NMR $\left(150 \mathrm{MHz},\left(\mathrm{CD}_{3}\right)_{2} \mathrm{SO}\right): \delta=179.3,145.1,140.5,127.3,123.4,122.5\left(\mathrm{q}, J_{C-F}=33.9 \mathrm{~Hz}\right), 122.1\left(\mathrm{q}, J_{C-F}=\right.$ $272.9 \mathrm{~Hz}), 118.6,70.7,59.5,34.3,29.8,23.9,23.7$.

${ }^{19}$ F-NMR (565 MHz, $\left.\left(\mathrm{CD}_{3}\right)_{2} \mathrm{SO}\right): \delta=-54.23$.

HRMS (ESI-TOF): Calculated for $\mathrm{C}_{14} \mathrm{H}_{16} \mathrm{~F}_{3} \mathrm{~N}_{3} \mathrm{NaO}_{3} \mathrm{~S}[\mathrm{M}+\mathrm{Na}]^{+}: 386.0757$, found 386.0752. 


\section{1-((1R,2R)-2-hydroxycyclohexyl)-3-(3-(trifluoromethyl)phenyl)thiourea}

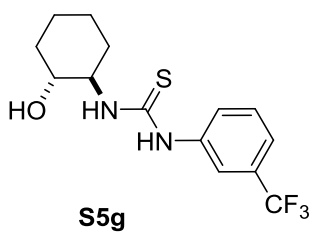

Following general procedure A, compound S5g was obtained from $(1 R, 2 R)$-2-aminocyclohexan-1-ol hydrochloride (303 mg, $2 \mathrm{mmol}, 1$ equiv) and 3-(trifluoromethyl)phenyl isothiocyanate $(0.304 \mathrm{~mL}, 2 \mathrm{mmol}, 1$ equiv) as a white solid in $97 \%$ yield $(621 \mathrm{mg})$.

\section{Characterization data for S5g:}

$\mathbf{R}_{\mathbf{f}}=0.31$ in hexanes/EtOAc $50: 50 \mathrm{v} / \mathrm{v}$.

$[\boldsymbol{\alpha}]_{\mathbf{D}}^{20}=-32.92\left(\mathrm{c} 0.5, \mathrm{CHCl}_{3}\right)$.

${ }^{1}$ H-NMR (600 MHz, $\left.\left(\mathrm{CD}_{3}\right)_{2} \mathrm{SO}\right): \delta=9.68(\mathrm{~s}, 1 \mathrm{H}), 8.06(\mathrm{~s}, 1 \mathrm{H}), 7.90(\mathrm{~s}, 1 \mathrm{H}), 7.68$ (app d, $\left.J=8.1 \mathrm{~Hz}, 1 \mathrm{H}\right), 7.51$ (app $\mathrm{t}, J=8.0 \mathrm{~Hz}, 1 \mathrm{H}), 7.38($ app d, $J=7.7 \mathrm{~Hz}, 1 \mathrm{H}), 4.83-4.60(\mathrm{~m}, 1 \mathrm{H}), 4.10-3.78(\mathrm{~m}, 1 \mathrm{H}), 3.44-3.34(\mathrm{~m}, 1 \mathrm{H}), 2.25-$ $2.06(\mathrm{~m}, 1 \mathrm{H}), 1.94-1.84(\mathrm{~m}, 1 \mathrm{H}), 1.70-1.52$ (comp, 2H), 1.35-0.99 (comp, 4H).

${ }^{13}$ C-NMR $\left(150 \mathrm{MHz},\left(\mathrm{CD}_{3}\right)_{2} \mathrm{SO}\right): \delta=179.9,140.7,129.5,129.0\left(\mathrm{q}, J_{C-F}=31.4 \mathrm{~Hz}\right), 125.5,124.1\left(\mathrm{q}, J_{C-F}=272.2\right.$ $\mathrm{Hz}), 119.6,118.1,70.9,59.1,34.3,30.1,23.9,23.7$.

${ }^{19}$ F-NMR (565 MHz, $\left.\left(\mathrm{CD}_{3}\right)_{2} \mathrm{SO}\right): \delta=-56.45$.

HRMS (ESI-TOF): Calculated for $\mathrm{C}_{14} \mathrm{H}_{18} \mathrm{~F}_{3} \mathrm{~N}_{2} \mathrm{OS}[\mathrm{M}+\mathrm{H}]^{+}: 319.1086$, found 319.1085 . 


\section{General Procedure B}

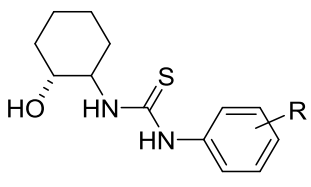

S5

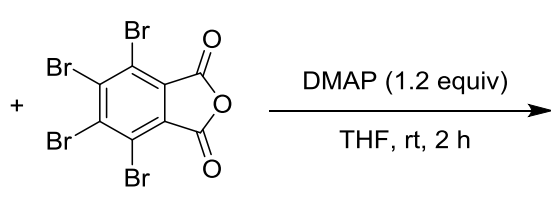

1.2 equiv

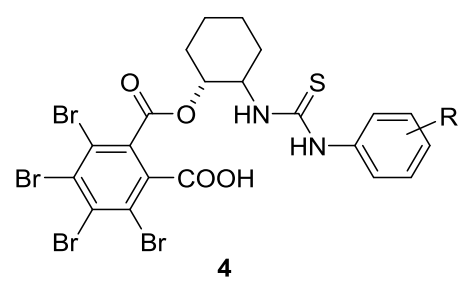

4

To a solution of thiourea alcohol S5 $(1.5 \mathrm{mmol})$ in THF $(15 \mathrm{~mL})$ were added $4-N, N$-dimethylaminopyridine (DMAP) (220 mg, $1.8 \mathrm{mmol}, 1.2$ equiv) and tetrabromophthalic anhydride ( $835 \mathrm{mg}, 1.8 \mathrm{mmol}, 1.2$ equiv). The resulting mixture was stirred at room temperature for $2 \mathrm{~h}$ and then transferred to a separatory funnel. EtOAc (100 mL) was added and the solution washed with $1 \mathrm{M} \mathrm{HCl}(50 \mathrm{~mL} \times 2)$, saturated aqueous $\mathrm{NaHCO}_{3}(50 \mathrm{~mL})$, and $1 \mathrm{M} \mathrm{HCl}(50$ $\mathrm{mL}$ ), and dried over anhydrous $\mathrm{Na}_{2} \mathrm{SO}_{4}$. The solvent was removed under reduced pressure and the residue purified by neutral $\mathrm{Al}_{2} \mathrm{O}_{3}$ chromatography (using a gradient eluent from $\mathrm{CH}_{2} \mathrm{Cl}_{2}$ to $\mathrm{MeOH} / \mathrm{CH}_{2} \mathrm{Cl}_{2}$ 10:90 v/v to $\mathrm{MeOH} / \mathrm{CH}_{2} \mathrm{Cl}_{2} 20: 80 \mathrm{v} / \mathrm{v}$ to $\mathrm{MeOH} / \mathrm{CH}_{2} \mathrm{Cl}_{2} / \mathrm{HCOOH}$ 20:79:1 v/v/v). The combined fractions were concentrated and re-dissolved in $\mathrm{CH}_{2} \mathrm{Cl}_{2}(50 \mathrm{~mL})$ and then washed with $1 \mathrm{M} \mathrm{HCl}(25 \mathrm{~mL} \times 2)$. The combined aqueous layers were back-extracted with $\mathrm{CH}_{2} \mathrm{Cl}_{2}(2 \times 25 \mathrm{~mL})$. The combined organic layers were dried over anhydrous $\mathrm{Na}_{2} \mathrm{SO}_{4}$. Finally, the solvent was removed under reduced pressure and the resulting solid dried under high vacuum. 
2-((((1R,2R)-2-(3-(3,5-bis(trifluoromethyl)phenyl)thioureido)cyclohexyl)oxy)carbonyl)-3,4,5,6tetrabromobenzoic acid

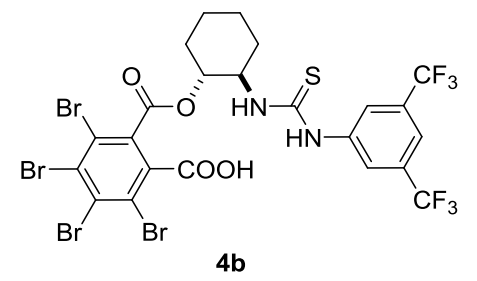

To a solution of thiourea alcohol S5b $(3.09 \mathrm{~g}, 8 \mathrm{mmol})$ in THF $(100 \mathrm{~mL})$ were added 4- $N, N$-dimethylaminopyridine (DMAP) (1.17 g, $9.6 \mathrm{mmol}, 1.2$ equiv) and tetrabromophthalic anhydride ( $4.45 \mathrm{~g}, 9.6 \mathrm{mmol}, 1.2$ equiv). The resulting mixture was stirred at room temperature for $1.5 \mathrm{~h}$ and then transferred to a separatory funnel. EtOAc (300 $\mathrm{mL}$ ) was added and the solution washed with $1 \mathrm{M} \mathrm{HCl}(100 \mathrm{~mL} \times 2)$, saturated aqueous $\mathrm{NaHCO}_{3}(100 \mathrm{~mL})$, and 1 $\mathrm{M} \mathrm{HCl}(100 \mathrm{~mL})$, and dried over anhydrous $\mathrm{Na}_{2} \mathrm{SO}_{4}$. The solvent was removed under reduced pressure and the residue purified by neutral $\mathrm{Al}_{2} \mathrm{O}_{3}$ chromatography (using a gradient eluent from $\mathrm{CH}_{2} \mathrm{Cl}_{2}$ to $\mathrm{MeOH} / \mathrm{CH}_{2} \mathrm{Cl}_{2}$ 10:90 v/v to $\mathrm{MeOH} / \mathrm{CH}_{2} \mathrm{Cl}_{2} 20: 80 \mathrm{v} / \mathrm{v}$ to $\mathrm{MeOH} / \mathrm{CH}_{2} \mathrm{Cl}_{2} / \mathrm{HCOOH}$ 20:79:1 v/v/v). The combined fractions were concentrated and re-dissolved in $\mathrm{CH}_{2} \mathrm{Cl}_{2}(100 \mathrm{~mL})$ and then washed with $1 \mathrm{M} \mathrm{HCl}(50 \mathrm{~mL} \times 2)$. The combined aqueous layers were back-extracted with $\mathrm{CH}_{2} \mathrm{Cl}_{2}(2 \times 50 \mathrm{~mL})$. The combined organic layers were dried over anhydrous $\mathrm{Na}_{2} \mathrm{SO}_{4}$. Finally, the solvent was removed under reduced pressure and the residue dried under high vacuum to provide $\mathbf{4 b}$ as a white solid in $86 \%$ yield $(5.9 \mathrm{~g})$.

\section{Characterization data for $4 \mathrm{~b}$ :}

$\mathbf{R}_{\mathbf{f}}=0.32$ in $\mathrm{MeOH} / \mathrm{CH}_{2} \mathrm{Cl}_{2} 15: 85 \mathrm{v} / \mathrm{v}$.

$[\boldsymbol{\alpha}]_{\mathrm{D}}^{20}=-58.28\left(\mathrm{c} 0.5, \mathrm{CHCl}_{3}\right)$.

${ }^{1}$ H-NMR (600 MHz, $\left.\left(\mathrm{CD}_{3}\right)_{2} \mathrm{SO}\right): \delta=14.37$ (br s, 1H), 9.94 (s, 1H), 8.40-8.06 (comp, 3H), 7.75 (s, 1H), 5.12-4.99 (m, 1H), 4.64-4.50 (m, 1H), 2.17-2.05 (m, 1H), 2.04-1.93 (m, 1H), 1.81-1.62 (comp, 2H), 1.56-1.24 (comp, 4H).

${ }^{13} \mathrm{C}-\mathrm{NMR}\left(150 \mathrm{MHz},\left(\mathrm{CD}_{3}\right)_{2} \mathrm{SO}\right): \delta=180.2,165.5,163.9,141.8,136.4,134.3,131.7,130.8,130.0\left(\mathrm{q}, J_{C-F}=30.7\right.$ $\mathrm{Hz}), 123.2\left(\mathrm{q}, J_{C-F}=272.7 \mathrm{~Hz}\right), 122.7,122.0,121.7,116.4,76.3,55.2,30.2,29.6,23.5,23.0$.

${ }^{19}$ F-NMR $\left(565 \mathrm{MHz},\left(\mathrm{CD}_{3}\right)_{2} \mathrm{SO}\right): \delta=-61.51$.

HRMS (ESI-TOF): Calculated for $\mathrm{C}_{23} \mathrm{H}_{15}{ }^{79} \mathrm{Br}^{79} \mathrm{Br}^{79} \mathrm{Br}^{81} \mathrm{BrF}_{6} \mathrm{~N}_{2} \mathrm{O}_{4} \mathrm{~S}$ [M - H] $]^{-}$: 846.7375, found 846.7389; calculated for $\mathrm{C}_{23} \mathrm{H}_{15}{ }^{79} \mathrm{Br}^{79} \mathrm{Br}^{81} \mathrm{Br}^{81} \mathrm{BrF}_{6} \mathrm{~N}_{2} \mathrm{O}_{4} \mathrm{~S} \quad[\mathrm{M} \quad-\mathrm{H}]^{-}$: 848.7355, found 848.7370; calculated for $\mathrm{C}_{23} \mathrm{H}_{15}{ }^{79} \mathrm{Br}^{81} \mathrm{Br}^{81} \mathrm{Br}^{81} \mathrm{BrF}_{6} \mathrm{~N}_{2} \mathrm{O}_{4} \mathrm{~S}[\mathrm{M}-\mathrm{H}]^{-}: 850.7334$, found 850.7351 . 
2-((((1R,2S)-2-(3-(3,5-bis(trifluoromethyl)phenyl)thioureido)cyclohexyl)oxy)carbonyl)-3,4,5,6tetrabromobenzoic acid

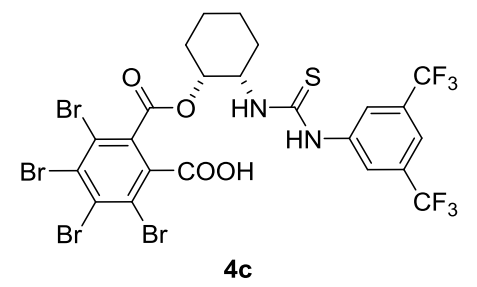

Following general procedure B, compound $4 \mathbf{c}$ was obtained from S5c (580 $\mathrm{mg}, 1.5 \mathrm{mmol}$, 1 equiv) and tetrabromophthalic anhydride ( $835 \mathrm{mg}, 1.8 \mathrm{mmol}, 1.2$ equiv) as a white solid in $65 \%$ yield $(825 \mathrm{mg})$.

\section{Characterization data for $4 \mathrm{c}$ :}

$\mathbf{R}_{\mathbf{f}}=0.34 \mathrm{in} \mathrm{MeOH} / \mathrm{CH}_{2} \mathrm{Cl}_{2} 15: 85 \mathrm{v} / \mathrm{v}$.

$[\boldsymbol{\alpha}]_{\mathbf{D}}^{\mathbf{2 0}}=+38.24\left(\mathrm{c} 0.5, \mathrm{CHCl}_{3}\right)$

${ }^{1}$ H-NMR $\left(600 \mathrm{MHz},\left(\mathrm{CD}_{3}\right)_{2} \mathrm{SO}\right): \delta=10.04(\mathrm{~s}, 1 \mathrm{H}), 8.23(\mathrm{~s}, 2 \mathrm{H}), 7.88(\mathrm{~d}, J=7.7 \mathrm{~Hz}, 1 \mathrm{H}), 7.74(\mathrm{~s}, 1 \mathrm{H}), 5.51-5.41$ (m, 1H), 4.54-4.43 (m, 1H), 2.08-1.98 (m, 1H), 1.82-1.36 (comp, 7H).

${ }^{13}$ C-NMR $\left(150 \mathrm{MHz},\left(\mathrm{CD}_{3}\right)_{2} \mathrm{SO}\right): \delta=179.8,165.9,164.1,141.7,136.7,134.5,131.6,130.7,130.1\left(\mathrm{q}, J_{C-F}=32.7\right.$ $\mathrm{Hz}), 123.2\left(\mathrm{q}, J_{C-F}=272.9 \mathrm{~Hz}\right), 122.2,122.0,121.9,116.3,73.7,53.3,27.6,26.6,23.3,19.7$.

${ }^{19}$ F-NMR (565 MHz, $\left.\left(\mathrm{CD}_{3}\right)_{2} \mathrm{SO}\right): \delta=-61.55$.

HRMS (ESI-TOF): Calculated for $\mathrm{C}_{23} \mathrm{H}_{15}{ }^{79} \mathrm{Br}^{79} \mathrm{Br}^{79} \mathrm{Br}^{81} \mathrm{BrF}_{6} \mathrm{~N}_{2} \mathrm{O}_{4} \mathrm{~S}$ [M - H] $]^{-}$: 846.7375, found 846.7379; calculated for $\mathrm{C}_{23} \mathrm{H}_{15}{ }^{79} \mathrm{Br}^{79} \mathrm{Br}^{81} \mathrm{Br}^{81} \mathrm{BrF}_{6} \mathrm{~N}_{2} \mathrm{O}_{4} \mathrm{~S} \quad[\mathrm{M} \quad-\mathrm{H}]^{-}$: 848.7355, found 848.7362; calculated for $\mathrm{C}_{23} \mathrm{H}_{15}{ }^{79} \mathrm{Br}^{81} \mathrm{Br}^{81} \mathrm{Br}^{81} \mathrm{BrF}_{6} \mathrm{~N}_{2} \mathrm{O}_{4} \mathrm{~S}[\mathrm{M}-\mathrm{H}]^{-}:$: 850.7334, found 850.7341 . 
2,3,4,5-Tetrabromo-6-((((1R,2R)-2-(3-(4-bromo-3,5-bis(trifluoromethyl)phenyl)thioureido)cyclohexyl)oxy)carbonyl)benzoic acid

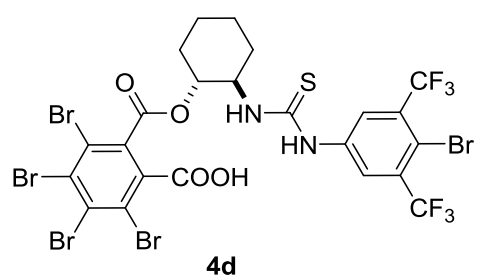

Following general procedure B, compound 4d was obtained from S5d (698 mg, $1.5 \mathrm{mmol}, 1$ equiv) and tetrabromophthalic anhydride ( $835 \mathrm{mg}, 1.8 \mathrm{mmol}, 1.2$ equiv) as a white solid in $84 \%$ yield $(1.17 \mathrm{~g})$.

\section{Characterization data for $4 \mathrm{~d}$ :}

$\mathbf{R}_{\mathbf{f}}=0.31$ in $\mathrm{MeOH} / \mathrm{CH}_{2} \mathrm{Cl}_{2} 15: 85 \mathrm{v} / \mathrm{v}$.

$[\boldsymbol{\alpha}]_{\mathbf{D}}^{20}=-48.84\left(\mathrm{c} 0.5, \mathrm{CHCl}_{3}\right)$.

${ }^{1}$ H-NMR $\left(600 \mathrm{MHz},\left(\mathrm{CD}_{3}\right)_{2} \mathrm{SO}\right): \delta=14.43(\mathrm{br} \mathrm{s}, 1 \mathrm{H}), 10.05$ (s, 1H), 8.43-8.17 (comp, 3H), 5.13-4.95 (m, 1H), 4.63-4.45 (m, 1H), 2.14-2.04 (m, 1H), 2.02-1.94 (m, 1H), 1.79-1.61 (comp, 2H), 1.53-1.18 (comp, 4H).

${ }^{13}$ C-NMR (150 MHz, $\left.\left(\mathrm{CD}_{3}\right)_{2} \mathrm{SO}\right): \delta=180.0,165.5,163.9,140.3,136.4,134.3,131.7,130.8,130.5,124.8,122.5$ (q, $\left.J_{C-F}=273.8 \mathrm{~Hz}\right), 122.1,121.7,109.8,76.3,55.2,30.1,29.5,23.4,22.9$.

${ }^{19}$ F-NMR (565 MHz, $\left.\left(\mathrm{CD}_{3}\right){ }_{2} \mathrm{SO}\right): \delta=-61.05$.

HRMS (ESI-TOF): Calculated for $\mathrm{C}_{23} \mathrm{H}_{14}{ }^{79} \mathrm{Br}^{79} \mathrm{Br}^{79} \mathrm{Br}^{81} \mathrm{Br}^{81} \mathrm{BrF}_{6} \mathrm{~N}_{2} \mathrm{O}_{4} \mathrm{~S}$ [M - H] : 926.6460, found 926.6474; calculated for $\mathrm{C}_{23} \mathrm{H}_{14}{ }^{79} \mathrm{Br}^{79} \mathrm{Br}^{81} \mathrm{Br}^{81} \mathrm{Br}^{81} \mathrm{BrF}_{6} \mathrm{~N}_{2} \mathrm{O}_{4} \mathrm{~S}[\mathrm{M}-\mathrm{H}]^{-}$: 928.6439, found 928.6457. 
2,3,4,5-Tetrabromo-6-((((1R,2R)-2-(3-(4-cyano-3,5-bis(trifluoromethyl)phenyl)thioureido)cyclohexyl)oxy)carbonyl)benzoic acid

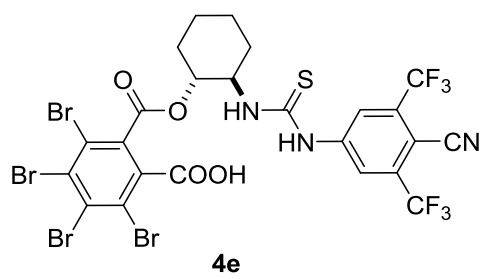

Following general procedure B, compound 4e was obtained from S5e (617 $\mathrm{mg}, 1.5 \mathrm{mmol}$, 1 equiv) and tetrabromophthalic anhydride $(835 \mathrm{mg}, 1.8 \mathrm{mmol}, 1.2$ equiv) as a white solid in $78 \%$ yield $(1.02 \mathrm{~g})$.

\section{Characterization data for $4 \mathrm{e}$ :}

$\mathbf{R}_{\mathbf{f}}=0.29 \mathrm{in} \mathrm{MeOH} / \mathrm{CH}_{2} \mathrm{Cl}_{2} 15: 85 \mathrm{v} / \mathrm{v}$.

$[\alpha]_{\mathbf{D}}^{20}=-32.68\left(\mathrm{c} 0.5, \mathrm{CHCl}_{3}\right)$.

${ }^{1}$ H-NMR $\left(600 \mathrm{MHz},\left(\mathrm{CD}_{3}\right)_{2} \mathrm{SO}\right): \delta=14.40(\mathrm{br} \mathrm{s}, 1 \mathrm{H}), 10.56(\mathrm{~s}, 1 \mathrm{H}), 8.62(\mathrm{~d}, J=8.6 \mathrm{~Hz}, 1 \mathrm{H}), 8.56(\mathrm{~s}, 2 \mathrm{H}), 5.14-$ $4.98(\mathrm{~m}, 1 \mathrm{H}), 4.62-4.49(\mathrm{~m}, 1 \mathrm{H}), 2.17-2.05(\mathrm{~m}, 1 \mathrm{H}), 2.02-1.94(\mathrm{~m}, 1 \mathrm{H}), 1.79-1.64$ (comp, 2H), 1.54-1.25 (comp, $4 \mathrm{H})$.

${ }^{13}$ C-NMR $\left(150 \mathrm{MHz},\left(\mathrm{CD}_{3}\right)_{2} \mathrm{SO}\right): \delta=179.4,165.5,163.9,145.5,136.5,134.2,133.6\left(\mathrm{q}, J_{C-F}=32.6 \mathrm{~Hz}\right), 131.8$, $130.8,122.1,121.9\left(\mathrm{q}, J_{C-F}=274.3 \mathrm{~Hz}\right), 121.7,121.0,112.6,97.5,76.2,55.2,29.9,29.5,23.4,22.9$.

${ }^{19}$ F-NMR $\left(565 \mathrm{MHz},\left(\mathrm{CD}_{3}\right)_{2} \mathrm{SO}\right): \delta=-61.18$.

HRMS (ESI-TOF): Calculated for $\mathrm{C}_{24} \mathrm{H}_{14}{ }^{79} \mathrm{Br}^{79} \mathrm{Br}^{79} \mathrm{Br}^{81} \mathrm{BrF}_{6} \mathrm{~N}_{3} \mathrm{O}_{4} \mathrm{~S}$ [M - H] : 871.7328, found: 871.7346; calculated for $\mathrm{C}_{24} \mathrm{H}_{14}{ }^{79} \mathrm{Br}^{79} \mathrm{Br}^{81} \mathrm{Br}^{81} \mathrm{BrF}_{6} \mathrm{~N}_{3} \mathrm{O}_{4} \mathrm{~S} \quad[\mathrm{M}-\mathrm{H}]^{-}$: 873.7307, found: 873.7327; calculated for $\mathrm{C}_{24} \mathrm{H}_{14}{ }^{79} \mathrm{Br}^{81} \mathrm{Br}^{81} \mathrm{Br}^{81} \mathrm{BrF}_{6} \mathrm{~N}_{3} \mathrm{O}_{4} \mathrm{~S}[\mathrm{M}-\mathrm{H}]^{-}: 875.7287$, found: 875.7308 . 
2,3,4,5-Tetrabromo-6-((((1R,2R)-2-(3-(4-nitro-3-(trifluoromethyl)phenyl)thioureido)cyclohexyl)oxy)carbonyl)benzoic acid

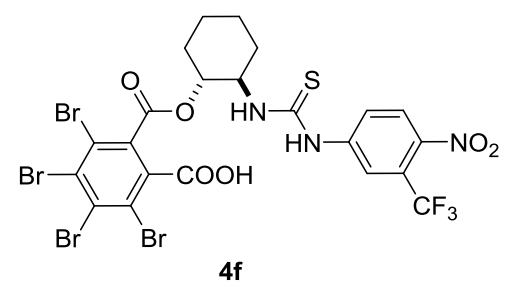

Following general procedure B, compound $4 \mathbf{f}$ was obtained from S5f (545 mg, $1.5 \mathrm{mmol}$, 1 equiv) and tetrabromophthalic anhydride ( $835 \mathrm{mg}, 1.8 \mathrm{mmol}, 1.2$ equiv) as a light-yellow solid in 67\% yield (833 $\mathrm{mg})$.

\section{Characterization data for $\mathbf{4 f :}$}

$\mathbf{R}_{\mathbf{f}}=0.29 \mathrm{in} \mathrm{MeOH} / \mathrm{CH}_{2} \mathrm{Cl}_{2} 15: 85 \mathrm{v} / \mathrm{v}$.

$[\alpha]_{\mathbf{D}}^{20}=-14.56\left(\mathrm{c} 0.5, \mathrm{CHCl}_{3}\right)$.

${ }^{1}$ H-NMR $\left(600 \mathrm{MHz},\left(\mathrm{CD}_{3}\right)_{2} \mathrm{SO}\right): \delta=14.40(\mathrm{br} \mathrm{s}, 1 \mathrm{H}), 10.22(\mathrm{~s}, 1 \mathrm{H}), 8.38(\mathrm{~s}, 1 \mathrm{H}), 8.32(\mathrm{~d}, J=8.4 \mathrm{~Hz}, 1 \mathrm{H}), 8.15(\mathrm{~d}$, $J=8.9 \mathrm{~Hz}, 1 \mathrm{H}), 7.95(\mathrm{~d}, J=8.9 \mathrm{~Hz}, 1 \mathrm{H}), 5.13-4.94(\mathrm{~m}, 1 \mathrm{H}), 4.62-4.46(\mathrm{~m}, 1 \mathrm{H}), 2.17-2.06(\mathrm{~m}, 1 \mathrm{H}), 2.04-1.96(\mathrm{~m}$, 1H), 1.79-1.63 (comp, 2H), 1.54-1.27 (comp, 4H).

${ }^{13}$ C-NMR $\left(150 \mathrm{MHz},\left(\mathrm{CD}_{3}\right)_{2} \mathrm{SO}\right): \delta=179.7,165.5,163.9,144.7,141.1,136.5,134.2,131.8,130.9,127.0,124.51$, $124.49,122.08\left(\mathrm{q}, J_{C-F}=273.1 \mathrm{~Hz}\right), 122.07,121.7,119.8,76.2,55.2,30.0,29.5,23.4,22.9$.

${ }^{19}$ F-NMR (565 MHz, $\left.\left(\mathrm{CD}_{3}\right)_{2} \mathrm{SO}\right): \delta=-58.99$.

HRMS (ESI-TOF): Calculated for $\mathrm{C}_{22} \mathrm{H}_{15}{ }^{79} \mathrm{Br}^{79} \mathrm{Br}^{79} \mathrm{Br}^{81} \mathrm{BrF}_{3} \mathrm{~N}_{3} \mathrm{O}_{6} \mathrm{~S}$ [M - H] : 823.7352, found: 823.7373; calculated for $\mathrm{C}_{22} \mathrm{H}_{15}{ }^{79} \mathrm{Br}^{79} \mathrm{Br}^{81} \mathrm{Br}^{81} \mathrm{BrF}_{3} \mathrm{~N}_{3} \mathrm{O}_{6} \mathrm{~S} \quad[\mathrm{M}-\mathrm{H}]$ : : 825.7332, found: 825.7355; calculated for $\mathrm{C}_{22} \mathrm{H}_{15}{ }^{79} \mathrm{Br}^{81} \mathrm{Br}^{81} \mathrm{Br}^{81} \mathrm{BrF}_{3} \mathrm{~N}_{3} \mathrm{O}_{6} \mathrm{~S}[\mathrm{M}-\mathrm{H}]^{-}:$: 27.7311 , found: 827.7335 . 
2,3,4,5-Tetrabromo-6-((((1R,2R)-2-(3-(3-(trifluoromethyl)phenyl)thioureido)cyclohexyl)oxy)carbonyl)benzoic acid

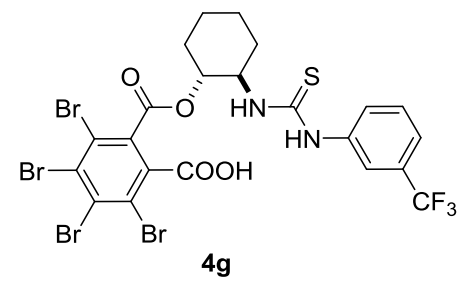

Following general procedure B, compound $4 \mathrm{~g}$ was obtained from S5g (478 $\mathrm{mg}, 1.5 \mathrm{mmol}, 1$ equiv) and tetrabromophthalic anhydride $(835 \mathrm{mg}, 1.8 \mathrm{mmol}, 1.2$ equiv) as a white solid in $88 \%$ yield $(1.03 \mathrm{~g})$.

\section{Characterization data for $4 \mathrm{~g}$ :}

$\mathbf{R}_{\mathbf{f}}=0.28$ in $\mathrm{MeOH} / \mathrm{CH}_{2} \mathrm{Cl}_{2} 15: 85 \mathrm{v} / \mathrm{v}$.

$[\boldsymbol{\alpha}]_{\mathbf{D}}^{20}=-30.68\left(\mathrm{c} 0.5, \mathrm{CHCl}_{3}\right)$.

${ }^{1}$ H-NMR $\left(600 \mathrm{MHz},\left(\mathrm{CD}_{3}\right)_{2} \mathrm{SO}\right): \delta=14.43(\mathrm{~s}, 1 \mathrm{H}), 9.71(\mathrm{~s}, 1 \mathrm{H}), 8.12-7.85$ (comp, 2H), 7.69-7.62 (m, 1H), 7.57$7.49(\mathrm{~m}, 1 \mathrm{H}), 7.46-7.40(\mathrm{~m}, 1 \mathrm{H}), 5.14-4.96(\mathrm{~m}, 1 \mathrm{H}), 4.69-4.44(\mathrm{~m}, 1 \mathrm{H}), 2.12-1.95$ (comp, 2H), 1.80-1.60 (comp, 2H), $1.53-1.23$ (comp, 4H).

${ }^{13}$ C-NMR $\left(150 \mathrm{MHz},\left(\mathrm{CD}_{3}\right)_{2} \mathrm{SO}\right): \delta=180.1,165.5,163.9,140.3,136.4,134.3,131.7,130.9,129.5,128.9\left(\mathrm{q}, J_{C-F}=\right.$ $30.8 \mathrm{~Hz}), 126.5,124.1$ (q, $\left.J_{C-F}=272.3 \mathrm{~Hz}\right), 122.0,121.7,120.2,119.2,76.3,55.0,30.3,29.6,23.5,23.0$.

${ }^{19}$ F-NMR $\left(565 \mathrm{MHz},\left(\mathrm{CD}_{3}\right)_{2} \mathrm{SO}\right): \delta=-61.18$.

HRMS (ESI-TOF): Calculated for $\mathrm{C}_{22} \mathrm{H}_{16}{ }^{79} \mathrm{Br}^{79} \mathrm{Br}^{79} \mathrm{Br}^{81} \mathrm{BrF}_{3} \mathrm{~N}_{2} \mathrm{O}_{4} \mathrm{~S}$ [M - H] : 778.7501, found: 778.7500; calculated for $\mathrm{C}_{22} \mathrm{H}_{16}{ }^{79} \mathrm{Br}^{79} \mathrm{Br}^{81} \mathrm{Br}^{81} \mathrm{BrF}_{3} \mathrm{~N}_{2} \mathrm{O}_{4} \mathrm{~S}$ [M - H] : 780.7481, found: 780.7483; calculated for $\mathrm{C}_{22} \mathrm{H}_{16}{ }^{79} \mathrm{Br}^{81} \mathrm{Br}^{81} \mathrm{Br}^{81} \mathrm{BrF}_{3} \mathrm{~N}_{2} \mathrm{O}_{4} \mathrm{~S}[\mathrm{M}-\mathrm{H}]^{-}:$: 782.7460, found: 782.7463 . 
2-(((1S,2S)-2-(3-(3,5-bis(trifluoromethyl)phenyl)thioureido)-1,2-diphenylethoxy)carbonyl)-3,4,5,6tetrabromobenzoic acid

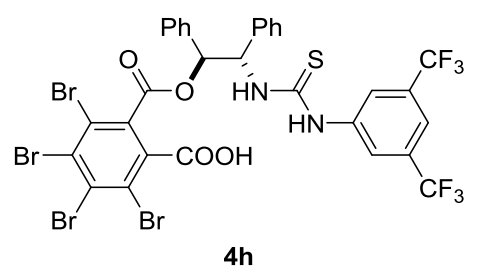

To an ice cooled solution of (1S,2S)-2-amino-1,2-diphenylethan-1-ol (213 mg, $1 \mathrm{mmol})$ in THF (10 mL) was slowly added 3,5-bis(trifluoromethyl)phenyl isothiocyanate $(0.183 \mathrm{ml}, 1 \mathrm{mmol}, 1$ equiv). The resulting mixture was stirred at the same temperature for $30 \mathrm{~min}$ and then 4- $N, N$-dimethylaminopyridine (DMAP) (147 $\mathrm{mg}, 1.2 \mathrm{mmol}, 1.2$ equiv) and tetrabromophthalic anhydride $(556 \mathrm{mg}, 1.2 \mathrm{mmol}, 1.2$ equiv) was added. The resulting mixture was warmed to room temperature and stirred for $1.5 \mathrm{~h}$ and then transferred to a separatory funnel. EtOAc $(100 \mathrm{~mL})$ was added and the solution was washed with $1 \mathrm{M} \mathrm{HCl}(50 \mathrm{~mL} \times 2)$, saturated aqueous $\mathrm{NaHCO}_{3}(50 \mathrm{~mL})$, and $1 \mathrm{M} \mathrm{HCl}(50 \mathrm{~mL})$, and dried over anhydrous $\mathrm{Na}_{2} \mathrm{SO}_{4}$. The solvent was removed under reduced pressure and the residue purified by neutral $\mathrm{Al}_{2} \mathrm{O}_{3}$ chromatography (using a gradient eluent from $\mathrm{CH}_{2} \mathrm{Cl}_{2}$ to $\mathrm{MeOH} / \mathrm{CH}_{2} \mathrm{Cl}_{2} 10: 90 \mathrm{v} / \mathrm{v}$ to $\mathrm{MeOH} / \mathrm{CH}_{2} \mathrm{Cl}_{2}$ 20:80 v/v to $\mathrm{MeOH} / \mathrm{CH}_{2} \mathrm{Cl}_{2} / \mathrm{HCOOH} 20: 79: 1 \mathrm{v} / \mathrm{v} / \mathrm{v}$ ). The combined fractions were concentrated and re-dissolved in $\mathrm{CH}_{2} \mathrm{Cl}_{2}(50 \mathrm{~mL})$ and then washed with $1 \mathrm{M} \mathrm{HCl}(25 \mathrm{~mL} \times 2)$. The combined aqueous layers were back-extracted with $\mathrm{CH}_{2} \mathrm{Cl}_{2}(2 \times 25 \mathrm{~mL})$. The combined organic layers were dried over anhydrous $\mathrm{Na}_{2} \mathrm{SO}_{4}$. Finally, the solvent was removed under reduced pressure and the resulting solid was dried under high vacuum to provide $\mathbf{4 h}$ as a white solid in $77 \%$ yield over two steps $(730 \mathrm{mg})$.

\section{Characterization data for $4 \mathrm{~h}$ :}

$\mathbf{R}_{\mathbf{f}}=0.46$ in $\mathrm{MeOH} / \mathrm{CH}_{2} \mathrm{Cl}_{2} 15: 85 \mathrm{v} / \mathrm{v}$.

$[\boldsymbol{\alpha}]_{\mathbf{D}}^{\mathbf{2 0}}=+12.06\left(\mathrm{c} 0.5, \mathrm{CHCl}_{3}\right)$

${ }^{1}$ H-NMR $\left(600 \mathrm{MHz},\left(\mathrm{CD}_{3}\right)_{2} \mathrm{SO}\right): \delta=14.26$ (br s, $\left.1 \mathrm{H}\right), 10.44$ (s, $\left.1 \mathrm{H}\right), 8.87(\mathrm{~d}, J=9.8 \mathrm{~Hz}, 1 \mathrm{H}), 8.25(\mathrm{~s}, 2 \mathrm{H}), 7.75$ (s, 1H), 7.35-7.30 (comp, 4H), 7.29-7.25 (comp, 5H), 7.24-7.20 (m, 1H), 6.42 (d, $J=6.5 \mathrm{~Hz}, 1 \mathrm{H}), 6.14$ (app t, $J=7.5$ $\mathrm{Hz}, 1 \mathrm{H})$.

${ }^{13}$ C-NMR $\left(150 \mathrm{MHz},\left(\mathrm{CD}_{3}\right)_{2} \mathrm{SO}\right): \delta=180.8,165.2,163.5,141.7,137.8,136.8,135.9,133.6,131.9,130.7,130.1(\mathrm{q}$, $\left.J_{C-F}=32.5 \mathrm{~Hz}\right), 128.22,128.16,127.9,127.8,127.5,127.3,123.2\left(\mathrm{q}, J_{C-F}=272.7 \mathrm{~Hz}\right), 122.2,122.1,121.8,116.5$, $80.3,61.0$.

${ }^{19}$ F-NMR $\left(565 \mathrm{MHz},\left(\mathrm{CD}_{3}\right){ }_{2} \mathrm{SO}\right): \delta=-61.55$.

HRMS (ESI-TOF): Calculated for $\mathrm{C}_{31} \mathrm{H}_{17}{ }^{79} \mathrm{Br}^{79} \mathrm{Br}^{79} \mathrm{Br}^{81} \mathrm{BrF}_{6} \mathrm{~N}_{2} \mathrm{O}_{4} \mathrm{~S}$ [M - H] : 944.7532, found: 944.7553; calculated for $\mathrm{C}_{31} \mathrm{H}_{17}{ }^{79} \mathrm{Br}^{79} \mathrm{Br}^{81} \mathrm{Br}^{81} \mathrm{BrF}_{6} \mathrm{~N}_{2} \mathrm{O}_{4} \mathrm{~S}$ [M $\left.-\mathrm{H}\right]^{-}$: 946.7511, found: 946.7535; calculated for $\mathrm{C}_{31} \mathrm{H}_{17}{ }^{79} \mathrm{Br}^{81} \mathrm{Br}^{81} \mathrm{Br}^{81} \mathrm{BrF}_{6} \mathrm{~N}_{2} \mathrm{O}_{4} \mathrm{~S}[\mathrm{M}-\mathrm{H}]^{-}: 948.7491$, found: 948.7518 . 
2-(((1S,2R)-2-(3-(3,5-bis(trifluoromethyl)phenyl)thioureido)-1,2-diphenylethoxy)carbonyl)-3,4,5,6tetrabromobenzoic acid

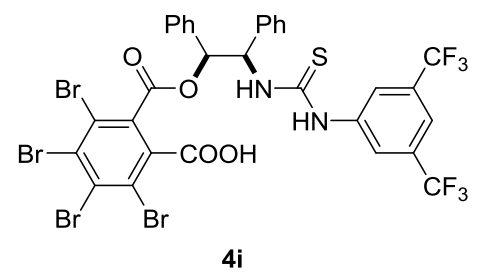

To an ice cooled solution of (1S,2R)-2-amino-1,2-diphenylethan-1-ol (427 mg, $2 \mathrm{mmol})$ in THF (20 mL) was slowly added 3,5-bis(trifluoromethyl)phenyl isothiocyanate $(0.365 \mathrm{ml}, 2 \mathrm{mmol}, 1$ equiv). The resulting mixture was stirred at the same temperature for $30 \mathrm{~min}$ and then 4- $N, N$-dimethylaminopyridine (DMAP) (293 $\mathrm{mg}, 2.4 \mathrm{mmol}, 1.2$ equiv) and tetrabromophthalic anhydride $(1.1 \mathrm{~g}, 2.4 \mathrm{mmol}, 1.2$ equiv) was added. The resulting mixture was warmed to room temperature and stirred for $1.5 \mathrm{~h}$ and then transferred to a separatory funnel. EtOAc $(100 \mathrm{~mL})$ was added and the solution was washed with $1 \mathrm{M} \mathrm{HCl}(50 \mathrm{~mL} \times 2)$, saturated aqueous $\mathrm{NaHCO}_{3}(50 \mathrm{~mL})$, and $1 \mathrm{M} \mathrm{HCl}(50 \mathrm{~mL})$, and dried over anhydrous $\mathrm{Na}_{2} \mathrm{SO}_{4}$. The solvent was removed under reduced pressure and the residue purified by neutral $\mathrm{Al}_{2} \mathrm{O}_{3}$ chromatography (using a gradient eluent from $\mathrm{CH}_{2} \mathrm{Cl}_{2}$ to $\mathrm{MeOH} / \mathrm{CH}_{2} \mathrm{Cl}_{2} 10: 90 \mathrm{v} / \mathrm{v}$ to $\mathrm{MeOH} / \mathrm{CH}_{2} \mathrm{Cl}_{2}$ 20:80 v/v to MeOH/ $\mathrm{CH}_{2} \mathrm{Cl}_{2} / \mathrm{HCOOH}$ 20:79:1 v/v/v). The combined fractions were concentrated and re-dissolved in $\mathrm{CH}_{2} \mathrm{Cl}_{2}(50 \mathrm{~mL})$ and then washed with $1 \mathrm{M} \mathrm{HCl}(25 \mathrm{~mL} \times 2)$. The combined aqueous layers were back-extracted with $\mathrm{CH}_{2} \mathrm{Cl}_{2}(2 \times 25 \mathrm{~mL})$. The combined organic layers were dried over anhydrous $\mathrm{Na}_{2} \mathrm{SO}_{4}$. Finally, the solvent was removed under reduced pressure and the resulting solid was dried under high vacuum to provide $\mathbf{4} \mathbf{i}$ as a white solid in $72 \%$ yield over two steps $(1.36 \mathrm{~g})$.

\section{Characterization data for $4 \mathrm{i}$ :}

$\mathbf{R}_{\mathbf{f}}=0.44$ in $\mathrm{MeOH} / \mathrm{CH}_{2} \mathrm{Cl}_{2}$ 15:85 v/v.

$[\boldsymbol{\alpha}]_{\mathbf{D}}^{20}=+14.90\left(\mathrm{c} 0.5, \mathrm{CHCl}_{3}\right)$.

${ }^{1}$ H-NMR (600 MHz, $\left.\left(\mathrm{CD}_{3}\right)_{2} \mathrm{SO}\right): \delta=10.35(\mathrm{~s}, 1 \mathrm{H}), 8.54(\mathrm{~d}, J=8.4 \mathrm{~Hz}, 1 \mathrm{H}), 8.17(\mathrm{~s}, 2 \mathrm{H}), 7.76(\mathrm{~s}, 1 \mathrm{H}), 7.35-7.27$ (comp, 6H), 7.26-7.23 (comp, 2H), 7.22-7.18 (comp, 2H), 6.50 (d, $J=5.3 \mathrm{~Hz}, 1 \mathrm{H}), 6.04$ (dd, $J=8.6,5.3 \mathrm{~Hz}, 1 \mathrm{H})$.

${ }^{13}$ C-NMR (150 MHz, $\left.\left(\mathrm{CD}_{3}\right)_{2} \mathrm{SO}\right): \delta=180.2,165.6,163.8,141.5,137.2,136.3,135.4,133.5,132.0,130.6,130.2(\mathrm{q}$, $\left.J_{C-F}=32.6 \mathrm{~Hz}\right), 128.5,128.24,128.18,128.03,127.96,126.8,123.2\left(\mathrm{q}, J_{C-F}=272.9 \mathrm{~Hz}\right), 122.3,122.0,121.9,116.6$, $79.3,61.4$.

${ }^{19}$ F-NMR (565 MHz, $\left.\left(\mathrm{CD}_{3}\right)_{2} \mathrm{SO}\right): \delta=-61.54$.

HRMS (ESI-TOF): Calculated for $\mathrm{C}_{31} \mathrm{H}_{17}{ }^{79} \mathrm{Br}^{79} \mathrm{Br}^{79} \mathrm{Br}^{81} \mathrm{BrF}_{6} \mathrm{~N}_{2} \mathrm{O}_{4} \mathrm{~S}$ [M - H] : 944.7532, found: 944.7555; calculated for $\mathrm{C}_{31} \mathrm{H}_{17}{ }^{79} \mathrm{Br}^{79} \mathrm{Br}^{81} \mathrm{Br}^{81} \mathrm{BrF}_{6} \mathrm{~N}_{2} \mathrm{O}_{4} \mathrm{~S}$ [M - H] : 946.7511, found: 946.7536; calculated for $\mathrm{C}_{31} \mathrm{H}_{17}{ }^{79} \mathrm{Br}^{81} \mathrm{Br}^{81} \mathrm{Br}^{81} \mathrm{BrF}_{6} \mathrm{~N}_{2} \mathrm{O}_{4} \mathrm{~S}[\mathrm{M}-\mathrm{H}]^{-}$: 948.7491, found: 948.7520 . 
(R)-2-((2-(3-(3,5-bis(trifluoromethyl)phenyl)thioureido)-2-phenylethoxy)carbonyl)-3,4,5,6-tetrabromobenzoic acid

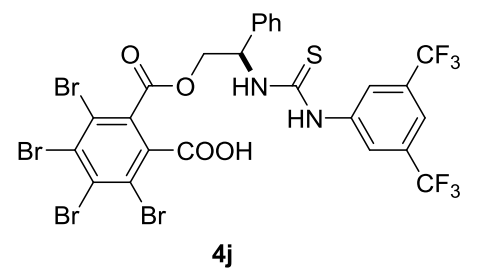

To an ice cooled solution of $(R)$-2-amino-2-phenylethan-1-ol (274 mg, $2 \mathrm{mmol})$ in THF (20 mL) was slowly added 3,5-bis(trifluoromethyl)phenyl isothiocyanate $(0.365 \mathrm{ml}, 2 \mathrm{mmol}, 1$ equiv). The resulting mixture was stirred at the same temperature for $30 \mathrm{~min}$ and then 4-N,N-dimethylaminopyridine (DMAP) (293 mg, $2.4 \mathrm{mmol}, 1.2$ equiv) and tetrabromophthalic anhydride $(1.1 \mathrm{~g}, 2.4 \mathrm{mmol}, 1.2$ equiv) was added. The resulting mixture was warmed to room temperature and stirred for $5 \mathrm{~h}$ and then transferred to a separatory funnel. EtOAc $(100 \mathrm{~mL})$ was added and the solution was washed with $1 \mathrm{M} \mathrm{HCl}(50 \mathrm{~mL} \times 2)$, saturated aqueous $\mathrm{NaHCO}_{3}(50 \mathrm{~mL})$, and $1 \mathrm{M} \mathrm{HCl}(50 \mathrm{~mL})$, and dried over anhydrous $\mathrm{Na}_{2} \mathrm{SO}_{4}$. The solvent was removed under reduced pressure and the residue purified by neutral $\mathrm{Al}_{2} \mathrm{O}_{3}$ chromatography (using a gradient eluent from $\mathrm{CH}_{2} \mathrm{Cl}_{2}$ to $\mathrm{MeOH} / \mathrm{CH}_{2} \mathrm{Cl}_{2}$ 10:90 v/v to $\mathrm{MeOH} / \mathrm{CH}_{2} \mathrm{Cl}_{2}$ 20:80 $\mathrm{v} / \mathrm{v}$ to $\mathrm{MeOH} / \mathrm{CH}_{2} \mathrm{Cl}_{2} / \mathrm{HCOOH}$ 20:79:1 v/v/v). The combined fractions were concentrated and re-dissolved in $\mathrm{CH}_{2} \mathrm{Cl}_{2}(50 \mathrm{~mL})$ and then washed with $1 \mathrm{M} \mathrm{HCl}(25 \mathrm{~mL} \times 2)$. The combined aqueous layers were back-extracted with $\mathrm{CH}_{2} \mathrm{Cl}_{2}(2 \times 25 \mathrm{~mL})$. The combined organic layers were dried over anhydrous $\mathrm{Na}_{2} \mathrm{SO}_{4}$. Finally, the solvent was removed under reduced pressure and the resulting solid was dried under high vacuum to provide $\mathbf{4} \mathbf{j}$ as a white solid in $73 \%$ yield over two steps $(1.28 \mathrm{~g})$.

\section{Characterization data for $\mathbf{4 j}$ :}

$\mathbf{R}_{\mathbf{f}}=0.38$ in $\mathrm{MeOH} / \mathrm{CH}_{2} \mathrm{Cl}_{2}$ 15:85 v/v.

$[\boldsymbol{\alpha}]_{\mathbf{D}}^{20}=+5.04\left(\mathrm{c} 0.5, \mathrm{CHCl}_{3}\right)$.

${ }^{1}$ H-NMR $\left(600 \mathrm{MHz},\left(\mathrm{CD}_{3}\right)_{2} \mathrm{SO}\right): \delta=14.32(\mathrm{br} \mathrm{s}, 1 \mathrm{H}), 10.36(\mathrm{~s}, 1 \mathrm{H}), 8.98(\mathrm{~d}, J=8.0 \mathrm{~Hz}, 1 \mathrm{H}), 8.22(\mathrm{~s}, 2 \mathrm{H}), 7.75(\mathrm{~s}$, 1H), 7.50-7.43 (comp, 2H), 7.42-7.37 (comp, 2H), 7.36-7.29 (m, 1H), 5.91 (app q, $J=6.7 \mathrm{~Hz}, 1 \mathrm{H}), 4.65$ (dd, $J=$ $11.3,7.5 \mathrm{~Hz}, 1 \mathrm{H}), 4.58(\mathrm{dd}, J=11.4,5.1 \mathrm{~Hz}, 1 \mathrm{H})$.

${ }^{13}$ C-NMR $\left(150 \mathrm{MHz},\left(\mathrm{CD}_{3}\right)_{2} \mathrm{SO}\right): \delta=\delta 180.7,165.7,164.5,141.7,138.1,136.5,134.0,132.1,131.0,130.2\left(\mathrm{q}, J_{C-F}\right.$ $=33.0 \mathrm{~Hz}), 128.6,127.8,127.3,123.2\left(\mathrm{q}, J_{C-F}=272.7 \mathrm{~Hz}\right), 122.20,122.16,122.0,116.5,67.3,56.0$.

${ }^{19}$ F-NMR (565 MHz, $\left.\left(\mathrm{CD}_{3}\right)_{2} \mathrm{SO}\right): \delta=-61.61$.

HRMS (ESI-TOF): Calculated for $\mathrm{C}_{25} \mathrm{H}_{13}{ }^{79} \mathrm{Br}^{79} \mathrm{Br}^{79} \mathrm{Br}^{81} \mathrm{BrF}_{6} \mathrm{~N}_{2} \mathrm{O}_{4} \mathrm{~S}$ [M - H] : 868.7219, found: 868.7219; calculated for $\mathrm{C}_{25} \mathrm{H}_{13}{ }^{79} \mathrm{Br}^{79} \mathrm{Br}^{81} \mathrm{Br}^{81} \mathrm{BrF}_{6} \mathrm{~N}_{2} \mathrm{O}_{4} \mathrm{~S}$ [M $\left.-\mathrm{H}\right]^{-}$: 870.7198, found: 870.7205; calculated for $\mathrm{C}_{25} \mathrm{H}_{13}{ }^{79} \mathrm{Br}^{81} \mathrm{Br}^{81} \mathrm{Br}^{81} \mathrm{BrF}_{6} \mathrm{~N}_{2} \mathrm{O}_{4} \mathrm{~S}[\mathrm{M}-\mathrm{H}]^{-}$: 872.7178, found: 872.7183 . 
2-(((1R,2S)-1-(3-(3,5-bis(trifluoromethyl)phenyl)thioureido)-2,3-dihydro-1H-inden-2-yl)oxy)carbonyl)$3,4,5,6$-tetrabromobenzoic acid

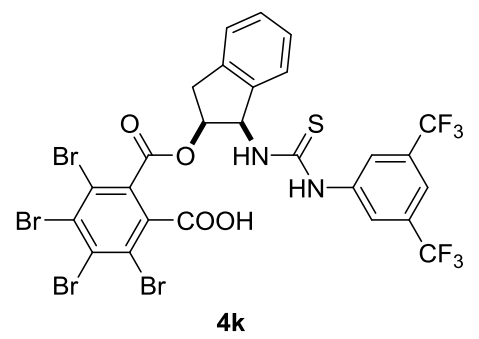

To an ice cooled solution of $(1 R, 2 S)$-1-amino-2,3-dihydro- $1 H$-inden-2-ol $(298 \mathrm{mg}, 2 \mathrm{mmol})$ in THF (20 mL) was slowly added 3,5-bis(trifluoromethyl)phenyl isothiocyanate $(0.365 \mathrm{ml}, 2 \mathrm{mmol}, 1$ equiv). The resulting mixture was stirred at the same temperature for $30 \mathrm{~min}$ and then 4- $N, N$-dimethylaminopyridine (DMAP) (293 mg, $2.4 \mathrm{mmol}, 1.2$ equiv) and tetrabromophthalic anhydride $(1.1 \mathrm{~g}, 2.4 \mathrm{mmol}, 1.2$ equiv) was added. The resulting mixture was warmed to room temperature and stirred for $1.5 \mathrm{~h}$ and then transferred to a separatory funnel. EtOAc $(100 \mathrm{~mL})$ was added and the solution was washed with $1 \mathrm{M} \mathrm{HCl}(50 \mathrm{~mL} \times 2)$, saturated aqueous $\mathrm{NaHCO}_{3}(50 \mathrm{~mL})$, and $1 \mathrm{M} \mathrm{HCl}$ $(50 \mathrm{~mL})$, and dried over anhydrous $\mathrm{Na}_{2} \mathrm{SO}_{4}$. The solvent was removed under reduced pressure and the residue purified by neutral $\mathrm{Al}_{2} \mathrm{O}_{3}$ chromatography (using a gradient eluent from $\mathrm{CH}_{2} \mathrm{Cl}_{2}$ to $\mathrm{MeOH} / \mathrm{CH}_{2} \mathrm{Cl}_{2}$ 10:90 v/v to $\mathrm{MeOH} / \mathrm{CH}_{2} \mathrm{Cl}_{2} 20: 80 \mathrm{v} / \mathrm{v}$ to $\mathrm{MeOH} / \mathrm{CH}_{2} \mathrm{Cl}_{2} / \mathrm{HCOOH}$ 20:79:1 v/v/v). The combined fractions were concentrated and re-dissolved in $\mathrm{CH}_{2} \mathrm{Cl}_{2}(50 \mathrm{~mL})$ and then washed with $1 \mathrm{M} \mathrm{HCl}(25 \mathrm{~mL} \times 2)$. The combined aqueous layers were back-extracted with $\mathrm{CH}_{2} \mathrm{Cl}_{2}(2 \times 25 \mathrm{~mL})$. The combined organic layers were dried over anhydrous $\mathrm{Na}_{2} \mathrm{SO}_{4}$. Finally, the solvent was removed under reduced pressure and the resulting solid was dried under high vacuum to provide $\mathbf{4 k}$ as a white solid in $79 \%$ yield over two steps $(1.40 \mathrm{~g})$.

\section{Characterization data for $4 \mathrm{k}$ :}

$\mathbf{R}_{\mathbf{f}}=0.43$ in $\mathrm{MeOH} / \mathrm{CH}_{2} \mathrm{Cl}_{2}$ 15:85 v/v.

$[\alpha]_{\mathbf{D}}^{20}=-3.74\left(\mathrm{c} 0.5, \mathrm{CHCl}_{3}\right)$

${ }^{1}$ H-NMR $\left(600 \mathrm{MHz},\left(\mathrm{CD}_{3}\right)_{2} \mathrm{SO}\right): \delta=10.36(\mathrm{~s}, 1 \mathrm{H}), 8.46(\mathrm{~d}, J=8.5 \mathrm{~Hz}, 1 \mathrm{H}), 8.32(\mathrm{~s}, 2 \mathrm{H}), 7.78(\mathrm{~s}, 1 \mathrm{H}), 7.36-7.24$ (comp, 4H), 6.25 (dd, $J=8.5,5.0 \mathrm{~Hz}, 1 \mathrm{H}), 5.91($ app t, $J=4.9 \mathrm{~Hz}, 1 \mathrm{H}), 3.41$ (dd, $J=17.2,4.7 \mathrm{~Hz}, 1 \mathrm{H}), 3.15(\mathrm{~d}, J=$ $17.2 \mathrm{~Hz}, 1 \mathrm{H})$.

${ }^{13}$ C-NMR (150 MHz, $\left.\left(\mathrm{CD}_{3}\right)_{2} \mathrm{SO}\right): \delta=181.5,165.7,164.0,141.7,139.7,139.6,136.7,134.1,131.6,130.5,130.2(\mathrm{q}$, $\left.J_{C-F}=32.8 \mathrm{~Hz}\right), 128.2,126.8,125.1,123.4,123.2\left(\mathrm{q}, J_{C-F}=272.7 \mathrm{~Hz}\right), 122.5,121.94,121.89,116.6,78.3,60.2$, 36.3 .

${ }^{19}$ F-NMR $\left(565 \mathrm{MHz},\left(\mathrm{CD}_{3}\right){ }_{2} \mathrm{SO}\right): \delta=-61.49$.

HRMS (ESI-TOF): Calculated for $\mathrm{C}_{26} \mathrm{H}_{13}{ }^{79} \mathrm{Br}^{79} \mathrm{Br}^{79} \mathrm{Br}^{81} \mathrm{BrF}_{6} \mathrm{~N}_{2} \mathrm{O}_{4} \mathrm{~S}$ [M - H] : 880.7219, found: 880.7226; calculated for $\mathrm{C}_{26} \mathrm{H}_{13}{ }^{79} \mathrm{Br}^{79} \mathrm{Br}^{81} \mathrm{Br}^{81} \mathrm{BrF}_{6} \mathrm{~N}_{2} \mathrm{O}_{4} \mathrm{~S}$ [M $\left.-\mathrm{H}\right]^{-}:$: 882.7198, found: 882.7205; calculated for $\mathrm{C}_{26} \mathrm{H}_{13}{ }^{79} \mathrm{Br}^{81} \mathrm{Br}^{81} \mathrm{Br}^{81} \mathrm{BrF}_{6} \mathrm{~N}_{2} \mathrm{O}_{4} \mathrm{~S}[\mathrm{M}-\mathrm{H}]^{-}:$: 884.7178, found: 884.7186. 


\section{Preparation and Characterization Data of Products:}

\section{General Procedure C for the Catalytic Enantioselective [4+2] Cycloaddition of Acetals}

To a flame dried vial was added the alcohol $(0.3 \mathrm{mmol}, 1.2$ equiv), acid catalyst $\mathbf{4 b}(21.2 \mathrm{mg}, 0.025 \mathrm{mmol}, 10$ mol\%), $5 \AA \mathrm{MS}(100 \mathrm{mg})$, and dry toluene $(5 \mathrm{~mL}, 0.05 \mathrm{M})$. The resulting mixture was stirred under nitrogen and cooled to the indicated temperature over 15 minutes. Acetal $(0.25 \mathrm{mmol}, 1$ equiv) was then added and the reaction mixture was stirred for the indicated time before being quenched with triethylamine $(0.1 \mathrm{~mL})$. The resulting mixture was directly purified by flash chromatography on silica gel topped with Celite using EtOAc/hexanes/Et $3 \mathrm{~N}(2 / 98 / 0.1)$ as the eluent.

Racemic products (for SFC assays) were prepared from the alcohol ( $0.12 \mathrm{mmol}, 1.2$ equiv), aldehyde ( $0.1 \mathrm{mmol}, 1$ equiv), trimethyl orthoformate ( $13 \mu \mathrm{L}, 0.12 \mathrm{mmol}, 1.2$ equiv) and toluene-4-sulfonic acid monohydrate ( $3.8 \mathrm{mg}$, $0.02 \mathrm{mmol}, 20 \mathrm{~mol} \%)$ in dry toluene $(2 \mathrm{~mL}, 0.05 \mathrm{M})$. The resulting mixture was stirred at room temperature until aldehyde was consumed as indicated by TLC and then directly purified by flash chromatography on silica gel using EtOAc/hexanes/Et ${ }_{3} \mathrm{~N}(2 / 98 / 0.1)$ as the eluent. 
(4aS,10bS)-5,5-Dimethyl-3,4,4a,10b-tetrahydro-2H,5H-pyrano[3,2-c]chromene

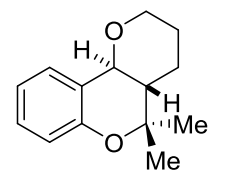

$3 \mathbf{a}$

To a flame dried vial was added 5-methylhex-4-en-1-ol (34 mg, $0.3 \mathrm{mmol}, 1.2$ equiv), acid catalyst $4 \mathrm{e}$ (10.9 mg, $0.0125 \mathrm{mmol}, 5 \mathrm{~mol} \%), 5 \AA \mathrm{MS}(100 \mathrm{mg})$, and dry toluene $(5 \mathrm{~mL}, 0.05 \mathrm{M})$. The resulting mixture was stirred under nitrogen and cooled to $0{ }^{\circ} \mathrm{C}$ over 15 minutes. Salicylaldehyde dimethyl acetal ( $\left.42 \mathrm{mg}, 0.25 \mathrm{mmol}\right)$ was then added and the reaction mixture was stirred for another $30 \mathrm{~min}$, then quenched with triethylamine $(0.1 \mathrm{~mL})$. The resulting mixture was directly purified by flash chromatography on silica gel topped with Celite using EtOAc/hexanes/Et ${ }_{3} \mathrm{~N}$ (2/98/0.1) as the eluent. Compound 3a was obtained as a white solid in $89 \%$ yield ( $49 \mathrm{mg}$ ).

\section{Characterization data for $3 a$ :}

$\mathbf{R}_{\mathbf{f}}=0.37$ in hexanes/EtOAc $90: 10 \mathrm{v} / \mathrm{v}$.

$[\boldsymbol{\alpha}]_{\mathbf{D}}^{\mathbf{2 0}}=-43.08\left(\mathrm{c} 0.5, \mathrm{CHCl}_{3}, 99 \%\right.$ ee $)$.

${ }^{1}$ H-NMR $\left(400 \mathrm{MHz}, \mathrm{CDCl}_{3}\right): \delta=7.41(\mathrm{ddd}, J=7.7,1.8,1.1 \mathrm{~Hz}, 1 \mathrm{H}), 7.15(\mathrm{dddd}, J=8.1,7.3,1.7,0.8 \mathrm{~Hz}, 1 \mathrm{H})$, 6.90 (app td, $J=7.5,1.2 \mathrm{~Hz}, 1 \mathrm{H}), 6.77$ (dd, $J=8.2,1.2 \mathrm{~Hz}, 1 \mathrm{H}), 4.24-4.12$ (comp, 2H), 3.65 (app td, $J=11.4,3.6$ $\mathrm{Hz}, 1 \mathrm{H}), 1.99-1.89$ (m, 1H), 1.86-1.67 (comp, 3H), 1.40 (s, 3H), 1.39-1.28 (m, 1H), 1.20 (s, 3H).

${ }^{13}$ C-NMR $\left(100 \mathrm{MHz}, \mathrm{CDCl}_{3}\right): \delta=152.9,128.9,126.1,122.7,120.0,116.8,78.5,73.7,68.4,45.2,27.7,26.4,25.2$, 20.6 .

HRMS (ESI-TOF): Calculated for $\mathrm{C}_{14} \mathrm{H}_{19} \mathrm{O}_{2}[\mathrm{M}+\mathrm{H}]^{+}: 219.1380$, found 219.1353 .

SFC: Daicel Chiralcel OJ-H, column temperature $=40^{\circ} \mathrm{C}, \mathrm{CO}_{2} / \mathrm{MeOH}=95 / 5$, Flow rate $=2 \mathrm{~mL} / \mathrm{min}, \mathrm{UV}=280 \mathrm{~nm}$, $t_{R}=3.7$ min (major) and $t_{R}=5.6 \min$ (minor).

The absolute configuration was assigned by analogy. 


\section{(3aS,9bS)-4,4-Dimethyl-2,3,3a,9b-tetrahydro-4H-furo[3,2-c]chromene}

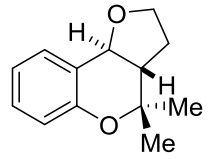

$3 \mathbf{b}$

Following general procedure $\mathrm{C}$ (reaction performed at $0{ }^{\circ} \mathrm{C}$ for $20.5 \mathrm{~h}$ ), compound $\mathbf{3 b}$ was obtained from salicylaldehyde dimethyl acetal (42 mg, $0.25 \mathrm{mmol}, 1$ equiv) and 4-methylpent-3-en-1-ol (30 mg, $0.3 \mathrm{mmol}, 1.2$ equiv) as a colorless oil in $70 \%$ yield $(36 \mathrm{mg})$.

\section{Characterization data for $3 \mathrm{~b}$ :}

$\mathbf{R}_{\mathbf{f}}=0.24$ in hexanes/EtOAc $90: 10 \mathrm{v} / \mathrm{v}$.

$[\boldsymbol{\alpha}]_{\mathbf{D}}^{20}=-1.96\left(\mathrm{c} 0.5, \mathrm{CHCl}_{3}, 96 \%\right.$ ee $)$.

${ }^{1}$ H-NMR $\left(500 \mathrm{MHz}, \mathrm{CDCl}_{3}\right): \delta=7.30(\mathrm{app} \mathrm{dt}, J=7.5,1.4 \mathrm{~Hz}, 1 \mathrm{H}), 7.16$ (dddd, $\left.J=8.2,7.4,1.7,0.8 \mathrm{~Hz}, 1 \mathrm{H}\right), 6.89$ (app td, $J=7.4,1.1 \mathrm{~Hz}, 1 \mathrm{H}), 6.82(\mathrm{dd}, J=8.2,1.1 \mathrm{~Hz}, 1 \mathrm{H}), 4.43(\mathrm{~d}, J=10.8 \mathrm{~Hz}, 1 \mathrm{H}), 4.25(\mathrm{ddd}, J=9.8,8.4,2.6$ $\mathrm{Hz}, 1 \mathrm{H}), 4.18$ (app td, $J=8.5,7.2 \mathrm{~Hz}, 1 \mathrm{H}), 2.10-1.98$ (comp, 2H), 1.90-1.79 (m, 1H), 1.49 (s, 3H), 1.32 (s, 3H).

${ }^{13}$ C-NMR (125 MHz, $\left.\mathrm{CDCl}_{3}\right): \delta=153.1,128.7,124.6,124.5,119.9,116.5,79.7,74.7,69.1,51.1,29.8,26.9,21.8$.

HRMS (ESI-TOF): Calculated for $\mathrm{C}_{13} \mathrm{H}_{17} \mathrm{O}_{2}[\mathrm{M}+\mathrm{H}]^{+}: 205.1223$, found 205.1222.

SFC: Daicel Chiralcel AD-H, column temperature $=40{ }^{\circ} \mathrm{C}, \mathrm{CO}_{2} / \mathrm{MeOH}=90 / 10$, Flow rate $=2 \mathrm{~mL} / \mathrm{min}, \mathrm{UV}=230$ $\mathrm{nm}, \mathrm{t}_{\mathrm{R}}=3.2 \min$ (minor) and $\mathrm{t}_{\mathrm{R}}=3.5 \min$ (major).

The absolute configuration was assigned by analogy. 


\section{(4bS,10bS)-11,11-Dimethyl-4b,10b-dihydro-6H,11H-isochromeno[4,3-c]chromene}

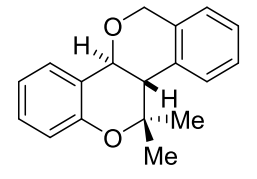

$3 \mathrm{c}$

Following general procedure $\mathrm{C}$ (reaction performed at $0{ }^{\circ} \mathrm{C}$ for $2 \mathrm{~h}$ ), compound $3 \mathbf{c}$ was obtained from salicylaldehyde dimethyl acetal (42 mg, $0.25 \mathrm{mmol}, 1$ equiv) and (2-(2-methylprop-1-en-1-yl)phenyl)methanol (49 $\mathrm{mg}, 0.3 \mathrm{mmol}, 1.2$ equiv) as a white solid in $83 \%$ yield $(55 \mathrm{mg})$.

\section{Characterization data for $3 \mathrm{c}$ :}

$\mathbf{R}_{\mathbf{f}}=0.43$ in hexanes/EtOAc $90: 10 \mathrm{v} / \mathrm{v}$.

$[\boldsymbol{\alpha}]_{\mathbf{D}}^{20}=-53.8\left(\mathrm{c} 0.5, \mathrm{CHCl}_{3}, 93 \%\right.$ ee $)$.

${ }^{1}$ H-NMR (600 MHz, $\left.\mathrm{CDCl}_{3}\right): \delta=7.61-7.57$ (m, 1H), 7.53 (app dt, $\left.J=7.7,1.3 \mathrm{~Hz}, 1 \mathrm{H}\right), 7.32-7.19$ (comp, 3H), 7.14-7.11 (m, 1H), $6.99($ app td, $J=7.5,1.2 \mathrm{~Hz}, 1 \mathrm{H}), 6.86(\mathrm{dd}, J=8.2,1.2 \mathrm{~Hz}, 1 \mathrm{H}), 5.15(\mathrm{~d}, J=14.7 \mathrm{~Hz}, 1 \mathrm{H}), 5.05$ $(\mathrm{d}, J=14.8 \mathrm{~Hz}, 1 \mathrm{H}), 4.65$ (d, $J=10.6 \mathrm{~Hz}, 1 \mathrm{H}), 3.19$ (d, $J=10.6 \mathrm{~Hz}, 1 \mathrm{H}), 1.95(\mathrm{~s}, 3 \mathrm{H}), 1.31(\mathrm{~s}, 3 \mathrm{H})$.

${ }^{13}$ C-NMR $\left(150 \mathrm{MHz}, \mathrm{CDCl}_{3}\right): \delta=152.5,136.3,134.2,129.0,126.8,126.7,125.5,125.3,123.8,120.4,116.6,80.2$, 72.0, 69.4, 47.3, 32.6, 22.3 .

HRMS (ESI-TOF): Calculated for $\mathrm{C}_{18} \mathrm{H}_{19} \mathrm{O}_{2}[\mathrm{M}+\mathrm{H}]^{+}: 267.1380$, found 267.1361.

SFC: Daicel Chiralcel AY-H, column temperature $=40{ }^{\circ} \mathrm{C}, \mathrm{CO}_{2} / \mathrm{MeOH}=90 / 10$, Flow rate $=2 \mathrm{~mL} / \mathrm{min}, \mathrm{UV}=280$ $\mathrm{nm}, \mathrm{t}_{\mathrm{R}}=4.4 \min$ (major) and $\mathrm{t}_{\mathrm{R}}=4.8 \mathrm{~min}$ (minor).

The absolute configuration was assigned by analogy. 
(4aS,10bS)-5,5-Diethyl-3,4,4a,10b-tetrahydro-2H,5H-pyrano[3,2-c]chromene

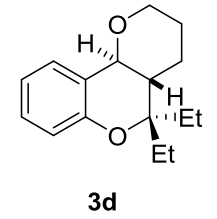

Following general procedure $\mathrm{C}$ (reaction performed at $\mathrm{rt}$ for $2.5 \mathrm{~h}$ ), compound $\mathbf{3 d}$ was obtained from salicylaldehyde dimethyl acetal (42 mg, $0.25 \mathrm{mmol}, 1$ equiv) and 5-ethylhept-4-en-1-ol (43 mg, $0.3 \mathrm{mmol}, 1.2$ equiv) as a white solid in $96 \%$ yield $(59 \mathrm{mg})$.

\section{Characterization data for $3 \mathrm{~d}$ :}

$\mathbf{R}_{\mathbf{f}}=0.38$ in hexanes/EtOAc $90: 10 \mathrm{v} / \mathrm{v}$.

$[\alpha]_{\mathrm{D}}^{20}=-45.08\left(\mathrm{c} 0.5, \mathrm{CHCl}_{3}, 93 \%\right.$ ee $)$

${ }^{1}$ H-NMR $\left(600 \mathrm{MHz} \mathrm{CDCl}_{3}\right): \delta=7.40$ (app dt, $\left.J=7.8,1.3 \mathrm{~Hz}, 1 \mathrm{H}\right), 7.17-7.12(\mathrm{~m}, 1 \mathrm{H}), 6.88(\operatorname{app~td}, J=7.5,1.2$ $\mathrm{Hz}, 1 \mathrm{H}), 6.80(\mathrm{dd}, J=8.2,1.2 \mathrm{~Hz}, 1 \mathrm{H}), 4.31$ (d, $J=10.4 \mathrm{~Hz}, 1 \mathrm{H}), 4.18-4.12(\mathrm{~m}, 1 \mathrm{H}), 3.65(\operatorname{app} \mathrm{td}, J=11.6,2.9 \mathrm{~Hz}$, 1H), 2.01-1.91 (comp, 2H), 1.86-1.79 (m, 1H), 1.79-1.67 (comp, 3H), 1.52-1.34 (comp, 3H), 0.94 (t, J = 7.4 Hz, $3 \mathrm{H}), 0.90(\mathrm{t}, J=7.4 \mathrm{~Hz}, 3 \mathrm{H})$.

${ }^{13}$ C-NMR $\left(150 \mathrm{MHz}, \mathrm{CDCl}_{3}\right): \delta=153.5,128.9,126.3,122.7,119.8,117.0,81.5,73.7,68.5,40.9,27.2,26.6,25.5$, 24.7, 7.3, 7.1.

HRMS (ESI-TOF): Calculated for $\mathrm{C}_{16} \mathrm{H}_{23} \mathrm{O}_{2}[\mathrm{M}+\mathrm{H}]^{+}:$247.1693, found 247.1687.

SFC: Daicel Chiralcel AD-H, column temperature $=40^{\circ} \mathrm{C}, \mathrm{CO}_{2} / \mathrm{MeOH}=80 / 20$, Flow rate $=2 \mathrm{~mL} / \mathrm{min}, \mathrm{UV}=280$ $\mathrm{nm}, \mathrm{t}_{\mathrm{R}}=2.6 \mathrm{~min}$ (minor) and $\mathrm{t}_{\mathrm{R}}=2.9 \min$ (major).

The absolute configuration was assigned by analogy. 
(4a'S,10b'S)-3',4',4a',10b'-Tetrahydro-2' $H$-spiro[cyclohexane-1,5'-pyrano[3,2-c]chromene]

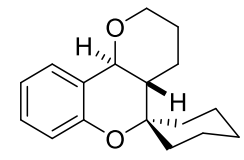

3e

Following general procedure $\mathrm{C}$ (reaction performed at $-30{ }^{\circ} \mathrm{C}$ for $12 \mathrm{~h}$ ), compound $3 \mathrm{e}$ was obtained from salicylaldehyde dimethyl acetal (42 mg, $0.25 \mathrm{mmol}, 1$ equiv) and 4-cyclohexylidenebutan-1-ol (46 mg, $0.3 \mathrm{mmol}$, 1.2 equiv) as a white solid in $74 \%$ yield $(48 \mathrm{mg})$.

\section{Characterization data for $3 \mathrm{e}$ :}

$\mathbf{R}_{\mathbf{f}}=0.37$ in hexanes/EtOAc $90: 10 \mathrm{v} / \mathrm{v}$.

$[\boldsymbol{\alpha}]_{\mathbf{D}}^{\mathbf{2 0}}=-41.92\left(\mathrm{c} 0.5, \mathrm{CHCl}_{3}, 88 \%\right.$ ee $)$.

${ }^{1}$ H-NMR $\left(400 \mathrm{MHz}, \mathrm{CDCl}_{3}\right.$ ): $\delta=7.40$ (app dt, $\left.J=7.7,1.3 \mathrm{~Hz}, 1 \mathrm{H}\right), 7.16$ (dddd, $\left.J=8.2,7.3,1.8,0.7 \mathrm{~Hz}, 1 \mathrm{H}\right), 6.89$ (app td, $J=7.5,1.2 \mathrm{~Hz}, 1 \mathrm{H}), 6.83(\mathrm{dd}, J=8.2,1.2 \mathrm{~Hz}, 1 \mathrm{H}), 4.24$ (d, $J=10.5 \mathrm{~Hz}, 1 \mathrm{H}), 4.14$ (app ddt, $J=11.4,4.1$, $1.9 \mathrm{~Hz}, 1 \mathrm{H}), 3.68-3.58$ (m, 1H), 2.02-1.88 (comp, 2H), 1.79-1.69 (comp, 4H), 1.69-1.56 (comp, 5H), 1.50-1.42 (m, 1H), 1.42-1.27 (comp, 2H), 1.24-1.11 (m, 1H).

${ }^{13}$ C-NMR $\left(100 \mathrm{MHz}, \mathrm{CDCl}_{3}\right): \delta=152.7,128.9,126.3,123.0,120.0,116.9,78.7,73.5,68.5,45.6,34.9,27.8,26.6$, 26.0, 25.0, 21.2, 21.1.

HRMS (ESI-TOF): Calculated for $\mathrm{C}_{17} \mathrm{H}_{23} \mathrm{O}_{2}[\mathrm{M}+\mathrm{H}]^{+}:$: 259.1693, found 259.1709.

SFC: Daicel Chiralcel OJ-H, column temperature $=40{ }^{\circ} \mathrm{C}, \mathrm{CO}_{2} / \mathrm{MeOH}=90 / 10$, Flow rate $=2 \mathrm{~mL} / \mathrm{min}, \mathrm{UV}=280$ $\mathrm{nm}, \mathrm{t}_{\mathrm{R}}=3.3 \mathrm{~min}$ (major) and $\mathrm{t}_{\mathrm{R}}=3.6 \mathrm{~min}$ (minor).

The absolute configuration was assigned by analogy. 
(4aS,10bS)-5,5-Dimethyl-3,4,4a,10b-tetrahydro-2H,5H-pyrano[3,2-c]chromene

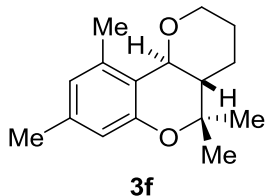

$3 f$

Following general procedure $\mathrm{C}$ (reaction performed at $-30{ }^{\circ} \mathrm{C}$ for $12 \mathrm{~h}$ ), compound $\mathbf{3 f}$ was obtained from 2(dimethoxymethyl)-3,5-dimethylphenol (49 mg, $0.25 \mathrm{mmol}, 1$ equiv) and 5-methylhex-4-en-1-ol (34 mg, $0.3 \mathrm{mmol}$, 1.2 equiv) as a white solid in $77 \%$ yield $(47 \mathrm{mg})$.

\section{Characterization data for $3 f$ :}

$\mathbf{R}_{\mathbf{f}}=0.35$ in hexanes/EtOAc $90: 10 \mathrm{v} / \mathrm{v}$.

$[\alpha]_{\mathbf{D}}^{\mathbf{2 0}}=-55.22\left(\mathrm{c} 0.5, \mathrm{CHCl}_{3}, 93 \%\right.$ ee $)$.

${ }^{1}$ H-NMR $\left(400 \mathrm{MHz}, \mathrm{CDCl}_{3}\right): \delta 6.55-6.51(\mathrm{~m}, 1 \mathrm{H}), 6.48-6.43(\mathrm{~m}, 1 \mathrm{H}), 4.17(\mathrm{~d}, J=9.8 \mathrm{~Hz}, 1 \mathrm{H}), 4.08$ (app ddt, $J=$ 11.5, 5.0, 1.6 Hz, 1H), 3.62 (app td, $J=11.7,3.2 \mathrm{~Hz}, 1 \mathrm{H}), 2.33$ (s, 3H), 2.22 (s, 3H), 1.99-1.91 (m, 1H), 1.90-1.68 (comp, 3H), 1.43-1.25 (comp, 4H), 1.12 (s, 3H).

${ }^{13}$ C-NMR $\left(100 \mathrm{MHz}, \mathrm{CDCl}_{3}\right): \delta=153.7,138.8,138.7,123.9,117.7,115.3,77.7,75.2,68.1,46.7,27.5,26.6,25.8$, $21.2,20.9,19.4$.

HRMS (ESI-TOF): Calculated for $\mathrm{C}_{16} \mathrm{H}_{22} \mathrm{KO}_{2}[\mathrm{M}+\mathrm{K}]^{+}: 285.1251$, found 285.1311.

SFC: Daicel Chiralcel OJ-H, column temperature $=40{ }^{\circ} \mathrm{C}, \mathrm{CO}_{2} / \mathrm{MeOH}=90 / 10$, Flow rate $=2 \mathrm{~mL} / \mathrm{min}, \mathrm{UV}=230$ $\mathrm{nm}, \mathrm{t}_{\mathrm{R}}=2.7 \mathrm{~min}$ (minor) and $\mathrm{t}_{\mathrm{R}}=3.6 \mathrm{~min}$ (major).

The absolute configuration was assigned by analogy. 
(4aS,10bS)-5,5-Dimethyl-3,4,4a,10b-tetrahydro-2H,5H-pyrano[3,2-c]chromene

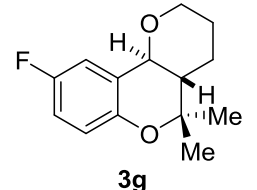

$3 \mathrm{~g}$

Following general procedure $\mathrm{C}$ (reaction performed at $0{ }^{\circ} \mathrm{C}$ for $4 \mathrm{~h}$ ), compound $\mathbf{3 g}$ was obtained from 5fluorosalicylaldehyde dimethyl acetal (47 mg, $0.25 \mathrm{mmol}, 1$ equiv) and 5-methylhex-4-en-1-ol (34 mg, $0.3 \mathrm{mmol}$, 1.2 equiv) as a white solid in $90 \%$ yield $(53 \mathrm{mg})$.

\section{Characterization data for 3g:}

$\mathbf{R}_{\mathbf{f}}=0.33$ in hexanes/EtOAc $90: 10 \mathrm{v} / \mathrm{v}$.

$[\alpha]_{\mathbf{D}}^{\mathbf{2 0}}=-56.26\left(\mathrm{c} 0.5, \mathrm{CHCl}_{3}, 99 \%\right.$ ee $)$.

${ }^{1}$ H-NMR $\left(600 \mathrm{MHz}, \mathrm{CDCl}_{3}\right): \delta=7.11(\mathrm{ddd}, J=9.2,3.2,1.0 \mathrm{~Hz}, 1 \mathrm{H}), 6.87-6.81(\mathrm{~m}, 1 \mathrm{H}), 6.70(\mathrm{dd}, J=9.0,4.7 \mathrm{~Hz}$, 1H), 4.18-4.12 (comp, 2H), 3.63 (app td, $J=11.5,3.5 \mathrm{~Hz}, 1 \mathrm{H}$ ), 1.93 (app dtd, $J=12.4,4.4,2.4 \mathrm{~Hz}, 1 \mathrm{H}), 1.82-1.71$ (comp, 2H), 1.68 (ddd, $J=12.1,10.6,3.3 \mathrm{~Hz}, 1 \mathrm{H}), 1.38$ (s, 3H), 1.32 (app qd, $J=12.3,5.0 \mathrm{~Hz}, 1 \mathrm{H}), 1.18$ (s, 3H).

${ }^{13}$ C-NMR $\left(150 \mathrm{MHz}, \mathrm{CDCl}_{3}\right): \delta=157.0\left(\mathrm{~d}, J_{C-F}=237.5 \mathrm{~Hz}\right), 148.9\left(\mathrm{~d}, J_{C-F}=2.0 \mathrm{~Hz}\right), 123.6\left(\mathrm{~d}, J_{C-F}=6.9 \mathrm{~Hz}\right)$, $117.7\left(\mathrm{~d}, J_{C-F}=7.8 \mathrm{~Hz}\right), 115.7\left(\mathrm{~d}, J_{C-F}=23.4 \mathrm{~Hz}\right), 112.3\left(\mathrm{~d}, J_{C-F}=23.6 \mathrm{~Hz}\right), 78.6,73.5,68.5,45.0,27.7,26.3,25.1$, 20.4 .

${ }^{19}$ F-NMR (565 MHz, $\left.\mathrm{CDCl}_{3}\right): \delta=-124.62$.

HRMS (ESI-TOF): Calculated for $\mathrm{C}_{14} \mathrm{H}_{18} \mathrm{FO}_{2}[\mathrm{M}+\mathrm{H}]^{+}:$: 237.1285, found 237.1294.

SFC: Daicel Chiralcel OJ-H, column temperature $=40^{\circ} \mathrm{C}, \mathrm{CO}_{2} / \mathrm{MeOH}=95 / 5$, Flow rate $=2 \mathrm{~mL} / \mathrm{min}, \mathrm{UV}=280 \mathrm{~nm}$, $t_{R}=2.6 \min$ (major) and $t_{R}=3.3 \min$ (minor).

The absolute configuration was assigned by analogy. 
(4aS,10bS)-5,5-Dimethyl-3,4,4a,10b-tetrahydro-2H,5H-pyrano[3,2-c]chromene

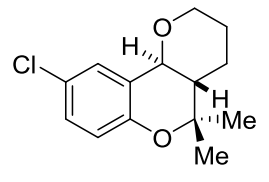

$3 h$

Following general procedure $\mathrm{C}$ (reaction performed at $0{ }^{\circ} \mathrm{C}$ for $2 \mathrm{~h}$ ), compound $\mathbf{3 h}$ was obtained from 5chlorosalicylaldehyde dimethyl acetal (51 mg, $0.25 \mathrm{mmol}, 1$ equiv) and 5-methylhex-4-en-1-ol (34 mg, $0.3 \mathrm{mmol}$, 1.2 equiv) as a white solid in $84 \%$ yield $(53 \mathrm{mg})$.

\section{Characterization data for $3 \mathrm{~h}$ :}

$\mathbf{R}_{\mathbf{f}}=0.37$ in hexanes/EtOAc $90: 10 \mathrm{v} / \mathrm{v}$.

$[\alpha]_{\mathbf{D}}^{20}=-43.18\left(\right.$ c $0.5, \mathrm{CHCl}_{3}, 96 \%$ ee $)$.

${ }^{1}$ H-NMR $\left(400 \mathrm{MHz}, \mathrm{CDCl}_{3}\right): \delta=7.39(\mathrm{dd}, J=2.8,1.1 \mathrm{~Hz}, 1 \mathrm{H}), 7.09$ (ddd, $\left.J=8.7,2.7,0.8 \mathrm{~Hz}, 1 \mathrm{H}\right), 6.69(\mathrm{~d}, J=8.7$ $\mathrm{Hz}, 1 \mathrm{H}), 4.19-4.09$ (comp, 2H), 3.68-3.57 (m, 1H), 1.96-1.88 (m, 1H), 1.82-1.62 (comp, 3H), 1.38 (s, 3H), 1.38$1.23(\mathrm{~m}, 1 \mathrm{H}), 1.17(\mathrm{~s}, 3 \mathrm{H})$.

${ }^{13}$ C-NMR $\left(100 \mathrm{MHz}, \mathrm{CDCl}_{3}\right): \delta=151.5,128.9,126.0,125.0,124.1,118.2,78.9,73.2,68.4,44.9,27.6,26.3,25.1$, 20.5.

HRMS (ESI-TOF): Calculated for $\mathrm{C}_{14} \mathrm{H}_{18}{ }^{35} \mathrm{ClO}_{2}[\mathrm{M}+\mathrm{H}]^{+}: 253.0990$, found 253.0996.

SFC: Daicel Chiralcel OJ-H, column temperature $=40^{\circ} \mathrm{C}, \mathrm{CO}_{2} / \mathrm{MeOH}=90 / 10$, Flow rate $=2 \mathrm{~mL} / \mathrm{min}$, UV $=280$ $\mathrm{nm}, \mathrm{t}_{\mathrm{R}}=2.9 \min$ (major) and $\mathrm{t}_{\mathrm{R}}=3.4 \mathrm{~min}$ (minor).

The absolute configuration was assigned by analogy. 
(4aS,10bS)-5,5-Dimethyl-3,4,4a,10b-tetrahydro-2H,5H-pyrano[3,2-c]chromene

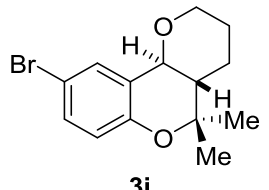

$3 \mathbf{i}$

Following general procedure $\mathrm{C}$ (reaction performed at $0{ }^{\circ} \mathrm{C}$ for $2 \mathrm{~h}$ ), compound 3i was obtained from 5bromosalicylaldehyde dimethyl acetal $(62 \mathrm{mg}, 0.25 \mathrm{mmol}, 1$ equiv) and 5-methylhex-4-en-1-ol (34 mg, $0.3 \mathrm{mmol}$, 1.2 equiv) as a white solid in $85 \%$ yield $(63 \mathrm{mg})$.

\section{Characterization data for $3 i$ :}

$\mathbf{R}_{\mathbf{f}}=0.44$ in hexanes/EtOAc $90: 10 \mathrm{v} / \mathrm{v}$.

$[\alpha]_{\mathbf{D}}^{\mathbf{2 0}}=-41.24\left(\mathrm{c} 0.5, \mathrm{CHCl}_{3}, 96 \%\right.$ ee $)$.

${ }^{1}$ H-NMR $\left(400 \mathrm{MHz}, \mathrm{CDCl}_{3}\right): \delta=7.53(\mathrm{dd}, J=2.6,1.0 \mathrm{~Hz}, 1 \mathrm{H}), 7.23(\mathrm{ddd}, J=8.8,2.6,0.8 \mathrm{~Hz}, 1 \mathrm{H}), 6.64(\mathrm{~d}, J=8.7$ $\mathrm{Hz}, 1 \mathrm{H}), 4.19-4.10$ (comp, 2H), 3.67-3.57 (m, 1H), 1.98-1.87 (m, 1H), 1.80-1.71 (comp, 2H), 1.67 (ddd, $J=12.2$, $10.6,3.3 \mathrm{~Hz}, 1 \mathrm{H}), 1.38(\mathrm{~s}, 3 \mathrm{H}) 1.38-1.26(\mathrm{~m}, 1 \mathrm{H}), 1.17(\mathrm{~s}, 3 \mathrm{H})$.

${ }^{13}$ C-NMR $\left(100 \mathrm{MHz}, \mathrm{CDCl}_{3}\right): \delta=152.0,131.8,129.0,124.6,118.7,112.3,79.0,73.2,68.4,44.9,27.6,26.3,25.1$, 20.5.

HRMS (ESI-TOF): Calculated for $\mathrm{C}_{14} \mathrm{H}_{18}{ }^{79} \mathrm{BrO}_{2}[\mathrm{M}+\mathrm{H}]^{+}:$: 297.0485, found 297.0491.

SFC: Daicel Chiralcel OJ-H, column temperature $=40{ }^{\circ} \mathrm{C}, \mathrm{CO}_{2} / \mathrm{MeOH}=90 / 10$, Flow rate $=2 \mathrm{~mL} / \mathrm{min}, \mathrm{UV}=230$ $\mathrm{nm}, \mathrm{t}_{\mathrm{R}}=3.4 \mathrm{~min}$ (major) and $\mathrm{t}_{\mathrm{R}}=3.8 \mathrm{~min}$ (minor).

The absolute configuration was assigned by analogy. 
(4aS,10bS)-5,5-Dimethyl-3,4,4a,10b-tetrahydro-2H,5H-pyrano[3,2-c]chromene

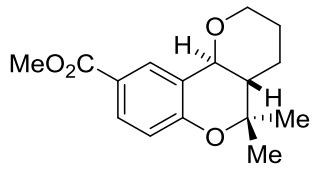

$3 \mathbf{j}$

Following general procedure $\mathrm{C}$ (reaction performed at $\mathrm{rt}$ for $2 \mathrm{~h}$ ), compound $\mathbf{3 j}$ was obtained from methyl 3(dimethoxymethyl)-4-hydroxybenzoate (57 mg, $0.25 \mathrm{mmol}, 1$ equiv) and 5-methylhex-4-en-1-ol (34 mg, $0.3 \mathrm{mmol}$, 1.2 equiv) as a white solid in $93 \%$ yield $(64 \mathrm{mg})$.

\section{Characterization data for $\mathbf{3 j}$ :}

$\mathbf{R}_{\mathbf{f}}=0.18$ in hexanes/EtOAc 90:10 v/v.

$[\boldsymbol{\alpha}]_{\mathrm{D}}^{\mathbf{2 0}}=-3.4\left(\mathrm{c} 0.5, \mathrm{CHCl}_{3}, 95 \%\right.$ ee $)$.

${ }^{1}$ H-NMR $\left(400 \mathrm{MHz}, \mathrm{CDCl}_{3}\right): \delta=8.12(\mathrm{dd}, J=2.2,1.1 \mathrm{~Hz}, 1 \mathrm{H}), 7.83(\mathrm{ddd}, J=8.7,2.3,0.7 \mathrm{~Hz}, 1 \mathrm{H}), 6.77(\mathrm{~d}, J=8.6$ $\mathrm{Hz}, 1 \mathrm{H}), 4.21-4.11$ (comp, $2 \mathrm{H}), 3.85(\mathrm{~s}, 3 \mathrm{H}), 3.68-3.59(\mathrm{~m}, 1 \mathrm{H}), 1.97-1.88(\mathrm{~m}, 1 \mathrm{H}), 1.79-1.63(\mathrm{comp}, 3 \mathrm{H}), 1.40(\mathrm{~s}$, $3 \mathrm{H}), 1.38-1.27$ (m, 1H), 1.19 (s, 3H).

${ }^{13}$ C-NMR $\left(100 \mathrm{MHz}, \mathrm{CDCl}_{3}\right): \delta=167.1,157.0,130.7,128.5,122.5,121.9,116.9,79.7,73.0,68.3,51.8,44.9,27.5$, $26.2,25.1,20.8$.

HRMS (ESI-TOF): Calculated for $\mathrm{C}_{16} \mathrm{H}_{21} \mathrm{O}_{4}[\mathrm{M}+\mathrm{H}]^{+}: 277.1434$, found 277.1417.

SFC: Daicel Chiralcel OJ-H, column temperature $=40^{\circ} \mathrm{C}, \mathrm{CO}_{2} / \mathrm{MeOH}=97 / 3$, Flow rate $=2 \mathrm{~mL} / \mathrm{min}, \mathrm{UV}=280 \mathrm{~nm}$, $\mathrm{t}_{\mathrm{R}}=4.8 \mathrm{~min}$ (major) and $\mathrm{t}_{\mathrm{R}}=5.2 \mathrm{~min}$ (minor).

The absolute configuration was assigned by analogy. 
(4aS,10bS)-5,5-Dimethyl-3,4,4a,10b-tetrahydro-2H,5H-pyrano[3,2-c]chromene

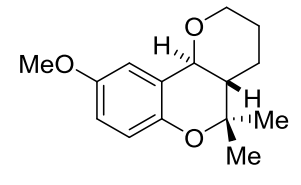

$3 \mathbf{k}$

Following general procedure $\mathrm{C}$ (reaction performed at $\mathrm{rt}$ for $0.5 \mathrm{~h}$ ), compound $\mathbf{3 k}$ was obtained from 5methoxysalicylaldehyde dimethyl acetal $(50 \mathrm{mg}, 0.25 \mathrm{mmol}, 1$ equiv) and 5-methylhex-4-en-1-ol (34 mg, $0.3 \mathrm{mmol}$, 1.2 equiv) as a white solid in $94 \%$ yield $(58 \mathrm{mg})$.

\section{Characterization data for $3 \mathrm{k}$ :}

$\mathbf{R}_{\mathbf{f}}=0.25$ in hexanes/EtOAc $90: 10 \mathrm{v} / \mathrm{v}$.

$[\alpha]_{\mathbf{D}}^{20}=-54.24\left(\mathrm{c} 0.5, \mathrm{CHCl}_{3}, 96 \%\right.$ ee $)$.

${ }^{1} \mathbf{H}-\mathbf{N M R}\left(400 \mathrm{MHz}, \mathrm{CDCl}_{3}\right): \delta=6.98-6.94(\mathrm{~m}, 1 \mathrm{H}), 6.75(\mathrm{ddd}, J=8.8,3.0,0.8 \mathrm{~Hz}, 1 \mathrm{H}), 6.69(\mathrm{~d}, J=8.9 \mathrm{~Hz}, 1 \mathrm{H})$, 4.19-4.12 (comp, 2H), 3.76 (s, 3H), 3.64 (app td, $J=11.4,3.6 \mathrm{~Hz}, 1 \mathrm{H}), 1.92(\operatorname{app} \mathrm{dtd}, J=12.2,3.4,1.5 \mathrm{~Hz}, 1 \mathrm{H})$, 1.81-1.66 (comp, 3H), 1.37 (s, 3H), 1.36-1.24 (m, 1H), 1.17 (s, 3H).

${ }^{13}$ C-NMR $\left(100 \mathrm{MHz}, \mathrm{CDCl}_{3}\right): \delta=153.4,146.8,122.8,117.7,116.1,109.7,78.1,73.9,68.5,55.9,45.3,27.7,26.4$, $25.2,20.3$.

HRMS (ESI-TOF): Calculated for $\mathrm{C}_{15} \mathrm{H}_{21} \mathrm{O}_{3}[\mathrm{M}+\mathrm{H}]^{+}: 249.1485$, found 249.1485 .

SFC: Daicel Chiralcel OJ-H, column temperature $=40^{\circ} \mathrm{C}, \mathrm{CO}_{2} / \mathrm{MeOH}=90 / 10$, Flow rate $=2 \mathrm{~mL} / \mathrm{min}, \mathrm{UV}=230$ $\mathrm{nm}, \mathrm{t}_{\mathrm{R}}=3.1 \min$ (major) and $\mathrm{t}_{\mathrm{R}}=3.8 \min$ (minor).

The absolute configuration was assigned by analogy. 
(4aS,10bS)-5,5-Dimethyl-3,4,4a,10b-tetrahydro-2H,5H-pyrano[3,2-c]chromene

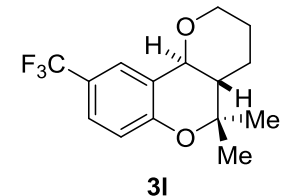

Following general procedure $\mathrm{C}$ (reaction performed at $\mathrm{rt}$ for $4.5 \mathrm{~h}$ ), compound 3l was obtained from 5trifluoromethylsalicylaldehyde dimethyl acetal (59 mg, $0.25 \mathrm{mmol}, 1$ equiv) and 5-methylhex-4-en-1-ol (34 mg, 0.3 mmol, 1.2 equiv) as a white solid in $93 \%$ yield $(67 \mathrm{mg})$.

\section{Characterization data for 3I:}

$\mathbf{R}_{\mathbf{f}}=0.41$ in hexanes/EtOAc $90: 10 \mathrm{v} / \mathrm{v}$.

$[\alpha]_{\mathrm{D}}^{\mathbf{2 0}}=-36.12\left(\mathrm{c} 0.5, \mathrm{CHCl}_{3},>99 \%\right.$ ee $)$

${ }^{1}$ H-NMR $\left(400 \mathrm{MHz}, \mathrm{CDCl}_{3}\right): \delta=7.77-7.68(\mathrm{~m}, 1 \mathrm{H}), 7.42-7.37(\mathrm{~m}, 1 \mathrm{H}), 6.83(\mathrm{dd}, J=8.6,1.0 \mathrm{~Hz}, 1 \mathrm{H}), 4.23-4.13$ (comp, 2H), 3.70-3.60 (m, 1H), 1.98-1.91 (m, 1H), 1.82-1.66 (comp, 3H), 1.41 (s, 3H), 1.40-1.31 (m, 1H), 1.21 (s, $3 \mathrm{H})$.

${ }^{13}$ C-NMR $\left(100 \mathrm{MHz}, \mathrm{CDCl}_{3}\right): \delta=155.6,126.0\left(\mathrm{q}, J_{C-F}=3.7 \mathrm{~Hz}\right), 124.7\left(\mathrm{q}, J_{C-F}=271.1 \mathrm{~Hz}\right), 123.9\left(\mathrm{q}, J_{C-F}=3.9\right.$ $\mathrm{Hz}), 122.9,122.2\left(\mathrm{q}, J_{C-F}=32.6 \mathrm{~Hz}\right), 117.2,79.6,73.0,68.4,44.9,27.6,26.2,25.1,20.7$.

${ }^{19}$ F-NMR $\left(565 \mathrm{MHz}, \mathrm{CDCl}_{3}\right): \delta=-61.38$.

HRMS (ESI-TOF): Calculated for $\mathrm{C}_{15} \mathrm{H}_{18} \mathrm{~F}_{3} \mathrm{O}_{2}[\mathrm{M}+\mathrm{H}]^{+}:$287.1253, found 287.1237.

HPLC: Daicel Chiralpak OJ-H, $n$-hexane $/ i$-PrOH $=90 / 10$, Flow rate $=1 \mathrm{~mL} / \mathrm{min}, \mathrm{UV}=280 \mathrm{~nm}, \mathrm{t}_{\mathrm{R}}=6.4 \mathrm{~min}$ (major).

The absolute configuration was assigned by analogy. 
(4aS,10bS)-5,5-Dimethyl-3,4,4a,10b-tetrahydro-2H,5H-pyrano[3,2-c]chromene

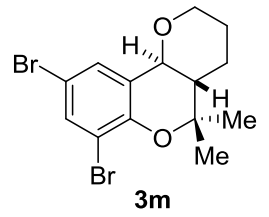

Following general procedure $\mathrm{C}$ (reaction performed at $\mathrm{rt}$ for $20 \mathrm{~h}$ ), compound $\mathbf{3 m}$ was obtained from 3,5dibromosalicylaldehyde dimethyl acetal (82 mg, $0.25 \mathrm{mmol}, 1$ equiv) and 5-methylhex-4-en-1-ol (34 mg, $0.3 \mathrm{mmol}$, 1.2 equiv) as a white solid in $96 \%$ yield $(90 \mathrm{mg})$.

\section{Characterization data for $3 \mathrm{~m}$ :}

$\mathbf{R}_{\mathbf{f}}=0.41$ in hexanes/EtOAc $90: 10 \mathrm{v} / \mathrm{v}$.

$[\alpha]_{\mathbf{D}}^{\mathbf{2 0}}=-1.08\left(\mathrm{c} 0.5, \mathrm{CHCl}_{3}, 91 \%\right.$ ee $)$

${ }^{1}$ H-NMR $\left(400 \mathrm{MHz}_{\mathrm{CDCl}}\right.$ ): $\delta=7.53(\mathrm{dd}, J=2.4,0.8 \mathrm{~Hz}, 1 \mathrm{H}), 7.50(\mathrm{dd}, J=2.4,1.1 \mathrm{~Hz}, 1 \mathrm{H}), 4.20-4.10$ (comp, 2H), 3.66-3.56 (m, 1H), 1.98-1.90 (m, 1H), 1.81-1.64 (comp, 3H), 1.46 (s, 3H), 1.40-1.27 (m, $1 \mathrm{H}), 1.20$ (s, 3H).

${ }^{13}$ C-NMR $\left(100 \mathrm{MHz}, \mathrm{CDCl}_{3}\right): \delta=149.0,134.6,128.3,125.7,112.0,111.6,80.4,73.1,68.5,44.7,27.5,26.1,25.0$, 20.9 .

HRMS (ESI-TOF): Calculated for $\mathrm{C}_{14} \mathrm{H}_{17}{ }^{79} \mathrm{Br}^{81} \mathrm{BrO}_{2}[\mathrm{M}+\mathrm{H}]^{+}: 376.9569$, found 376.9498 .

SFC: Daicel Chiralcel AD-H, column temperature $=40{ }^{\circ} \mathrm{C}, \mathrm{CO}_{2} / \mathrm{MeOH}=90 / 10$, Flow rate $=2 \mathrm{~mL} / \mathrm{min}, \mathrm{UV}=230$ $\mathrm{nm}, \mathrm{t}_{\mathrm{R}}=5.0 \mathrm{~min}$ (minor) and $\mathrm{t}_{\mathrm{R}}=5.3 \min$ (major).

The absolute configuration was assigned by analogy. 


\section{$(4 a S, 10 b S)-5,5-D i m e t h y l-3,4,4 a, 10 b$-tetrahydro-2H,5H-pyrano[3,2-c]chromene}

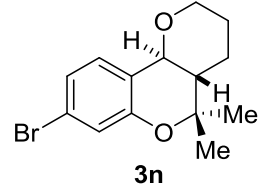

Following general procedure $\mathrm{C}$ (reaction performed at $0{ }^{\circ} \mathrm{C}$ for $3.5 \mathrm{~h}$ ), compound 3n was obtained from 4bromosalicylaldehyde dimethyl acetal (62 mg, $0.25 \mathrm{mmol}, 1$ equiv) and 5-methylhex-4-en-1-ol (34 mg, $0.3 \mathrm{mmol}$, 1.2 equiv) as a white solid in $92 \%$ yield $(68 \mathrm{mg})$.

\section{Characterization data for $3 \mathrm{n}$ :}

$\mathbf{R}_{\mathbf{f}}=0.35$ in hexanes/EtOAc $90: 10 \mathrm{v} / \mathrm{v}$.

$[\boldsymbol{\alpha}]_{\mathrm{D}}^{20}=-56.06\left(\mathrm{c} 0.5, \mathrm{CHCl}_{3}, 94 \%\right.$ ee $)$

${ }^{1}$ H-NMR $\left(600 \mathrm{MHz}, \mathrm{CDCl}_{3}\right): \delta=7.59(\mathrm{~d}, J=8.1 \mathrm{~Hz}, 1 \mathrm{H}), 7.33(\mathrm{dd}, J=8.3,2.0 \mathrm{~Hz}, 1 \mathrm{H}), 7.26(\mathrm{~d}, J=2.1 \mathrm{~Hz}, 1 \mathrm{H})$, 4.49-4.39 (comp, 2H), 3.95 (app td, $J=11.6,3.5 \mathrm{~Hz}, 1 \mathrm{H}), 2.27-2.21$ (m, 1H), 2.14-2.01 (comp, $2 \mathrm{H}), 1.99$ (ddd, $J=$ $13.5,10.7,3.3 \mathrm{~Hz}, 1 \mathrm{H}), 1.70(\mathrm{~s}, 3 \mathrm{H}), 1.64$ (app tt, $J=12.3,6.1 \mathrm{~Hz}, 1 \mathrm{H}), 1.50(\mathrm{~s}, 3 \mathrm{H})$.

${ }^{13}$ C-NMR $\left(150 \mathrm{MHz}, \mathrm{CDCl}_{3}\right): \delta=153.8,127.6,123.1,121.9,121.8,119.9,79.3,73.3,68.4,45.0,27.6,26.3,25.1$, 20.6 .

HRMS (ESI-TOF): Calculated for $\mathrm{C}_{14} \mathrm{H}_{18}{ }^{79} \mathrm{BrO}_{2}[\mathrm{M}+\mathrm{H}]^{+}:$297.0485, found 297.0423.

SFC: Daicel Chiralcel AD-H, column temperature $=40{ }^{\circ} \mathrm{C}, \mathrm{CO}_{2} / \mathrm{MeOH}=95 / 5$, Flow rate $=2 \mathrm{~mL} / \mathrm{min}, \mathrm{UV}=280$ $\mathrm{nm}, \mathrm{t}_{\mathrm{R}}=7.2 \min$ (minor) and $\mathrm{t}_{\mathrm{R}}=10.4 \min$ (major).

The absolute configuration of $\mathbf{3 n}$ was assigned by X-ray crystallography:

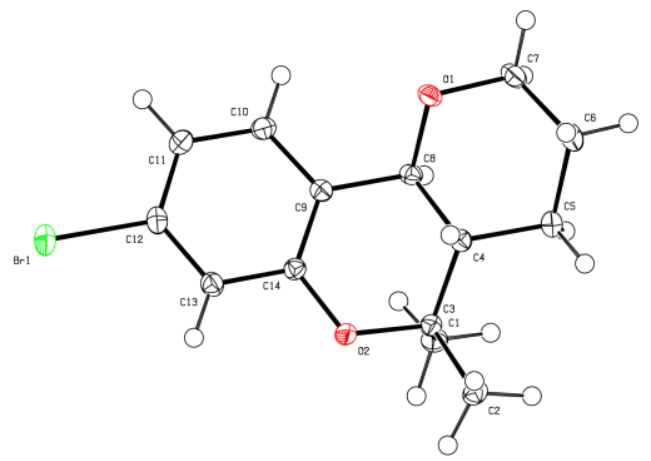

Compound 3n was crystallized from $\mathrm{CH}_{2} \mathrm{Cl}_{2} /$ hexane through slow evaporation at $4{ }^{\circ} \mathrm{C}$. The requisite $\mathrm{CIF}$ has been deposited with the CCDC (deposition \# 2010290). 
(4aS,10bS)-5,5-Dimethyl-3,4,4a,10b-tetrahydro-2H,5H-pyrano[3,2-c]chromene

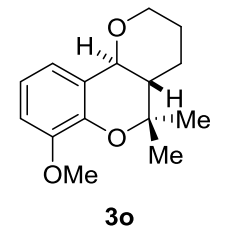

Following general procedure $\mathrm{C}$ (reaction performed at $-30{ }^{\circ} \mathrm{C}$ for $12 \mathrm{~h}$ ), compound 3o was obtained from 3methoxysalicylaldehyde dimethyl acetal (50 mg, $0.25 \mathrm{mmol}, 1$ equiv) and 5-methylhex-4-en-1-ol (34 mg, $0.3 \mathrm{mmol}$, 1.2 equiv) as a white solid in $89 \%$ yield $(55 \mathrm{mg})$.

\section{Characterization data for 3o:}

$\mathbf{R}_{\mathbf{f}}=0.15$ in hexanes/EtOAc $90: 10 \mathrm{v} / \mathrm{v}$.

$[\boldsymbol{\alpha}]_{\mathrm{D}}^{20}=-27.26\left(\mathrm{c} 0.5, \mathrm{CHCl}_{3}, 98 \%\right.$ ee $)$

${ }^{1}$ H-NMR $\left(400 \mathrm{MHz} \mathrm{CDCl}_{3}\right): \delta=7.05(\mathrm{ddd}, J=7.7,1.6,0.9 \mathrm{~Hz}, 1 \mathrm{H}), 6.85($ app t, $J=7.9 \mathrm{~Hz}, 1 \mathrm{H}), 6.78(\mathrm{ddd}, J=$ 8.0, 1.7, $0.7 \mathrm{~Hz}, 1 \mathrm{H}), 4.20(\mathrm{~d}, J=10.5 \mathrm{~Hz}, 1 \mathrm{H}), 4.18-4.12(\mathrm{~m}, 1 \mathrm{H}), 3.84(\mathrm{~s}, 3 \mathrm{H}), 3.64(\operatorname{app~td}, J=11.5,3.5 \mathrm{~Hz}, 1 \mathrm{H})$, 1.98-1.90 (m, 1H), 1.85-1.68 (comp, 3H), 1.48 (s, 3H), 1.33 (app qd, $J=12.3,4.9 \mathrm{~Hz}, 1 \mathrm{H}), 1.23$ (s, $3 \mathrm{H})$.

${ }^{13}$ C-NMR $\left(100 \mathrm{MHz}, \mathrm{CDCl}_{3}\right): \delta=148.3,142.5,123.4,119.5,118.0,110.8,78.9,73.7,68.4,56.2,45.0,27.6,26.3$, $25.2,20.4$.

HRMS (ESI-TOF): Calculated for $\mathrm{C}_{15} \mathrm{H}_{21} \mathrm{O}_{3}[\mathrm{M}+\mathrm{H}]^{+}:$249.1485, found 249.1477.

SFC: Daicel Chiralcel OJ-H, column temperature $=40{ }^{\circ} \mathrm{C}, \mathrm{CO}_{2} / \mathrm{MeOH}=80 / 20$, Flow rate $=2 \mathrm{~mL} / \mathrm{min}, \mathrm{UV}=230$ $\mathrm{nm}, \mathrm{t}_{\mathrm{R}}=3.7 \mathrm{~min}$ (minor) and $\mathrm{t}_{\mathrm{R}}=4.6 \mathrm{~min}$ (major).

The absolute configuration was assigned by analogy. 


\section{$(4 a S, 10 b S)-5,5-D i m e t h y l-3,4,4 a, 10 b$-tetrahydro-2H,5H-pyrano[3,2-c]chromene}

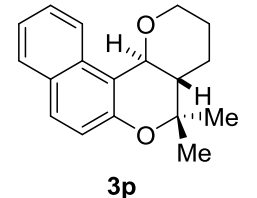

Following general procedure $\mathrm{C}$ (reaction performed at $-30{ }^{\circ} \mathrm{C}$ for $6 \mathrm{~h}$ ), compound $3 \mathbf{p}$ was obtained from 1 (dimethoxymethyl)naphthalen-2-ol (55 mg, $0.25 \mathrm{mmol}, 1$ equiv) and 5-methylhex-4-en-1-ol (34 mg, $0.3 \mathrm{mmol}, 1.2$ equiv) as a white solid in $84 \%$ yield $(63 \mathrm{mg})$.

\section{Characterization data for $3 p$ :}

$\mathbf{R}_{\mathbf{f}}=0.38$ in hexanes/EtOAc $90: 10 \mathrm{v} / \mathrm{v}$.

$[\boldsymbol{\alpha}]_{\mathbf{D}}^{\mathbf{2 0}}=-8.32\left(\mathrm{c} 0.5, \mathrm{CHCl}_{3}, 94 \%\right.$ ee $)$

${ }^{1}$ H-NMR $\left(400 \mathrm{MHz}, \mathrm{CDCl}_{3}\right): \delta=8.23(\mathrm{~d}, J=8.6 \mathrm{~Hz}, 1 \mathrm{H}), 7.76-7.70(\mathrm{~m}, 1 \mathrm{H}), 7.68(\mathrm{~d}, J=8.9 \mathrm{~Hz}, 1 \mathrm{H}), 7.46(\mathrm{ddd}, J$ $=8.5,6.8,1.4 \mathrm{~Hz}, 1 \mathrm{H}), 7.32(\mathrm{ddd}, J=8.1,6.8,1.2 \mathrm{~Hz}, 1 \mathrm{H}), 7.00(\mathrm{~d}, J=8.9 \mathrm{~Hz}, 1 \mathrm{H}), 4.62(\mathrm{~d}, J=9.9 \mathrm{~Hz}, 1 \mathrm{H}), 4.27-$ 4.19 (m, 1H), 3.85 (app td, $J=11.6,3.5 \mathrm{~Hz}, 1 \mathrm{H}$ ), 2.10-1.97 (comp, 2H), 1.95-1.76 (comp, 2H), $1.52-1.38$ (comp, $4 \mathrm{H}), 1.18(\mathrm{~s}, 3 \mathrm{H})$.

${ }^{13}$ C-NMR $\left(100 \mathrm{MHz}, \mathrm{CDCl}_{3}\right): \delta=151.7,132.6,130.2,129.4,128.3,126.1,125.3,123.1,119.4,113.5,78.3,74.7$, $68.5,47.0,27.4,26.7,25.7,19.2$.

HRMS (ESI-TOF): Calculated for $\mathrm{C}_{18} \mathrm{H}_{21} \mathrm{O}_{2}[\mathrm{M}+\mathrm{H}]^{+}: 269.1536$, found 269.1520.

SFC: Daicel Chiralcel OJ-H, column temperature $=40{ }^{\circ} \mathrm{C}, \mathrm{CO}_{2} / \mathrm{MeOH}=80 / 20$, Flow rate $=2 \mathrm{~mL} / \mathrm{min}, \mathrm{UV}=280$ $\mathrm{nm}, \mathrm{t}_{\mathrm{R}}=4.4 \min$ (major) and $\mathrm{t}_{\mathrm{R}}=7.2 \mathrm{~min}$ (minor).

The absolute configuration was assigned by analogy. 
(4aS,10bS)-5,5-Dimethyl-3,4,4a,10b-tetrahydro-2H,5H-pyrano[3,2-c]chromene

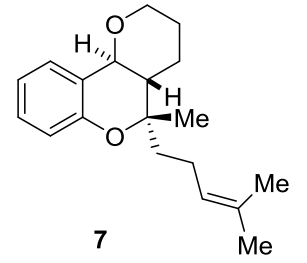

Following general procedure $\mathrm{C}$ (reaction performed at $0{ }^{\circ} \mathrm{C}$ for $3.5 \mathrm{~h}$ ), compound 7 was obtained from salicylaldehyde dimethyl acetal (42 mg, $0.25 \mathrm{mmol}, 1$ equiv) and (Z)-5,9-dimethyldeca-4,8-dien-1-ol (55 mg, 0.3 mmol, 1.2 equiv) as a white solid in $80 \%$ yield $(57 \mathrm{mg})$.

\section{Characterization data for 7:}

$\mathbf{R}_{\mathbf{f}}=0.37$ in hexanes/EtOAc $90: 10 \mathrm{v} / \mathrm{v}$.

$[\alpha]_{\mathrm{D}}^{20}=-16.8\left(\mathrm{c} 0.5, \mathrm{CHCl}_{3}, 92 \%\right.$ ee $)$

${ }^{1}$ H-NMR $\left(500 \mathrm{MHz} \mathrm{CDCl}_{3}\right.$ ): $\delta=7.40$ (app dt, $\left.J=7.6,1.3 \mathrm{~Hz}, 1 \mathrm{H}\right), 7.15$ (dddd, $\left.J=8.1,7.3,1.8,0.8 \mathrm{~Hz}, 1 \mathrm{H}\right), 6.89$ $($ app td, $J=7.5,1.2 \mathrm{~Hz}, 1 \mathrm{H}), 6.78(\mathrm{dd}, J=8.2,1.2 \mathrm{~Hz}, 1 \mathrm{H}), 5.02$ (dddd, $J=7.2,5.8,2.9,1.5 \mathrm{~Hz}, 1 \mathrm{H}), 4.26(\mathrm{~d}, J=$ $10.6 \mathrm{~Hz}, 1 \mathrm{H}), 4.18-4.10(\mathrm{~m}, 1 \mathrm{H}), 3.63$ (app td, $J=11.5,3.5 \mathrm{~Hz}, 1 \mathrm{H}), 2.20-2.03$ (comp, 2H), $1.96-1.88$ (m, 1H), 1.82-1.69 (comp, 3H), 1.67-1.59 (comp, 4H), 1.53 (s, 3H), 1.46-1.33 (comp, 5H).

${ }^{13}$ C-NMR $\left(125 \mathrm{MHz}, \mathrm{CDCl}_{3}\right): \delta=152.9,131.9,129.0,126.3,124.3,122.8,120.1,117.0,80.0,73.4,68.5,46.6,32.5$, $26.7,25.8,25.1,24.2,21.8,17.6$.

HRMS (ESI-TOF): Calculated for $\mathrm{C}_{19} \mathrm{H}_{27} \mathrm{O}_{2}[\mathrm{M}+\mathrm{H}]^{+}:$287.2006, found 287.2024.

SFC: Daicel Chiralcel OJ-H, column temperature $=40{ }^{\circ} \mathrm{C}, \mathrm{CO}_{2} / \mathrm{MeOH}=90 / 10$, Flow rate $=2 \mathrm{~mL} / \mathrm{min}, \mathrm{UV}=230$ $\mathrm{nm}, \mathrm{t}_{\mathrm{R}}=3.3 \mathrm{~min}$ (major) and $\mathrm{t}_{\mathrm{R}}=3.9 \mathrm{~min}$ (minor).

The absolute configuration was assigned by analogy. 
(4aS,10bS)-5,5-Dimethyl-3,4,4a,10b-tetrahydro-2H,5H-pyrano[3,2-c]chromene

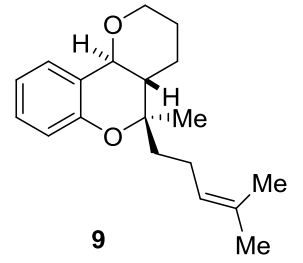

Following general procedure $\mathrm{C}$ (reaction performed at $0{ }^{\circ} \mathrm{C}$ for $3.5 \mathrm{~h}$ ), compound 9 was obtained from salicylaldehyde dimethyl acetal (42 mg, $0.25 \mathrm{mmol}, 1$ equiv) and (E)-5,9-dimethyldeca-4,8-dien-1-ol (55 mg, 0.3 mmol, 1.2 equiv) as a white solid in $89 \%$ yield $(63 \mathrm{mg})$.

\section{Characterization data for 9:}

$\mathbf{R}_{\mathbf{f}}=0.51$ in hexanes/EtOAc $90: 10 \mathrm{v} / \mathrm{v}$.

$[\boldsymbol{\alpha}]_{\mathrm{D}}^{20}=-41.18\left(\mathrm{c} 0.5, \mathrm{CHCl}_{3}, 98 \%\right.$ ee $)$

${ }^{1}$ H-NMR (400 MHz, $\left.\mathrm{CDCl}_{3}\right): \delta=7.42(\operatorname{app~dt}, J=7.6,1.3 \mathrm{~Hz}, 1 \mathrm{H}), 7.16$ (app td, $\left.J=7.5,1.7 \mathrm{~Hz}, 1 \mathrm{H}\right), 6.90$ (app td, $J=7.5,1.2 \mathrm{~Hz}, 1 \mathrm{H}), 6.79(\mathrm{dd}, J=8.2,1.2 \mathrm{~Hz}, 1 \mathrm{H}), 5.20-5.10(\mathrm{~m}, 1 \mathrm{H}), 4.27(\mathrm{~d}, J=10.2 \mathrm{~Hz}, 1 \mathrm{H}), 4.21-4.11(\mathrm{~m}$, 1H), 3.66 (app td, $J=11.4,3.7 \mathrm{~Hz}, 1 \mathrm{H}), 2.27-2.03$ (comp, 2H), 1.94-1.56 (comp, 12H), 1.43-1.27 (m, 1H), 1.19 (s, $3 \mathrm{H})$.

${ }^{13}$ C-NMR $\left(100 \mathrm{MHz} \mathrm{CDCl}_{3}\right): \delta=153.1,131.9,128.9,126.1,124.3,122.6,119.9,116.9,79.7,73.7,68.4,42.2,39.7$, $26.4,25.8,25.0,21.3,20.1,17.8$.

HRMS (ESI-TOF): Calculated for $\mathrm{C}_{19} \mathrm{H}_{27} \mathrm{O}_{2}[\mathrm{M}+\mathrm{H}]^{+}: 287.2006$, found 287.1988.

SFC: Daicel Chiralcel OJ-H, column temperature $=40^{\circ} \mathrm{C}, \mathrm{CO}_{2} / \mathrm{MeOH}=90 / 10$, Flow rate $=2 \mathrm{~mL} / \mathrm{min}, \mathrm{UV}=280$ $\mathrm{nm}, \mathrm{t}_{\mathrm{R}}=3.1 \mathrm{~min}$ (major) and $\mathrm{t}_{\mathrm{R}}=3.8 \mathrm{~min}$ (minor).

The absolute configuration was assigned by analogy. 
(4aS,10bS)-5,5-Dimethyl-3,4,4a,10b-tetrahydro-2H,5H-pyrano[3,2-c]chromene

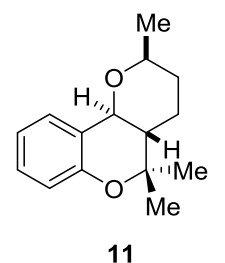

To a flame dried vial was added ( \pm -6-methylhept-5-en-2-ol $(0.114 \mathrm{~mL}, 0.75 \mathrm{mmol}, 3$ equiv), acid catalyst $4 \mathbf{b}$ (10.9 $\mathrm{mg}, 0.0125 \mathrm{mmol}, 5 \mathrm{~mol} \%), 5 \AA \mathrm{MS}(100 \mathrm{mg})$, and dry toluene $(2.5 \mathrm{~mL}, 0.1 \mathrm{M})$. The resulting mixture was stirred under nitrogen and cooled to $-10{ }^{\circ} \mathrm{C}$ over 15 minutes. Salicylaldehyde dimethyl acetal (42 mg, $0.25 \mathrm{mmol}, 1$ equiv) was added and the reaction mixture was stirred for $48 \mathrm{~h}$, then quenched with triethylamine $(0.1 \mathrm{~mL})$. The resulting mixture was directly purified by flash chromatography on silica gel topped with Celite using EtOAc/hexanes/Et ${ }_{3} \mathrm{~N}$ (2/98/0.1) as the eluent. Compound 11 was obtained as a white solid in $71 \%$ yield (41 mg).

\section{Characterization data for 11:}

$\mathbf{R}_{\mathbf{f}}=0.45$ in hexanes/EtOAc $90: 10 \mathrm{v} / \mathrm{v}$.

$[\boldsymbol{\alpha}]_{\mathrm{D}}^{20}=-43.00\left(\mathrm{c} 0.5, \mathrm{CHCl}_{3}, 92 \%\right.$ ee $)$.

${ }^{1}$ H-NMR $\left(500 \mathrm{MHz} \mathrm{CDCl}_{3}\right): \delta=7.46(\operatorname{app~dt}, J=7.7,1.3 \mathrm{~Hz}, 1 \mathrm{H}), 7.14(\mathrm{dddd}, J=8.2,7.3,1.8,0.8 \mathrm{~Hz}, 1 \mathrm{H}), 6.89$ $(\operatorname{app~td}, J=7.5,1.2 \mathrm{~Hz}, 1 \mathrm{H}), 6.76(\mathrm{dd}, J=8.2,1.1 \mathrm{~Hz}, 1 \mathrm{H}), 4.25(\mathrm{~d}, J=10.5 \mathrm{~Hz}, 1 \mathrm{H}), 3.71(\mathrm{dqd}, J=10.5,6.2,2.2$ $\mathrm{Hz}, 1 \mathrm{H}), 1.93-1.86(\mathrm{~m}, 1 \mathrm{H}), 1.82-1.76(\mathrm{~m}, 1 \mathrm{H}), 1.67$ (ddd, $J=11.8,10.5,3.3 \mathrm{~Hz}, 1 \mathrm{H}), 1.47-1.34$ (comp, $5 \mathrm{H}), 1.30$ $(\mathrm{d}, J=6.2 \mathrm{~Hz}, 3 \mathrm{H}), 1.19(\mathrm{~s}, 3 \mathrm{H})$

${ }^{13}$ C-NMR $\left(125 \mathrm{MHz}, \mathrm{CDCl}_{3}\right): \delta=152.9,128.8,126.2,122.8,120.1,116.8,78.5,74.0,73.3,44.8,33.6,27.9,25.5$, $22.0,20.6$.

HRMS (ESI-TOF): Calculated for $\mathrm{C}_{15} \mathrm{H}_{21} \mathrm{O}_{2}[\mathrm{M}+\mathrm{H}]^{+}: 233.1536$, found 233.1531.

SFC: Daicel Chiralcel AD-H, column temperature $=40{ }^{\circ} \mathrm{C}, \mathrm{CO}_{2} / \mathrm{MeOH}=97 / 3$, Flow rate $=2 \mathrm{~mL} / \mathrm{min}$, UV $=280$ $\mathrm{nm}, \mathrm{t}_{\mathrm{R}}=3.2 \mathrm{~min}$ (minor) and $\mathrm{t}_{\mathrm{R}}=3.5 \mathrm{~min}$ (major).

The absolute configuration was assigned by analogy. 
13 and 13':

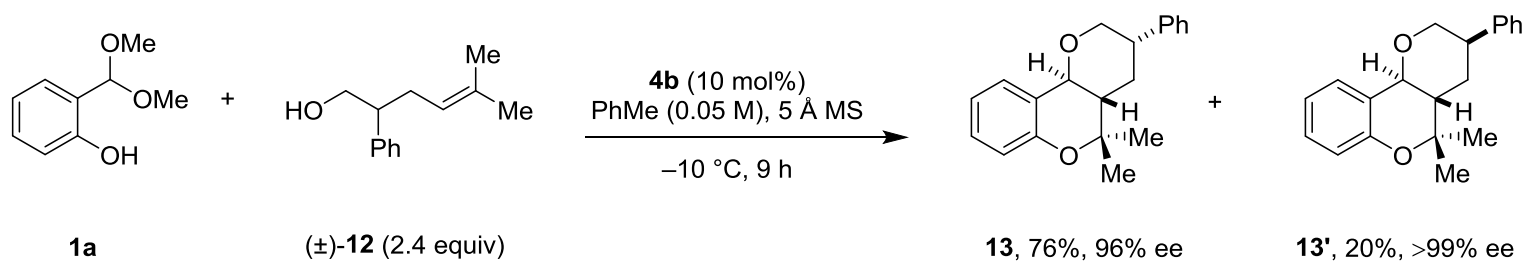

To a flame dried vial was added ( \pm )-5-methyl-2-phenylhex-4-en-1-ol (114 mg, $0.6 \mathrm{mmol}, 2.4$ equiv), acid catalyst 4b $(10.9 \mathrm{mg}, 0.0125 \mathrm{mmol}, 5 \mathrm{~mol} \%), 5 \AA \mathrm{MS}(100 \mathrm{mg})$, and dry toluene $(2.5 \mathrm{~mL}, 0.05 \mathrm{M})$. The resulting mixture was stirred under nitrogen and cooled to $-10{ }^{\circ} \mathrm{C}$ over 15 minutes. Salicylaldehyde dimethyl acetal (42 $\mathrm{mg}, 0.25$ mmol, 1 equiv) was added and the reaction mixture was stirred for $9 \mathrm{~h}$, then quenched with triethylamine $(0.1 \mathrm{~mL})$. The resulting mixture was directly purified by flash chromatography on silica gel topped with Celite using EtOAc/hexanes/Et ${ }_{3} \mathrm{~N}(2 / 98 / 0.1)$ as the eluent. Compounds $\mathbf{1 3}$ and $\mathbf{1 3}{ }^{\prime}$ were obtained as white solids in a 4:1 ratio (71 mg, 95\% combined yield).

Characterization data for major diastereomer 13: a white solid in $75.5 \%$ yield (55.5 mg).

$\mathbf{R}_{\mathbf{f}}=0.37$ in hexanes/EtOAc 90:10 v/v.

$[\alpha]_{\mathrm{D}}^{20}=-52.24\left(\mathrm{c} 0.5, \mathrm{CHCl}_{3}, 96 \%\right.$ ee $)$.

${ }^{1}$ H-NMR $\left(600 \mathrm{MHz}, \mathrm{CDCl}_{3}\right.$ ): $\delta=7.49$ (app dt, $\left.J=7.7,1.3 \mathrm{~Hz}, 1 \mathrm{H}\right), 7.40-7.36$ (comp, 2H), 7.32-7.27 (comp, 3H), 7.21 (dddd, $J=8.1,7.3,1.6,0.7 \mathrm{~Hz}, 1 \mathrm{H}), 6.95($ app td, $J=7.5,1.2 \mathrm{~Hz}, 1 \mathrm{H}), 6.83(\mathrm{dd}, J=8.3,1.2 \mathrm{~Hz}, 1 \mathrm{H}), 4.34(\mathrm{~d}, J$ $=10.6 \mathrm{~Hz}, 1 \mathrm{H}), 4.24(\mathrm{ddd}, J=11.3,4.6,1.8 \mathrm{~Hz}, 1 \mathrm{H}), 3.70($ app t, $J=11.4 \mathrm{~Hz}, 1 \mathrm{H}), 3.06($ app tt, $J=11.7,4.2 \mathrm{~Hz}$, 1H), 2.19-2.14 (m, 1H), 1.95 (ddd, $J=12.1,10.5,3.2 \mathrm{~Hz}, 1 \mathrm{H}), 1.64$ (app q, $J=12.3 \mathrm{~Hz}, 1 \mathrm{H}), 1.45$ (s, 3H), 1.29 (s, $3 \mathrm{H})$.

${ }^{13}$ C-NMR $\left(150 \mathrm{MHz}, \mathrm{CDCl}_{3}\right): \delta=152.9,141.7,129.1,128.8,127.6,127.1,126.2,122.3,120.2,116.9,78.5,73.60$, $73.59,45.3,43.4,32.6,27.8,20.7$.

HRMS (ESI-TOF): Calculated for $\mathrm{C}_{20} \mathrm{H}_{23} \mathrm{O}_{2}[\mathrm{M}+\mathrm{H}]^{+}: 295.1693$, found 295.1694.

SFC: Daicel Chiralcel OJ-H, column temperature $=40^{\circ} \mathrm{C}, \mathrm{CO}_{2} / \mathrm{MeOH}=90 / 10$, Flow rate $=2 \mathrm{~mL} / \mathrm{min}, \mathrm{UV}=280$ $\mathrm{nm}, \mathrm{t}_{\mathrm{R}}=7.0 \mathrm{~min}$ (minor) and $\mathrm{t}_{\mathrm{R}}=8.0 \mathrm{~min}$ (major).

The absolute configuration was assigned by analogy. 
Characterization data for minor diastereomer 13': a white solid in 19.9\% yield (14.6 mg).

$\mathbf{R}_{\mathbf{f}}=0.43$ in hexanes/EtOAc $\left.90: 10 \mathrm{v} / \mathrm{v}\right)$.

$[\boldsymbol{\alpha}]_{\mathbf{D}}^{20}=-3.12\left(\mathrm{c} 0.5, \mathrm{CHCl}_{3},>99 \%\right.$ ee $)$.

${ }^{1}$ H-NMR (600 MHz, CDCl $)$ ): $\delta=7.50-7.45$ (comp, 3H), 7.29-7.24 (comp, 3H), 7.20-7.14 (comp, 2H), 6.93 (app td, $J=7.5,1.2 \mathrm{~Hz}, 1 \mathrm{H}), 6.75(\mathrm{dd}, J=8.2,1.2 \mathrm{~Hz}, 1 \mathrm{H}), 4.53$ (ddd, $J=11.9,2.1,1.0 \mathrm{~Hz}, 1 \mathrm{H}), 4.35-4.30(\mathrm{~m}, 1 \mathrm{H}), 4.09$ (dd, $J=11.9,3.4 \mathrm{~Hz}, 1 \mathrm{H}), 3.09-3.05(\mathrm{~m}, 1 \mathrm{H}), 2.03$ (app dt, $J=9.6,2.2 \mathrm{~Hz}, 1 \mathrm{H}), 1.93-1.84(\mathrm{comp}, 2 \mathrm{H}), 1.23$ (s, $3 \mathrm{H}), 1.21(\mathrm{~s}, 3 \mathrm{H})$.

${ }^{13}$ C-NMR $\left(150 \mathrm{MHz}, \mathrm{CDCl}_{3}\right): \delta=152.9,144.4,129.0,128.5,128.4,126.5,126.1,122.7,120.0,116.9,78.4,74.2$, $70.8,39.4,38.7,32.5,27.4,20.5$.

HRMS (ESI-TOF): Calculated for $\mathrm{C}_{20} \mathrm{H}_{23} \mathrm{O}_{2}[\mathrm{M}+\mathrm{H}]^{+}: 295.1693$, found 295.1709.

SFC: Daicel Chiralcel AD-H, column temperature $=40{ }^{\circ} \mathrm{C}, \mathrm{CO}_{2} / \mathrm{MeOH}=90 / 10$, Flow rate $=2 \mathrm{~mL} / \mathrm{min}, \mathrm{UV}=210$ $\mathrm{nm}, \mathrm{t}_{\mathrm{R}}=3.4 \min$ (major).

The absolute configuration was assigned by analogy.

Note: Racemic $\mathbf{1 3}$ and $\mathbf{1 3}$ ' were prepared on a $5 \mathrm{mmol}$ scale due to the high diastereoselectivity of the reaction (to obtain a sufficient amount of the minor diastereomer).

A flame dried $100 \mathrm{~mL}$ round bottom flask was charged with $( \pm)$-5-methyl-2-phenylhex-4-en-1-ol (1.14 mg, $6 \mathrm{mmol}$, 1.2 equiv), salicylaldehyde $(0.533 \mathrm{~mL}, 5 \mathrm{mmol}, 1$ equiv), toluene-4-sulfonic acid monohydrate (190 mg, $1 \mathrm{mmol}, 20$ mol\%) and dry toluene $(50 \mathrm{~mL}, 0.1 \mathrm{M})$. Trimethyl orthoformate $(0.685 \mathrm{~mL}, 6 \mathrm{mmol}, 1.2$ equiv) was added slowly. The resulting mixture was stirred at room temperature for $2 \mathrm{~h}$, and then directly purified by flash chromatography on silica gel using EtOAc/hexanes/ $\mathrm{Et}_{3} \mathrm{~N}$ (2/98/0.1) as the eluent. Racemic 13 was obtained as white solid in $84 \%$ yield $(1.23 \mathrm{~g})$ and racemic $\mathbf{1 3}^{\prime}$ was obtained as white solid in $0.34 \%$ yield $(5 \mathrm{mg})$. 
(4aS,10bS)-5,5-Dimethyl-3,4,4a,10b-tetrahydro-2H,5H-pyrano[3,2-c]chromene

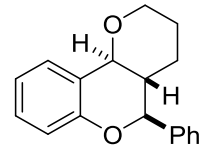

S2

Following general procedure C (reaction performed at $\mathrm{rt}$ for $4.5 \mathrm{~h}$ ), compound $\mathbf{S 2}$ was obtained from salicylaldehyde dimethyl acetal (42 mg, $0.25 \mathrm{mmol}, 1$ equiv) and (E)-5-phenylpent-4-en-1-ol (49 mg, $0.3 \mathrm{mmol}, 1.2$ equiv) as a white solid in $83 \%$ yield $(55 \mathrm{mg})$.

\section{Characterization data for S2:}

$\mathbf{R}_{\mathbf{f}}=0.48$ in hexanes/EtOAc 90:10 v/v.

$[\boldsymbol{\alpha}]_{\mathbf{D}}^{\mathbf{2 0}}=-20.50\left(\mathrm{c} 0.5, \mathrm{CHCl}_{3}, 31 \%\right.$ ee $)$.

${ }^{1}$ H-NMR $\left(500 \mathrm{MHz}, \mathrm{CDCl}_{3}\right): \delta=7.46(\mathrm{dt}, J=7.7,1.4 \mathrm{~Hz}, 1 \mathrm{H}), 7.45-7.33$ (comp, 5H), 7.19 (dddd, $J=8.2,7.4,1.7$, $0.8 \mathrm{~Hz}, 1 \mathrm{H}), 6.96$ (app td, $J=7.5,1.2 \mathrm{~Hz}, 1 \mathrm{H}), 6.86(\mathrm{dd}, J=8.3,1.2 \mathrm{~Hz}, 1 \mathrm{H}), 4.85(\mathrm{~d}, J=10.6 \mathrm{~Hz}, 1 \mathrm{H}), 4.44-4.38$ (m, 1H), 4.22-4.15 (m, 1H), 3.67 (app td, $J=11.6,3.3 \mathrm{~Hz}, 1 \mathrm{H}$ ), 1.97 (app dtd, $J=11.9,10.3,3.5 \mathrm{~Hz}, 1 \mathrm{H}$ ), $1.74-$ 1.58 (comp, 2H), 1.42-1.33 (m, 1H), 1.19 (app qd, $J=12.3,4.8 \mathrm{~Hz}, 1 \mathrm{H}$ ).

${ }^{13}$ C-NMR $\left(125 \mathrm{MHz}, \mathrm{CDCl}_{3}\right): \delta=154.1,138.7,128.9,128.71,128.67,127.4,125.5,123.7,120.7,116.3,82.0,76.6$, $68.5,42.2,25.9,25.8$.

HRMS (ESI-TOF): Calculated for $\mathrm{C}_{18} \mathrm{H}_{19} \mathrm{O}_{2}[\mathrm{M}+\mathrm{H}]^{+}: 267.1380$, found 267.1391.

SFC: Daicel Chiralcel AD-H, column temperature $=40{ }^{\circ} \mathrm{C}, \mathrm{CO}_{2} / \mathrm{MeOH}=90 / 10$, Flow rate $=2 \mathrm{~mL} / \mathrm{min}, \mathrm{UV}=280$ $\mathrm{nm}, \mathrm{t}_{\mathrm{R}}=6.3 \mathrm{~min}$ (minor) and $\mathrm{t}_{\mathrm{R}}=7.5 \mathrm{~min}$ (major).

The absolute configuration was assigned by analogy. 
S4:

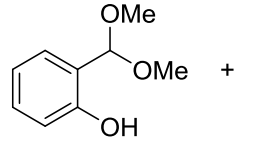

$1 \mathrm{a}$

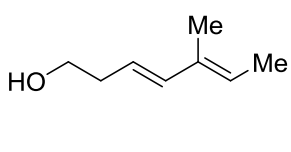

(1.2 equiv)

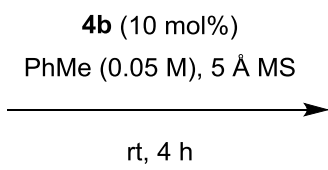

$\mathrm{rt}, 4 \mathrm{~h}$

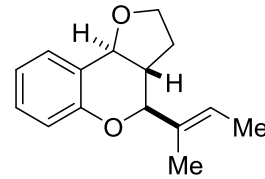

S4, $15 \%$

$\mathrm{dr}=2: 1,34 \% / 33 \%$ ee

Following general procedure $\mathrm{C}$ (reaction performed at $\mathrm{rt}$ for $\mathbf{4 h}$ ), compound $\mathbf{S 4}$ was obtained from salicylaldehyde dimethyl acetal (42 mg, $0.25 \mathrm{mmol}, 1$ equiv) and (3E,5E)-5-methylhepta-3,5-dien-1-ol ( $38 \mathrm{mg}, 0.3 \mathrm{mmol}, 1.2$ equiv) as a mixture of two diastereomers in a 2:1 ratio ( $9 \mathrm{mg}, 15 \%$ combined yield).

The relative configuration of the major diastereomer was confirmed based on literature data. ${ }^{8}$

\section{Characterization data for S4:}

$\mathbf{R}_{\mathbf{f}}=0.39$ in hexanes/EtOAc $90: 10 \mathrm{v} / \mathrm{v}$.

${ }^{1}$ H-NMR $\left(600 \mathrm{MHz}, \mathrm{CDCl}_{3}\right): \delta=7.41(\mathrm{dd}, J=7.7,1.7 \mathrm{~Hz}, 0.47 \mathrm{H}), 7.29-7.26(\mathrm{~m}, 1.14 \mathrm{H}), 7.21(\mathrm{ddd}, J=8.6,7.3$, $1.7 \mathrm{~Hz}, 0.49 \mathrm{H}), 7.18-7.14$ (m, 1.06H), 6.96 (app td, $J=7.4,1.2 \mathrm{~Hz}, 0.51 \mathrm{H}), 6.94-6.85$ (comp, 2.47H), 5.74-5.60 (comp, 1.51H), 4.65-4.55 (comp, 1.48H), $4.46(\mathrm{~d}, J=10.5 \mathrm{~Hz}, 1.00 \mathrm{H}), 4.24$ (ddd, $J=9.8,8.5,2.9 \mathrm{~Hz}, 1.06 \mathrm{H}), 4.19$ (app td, $J=8.6,7.4 \mathrm{~Hz}, 1.11 \mathrm{H}$ ), 3.99 (app td, $J=8.5,5.6 \mathrm{~Hz}, 0.55 \mathrm{H}), 3.88$ (d, $J=11.4 \mathrm{~Hz}, 0.49 \mathrm{H}), 3.82$ (app td, $J=$ $8.9,6.6 \mathrm{~Hz}, 0.55 \mathrm{H}), 2.53-2.47$ (m, 0.51H), 2.21-2.09 (comp, 1.59H), 1.98 (app dtd, $J=11.9,7.2,2.9 \mathrm{~Hz}, 1.13 \mathrm{H})$, $1.80-1.66$ (comp, $10.05 \mathrm{H})$

${ }^{13}$ C-NMR $\left(150 \mathrm{MHz}, \mathrm{CDCl}_{3}\right): \delta=153.9,134.3,131.0,129.5,128.6,126.6,125.5,124.8,123.8,121.6,121.1,121.0$, $120.1,117.2,116.0,86.9,82.7,78.1,74.5,69.3,66.0,44.7,38.0,28.4,27.7,13.53,13.48,11.4,11.0$.

\section{SFC:}

For the major diastereomer: Daicel Chiralcel AD-H, column temperature $=40{ }^{\circ} \mathrm{C}, \mathrm{CO}_{2} / \mathrm{MeOH}=90 / 10$, Flow rate $=$ $2 \mathrm{~mL} / \mathrm{min}, \mathrm{UV}=280 \mathrm{~nm}, \mathrm{t}_{\mathrm{R}}=3.9 \mathrm{~min}$ (major) and $\mathrm{t}_{\mathrm{R}}=5.0 \mathrm{~min}$ (minor).

For the minor diastereomer: Daicel Chiralcel OJ-H, column temperature $=40^{\circ} \mathrm{C}, \mathrm{CO}_{2} / \mathrm{MeOH}=90 / 10$, Flow rate $=1$ $\mathrm{mL} / \mathrm{min}, \mathrm{UV}=280 \mathrm{~nm}, \mathrm{t}_{\mathrm{R}}=7.6 \mathrm{~min}$ (major) and $\mathrm{t}_{\mathrm{R}}=7.9 \mathrm{~min}$ (minor). 


\section{D-NMR Analysis of 13 and 13'}

2D-NMR Analysis for major diastereomer 13, Selected Interactions (in $\mathrm{CDCl}_{3}$ )
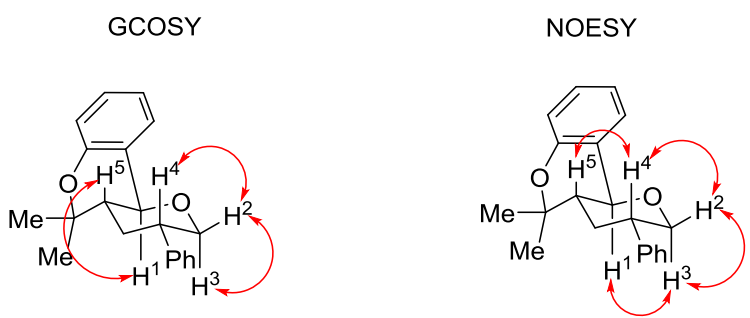

\begin{tabular}{|l|l|}
\hline Protons & Chemical Shifts (ppm) \\
\hline $\mathrm{H}^{1}$ & 4.34 \\
\hline $\mathrm{H}^{2}$ & 4.24 \\
\hline $\mathrm{H}^{3}$ & 3.70 \\
\hline $\mathrm{H}^{4}$ & $3.09-3.05$ \\
\hline $\mathrm{H}^{5}$ & 1.95 \\
\hline
\end{tabular}

2D-NMR Analysis for minor diastereomer 13', Selected Interactions (in $\mathrm{CDCl}_{3}$ )

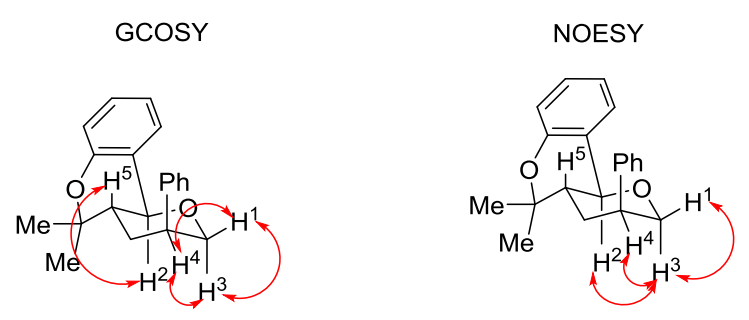

\begin{tabular}{|l|l|}
\hline Protons & Chemical Shifts (ppm) \\
\hline $\mathrm{H}^{1}$ & 4.53 \\
\hline $\mathrm{H}^{2}$ & $4.35-4.30$ \\
\hline $\mathrm{H}^{3}$ & 4.09 \\
\hline $\mathrm{H}^{4}$ & 3.09 \\
\hline $\mathrm{H}^{5}$ & $1.93-1.84$ \\
\hline
\end{tabular}




\section{Crystallographic Summary of Compound 3n}

X-Ray experimental: X-Ray Intensity data were collected at $100 \mathrm{~K}$ on a Bruker D8 Venture diffractometer using MoK $\alpha$ radiation $(\lambda=0.71073 \AA)$ and a Photon III area detector.

Raw data frames were read by program SAINT ${ }^{1}$ and integrated using 3D profiling algorithms. The resulting data were reduced to produce hkl reflections and their intensities and estimated standard deviations. The data were corrected for Lorentz and polarization effects and numerical absorption corrections were applied based on indexed and measured faces.

The structure was solved and refined in SHELXTL2014, using full-matrix least-squares refinement. The non-H atoms were refined with anisotropic thermal parameters and all of the $\mathrm{H}$ atoms were calculated in idealized positions and refined riding on their parent atoms. The asymmetric unit consists of the fused three ring system as shown in the ellipsoids drawing. The absolute configurations is confirmed by anomalous dispersion effects of the $\mathrm{Br}$ atom. Specifically the two protons on $\mathrm{C} 4$ and $\mathrm{C} 8$ are present on opposite sides of the average fused ring plane. In the final cycle of refinement, 4528 reflections (of which 4375 are observed with $\mathrm{I}>2 \sigma(\mathrm{I})$ ) were used to refine 156 parameters and the resulting $\mathrm{R}_{1}, \mathrm{wR}_{2}$ and $\mathrm{S}$ (goodness of fit) were $1.73 \%, 4.25 \%$ and 1.050 , respectively. The refinement was carried out by minimizing the $w_{2}$ function using $F^{2}$ rather than $F$ values. $R_{1}$ is calculated to provide a reference to the conventional $\mathrm{R}$ value but its function is not minimized.

SHELXTL2014 (2014). Bruker-AXS, Madison, Wisconsin, USA. 
Table S1. Crystal data and structure refinement for zhu3.

Identification code

Empirical formula

Formula weight

Temperature

Wavelength

Crystal system

Space group

Unit cell dimensions

Volume

Z

Density (calculated)

Absorption coefficient

$\mathrm{F}(000)$

Crystal size

Theta range for data collection

Index ranges

Reflections collected

Independent reflections

Completeness to theta $=25.000^{\circ}$

Absorption correction

Refinement method

Data / restraints / parameters

Goodness-of-fit on $\mathrm{F}^{2}$

Final R indices [I $>2 \operatorname{sigma}(\mathrm{I})]$

$\mathrm{R}$ indices (all data)

Absolute structure parameter

Extinction coefficient

Largest diff. peak and hole hkl

C14 H17 Br O2

297.18

100(2) K

$0.71073 \AA$

Orthorhombic

P 212121

$\mathrm{a}=9.2472(3) \AA$

$\alpha=90^{\circ}$.

$\mathrm{b}=11.8497(3) \AA$

$\beta=90^{\circ}$.

$\mathrm{c}=11.9375(4) \AA$

$\gamma=90^{\circ}$.
4

$1.509 \mathrm{Mg} / \mathrm{m}^{3}$

$3.130 \mathrm{~mm}^{-1}$

608

$0.272 \times 0.154 \times 0.148 \mathrm{~mm}^{3}$

2.422 to $32.969^{\circ}$.

$-13 \leq \mathrm{h} \leq 13,-17 \leq \mathrm{k} \leq 17,-18 \leq \mathrm{l} \leq 18$

34871

$4528[\mathrm{R}(\mathrm{int})=0.0206]$

$99.0 \%$

None

Full-matrix least-squares on $\mathrm{F}^{2}$

4528 / 0 / 156

1.050

$\mathrm{R} 1=0.0173, \mathrm{wR} 2=0.0425[4375]$

$\mathrm{R} 1=0.0186, \mathrm{wR} 2=0.0430$

$-0.0048(17)$

$\mathrm{n} / \mathrm{a}$

0.290 and -0.409 e. $\AA^{-3}$

$$
\begin{aligned}
& R 1=\sum\left(|| F_{O}|-| F_{C} \mid\right) / \sum\left|F_{O}\right| \quad w R 2=\left[\sum\left[w\left(F_{O}{ }^{2}-F_{C}{ }^{2}\right)^{2}\right] / \sum\left[w\left(F_{O}{ }^{2}\right)^{2}\right]\right]^{1 / 2} \\
& S=\left[\sum\left[w\left(F_{O}{ }^{2}-F_{C}{ }^{2}\right)^{2}\right] /(n-p)\right]^{1 / 2} \quad w=1 /\left[\sigma^{2}\left(F_{O}{ }^{2}\right)+(m * p)^{2}+n * p\right], p=\left[\max \left(F_{O}{ }^{2}, 0\right)+2^{*} F_{C}{ }^{2}\right] / 3, m \text { \& n are constants. }
\end{aligned}
$$


Table S2. Atomic coordinates ( $\left.\times 10^{4}\right)$ and equivalent isotropic displacement parameters $\left(\AA^{2} \times 10^{3}\right)$ for zhu3. $U(e q)$ is defined as one third of the trace of the orthogonalized $U^{i j}$ tensor.

\begin{tabular}{lcccc}
\hline & $\mathrm{x}$ & $\mathrm{y}$ & $\mathrm{z}$ & $\mathrm{U}(\mathrm{eq})$ \\
\hline Br1 & $1490(1)$ & $-115(1)$ & $5493(1)$ & $27(1)$ \\
O1 & $1140(1)$ & $5243(1)$ & $3590(1)$ & $16(1)$ \\
O2 & $3446(1)$ & $2506(1)$ & $2380(1)$ & $16(1)$ \\
C1 & $2257(2)$ & $3168(1)$ & $699(1)$ & $17(1)$ \\
C2 & $4857(2)$ & $3566(1)$ & $1128(1)$ & $18(1)$ \\
C3 & $3343(1)$ & $3471(1)$ & $1610(1)$ & $14(1)$ \\
C4 & $2931(1)$ & $4515(1)$ & $2288(1)$ & $13(1)$ \\
C5 & $2944(2)$ & $5628(1)$ & $1641(1)$ & $17(1)$ \\
C6 & $2479(2)$ & $6581(1)$ & $2427(1)$ & $20(1)$ \\
C7 & $1060(2)$ & $6298(1)$ & $3002(1)$ & $19(1)$ \\
C8 & $1450(2)$ & $4350(1)$ & $2826(1)$ & $13(1)$ \\
C9 & $1406(2)$ & $3247(1)$ & $3446(1)$ & $13(1)$ \\
C10 & $404(2)$ & $3047(1)$ & $4296(1)$ & $16(1)$ \\
C11 & $406(2)$ & $2050(1)$ & $4906(1)$ & $18(1)$ \\
C12 & $1431(2)$ & $1240(1)$ & $4642(1)$ & $17(1)$ \\
C13 & $2425(2)$ & $1390(1)$ & $3786(1)$ & $16(1)$ \\
C14 & $2403(1)$ & $3187(1)$ & $13(1)$ \\
& $2408(1)$ & & & \\
\hline
\end{tabular}


Table S3. Bond lengths $[\AA]$ and angles $\left[^{\circ}\right]$ for zhu3.

\begin{tabular}{|c|c|}
\hline $\mathrm{Br} 1-\mathrm{C} 12$ & $1.9006(12)$ \\
\hline $\mathrm{O} 1-\mathrm{C} 8$ & $1.4260(15)$ \\
\hline $\mathrm{O} 1-\mathrm{C} 7$ & $1.4351(17)$ \\
\hline $\mathrm{O} 2-\mathrm{C} 14$ & $1.3650(15)$ \\
\hline $\mathrm{O} 2-\mathrm{C} 3$ & $1.4694(15)$ \\
\hline $\mathrm{C} 1-\mathrm{C} 3$ & $1.5228(18)$ \\
\hline C1-H1A & 0.9800 \\
\hline C1-H1B & 0.9800 \\
\hline $\mathrm{C} 1-\mathrm{H} 1 \mathrm{C}$ & 0.9800 \\
\hline $\mathrm{C} 2-\mathrm{C} 3$ & $1.5182(18)$ \\
\hline $\mathrm{C} 2-\mathrm{H} 2 \mathrm{~A}$ & 0.9800 \\
\hline $\mathrm{C} 2-\mathrm{H} 2 \mathrm{~B}$ & 0.9800 \\
\hline $\mathrm{C} 2-\mathrm{H} 2 \mathrm{C}$ & 0.9800 \\
\hline $\mathrm{C} 3-\mathrm{C} 4$ & $1.5277(17)$ \\
\hline $\mathrm{C} 4-\mathrm{C} 8$ & $1.5246(19)$ \\
\hline $\mathrm{C} 4-\mathrm{C} 5$ & $1.5285(18)$ \\
\hline C4-H4A & 1.0000 \\
\hline $\mathrm{C} 5-\mathrm{C} 6$ & $1.529(2)$ \\
\hline C5-H5A & 0.9900 \\
\hline C5-H5B & 0.9900 \\
\hline $\mathrm{C} 6-\mathrm{C} 7$ & $1.518(2)$ \\
\hline C6-H6A & 0.9900 \\
\hline C6-H6B & 0.9900 \\
\hline C7-H7A & 0.9900 \\
\hline C7-H7B & 0.9900 \\
\hline C8-C9 & $1.5031(16)$ \\
\hline C8-H8A & 1.0000 \\
\hline C9-C10 & $1.3947(18)$ \\
\hline C9-C14 & $1.3984(18)$ \\
\hline $\mathrm{C} 10-\mathrm{C} 11$ & $1.3885(19)$ \\
\hline C10-H10A & 0.9500 \\
\hline $\mathrm{C} 11-\mathrm{C} 12$ & $1.3858(19)$ \\
\hline C11-H11A & 0.9500 \\
\hline C12-C13 & $1.3855(19)$ \\
\hline
\end{tabular}




\begin{tabular}{|c|c|}
\hline C13-C14 & $1.3976(18)$ \\
\hline C13-H13A & 0.9500 \\
\hline $\mathrm{C} 8-\mathrm{O} 1-\mathrm{C} 7$ & $110.10(10)$ \\
\hline $\mathrm{C} 14-\mathrm{O} 2-\mathrm{C} 3$ & $117.75(10)$ \\
\hline C3-C1-H1A & 109.5 \\
\hline C3-C1-H1B & 109.5 \\
\hline H1A-C1-H1B & 109.5 \\
\hline C3-C1-H1C & 109.5 \\
\hline $\mathrm{H} 1 \mathrm{~A}-\mathrm{C} 1-\mathrm{H} 1 \mathrm{C}$ & 109.5 \\
\hline H1B-C1-H1C & 109.5 \\
\hline $\mathrm{C} 3-\mathrm{C} 2-\mathrm{H} 2 \mathrm{~A}$ & 109.5 \\
\hline C3-C2-H2B & 109.5 \\
\hline $\mathrm{H} 2 \mathrm{~A}-\mathrm{C} 2-\mathrm{H} 2 \mathrm{~B}$ & 109.5 \\
\hline $\mathrm{C} 3-\mathrm{C} 2-\mathrm{H} 2 \mathrm{C}$ & 109.5 \\
\hline $\mathrm{H} 2 \mathrm{~A}-\mathrm{C} 2-\mathrm{H} 2 \mathrm{C}$ & 109.5 \\
\hline $\mathrm{H} 2 \mathrm{~B}-\mathrm{C} 2-\mathrm{H} 2 \mathrm{C}$ & 109.5 \\
\hline $\mathrm{O} 2-\mathrm{C} 3-\mathrm{C} 2$ & $103.61(10)$ \\
\hline $\mathrm{O} 2-\mathrm{C} 3-\mathrm{C} 1$ & $107.83(10)$ \\
\hline $\mathrm{C} 2-\mathrm{C} 3-\mathrm{C} 1$ & $110.81(11)$ \\
\hline $\mathrm{O} 2-\mathrm{C} 3-\mathrm{C} 4$ & $108.34(10)$ \\
\hline $\mathrm{C} 2-\mathrm{C} 3-\mathrm{C} 4$ & $111.76(11)$ \\
\hline $\mathrm{C} 1-\mathrm{C} 3-\mathrm{C} 4$ & $113.86(11)$ \\
\hline $\mathrm{C} 8-\mathrm{C} 4-\mathrm{C} 3$ & $110.07(10)$ \\
\hline $\mathrm{C} 8-\mathrm{C} 4-\mathrm{C} 5$ & $109.30(10)$ \\
\hline $\mathrm{C} 3-\mathrm{C} 4-\mathrm{C} 5$ & $115.41(11)$ \\
\hline C8-C4-H4A & 107.2 \\
\hline C3-C4-H4A & 107.2 \\
\hline C5-C4-H4A & 107.2 \\
\hline C4-C5-C6 & $108.91(11)$ \\
\hline C4-C5-H5A & 109.9 \\
\hline C6-C5-H5A & 109.9 \\
\hline C4-C5-H5B & 109.9 \\
\hline C6-C5-H5B & 109.9 \\
\hline $\mathrm{H} 5 \mathrm{~A}-\mathrm{C} 5-\mathrm{H} 5 \mathrm{~B}$ & 108.3 \\
\hline C7-C6-C5 & $110.93(12)$ \\
\hline
\end{tabular}




\begin{tabular}{|c|c|}
\hline C7-C6-H6A & 109.5 \\
\hline C5-C6-H6A & 109.5 \\
\hline C7-C6-H6B & 109.5 \\
\hline C5-C6-H6B & 109.5 \\
\hline H6A-C6-H6B & 108.0 \\
\hline O1-C7-C6 & $111.62(11)$ \\
\hline O1-C7-H7A & 109.3 \\
\hline C6-C7-H7A & 109.3 \\
\hline O1-C7-H7B & 109.3 \\
\hline C6-C7-H7B & 109.3 \\
\hline H7A-C7-H7B & 108.0 \\
\hline O1-C8-C9 & $108.95(10)$ \\
\hline $\mathrm{O} 1-\mathrm{C} 8-\mathrm{C} 4$ & $110.76(10)$ \\
\hline C9-C8-C4 & $110.07(11)$ \\
\hline O1-C8-H8A & 109.0 \\
\hline C9-C8-H8A & 109.0 \\
\hline C4-C8-H8A & 109.0 \\
\hline C10-C9-C14 & $118.70(11)$ \\
\hline $\mathrm{C} 10-\mathrm{C} 9-\mathrm{C} 8$ & $121.62(12)$ \\
\hline C14-C9-C8 & $119.67(12)$ \\
\hline C11-C10-C9 & $121.70(13)$ \\
\hline C11-C10-H10A & 119.2 \\
\hline C9-C10-H10A & 119.2 \\
\hline $\mathrm{C} 12-\mathrm{C} 11-\mathrm{C} 10$ & $118.08(12)$ \\
\hline $\mathrm{C} 12-\mathrm{C} 11-\mathrm{H} 11 \mathrm{~A}$ & 121.0 \\
\hline C10-C11-H11A & 121.0 \\
\hline C11-C12-C13 & $122.22(12)$ \\
\hline $\mathrm{C} 11-\mathrm{C} 12-\mathrm{Br} 1$ & $118.84(10)$ \\
\hline $\mathrm{C} 13-\mathrm{C} 12-\mathrm{Br} 1$ & $118.94(10)$ \\
\hline C12-C13-C14 & $118.72(12)$ \\
\hline C12-C13-H13A & 120.6 \\
\hline C14-C13-H13A & 120.6 \\
\hline $\mathrm{O} 2-\mathrm{C} 14-\mathrm{C} 13$ & $115.51(11)$ \\
\hline O2-C14-C9 & $123.91(11)$ \\
\hline C13-C14-C9 & $120.54(12)$ \\
\hline
\end{tabular}


Table S4. Anisotropic displacement parameters $\left(\AA^{2} \times 10^{3}\right)$ for zhu3. The anisotropic displacement factor exponent takes the form: $-2 \pi^{2}\left[h^{2} a^{* 2} U^{11}+\ldots+2 h k a^{*} b^{*} U^{12}\right]$

\begin{tabular}{lllllll}
\hline & $\mathrm{U}^{11}$ & $\mathrm{U}^{22}$ & $\mathrm{U}^{33}$ & $\mathrm{U}^{23}$ & $\mathrm{U}^{13}$ & $\mathrm{U}^{12}$ \\
\hline $\mathrm{Br} 1$ & $32(1)$ & $20(1)$ & $29(1)$ & $10(1)$ & $8(1)$ & $2(1)$ \\
$\mathrm{O} 1$ & $23(1)$ & $13(1)$ & $14(1)$ & $-1(1)$ & $1(1)$ & $4(1)$ \\
$\mathrm{O} 2$ & $16(1)$ & $15(1)$ & $16(1)$ & $3(1)$ & $4(1)$ & $4(1)$ \\
$\mathrm{C} 1$ & $18(1)$ & $19(1)$ & $15(1)$ & $-2(1)$ & $-1(1)$ & $0(1)$ \\
$\mathrm{C} 2$ & $15(1)$ & $22(1)$ & $19(1)$ & $4(1)$ & $4(1)$ & $2(1)$ \\
$\mathrm{C} 3$ & $14(1)$ & $14(1)$ & $13(1)$ & $1(1)$ & $1(1)$ & $1(1)$ \\
$\mathrm{C} 4$ & $14(1)$ & $13(1)$ & $13(1)$ & $0(1)$ & $-1(1)$ & $0(1)$ \\
$\mathrm{C} 5$ & $20(1)$ & $14(1)$ & $18(1)$ & $3(1)$ & $1(1)$ & $-1(1)$ \\
$\mathrm{C} 6$ & $25(1)$ & $13(1)$ & $21(1)$ & $1(1)$ & $-4(1)$ & $-1(1)$ \\
$\mathrm{C} 7$ & $24(1)$ & $13(1)$ & $19(1)$ & $-1(1)$ & $-1(1)$ & $5(1)$ \\
$\mathrm{C} 8$ & $15(1)$ & $13(1)$ & $12(1)$ & $-1(1)$ & $-1(1)$ & $2(1)$ \\
$\mathrm{C} 9$ & $14(1)$ & $13(1)$ & $12(1)$ & $-1(1)$ & $-2(1)$ & $0(1)$ \\
$\mathrm{C} 10$ & $16(1)$ & $17(1)$ & $16(1)$ & $-2(1)$ & $2(1)$ & $-1(1)$ \\
$\mathrm{C} 11$ & $20(1)$ & $19(1)$ & $16(1)$ & $1(1)$ & $4(1)$ & $-1(1)$ \\
$\mathrm{C} 12$ & $20(1)$ & $14(1)$ & $17(1)$ & $3(1)$ & $0(1)$ & $-2(1)$ \\
C13 & $16(1)$ & $14(1)$ & $18(1)$ & $1(1)$ & $0(1)$ & $1(1)$ \\
$\mathrm{C} 14$ & $13(1)$ & $14(1)$ & $12(1)$ & $0(1)$ & $0(1)$ & $0(1)$ \\
& & & & & & \\
\hline
\end{tabular}


Table S5. Hydrogen coordinates ( x $\left.10^{4}\right)$ and isotropic displacement parameters $\left(\AA^{2} \times 10^{3}\right)$ for zhu3.

\begin{tabular}{|c|c|c|c|c|}
\hline & $\mathrm{x}$ & $\mathrm{y}$ & $\mathrm{z}$ & $\mathrm{U}(\mathrm{eq})$ \\
\hline $\mathrm{H} 1 \mathrm{~A}$ & 2605 & 2510 & 282 & 26 \\
\hline H1B & 2144 & 3808 & 187 & 26 \\
\hline $\mathrm{H} 1 \mathrm{C}$ & 1321 & 2991 & 1043 & 26 \\
\hline $\mathrm{H} 2 \mathrm{~A}$ & 5531 & 3793 & 1720 & 28 \\
\hline $\mathrm{H} 2 \mathrm{~B}$ & 4864 & 4133 & 530 & 28 \\
\hline $\mathrm{H} 2 \mathrm{C}$ & 5154 & 2834 & 822 & 28 \\
\hline $\mathrm{H} 4 \mathrm{~A}$ & 3650 & 4587 & 2910 & 16 \\
\hline $\mathrm{H} 5 \mathrm{~A}$ & 2272 & 5583 & 997 & 21 \\
\hline H5B & 3928 & 5779 & 1350 & 21 \\
\hline H6A & 3237 & 6703 & 3000 & 24 \\
\hline H6B & 2368 & 7288 & 1994 & 24 \\
\hline H7A & 812 & 6906 & 3537 & 23 \\
\hline H7B & 281 & 6257 & 2434 & 23 \\
\hline H8A & 696 & 4342 & 2225 & 16 \\
\hline $\mathrm{H} 10 \mathrm{~A}$ & -299 & 3608 & 4462 & 19 \\
\hline H11A & -276 & 1926 & 5489 & 22 \\
\hline H13A & 3104 & 815 & 3610 & 19 \\
\hline
\end{tabular}




\section{Determination of $\mathrm{p} K_{\mathrm{a}}$ Values in $\mathrm{MeCN}$}

The $\mathrm{p} K_{\mathrm{a}}$ values for the compounds $\mathbf{4 b}, \mathbf{4} \mathbf{c}$ and $\mathbf{1 6}$ were measured using the UV-Vis spectrophotometric titration method described previously. ${ }^{9}$ The acidities of the compounds $4 \mathbf{4 a},{ }^{10} \mathbf{1 4},{ }^{10} \mathbf{1 5},{ }^{10}$ (S)-TRIP $(\mathbf{5}),{ }^{11}$ and $\mathrm{CF}_{3} \mathrm{COOH}^{12}$ in $\mathrm{MeCN}$ have been published earlier.

The essence of the method is determining relative acidities, i.e. $\Delta \mathrm{p} K_{\mathrm{a}}$ values. In a titration experiment a MeCN solutions of mixture of two acids - catalyst and reference acid - (both acids dissolved in the same solution) were titrated and UV-Vis spectra were collected. The solutions of the corresponding catalyst and reference acid were also titrated separately. The solutions of trifluoromethanesulfonic acid (Aldrich, 99+ \%) and tert-butyliminotris(pyrrolidino)phosphorane (Aldrich, $\geq 97 \%$ ) were used as acidic and basic titrant respectively. From the UV-Vis spectra dissociation degrees of both acids were obtained from every point of titration of the mixture. From the determined dissociation degree values of both acids in the same solution obtained, the $\mathrm{p} K_{\mathrm{a}}$ differences $\left(\Delta \mathrm{p} K_{\mathrm{a}}\right)$ were calculated. Every catalyst was measured against at least two reference acids. And the assigned $\mathrm{p} K_{\mathrm{a}}$ was found as average of $\mathrm{p} K_{\mathrm{a}}$ values obtained against different reference acids.

The experiments were conducted in an argon filled glovebox using acetonitrile (Romil $190 \mathrm{SpS}$ far UV/gradient quality) additionally dried by keeping on $3 \AA$ molecular sieves, leading to water content below $5 \mathrm{ppm}$. The concentrations of the test compounds and reference acids were kept below $10^{-4} \mathrm{M}$ when the dissociation constants were measured.

Table S6. The results of the determination of $\mathrm{p} K_{\mathrm{a}}$ values in acetonitrile.

\begin{tabular}{|c|c|c|c|c|c|}
\hline Compound & Reference acid (Ref) & $\mathrm{p} K_{\mathrm{a}}(\operatorname{Ref})^{\mathrm{a}}$ & $\Delta \mathrm{p} K_{\mathrm{a}}$ & $\mathrm{p} K_{\mathrm{a}}(\mathrm{Cat})$ & $\begin{array}{l}\text { Assigned } \\
\mathrm{pK}_{\mathrm{a}} \text { (Cat) }\end{array}$ \\
\hline \multirow{3}{*}{$4 b$} & Picric acid & 11.00 & -2.56 & 13.56 & \multirow{3}{*}{13.55} \\
\hline & $4-\mathrm{NO}_{2}-\mathrm{C}_{6} \mathrm{H}_{4}-\mathrm{CH}(\mathrm{CN})_{2}$ & 11.61 & -1.91 & 13.52 & \\
\hline & $\mathrm{C}_{6} \mathrm{~F}_{5}-\mathrm{CH}(\mathrm{CN})_{2}$ & 13.01 & -0.56 & 13.57 & \\
\hline \multirow{3}{*}{$4 c$} & Picric acid & 11.00 & -0.77 & 11.77 & \multirow{3}{*}{11.80} \\
\hline & $4-\mathrm{NO}_{2}-\mathrm{C}_{6} \mathrm{H}_{4}-\mathrm{CH}(\mathrm{CN})_{2}$ & 11.61 & -0.23 & 11.84 & \\
\hline & $\mathrm{C}_{6} \mathrm{~F}_{5}-\mathrm{CH}(\mathrm{CN})_{2}$ & 13.01 & 1.22 & 11.79 & \\
\hline \multirow{2}{*}{16} & $\mathrm{C}_{6} \mathrm{Br}_{5} \mathrm{OH}$ & 17.83 & 0.61 & 17.22 & \multirow{2}{*}{17.20} \\
\hline & $\begin{array}{c}(4-\mathrm{Cl}- \\
\left.\mathrm{C}_{6} \mathrm{~F}_{4}\right) \mathrm{CH}(\mathrm{CN}) \mathrm{COOEt}\end{array}$ & 17.39 & 0.20 & 17.19 & \\
\hline
\end{tabular}

${ }^{\text {a }}$ Values from ref. 9. 


\section{References}

1) a) An Efficient and Versatile Procedure for the Synthesis of Acetals from Aldehydes and Ketones Catalyzed by Lithium Tetrafluoroborate. Hamada, N.; Kazahaya, K.; Shimizu, H.; Sato, T. Synlett 2004, 1074; b) Lewis Acid Catalyzed Intramolecular Condensation of Ynol Ether-Acetals. Synthesis of Alkoxycycloalkene Carboxylates. Tran, V.; Minehan, T. G. Org. Lett. 2012, 14, 6100.

2) Intramolecular hydroalkoxylation catalyzed inside a self-assembled cavity of an enzyme-like host structure. Catti, L.; Tiefenbacher, K. Chem. Commun. 2015, 51, 892.

3) Utimoto, K.; Sakai, N.; Obaya-shi, M.; Nozaki, H. Tetrahedron 1976, 32, 769 and followed by hydrolysis and reduction of the nitrile precursor.

4) One-Pot Regio- and Stereoselective Cyclization of 1,2,n-Triols. Zheng, T.; Narayan, R. S.; Schomaker, J. M.; Borhan, B. J. Am. Chem. Soc. 2005, 127, 6946.

5) Stereoselective Synthesis of Pyrano[3,2-c]benzopyrans via Intramolecular Cycloaddition of o-Quinonemethides Generated from Salicylaldehydes and Unsaturated Alcohols under Very Mild Conditions. Miyazaki, H.; Honda, K.; Asami, M.; Inoue, S. J. Org. Chem. 1999, 64, 9507.

6) Diastereoselective synthesis of substituted hexahydrobenzo[de]isochromanes and evaluation of their antileishmanial activity. Saikia, A. K.; Sultana, S.; Devi, N. R.; Deka, M. J.; Tiwari, K.; Dubey, V. K. Org. Biomol. Chem. 2016, 14, 970.

7) Diastereoselective Synthesis of Angularly Fused Pyranochromenes. Sarmah, B.; Baishya, G.; Hazarika, N.; Das, P. J. Synlett 2015, 26, 2151.

8) Catalytic Asymmetric Intramolecular [4+2] Cycloaddition of In Situ Generated ortho-Quinone Methides. Xie, Y.; List, B. Angew. Chem. Int. Ed. 2017, 56, 4936.

9) A Comprehensive Self-Consistent Spectrophotometric Acidity Scale of Neutral Brønsted Acids in Acetonitrile. Kütt, A.; Leito, I.; Kaljurand, I.; Sooväli, L.; Vlasov, V. M.; Yagupolskii, L. M.; Koppel, I. A. J. Org. Chem. 2006, $71,2829$.

10) Highly Acidic Conjugate-Base-Stabilized Carboxylic Acids Catalyze Enantioselective oxa-Pictet-Spengler Reactions with Ketals. Zhu, Z.; Odagi, M.; Zhao, C.; Abboud, K. A.; Kirm, H. U.; Saame, J.; Lõkov, M.; Leito, I.; Seidel, D. Angew. Chem. Int. Ed. 2020, 59, 2028.

11) On the Acidity and Reactivity of Highly Effective Chiral Brønsted Acid Catalysts: Establishment of an Acidity Scale. Kaupmees, K.; Tolstoluzhsky, N.; Raja, S.; Rueping, M.; Leito, I. Angew. Chem. Int. Ed. 2013, 52, 11569.

12) Prediction of acidity in acetonitrile solution with COSMO-RS. Eckert, F.; Leito, I.; Kaljurand, I.; Kütt, A.; Klamt, A.; Diedenhofen, M. J. Comput. Chem. 2009, 30, 799. 


\section{SFC and HPLC Profiles}

\section{SFC Profile of 3a:}

Conditions: Daicel Chiralcel OJ-H, column temperature $=40{ }^{\circ} \mathrm{C}, \mathrm{CO}_{2} / \mathrm{MeOH}=95 / 5$, Flow rate $=2 \mathrm{~mL} / \mathrm{min}, \mathrm{UV}=$ $280 \mathrm{~nm}, \mathrm{t}_{\mathrm{R}}=3.7 \mathrm{~min}$ (major) and $\mathrm{t}_{\mathrm{R}}=5.6 \mathrm{~min}$ (minor).
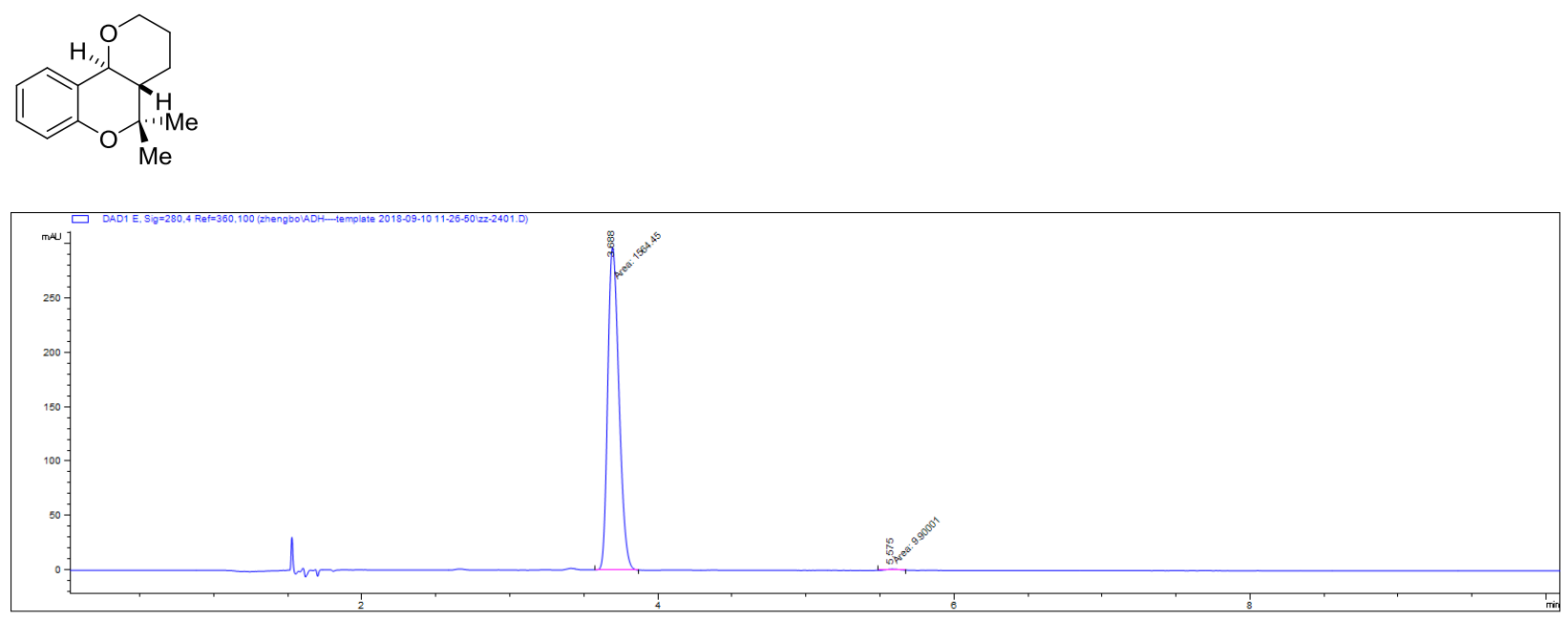

\begin{tabular}{|l|l|l|}
\hline Peak & Retention time (min) & Area \% \\
\hline 1 & 3.688 & 99.371 \\
\hline 2 & 5.575 & 0.629 \\
\hline
\end{tabular}

Racemic sample

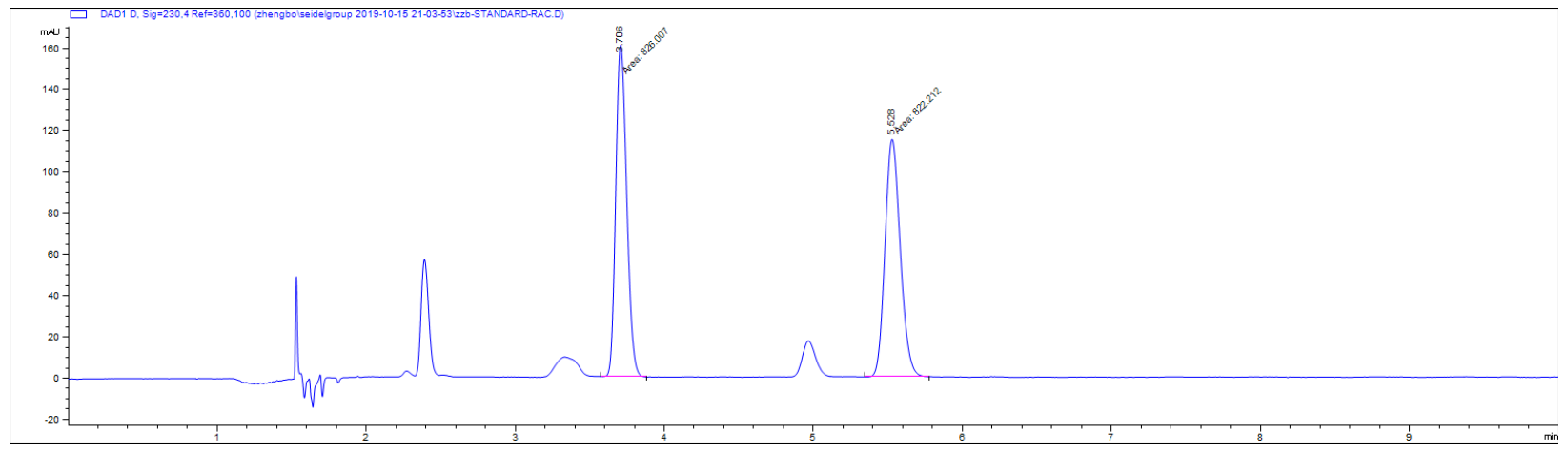

\begin{tabular}{|l|l|l|}
\hline Peak & Retention time (min) & Area \% \\
\hline 1 & 3.706 & 50.085 \\
\hline 2 & 5.528 & 49.915 \\
\hline
\end{tabular}




\section{SFC Profile of 3b:}

Conditions: Daicel Chiralcel AD-H, column temperature $=40{ }^{\circ} \mathrm{C}, \mathrm{CO}_{2} / \mathrm{MeOH}=90 / 10$, Flow rate $=2 \mathrm{~mL} / \mathrm{min}$, UV $=230 \mathrm{~nm}, \mathrm{t}_{\mathrm{R}}=3.2 \mathrm{~min}$ (minor) and $\mathrm{t}_{\mathrm{R}}=3.5 \min$ (major).
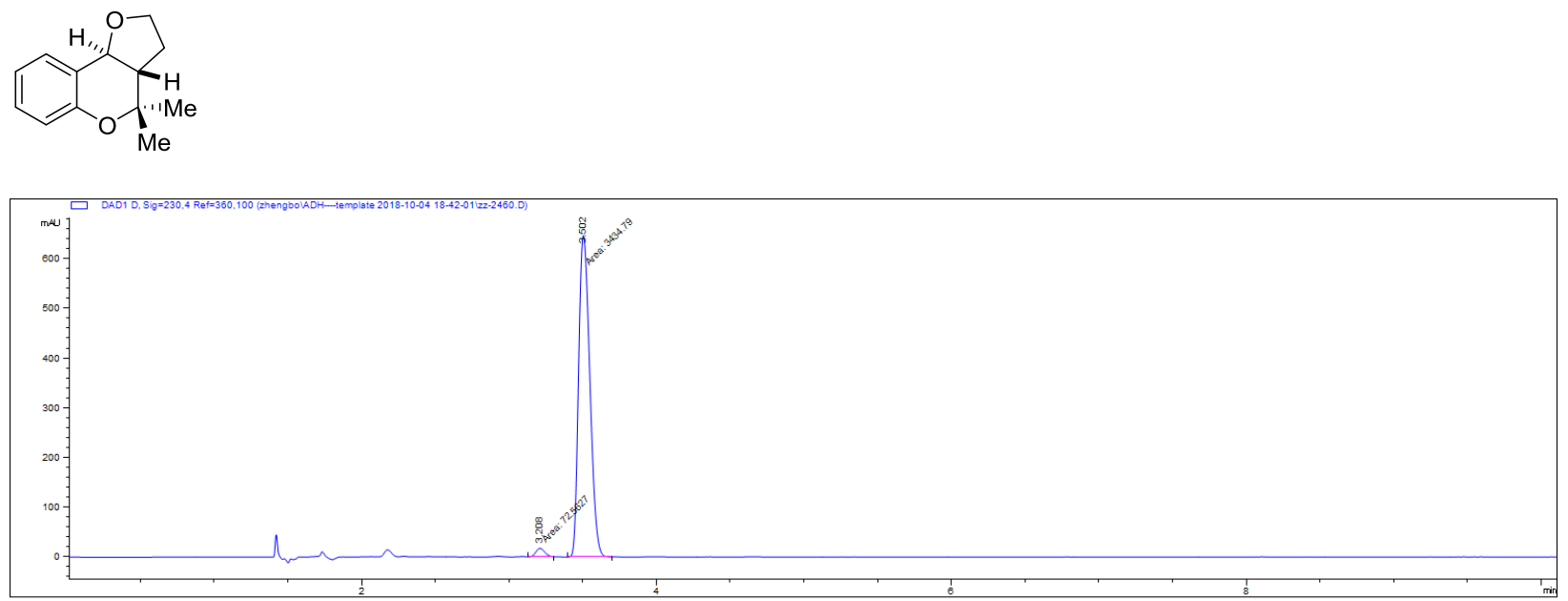

\begin{tabular}{|l|l|l|}
\hline Peak & Retention time (min) & Area \% \\
\hline 1 & 3.208 & 2.069 \\
\hline 2 & 3.502 & 97.931 \\
\hline
\end{tabular}

\section{Racemic sample}

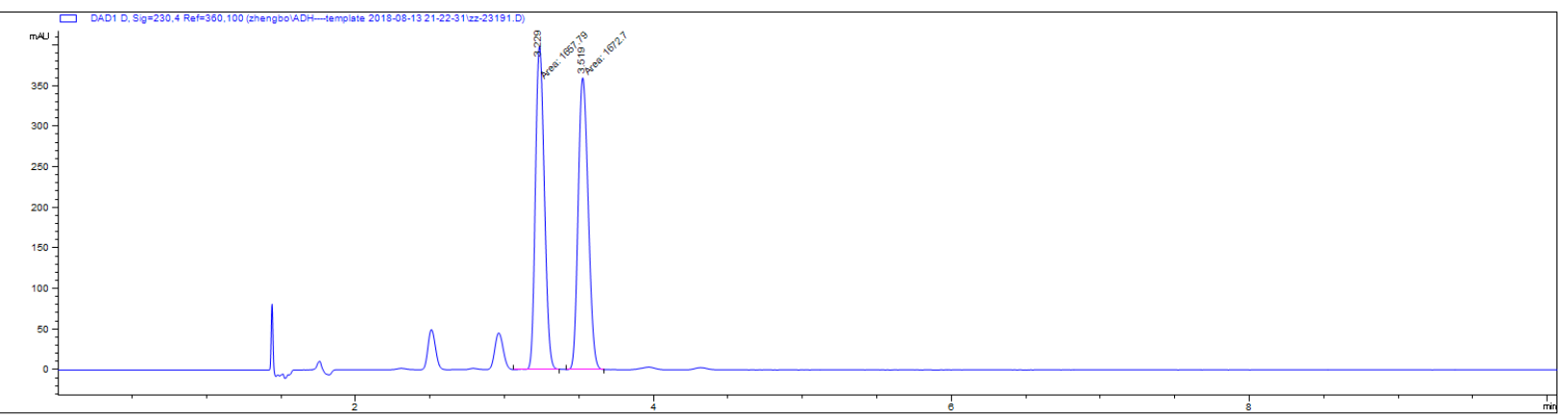

\begin{tabular}{|l|l|l|}
\hline Peak & Retention time (min) & Area \% \\
\hline 1 & 3.229 & 49.776 \\
\hline 2 & 3.519 & 50.224 \\
\hline
\end{tabular}




\section{SFC Profile of 3c:}

Conditions: Daicel Chiralcel AY-H, column temperature $=40{ }^{\circ} \mathrm{C}, \mathrm{CO}_{2} / \mathrm{MeOH}=90 / 10$, Flow rate $=2 \mathrm{~mL} / \mathrm{min}$, UV $=280 \mathrm{~nm}, \mathrm{t}_{\mathrm{R}}=4.4 \min$ (major) and $\mathrm{t}_{\mathrm{R}}=4.8 \min$ (minor).
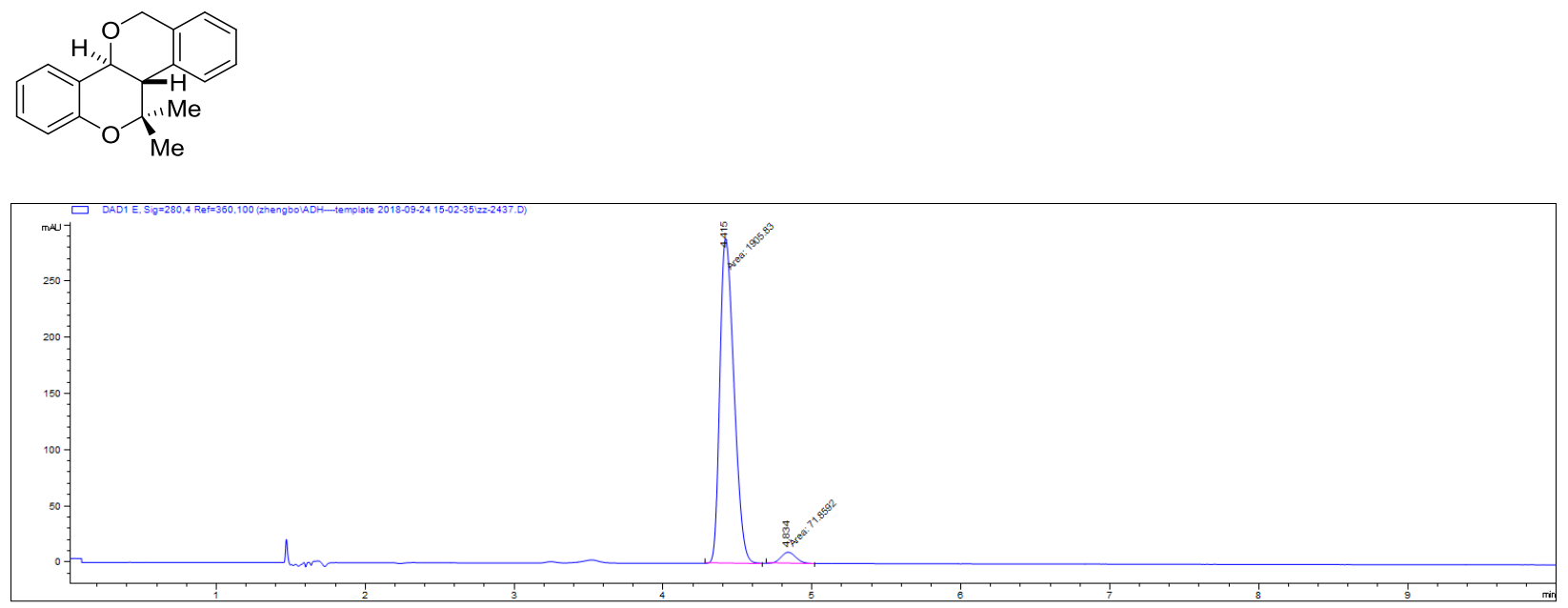

\begin{tabular}{|l|l|l|}
\hline Peak & Retention time (min) & Area \% \\
\hline 1 & 4.415 & 96.367 \\
\hline 2 & 4.834 & 3.633 \\
\hline
\end{tabular}

\section{Racemic sample}

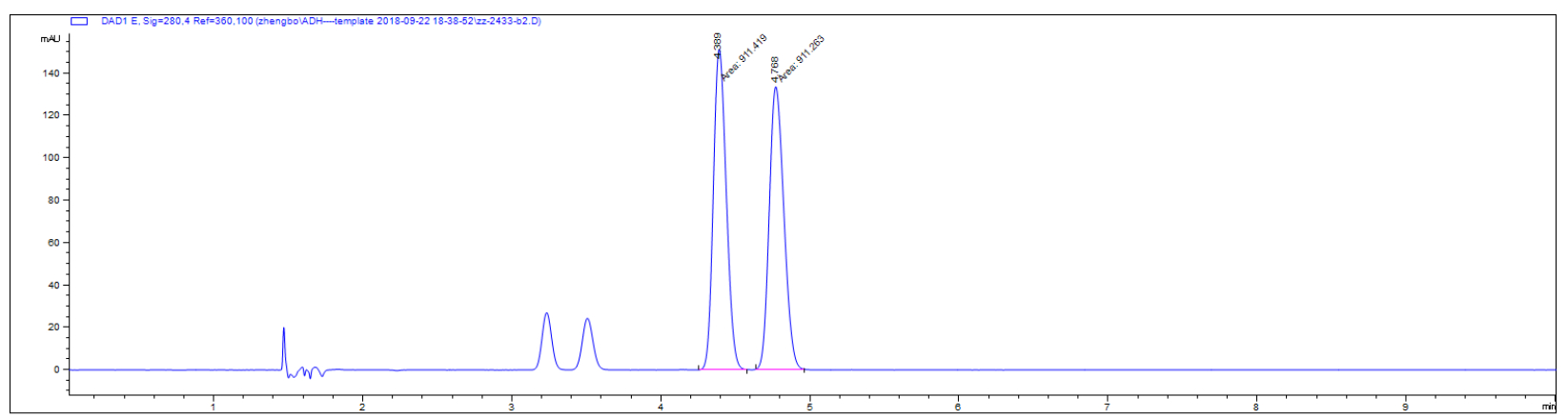

\begin{tabular}{|l|l|l|}
\hline Peak & Retention time (min) & Area \% \\
\hline 1 & 4.389 & 50.004 \\
\hline 2 & 4.768 & 49.996 \\
\hline
\end{tabular}




\section{SFC Profile of 3d:}

Conditions: Daicel Chiralcel AD-H, column temperature $=40{ }^{\circ} \mathrm{C}, \mathrm{CO}_{2} / \mathrm{MeOH}=80 / 20$, Flow rate $=2 \mathrm{~mL} / \mathrm{min}$, UV $=280 \mathrm{~nm}, \mathrm{t}_{\mathrm{R}}=2.6 \min \left(\right.$ minor) and $\mathrm{t}_{\mathrm{R}}=2.9 \min$ (major).
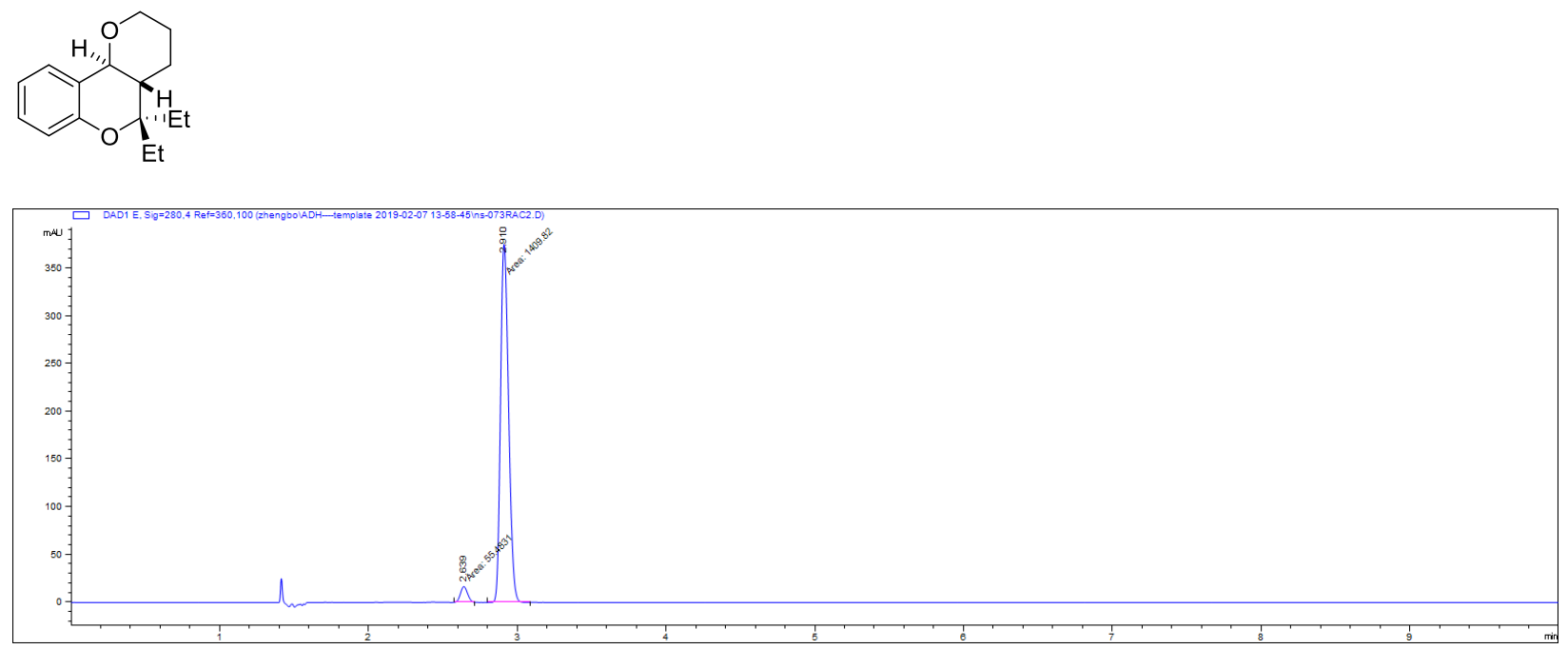

\begin{tabular}{|l|l|l|}
\hline Peak & Retention time (min) & Area \% \\
\hline 1 & 2.639 & 3.787 \\
\hline 2 & 2.910 & 96.213 \\
\hline
\end{tabular}

Racemic sample

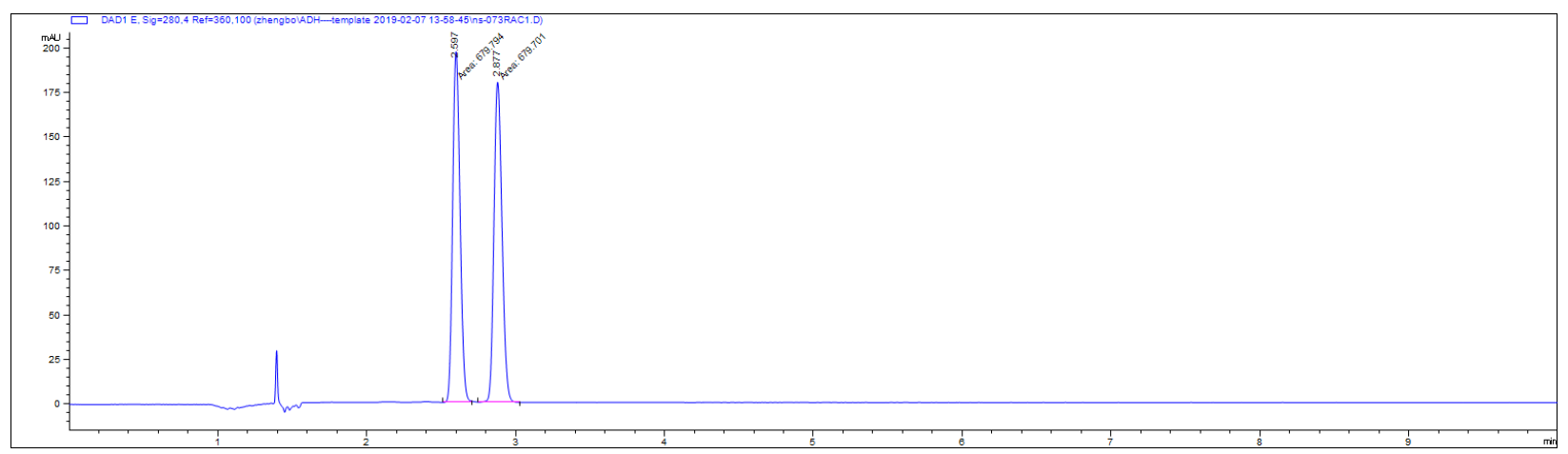

\begin{tabular}{|l|l|l|}
\hline Peak & Retention time (min) & Area \% \\
\hline 1 & 2.597 & 50.003 \\
\hline 2 & 2.877 & 49.997 \\
\hline
\end{tabular}




\section{SFC Profile of 3e:}

Conditions: Daicel Chiralcel OJ-H, column temperature $=40{ }^{\circ} \mathrm{C}, \mathrm{CO}_{2} / \mathrm{MeOH}=90 / 10$, Flow rate $=2 \mathrm{~mL} / \mathrm{min}, \mathrm{UV}=$ $280 \mathrm{~nm}, \mathrm{t}_{\mathrm{R}}=3.3 \mathrm{~min}$ (major) and $\mathrm{t}_{\mathrm{R}}=3.6 \mathrm{~min}$ (minor)
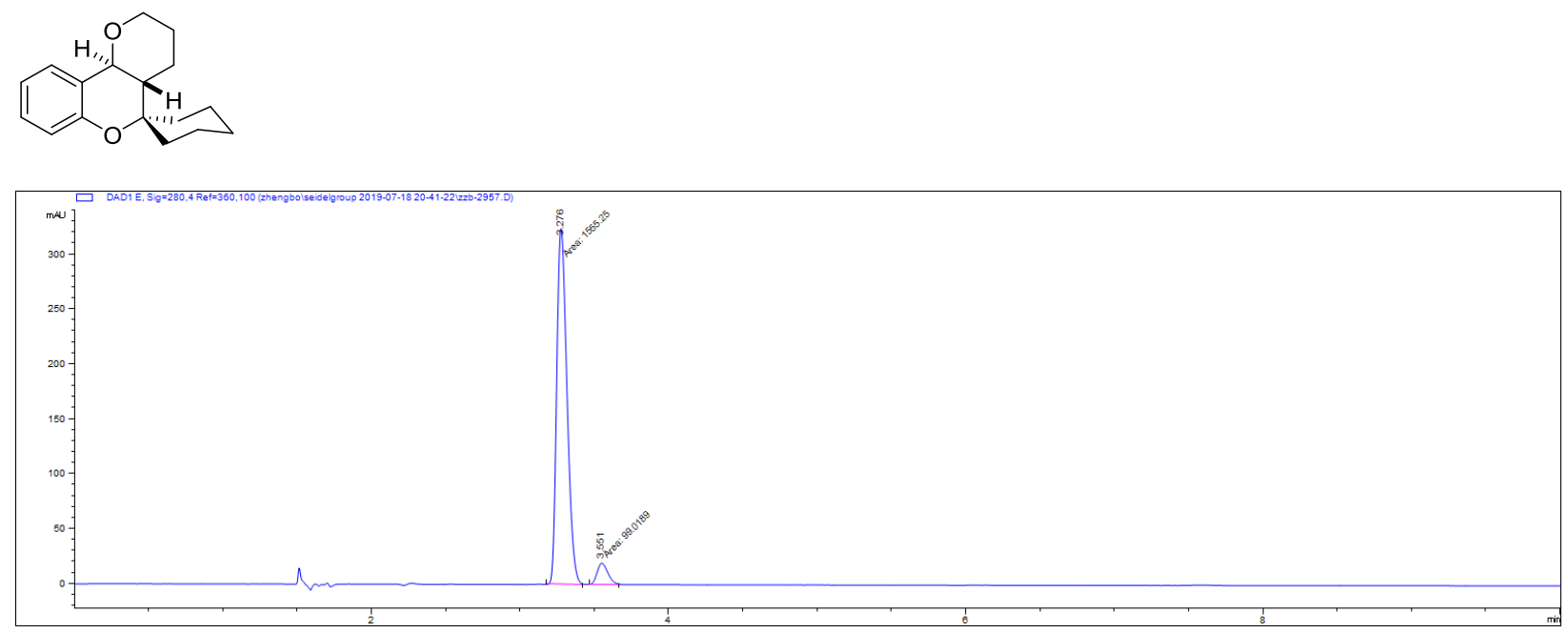

\begin{tabular}{|l|l|l|}
\hline Peak & Retention time (min) & Area \% \\
\hline 1 & 3.276 & 94.014 \\
\hline 2 & 3.551 & 5.986 \\
\hline
\end{tabular}

Racemic sample

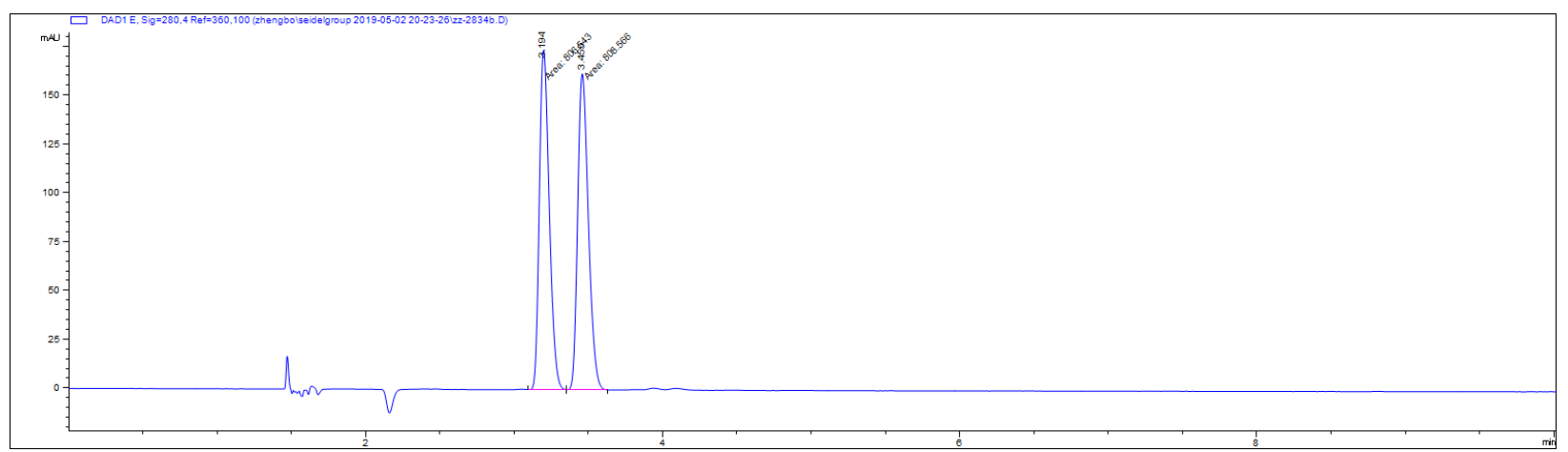

\begin{tabular}{|l|l|l|}
\hline Peak & Retention time (min) & Area \% \\
\hline 1 & 3.194 & 49.937 \\
\hline 2 & 3.455 & 50.063 \\
\hline
\end{tabular}


SFC Profile of 3f:

Conditions: Daicel Chiralcel OJ-H, column temperature $=40{ }^{\circ} \mathrm{C}, \mathrm{CO}_{2} / \mathrm{MeOH}=90 / 10$, Flow rate $=2 \mathrm{~mL} / \mathrm{min}, \mathrm{UV}=$ $230 \mathrm{~nm}, \mathrm{t}_{\mathrm{R}}=2.7 \min$ (minor) and $\mathrm{t}_{\mathrm{R}}=3.6 \min$ (major).
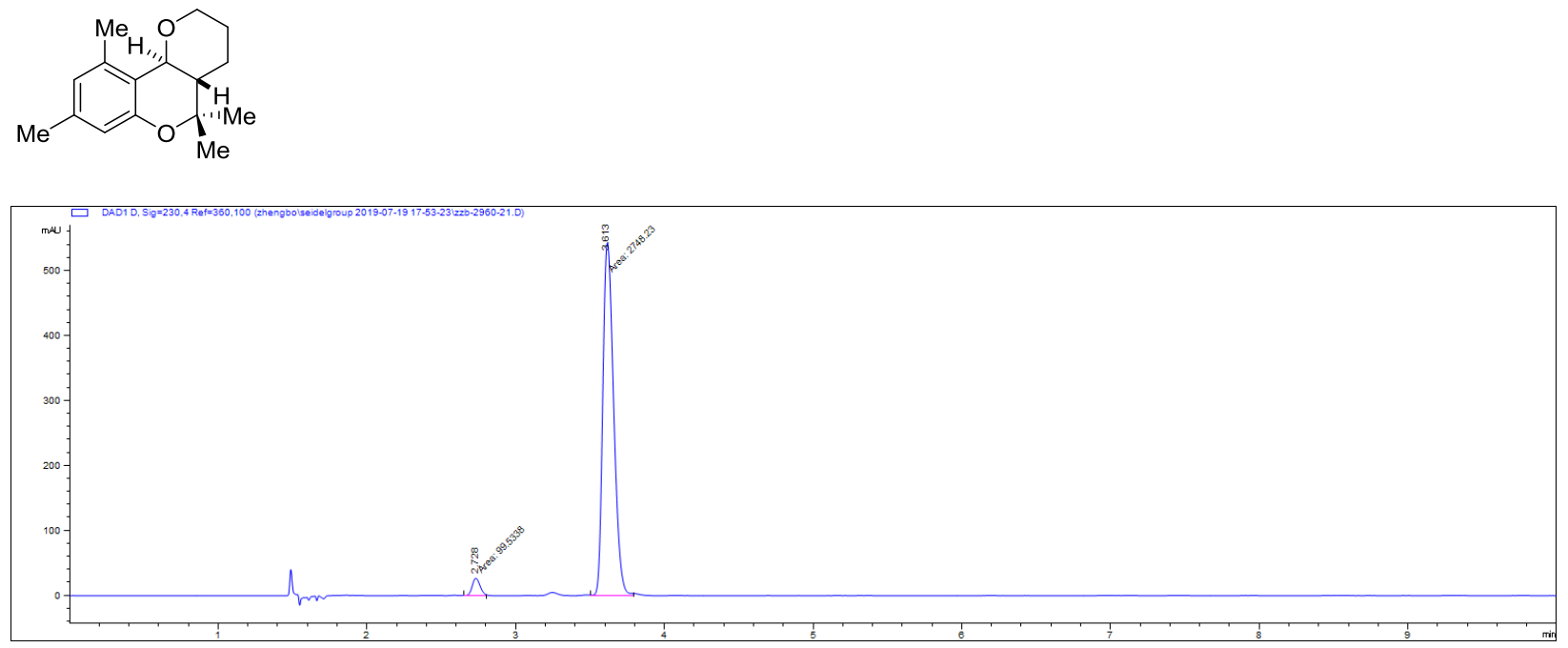

\begin{tabular}{|l|l|l|}
\hline Peak & Retention time (min) & Area \% \\
\hline 1 & 2.728 & 3.495 \\
\hline 2 & 3.613 & 96.505 \\
\hline
\end{tabular}

Racemic sample

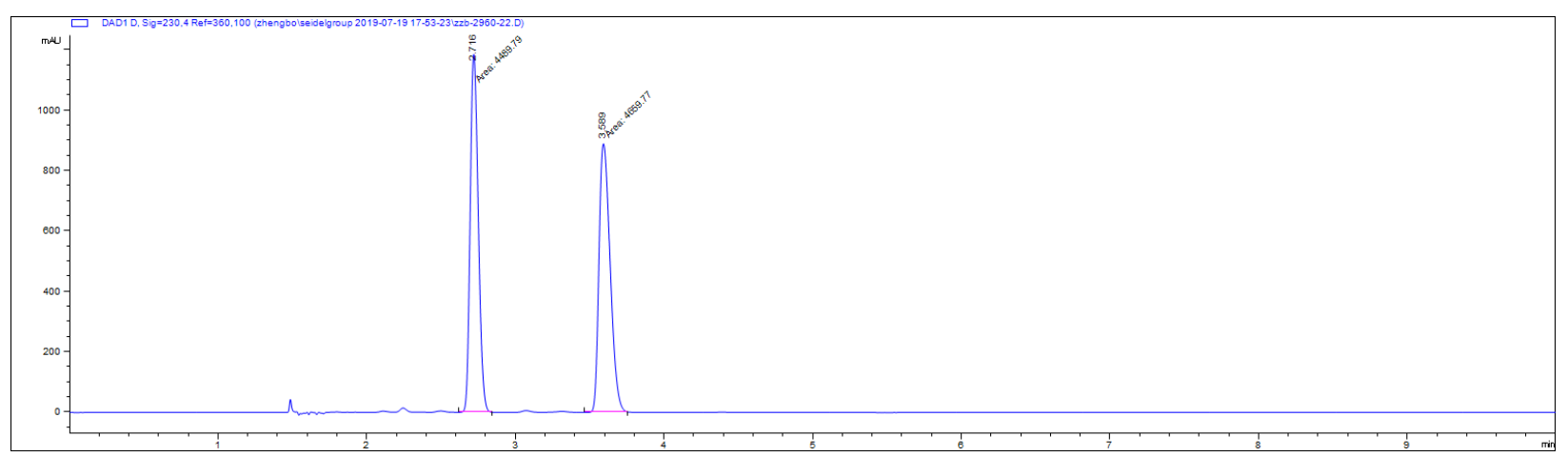

\begin{tabular}{|l|l|l|}
\hline Peak & Retention time (min) & Area \% \\
\hline 1 & 2.716 & 49.071 \\
\hline 2 & 3.589 & 50.929 \\
\hline
\end{tabular}




\section{SFC Profile of 3g:}

Conditions: Daicel Chiralcel OJ-H, column temperature $=40{ }^{\circ} \mathrm{C}, \mathrm{CO}_{2} / \mathrm{MeOH}=95 / 5$, Flow rate $=2 \mathrm{~mL} / \mathrm{min}, \mathrm{UV}=$ $280 \mathrm{~nm}, \mathrm{t}_{\mathrm{R}}=2.6 \mathrm{~min}$ (major) and $\mathrm{t}_{\mathrm{R}}=3.3 \mathrm{~min}$ (minor).
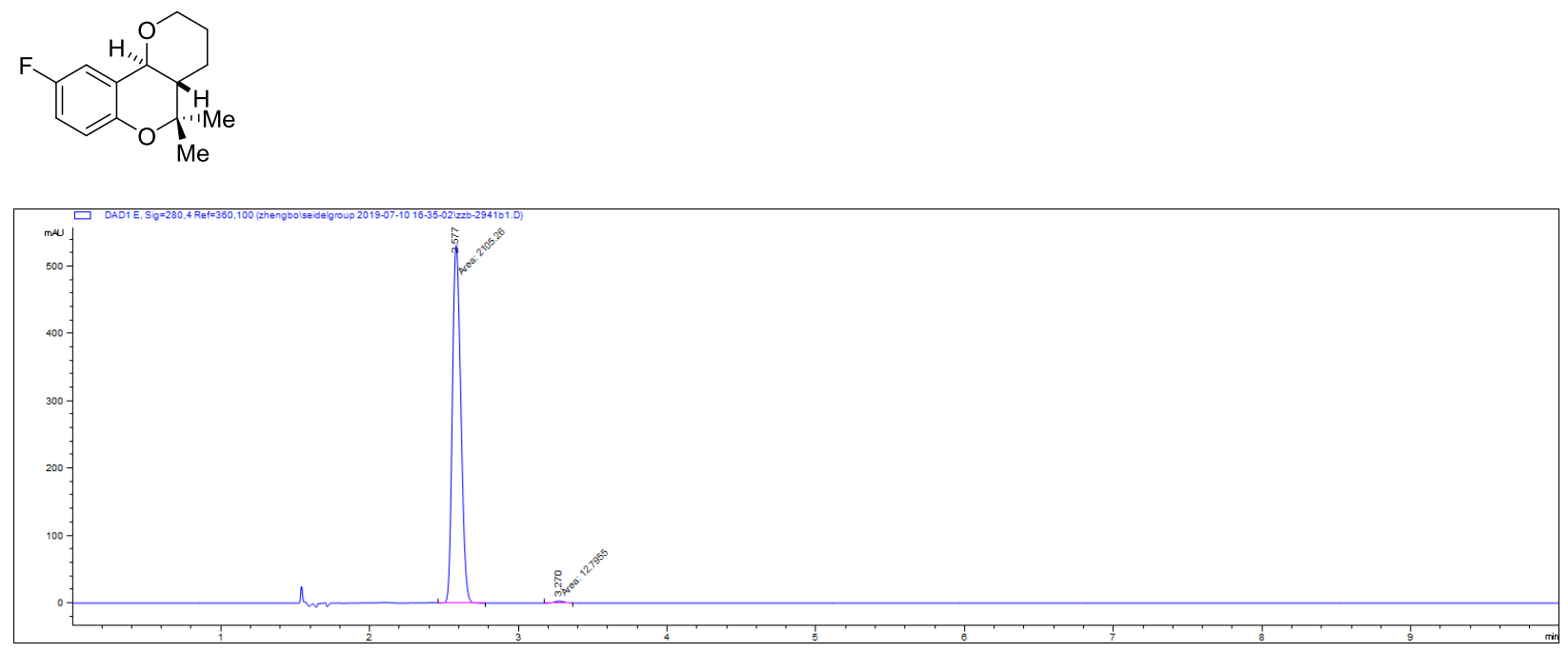

\begin{tabular}{|l|l|l|}
\hline Peak & Retention time (min) & Area \% \\
\hline 1 & 2.577 & 99.396 \\
\hline 2 & 3.270 & 0.604 \\
\hline
\end{tabular}

Racemic sample

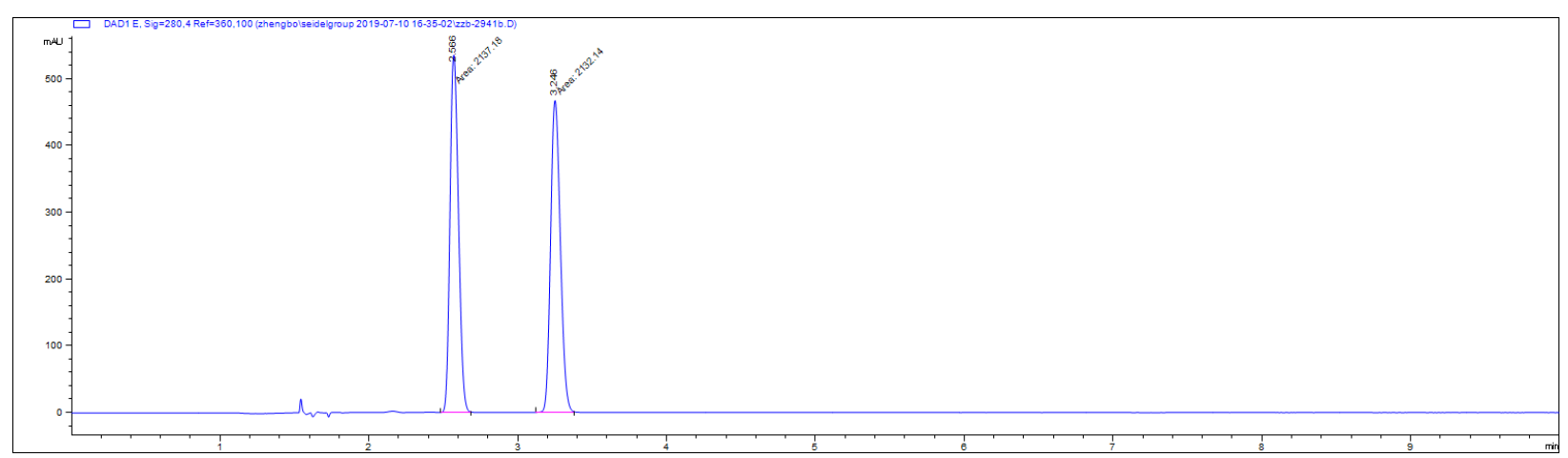

\begin{tabular}{|l|l|l|}
\hline Peak & Retention time (min) & Area \% \\
\hline 1 & 2.566 & 50.059 \\
\hline 2 & 3.246 & 49.941 \\
\hline
\end{tabular}




\section{SFC Profile of 3h:}

Conditions: Daicel Chiralcel OJ-H, column temperature $=40{ }^{\circ} \mathrm{C}, \mathrm{CO}_{2} / \mathrm{MeOH}=90 / 10$, Flow rate $=2 \mathrm{~mL} / \mathrm{min}, \mathrm{UV}=$ $280 \mathrm{~nm}, \mathrm{t}_{\mathrm{R}}=2.9 \min$ (major) and $\mathrm{t}_{\mathrm{R}}=3.4 \min$ (minor).
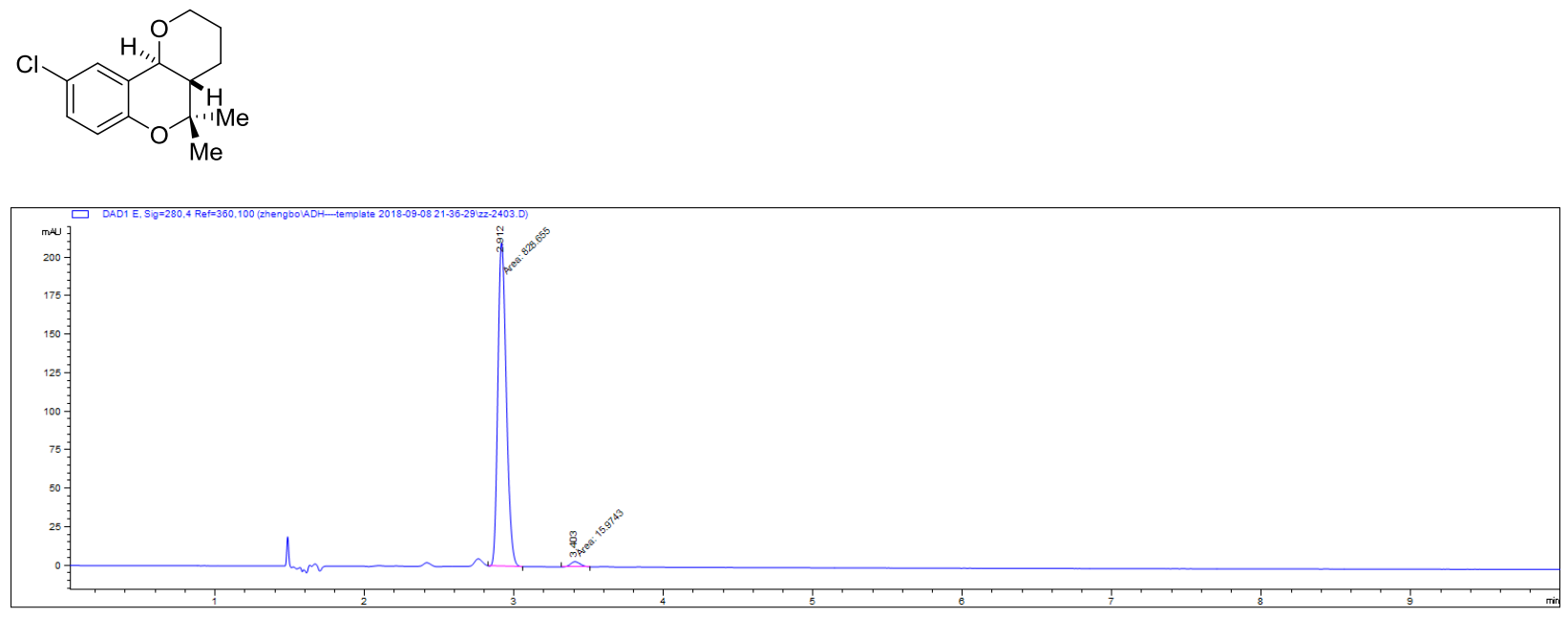

\begin{tabular}{|l|l|l|}
\hline Peak & Retention time (min) & Area \% \\
\hline 1 & 2.912 & 98.109 \\
\hline 2 & 3.403 & 1.891 \\
\hline
\end{tabular}

Racemic sample

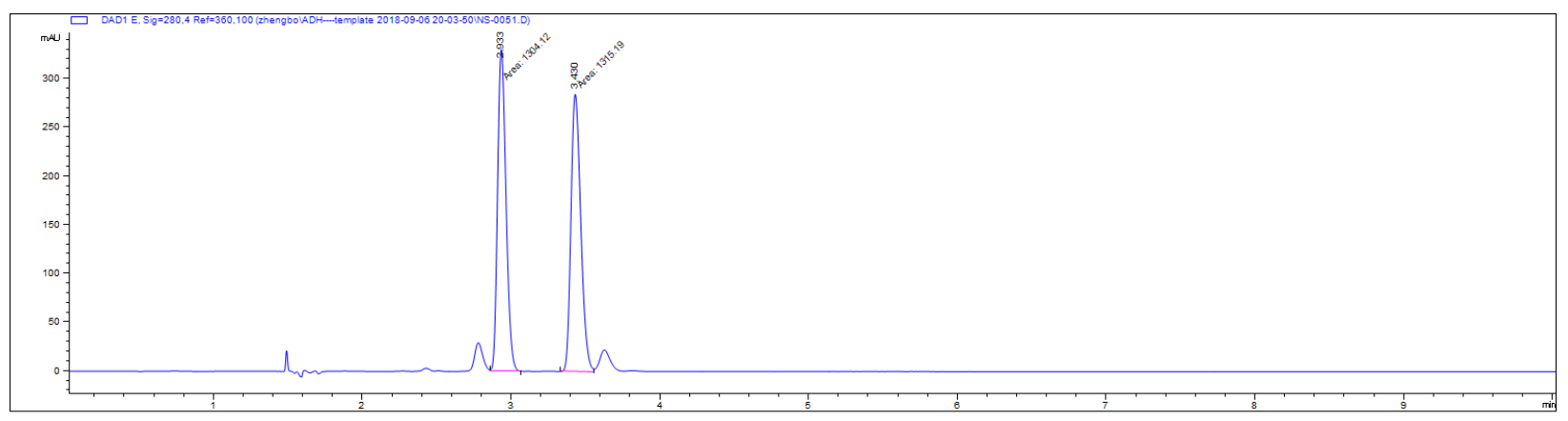

\begin{tabular}{|l|l|l|}
\hline Peak & Retention time (min) & Area \% \\
\hline 1 & 2.933 & 49.789 \\
\hline 2 & 3.430 & 50.211 \\
\hline
\end{tabular}




\section{SFC Profile of 3i:}

Conditions: Daicel Chiralcel OJ-H, column temperature $=40{ }^{\circ} \mathrm{C}, \mathrm{CO}_{2} / \mathrm{MeOH}=90 / 10$, Flow rate $=2 \mathrm{~mL} / \mathrm{min}, \mathrm{UV}=$ $230 \mathrm{~nm}, \mathrm{t}_{\mathrm{R}}=3.4 \min$ (major) and $\mathrm{t}_{\mathrm{R}}=3.8 \mathrm{~min}$ (minor).
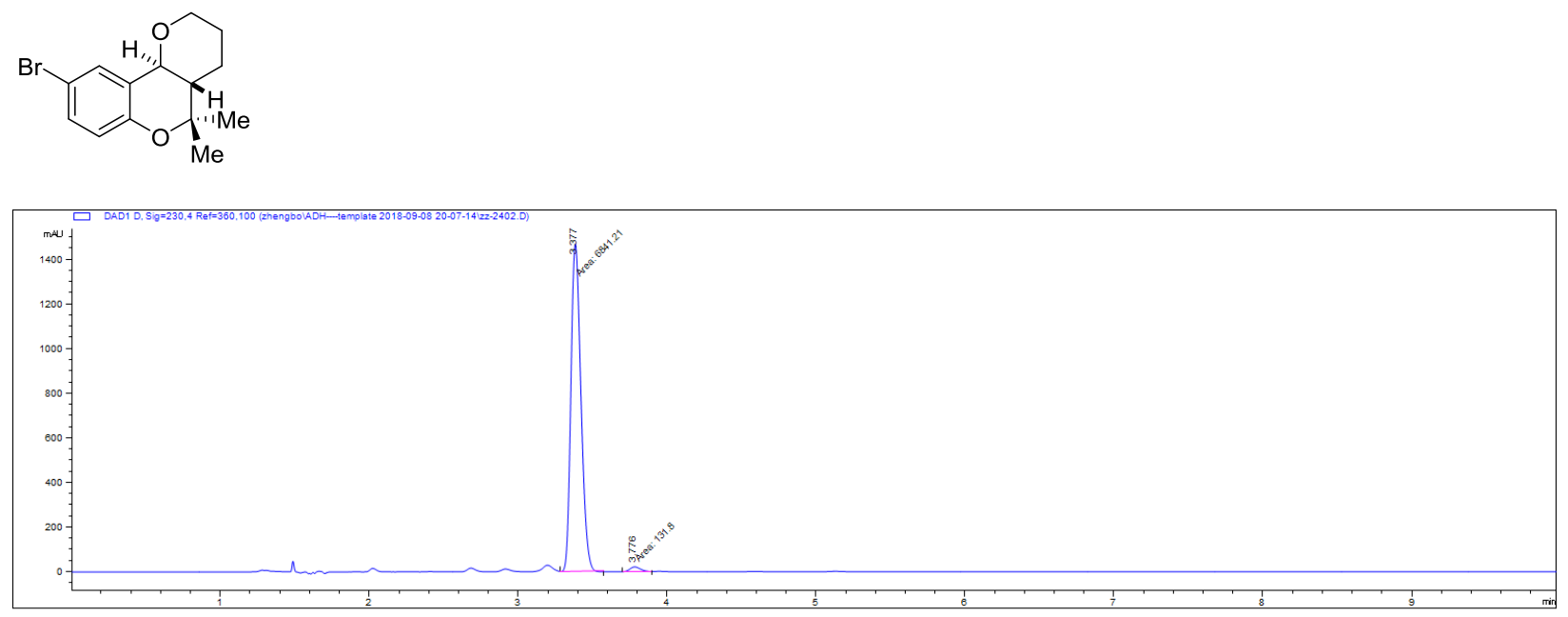

\begin{tabular}{|l|l|l|}
\hline Peak & Retention time (min) & Area \% \\
\hline 1 & 3.377 & 98.110 \\
\hline 2 & 3.776 & 1.890 \\
\hline
\end{tabular}

Racemic sample

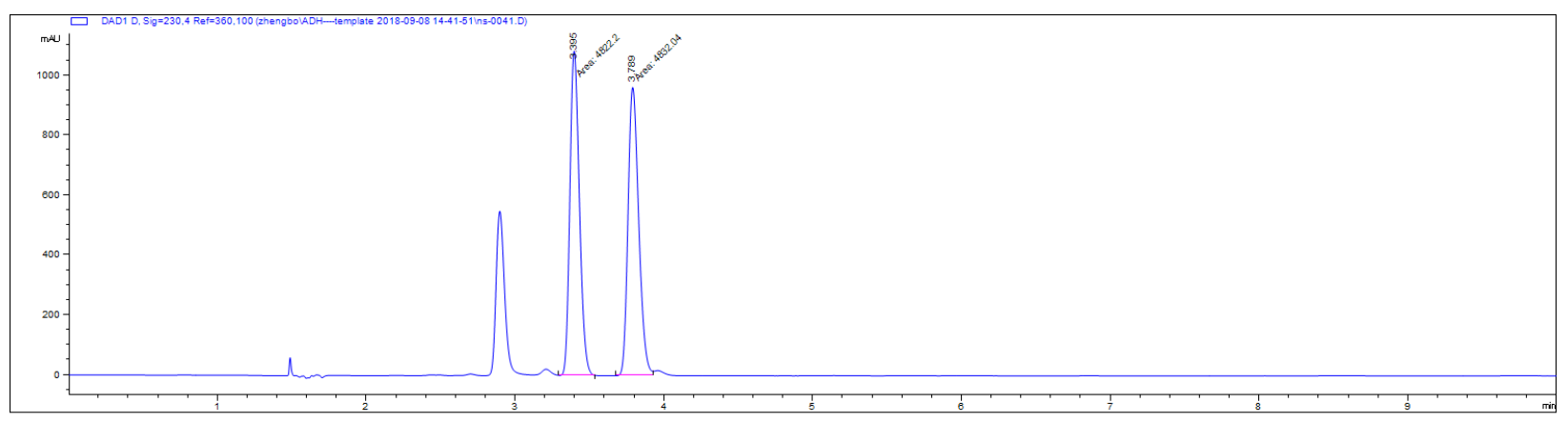

\begin{tabular}{|l|l|l|}
\hline Peak & Retention time (min) & Area \% \\
\hline 1 & 3.395 & 49.949 \\
\hline 2 & 3.789 & 50.051 \\
\hline
\end{tabular}


SFC Profile of $\mathbf{3 j}$ :

Conditions: Daicel Chiralcel OJ-H, column temperature $=40{ }^{\circ} \mathrm{C}, \mathrm{CO}_{2} / \mathrm{MeOH}=97 / 3$, Flow rate $=2 \mathrm{~mL} / \mathrm{min}, \mathrm{UV}=$ $280 \mathrm{~nm}, \mathrm{t}_{\mathrm{R}}=4.8 \mathrm{~min}$ (major) and $\mathrm{t}_{\mathrm{R}}=5.2 \mathrm{~min}$ (minor).
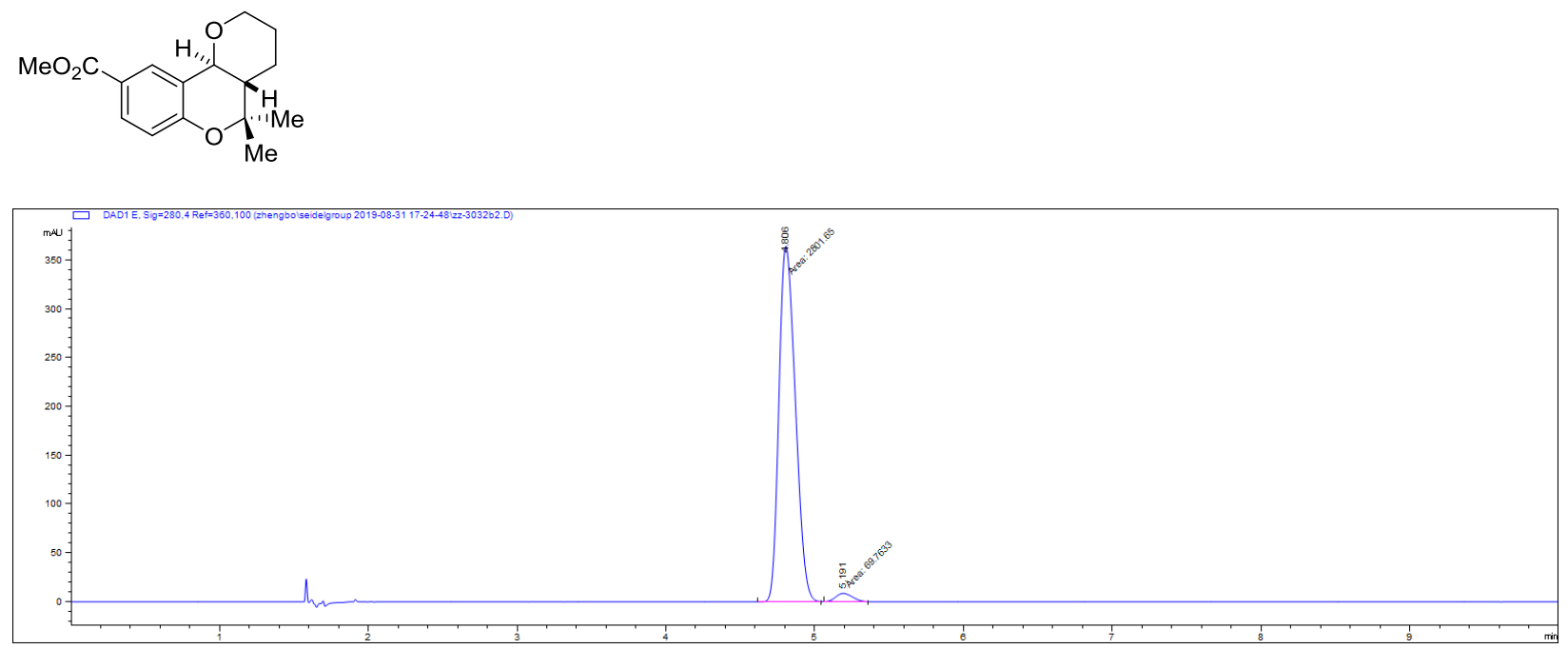

\begin{tabular}{|l|l|l|}
\hline Peak & Retention time (min) & Area \% \\
\hline 1 & 4.806 & 97.569 \\
\hline 2 & 5.191 & 2.431 \\
\hline
\end{tabular}

Racemic sample

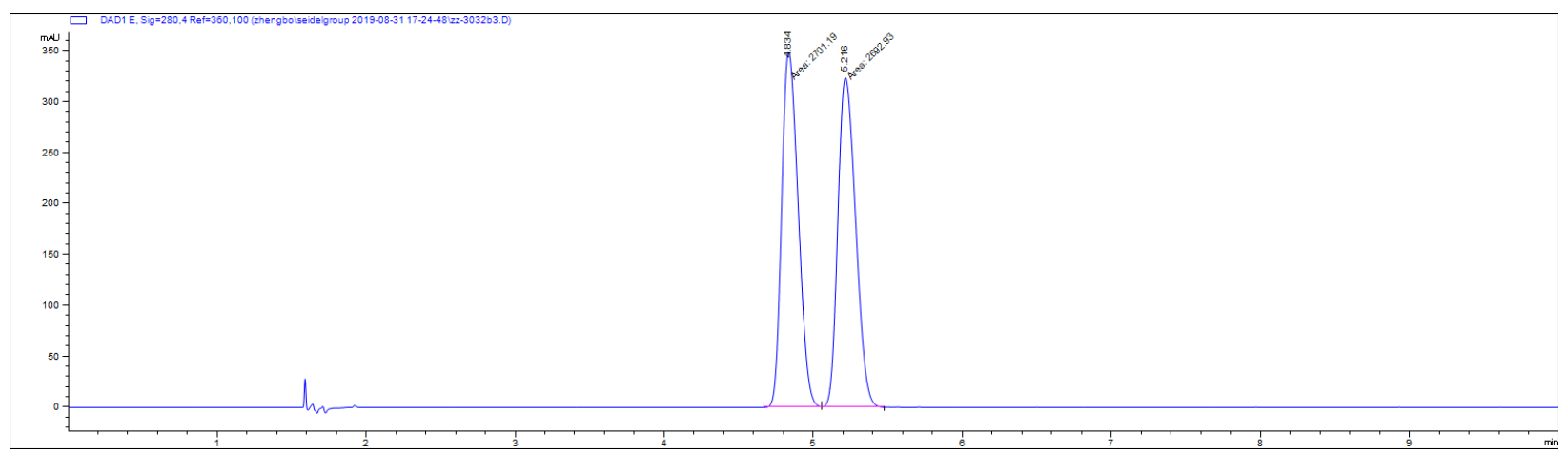

\begin{tabular}{|l|l|l|}
\hline Peak & Retention time (min) & Area \% \\
\hline 1 & 4.834 & 50.077 \\
\hline 2 & 5.216 & 49.923 \\
\hline
\end{tabular}




\section{SFC Profile of 3k:}

Conditions: Daicel Chiralcel OJ-H, column temperature $=40{ }^{\circ} \mathrm{C}, \mathrm{CO}_{2} / \mathrm{MeOH}=90 / 10$, Flow rate $=2 \mathrm{~mL} / \mathrm{min}, \mathrm{UV}=$ $230 \mathrm{~nm}, \mathrm{t}_{\mathrm{R}}=3.1 \mathrm{~min}$ (major) and $\mathrm{t}_{\mathrm{R}}=3.8 \mathrm{~min}$ (minor).
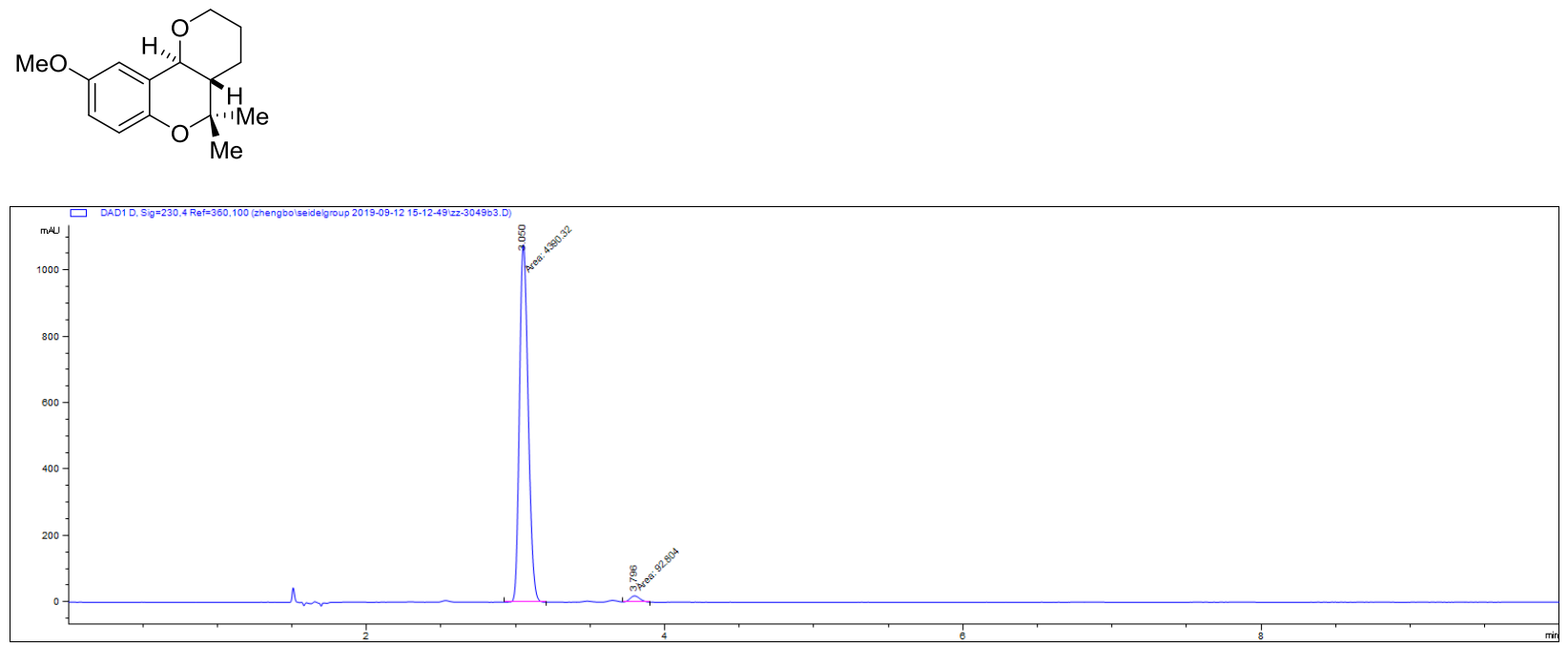

\begin{tabular}{|l|l|l|}
\hline Peak & Retention time (min) & Area \% \\
\hline 1 & 3.050 & 97.930 \\
\hline 2 & 3.796 & 2.070 \\
\hline
\end{tabular}

Racemic sample

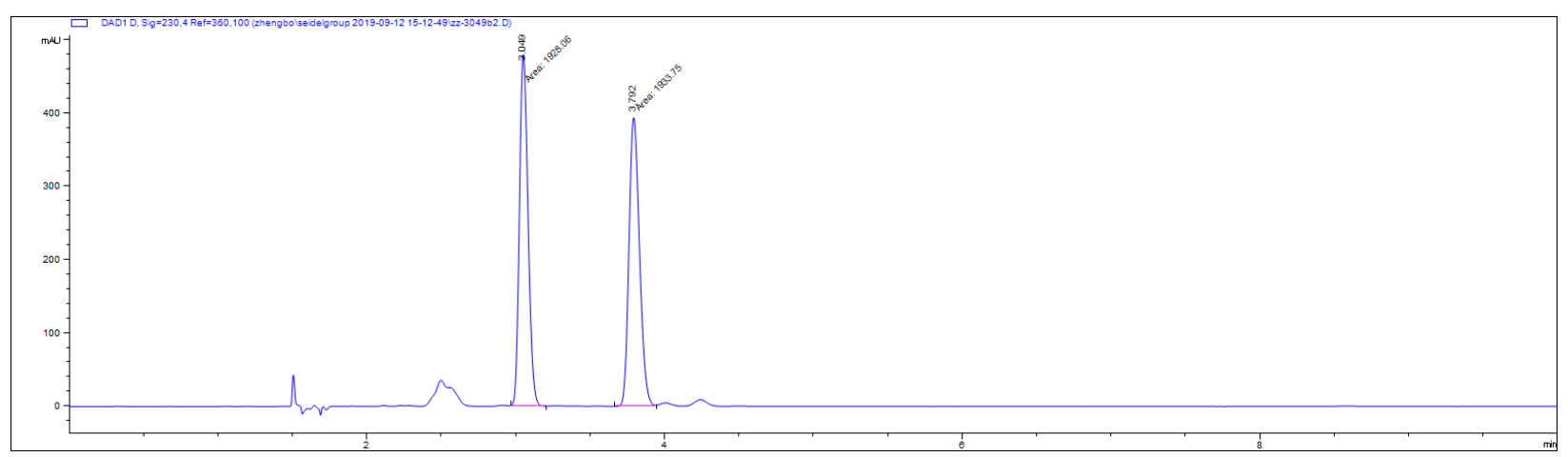

\begin{tabular}{|l|l|l|}
\hline Peak & Retention time (min) & Area \% \\
\hline 1 & 3.049 & 49.929 \\
\hline 2 & 3.792 & 50.071 \\
\hline
\end{tabular}


HPLC Profile of 31:

Conditions: Daicel Chiralpak OJ-H, $n$-hexane $/ i-\mathrm{PrOH}=90 / 10$, Flow rate $=1 \mathrm{~mL} / \mathrm{min}, \mathrm{UV}=280 \mathrm{~nm}, \mathrm{t}_{\mathrm{R}}=6.4 \mathrm{~min}$ (major).
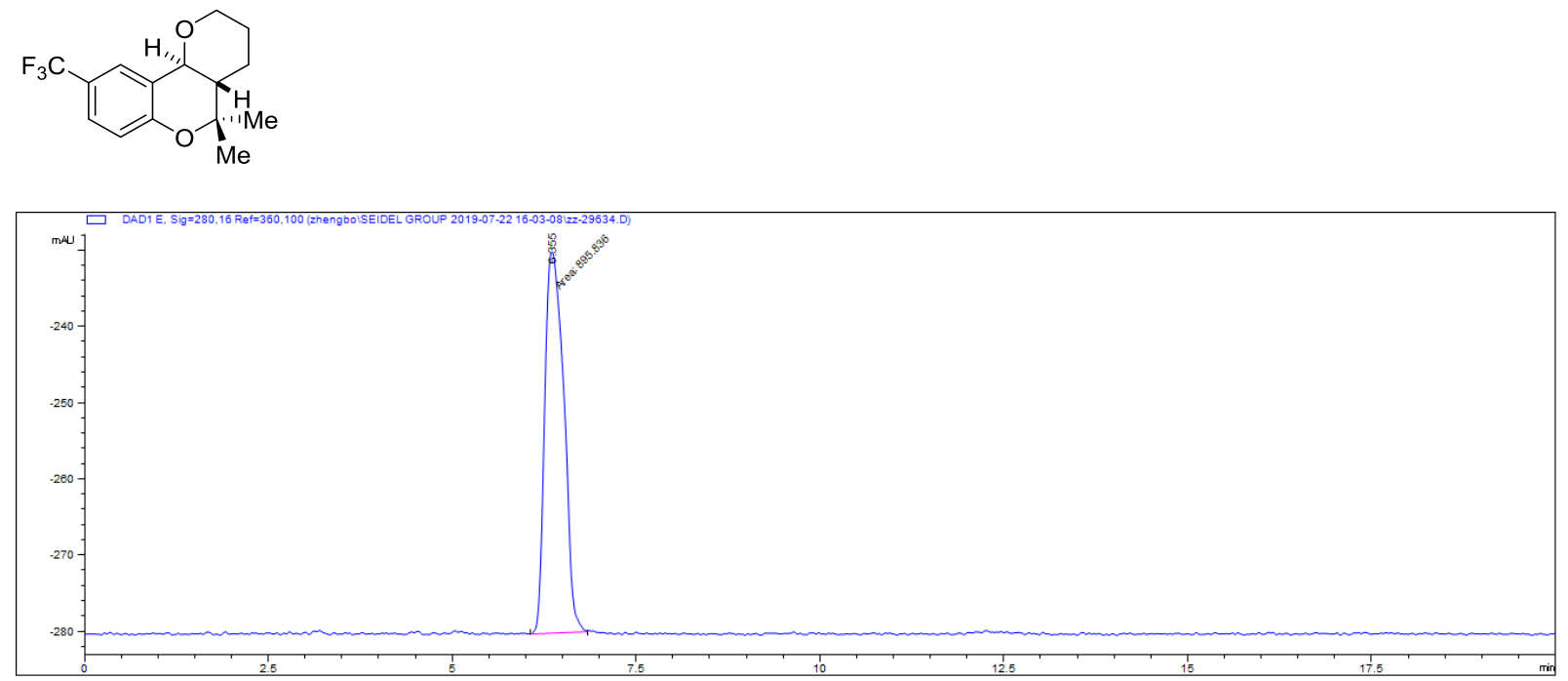

\begin{tabular}{|l|l|l|}
\hline Peak & Retention time (min) & Area \% \\
\hline 1 & 6.355 & 100 \\
\hline
\end{tabular}

\section{Racemic sample}

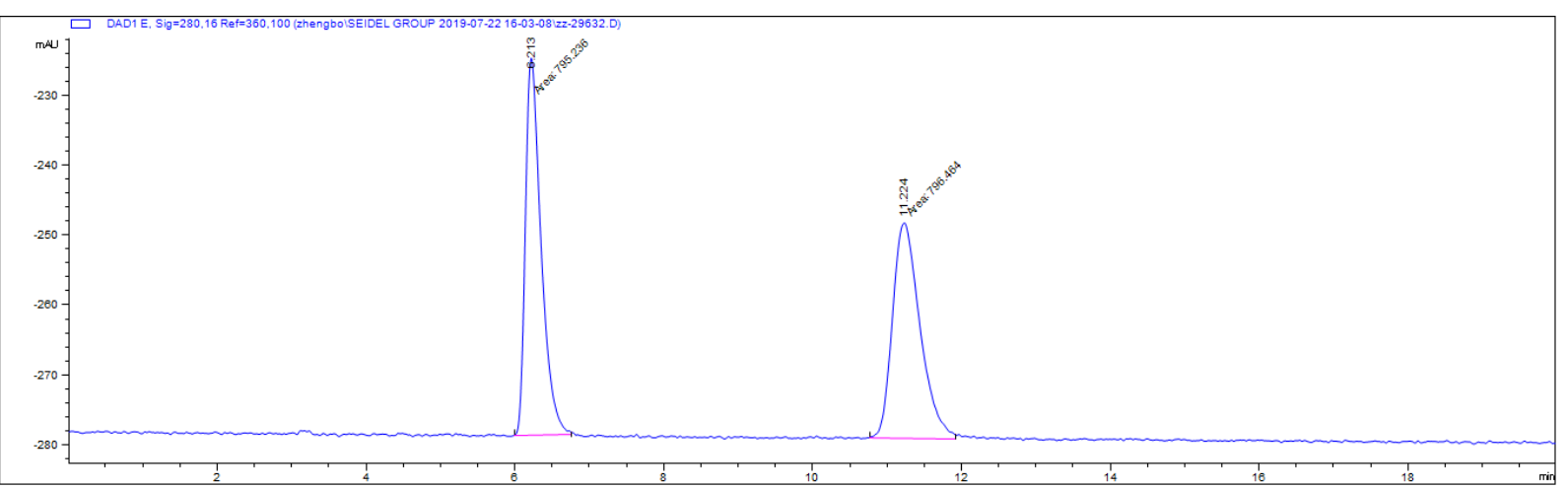

\begin{tabular}{|l|l|l|}
\hline Peak & Retention time (min) & Area \% \\
\hline 1 & 6.213 & 49.961 \\
\hline 2 & 11.224 & 50.039 \\
\hline
\end{tabular}




\section{SFC Profile of 3m:}

Conditions: Daicel Chiralcel AD-H, column temperature $=40{ }^{\circ} \mathrm{C}, \mathrm{CO}_{2} / \mathrm{MeOH}=90 / 10$, Flow rate $=2 \mathrm{~mL} / \mathrm{min}$, UV $=230 \mathrm{~nm}, \mathrm{t}_{\mathrm{R}}=5.0 \mathrm{~min}\left(\right.$ minor) and $\mathrm{t}_{\mathrm{R}}=5.3 \min$ (major).
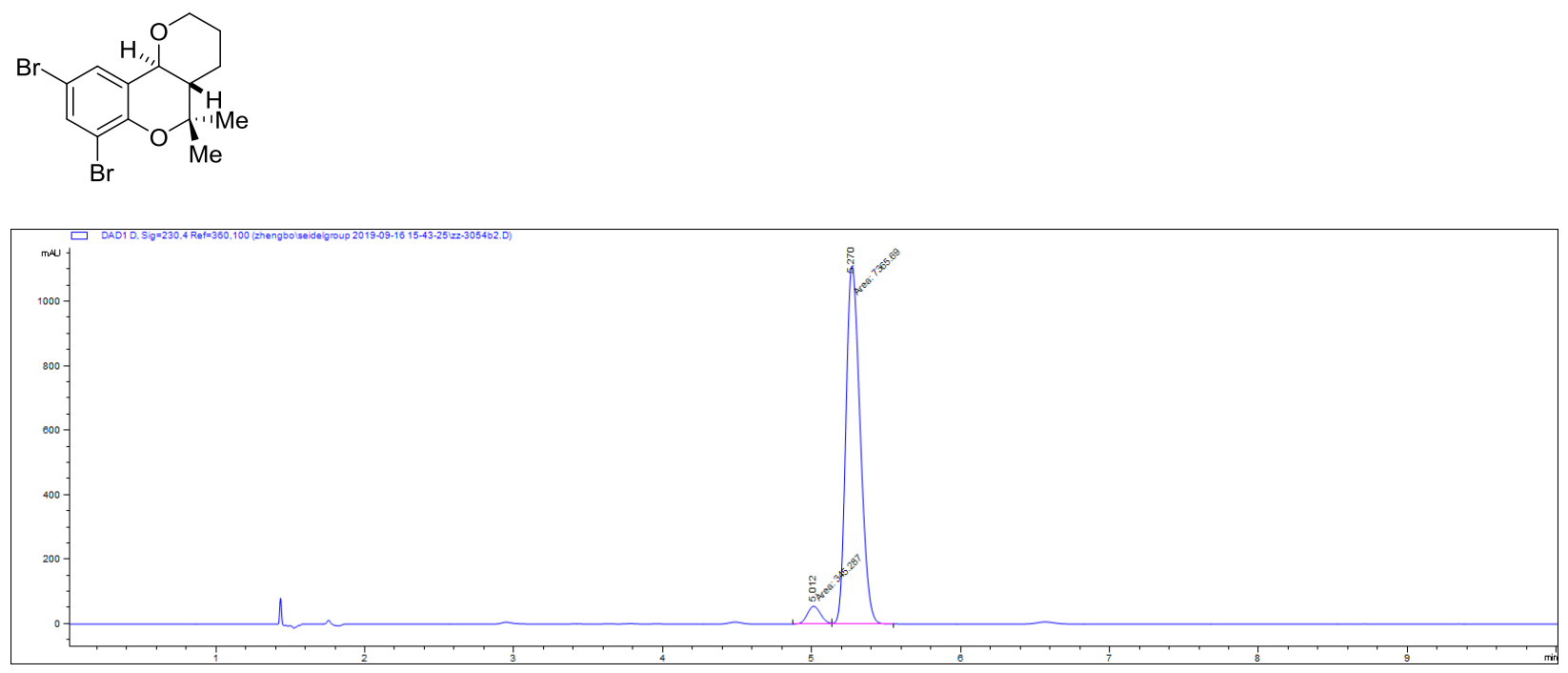

\begin{tabular}{|l|l|l|}
\hline Peak & Retention time (min) & Area \% \\
\hline 1 & 5.012 & 4.478 \\
\hline 2 & 5.270 & 95.522 \\
\hline
\end{tabular}

\section{Racemic sample}

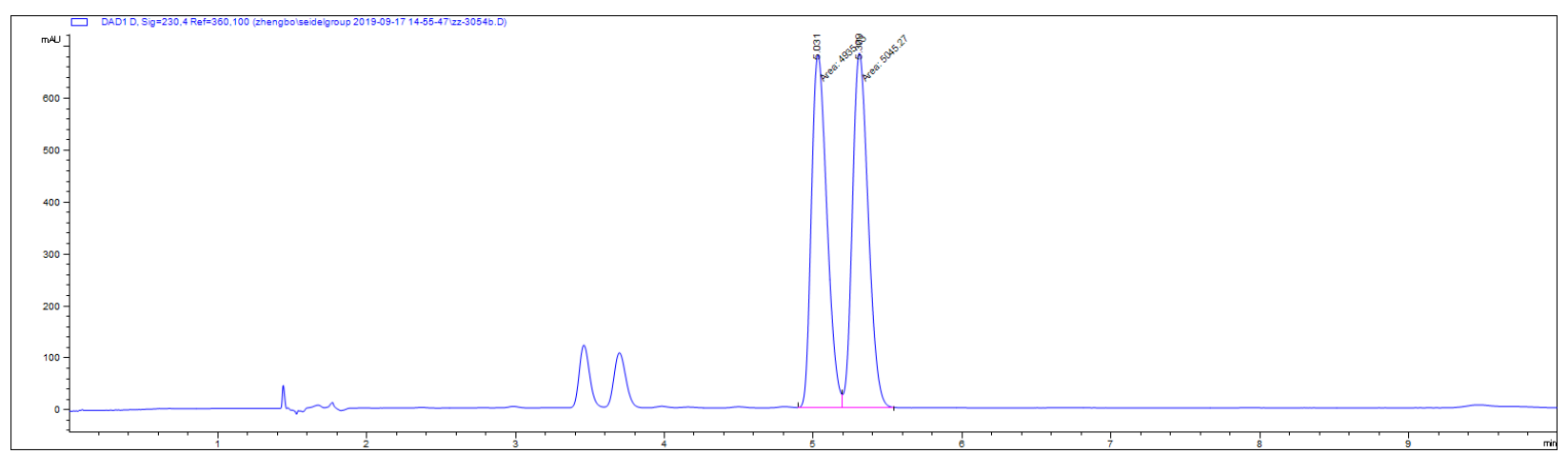

\begin{tabular}{|l|l|l|}
\hline Peak & Retention time (min) & Area \% \\
\hline 1 & 5.031 & 49.451 \\
\hline 2 & 5.309 & 50.549 \\
\hline
\end{tabular}




\section{SFC Profile of 3n:}

Conditions: Daicel Chiralcel AD-H, column temperature $=40{ }^{\circ} \mathrm{C}, \mathrm{CO}_{2} / \mathrm{MeOH}=95 / 5$, Flow rate $=2 \mathrm{~mL} / \mathrm{min}, \mathrm{UV}=$ $280 \mathrm{~nm}, \mathrm{t}_{\mathrm{R}}=7.2 \min$ (minor) and $\mathrm{t}_{\mathrm{R}}=10.4 \min$ (major).
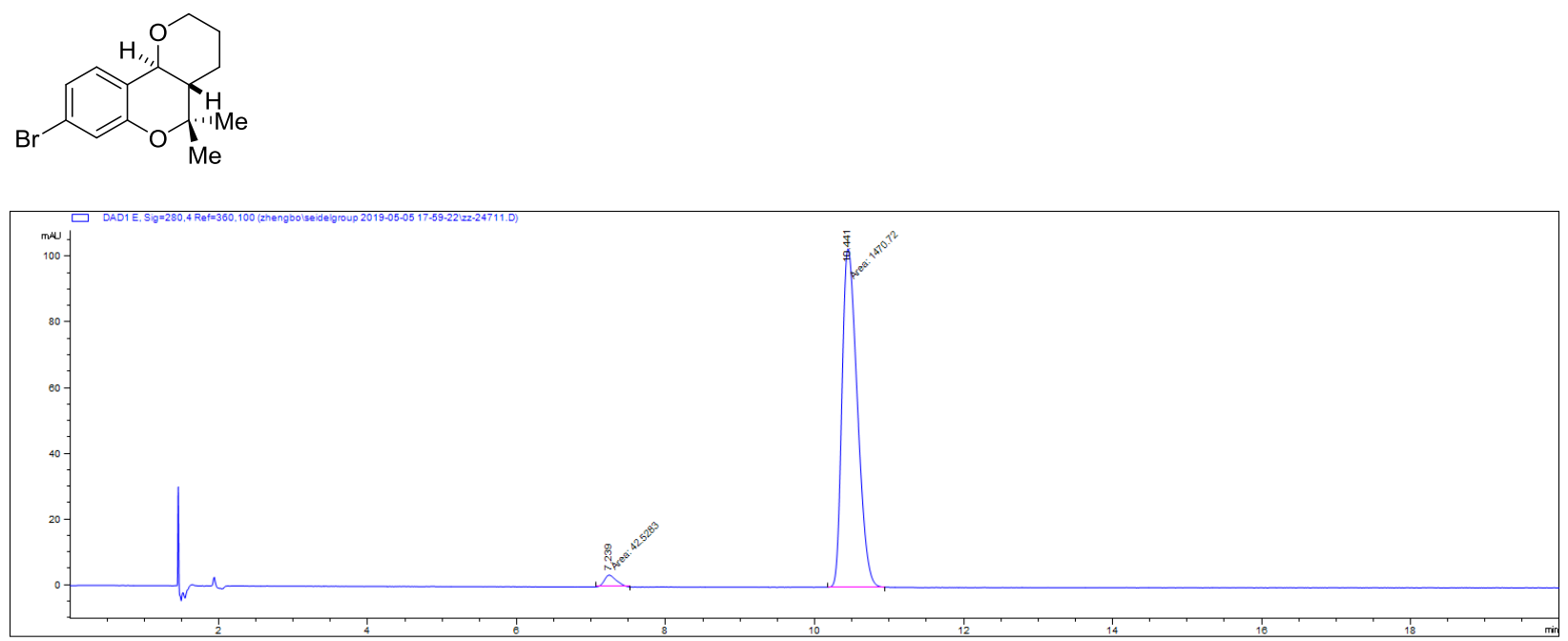

\begin{tabular}{|l|l|l|}
\hline Peak & Retention time (min) & Area \% \\
\hline 1 & 7.239 & 2.810 \\
\hline 2 & 10.441 & 97.190 \\
\hline
\end{tabular}

\section{Racemic sample}

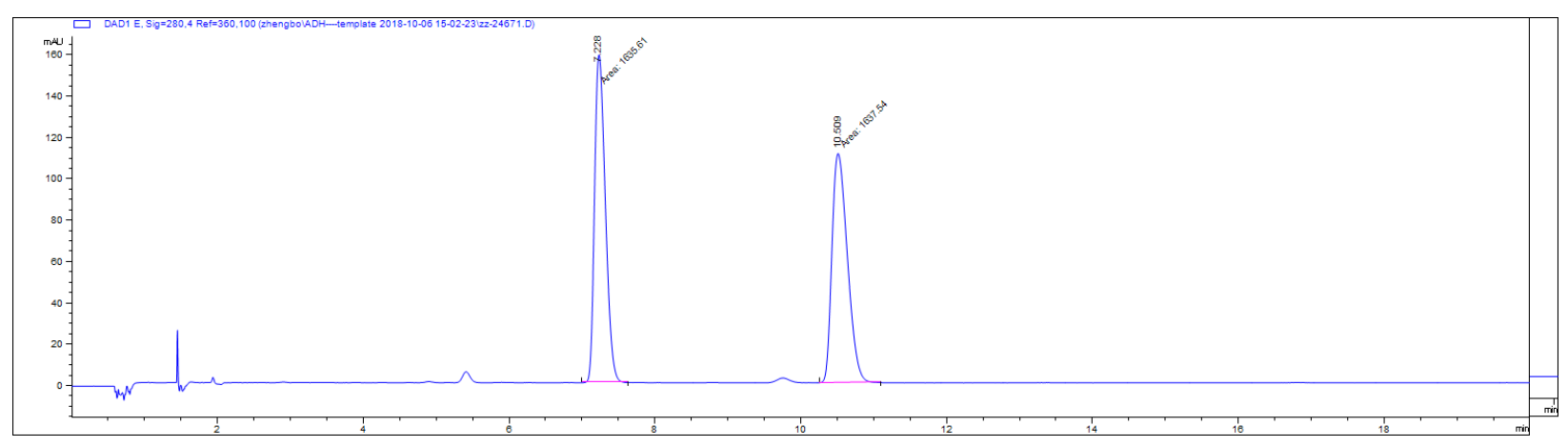

\begin{tabular}{|l|l|l|}
\hline Peak & Retention time (min) & Area \% \\
\hline 1 & 7.228 & 49.971 \\
\hline 2 & 10.509 & 50.029 \\
\hline
\end{tabular}




\section{SFC Profile of 3o:}

Conditions: Daicel Chiralcel OJ-H, column temperature $=40{ }^{\circ} \mathrm{C}, \mathrm{CO}_{2} / \mathrm{MeOH}=80 / 20$, Flow rate $=2 \mathrm{~mL} / \mathrm{min}, \mathrm{UV}=$ $230 \mathrm{~nm}, \mathrm{t}_{\mathrm{R}}=3.7 \mathrm{~min}$ (minor) and $\mathrm{t}_{\mathrm{R}}=4.6 \mathrm{~min}$ (major).
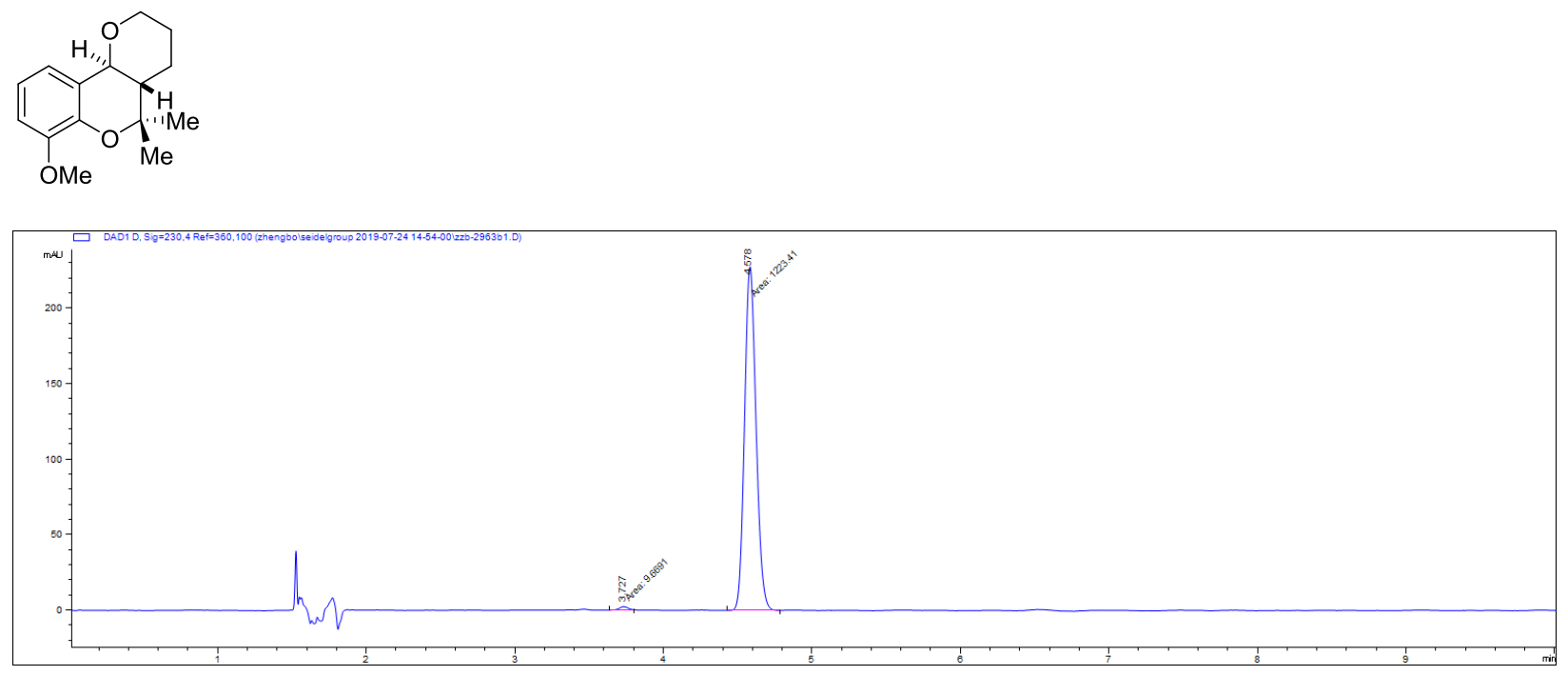

\begin{tabular}{|l|l|l|}
\hline Peak & Retention time (min) & Area \% \\
\hline 1 & 3.727 & 0.794 \\
\hline 2 & 4.578 & 99.216 \\
\hline
\end{tabular}

Racemic sample

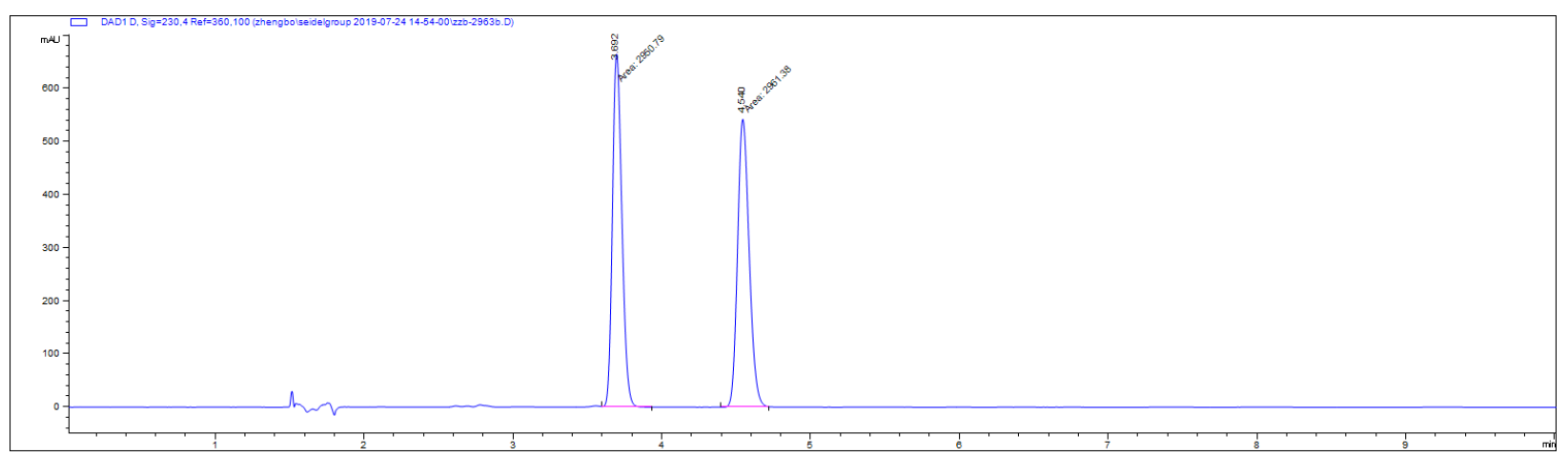

\begin{tabular}{|l|l|l|}
\hline Peak & Retention time (min) & Area \% \\
\hline 1 & 3.692 & 49.910 \\
\hline 2 & 4.540 & 50.090 \\
\hline
\end{tabular}




\section{SFC Profile of 3p:}

Conditions: Daicel Chiralcel OJ-H, column temperature $=40{ }^{\circ} \mathrm{C}, \mathrm{CO}_{2} / \mathrm{MeOH}=80 / 20$, Flow rate $=2 \mathrm{~mL} / \mathrm{min}, \mathrm{UV}=$ $280 \mathrm{~nm}, \mathrm{t}_{\mathrm{R}}=4.4 \min$ (major) and $\mathrm{t}_{\mathrm{R}}=7.2 \mathrm{~min}$ (minor).
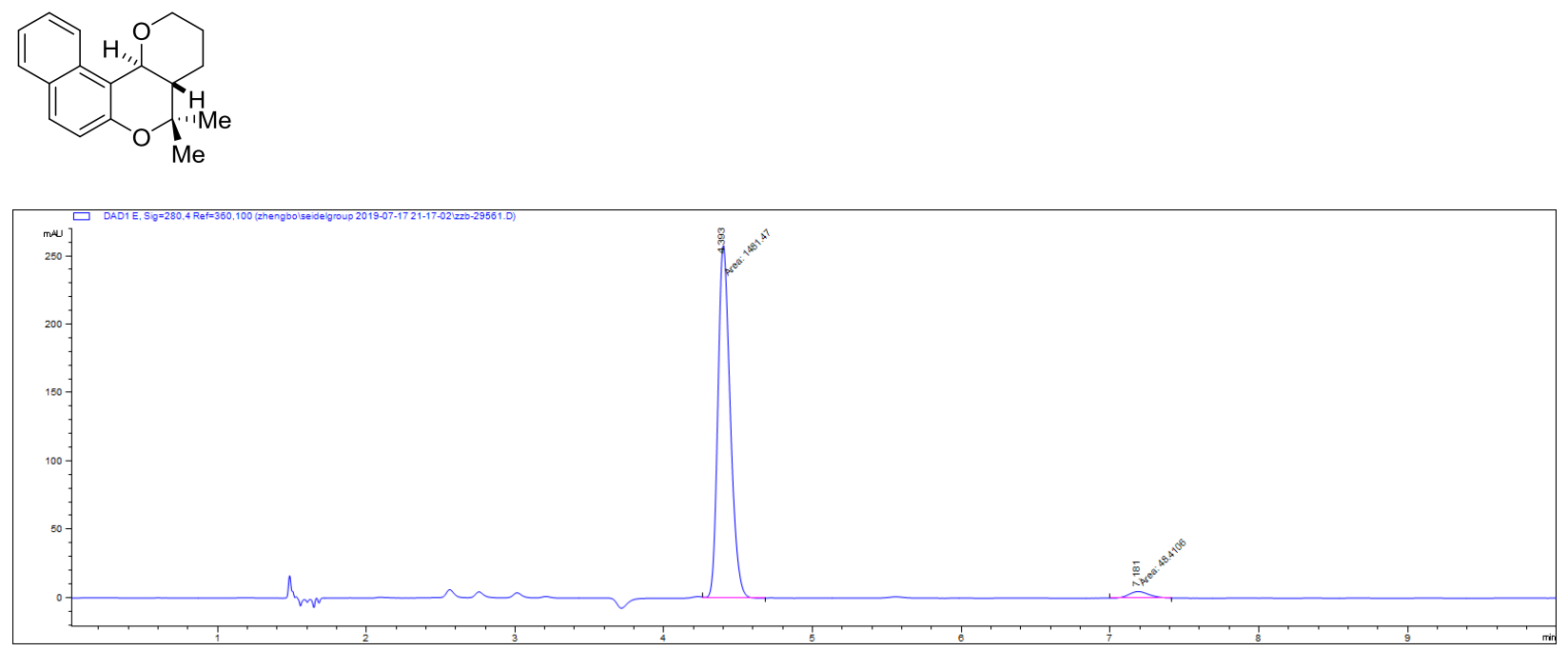

\begin{tabular}{|l|l|l|}
\hline Peak & Retention time (min) & Area \% \\
\hline 1 & 4.393 & 96.836 \\
\hline 2 & 7.181 & 3.164 \\
\hline
\end{tabular}

Racemic sample

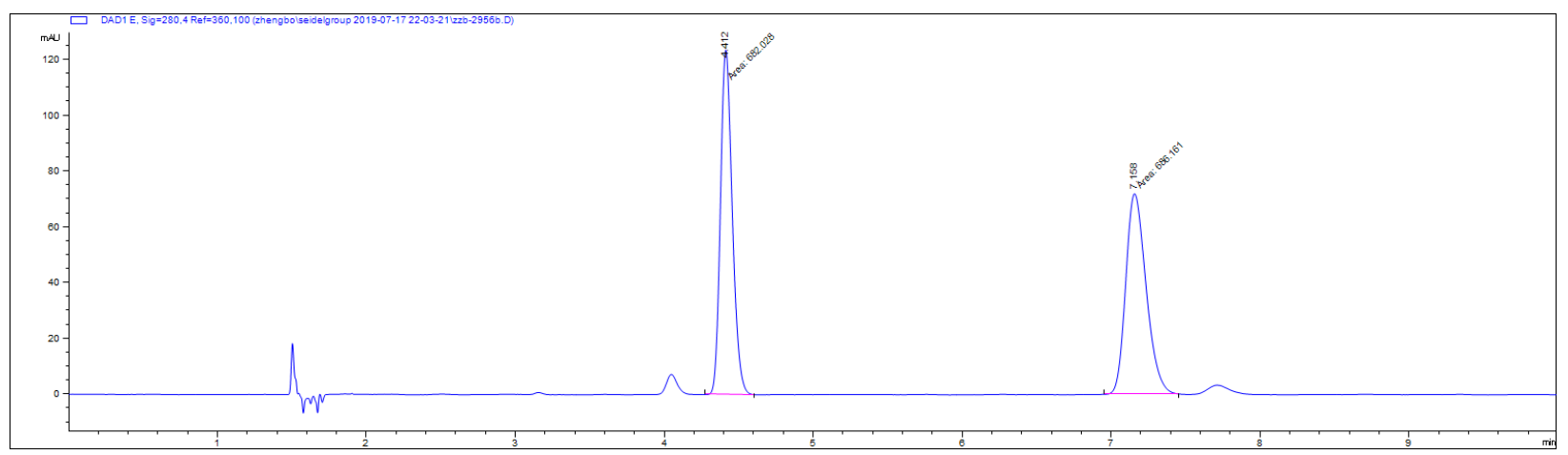

\begin{tabular}{|l|l|l|}
\hline Peak & Retention time (min) & Area \% \\
\hline 1 & 4.412 & 49.849 \\
\hline 2 & 7.158 & 50.151 \\
\hline
\end{tabular}




\section{SFC Profile of 7:}

Conditions: Daicel Chiralcel OJ-H, column temperature $=40{ }^{\circ} \mathrm{C}, \mathrm{CO}_{2} / \mathrm{MeOH}=90 / 10$, Flow rate $=2 \mathrm{~mL} / \mathrm{min}, \mathrm{UV}=$ $230 \mathrm{~nm}, \mathrm{t}_{\mathrm{R}}=3.3 \min$ (major) and $\mathrm{t}_{\mathrm{R}}=3.9 \min$ (minor).
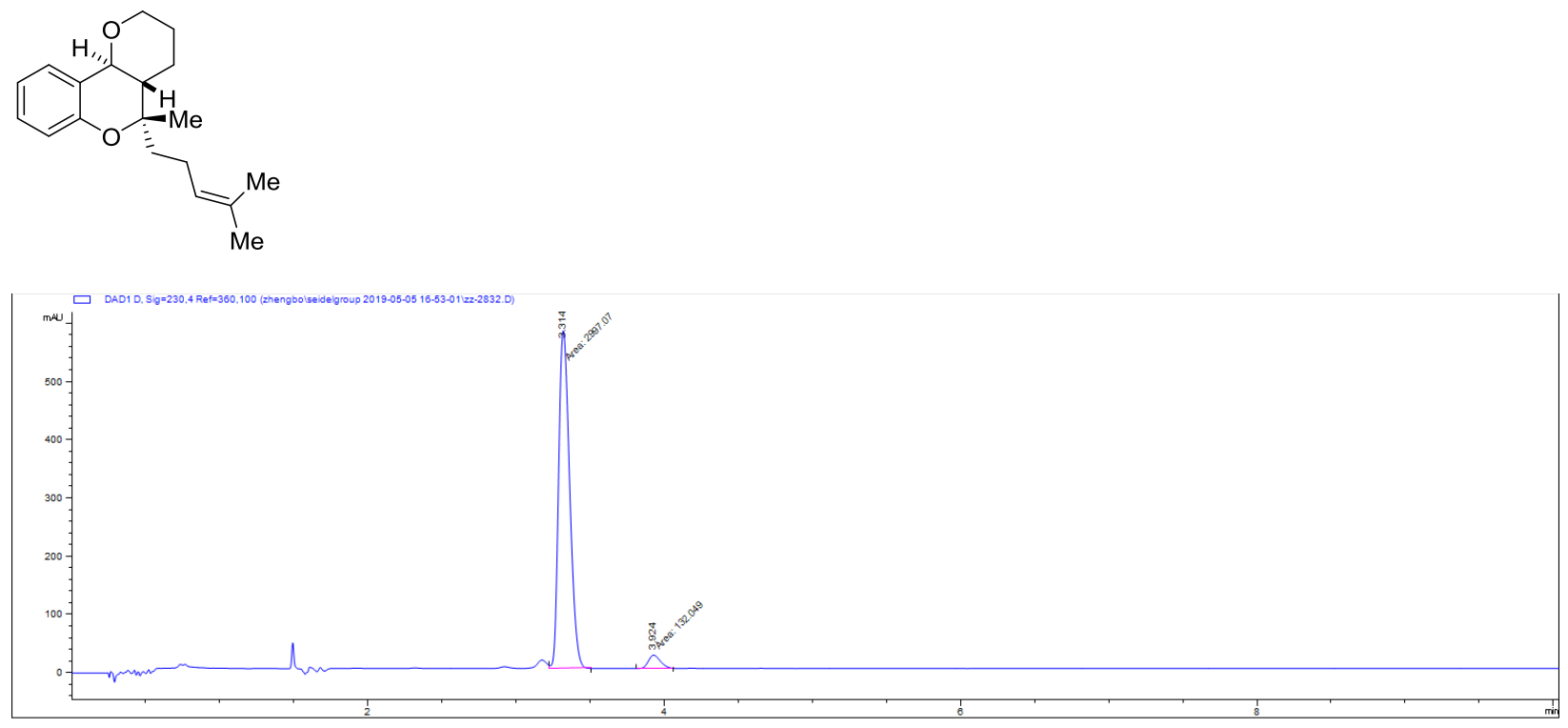

\begin{tabular}{|l|l|l|}
\hline Peak & Retention time (min) & Area \% \\
\hline 1 & 3.314 & 95.780 \\
\hline 2 & 3.924 & 4.220 \\
\hline
\end{tabular}

\section{Racemic sample}

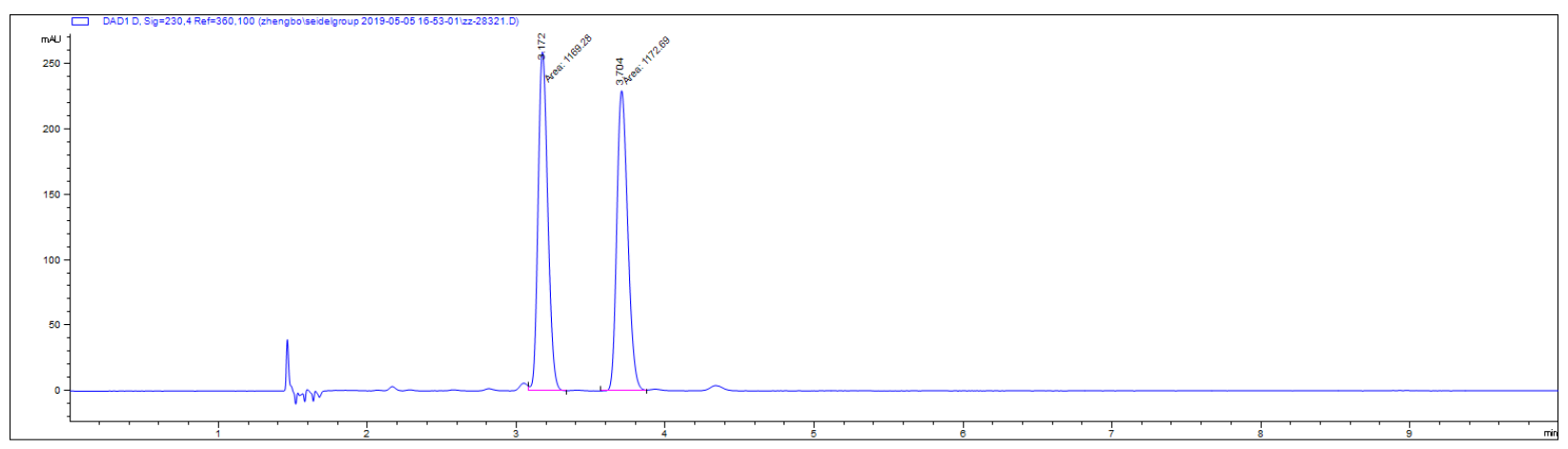

\begin{tabular}{|l|l|l|}
\hline Peak & Retention time (min) & Area \% \\
\hline 1 & 3.172 & 49.927 \\
\hline 2 & 3.704 & 50.073 \\
\hline
\end{tabular}




\section{SFC Profile of 9:}

Conditions: Daicel Chiralcel OJ-H, column temperature $=40{ }^{\circ} \mathrm{C}, \mathrm{CO}_{2} / \mathrm{MeOH}=90 / 10$, Flow rate $=2 \mathrm{~mL} / \mathrm{min}, \mathrm{UV}=$ $280 \mathrm{~nm}, \mathrm{t}_{\mathrm{R}}=3.1 \mathrm{~min}$ (major) and $\mathrm{t}_{\mathrm{R}}=3.8 \mathrm{~min}$ (minor).
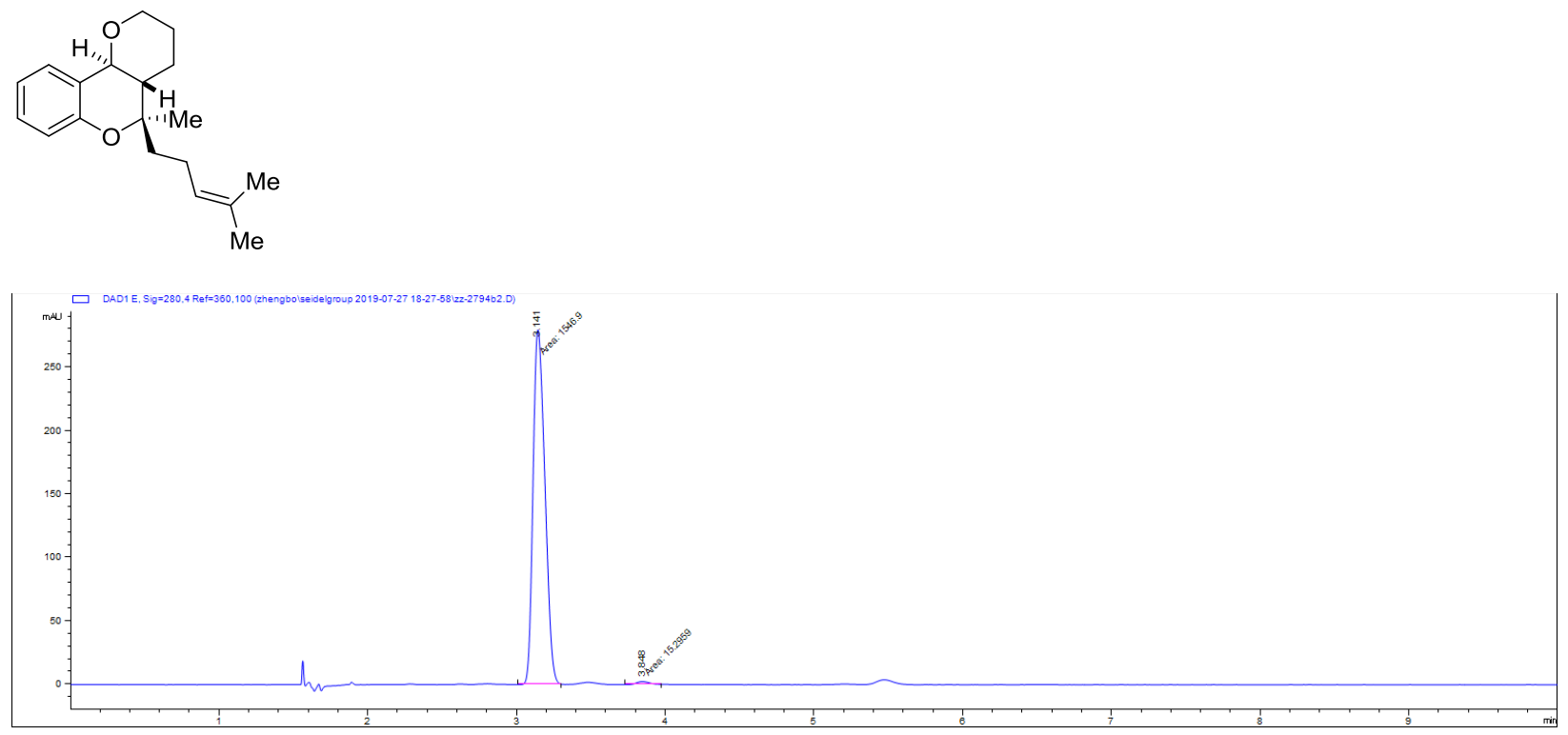

\begin{tabular}{|l|l|l|}
\hline Peak & Retention time (min) & Area \% \\
\hline 1 & 3.141 & 99.021 \\
\hline 2 & 3.848 & 0.979 \\
\hline
\end{tabular}

Racemic sample

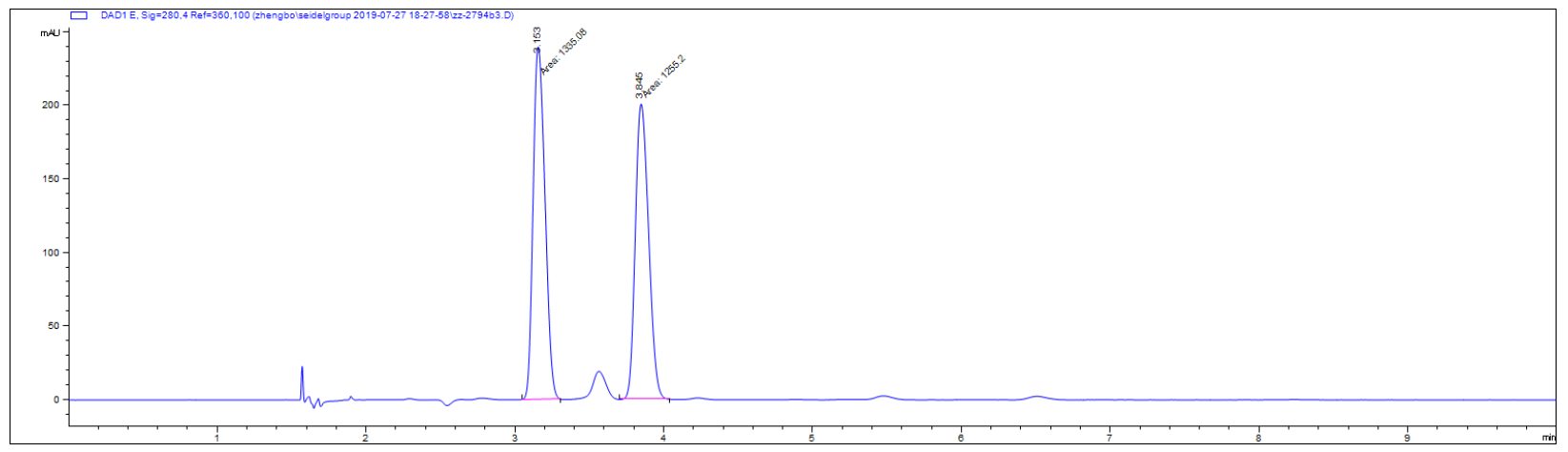

\begin{tabular}{|l|l|l|}
\hline Peak & Retention time (min) & Area \% \\
\hline 1 & 3.153 & 51.542 \\
\hline 2 & 3.845 & 48.458 \\
\hline
\end{tabular}




\section{SFC Profile of 11:}

Conditions: Daicel Chiralcel AD-H, column temperature $=40{ }^{\circ} \mathrm{C}, \mathrm{CO}_{2} / \mathrm{MeOH}=97 / 3$, Flow rate $=2 \mathrm{~mL} / \mathrm{min}, \mathrm{UV}=$ $280 \mathrm{~nm}, \mathrm{t}_{\mathrm{R}}=3.2 \mathrm{~min}$ (minor) and $\mathrm{t}_{\mathrm{R}}=3.5 \mathrm{~min}$ (major).
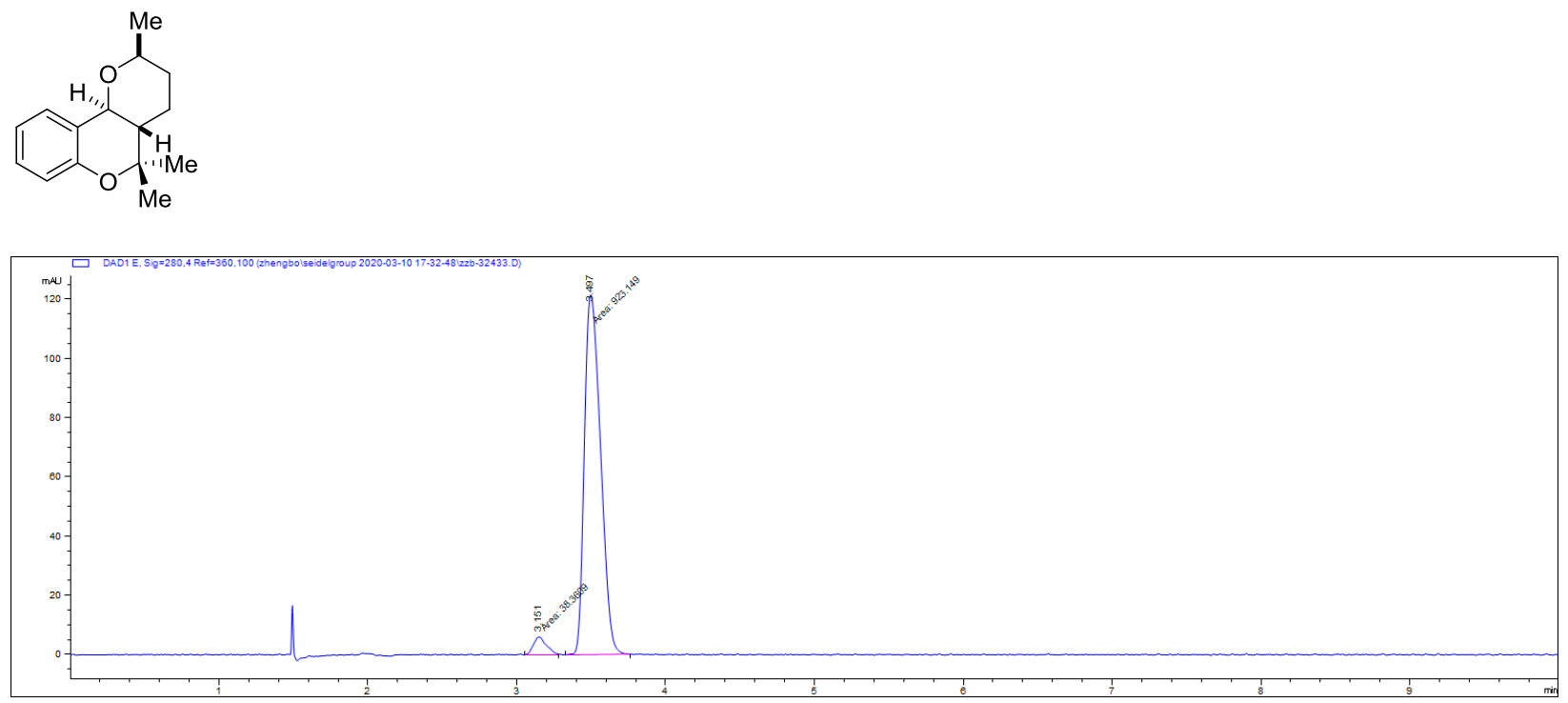

\begin{tabular}{|l|l|l|}
\hline Peak & Retention time (min) & Area \% \\
\hline 1 & 3.151 & 3.990 \\
\hline 2 & 3.497 & 96.010 \\
\hline
\end{tabular}

Racemic sample

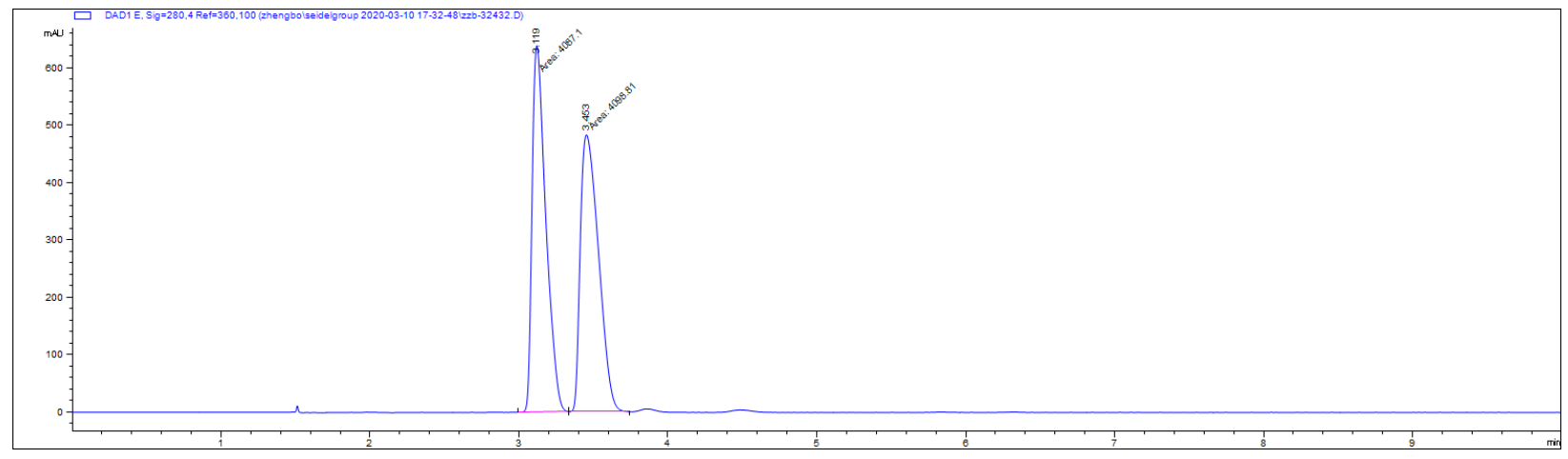

\begin{tabular}{|l|l|l|}
\hline Peak & Retention time (min) & Area \% \\
\hline 1 & 3.119 & 49.929 \\
\hline 2 & 3.453 & 50.071 \\
\hline
\end{tabular}




\section{SFC Profile of 13:}

Conditions: Daicel Chiralcel OJ-H, column temperature $=40{ }^{\circ} \mathrm{C}, \mathrm{CO}_{2} / \mathrm{MeOH}=90 / 10$, Flow rate $=2 \mathrm{~mL} / \mathrm{min}, \mathrm{UV}=$ $280 \mathrm{~nm}, \mathrm{t}_{\mathrm{R}}=7.0 \mathrm{~min}$ (minor) and $\mathrm{t}_{\mathrm{R}}=8.0 \mathrm{~min}$ (major).
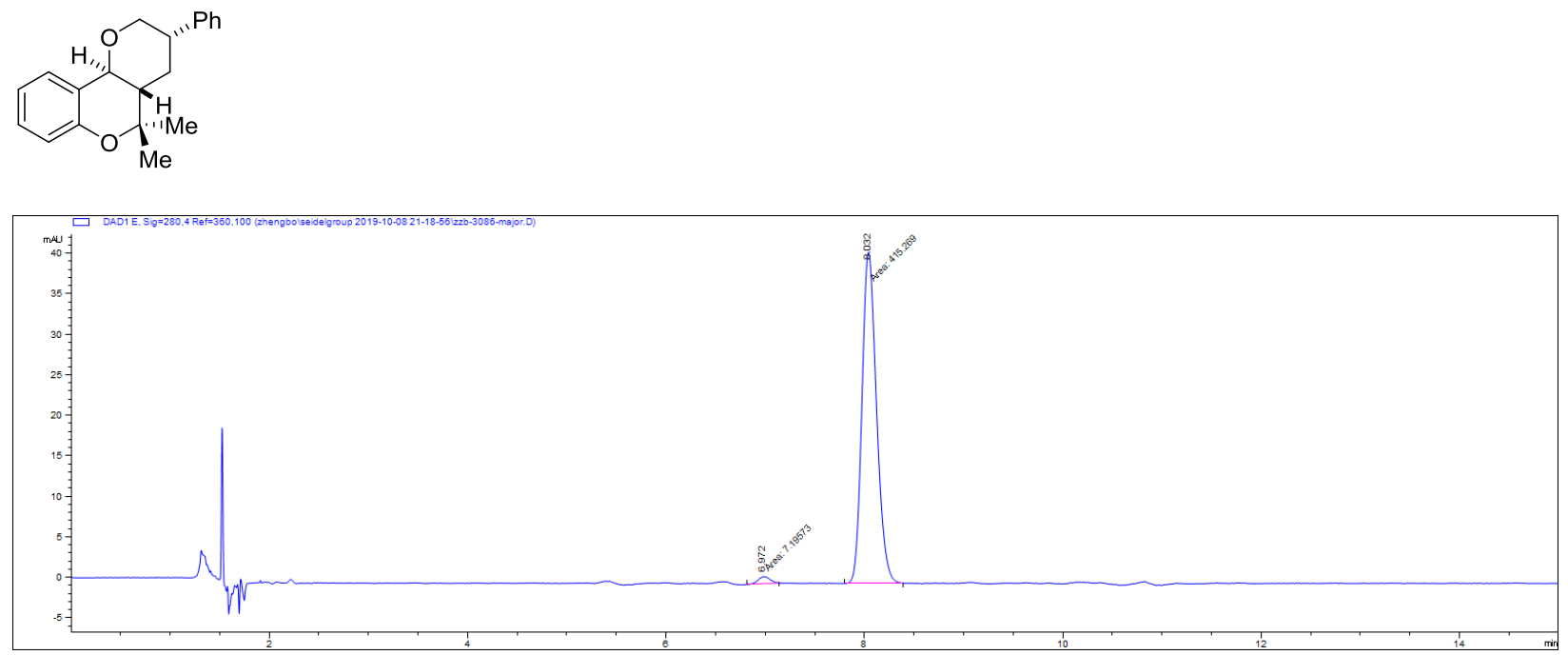

\begin{tabular}{|l|l|l|}
\hline Peak & Retention time (min) & Area \% \\
\hline 1 & 6.972 & 1.703 \\
\hline 2 & 8.032 & 98.297 \\
\hline
\end{tabular}

\section{Racemic sample}

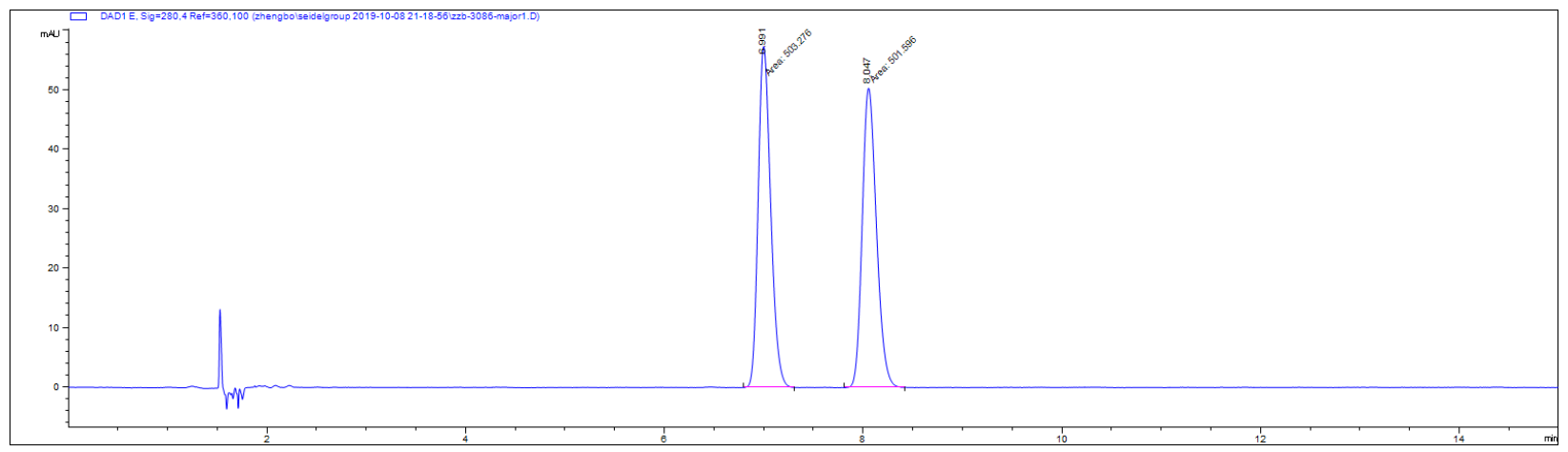

\begin{tabular}{|l|l|l|}
\hline Peak & Retention time (min) & Area \% \\
\hline 1 & 6.991 & 50.084 \\
\hline 2 & 8.047 & 49.916 \\
\hline
\end{tabular}




\section{SFC Profile of 13'}

Conditions: Daicel Chiralcel AD-H, column temperature $=40{ }^{\circ} \mathrm{C}, \mathrm{CO}_{2} / \mathrm{MeOH}=90 / 10$, Flow rate $=2 \mathrm{~mL} / \mathrm{min}$, UV $=210 \mathrm{~nm}, \mathrm{t}_{\mathrm{R}}=3.4 \min$ (major).
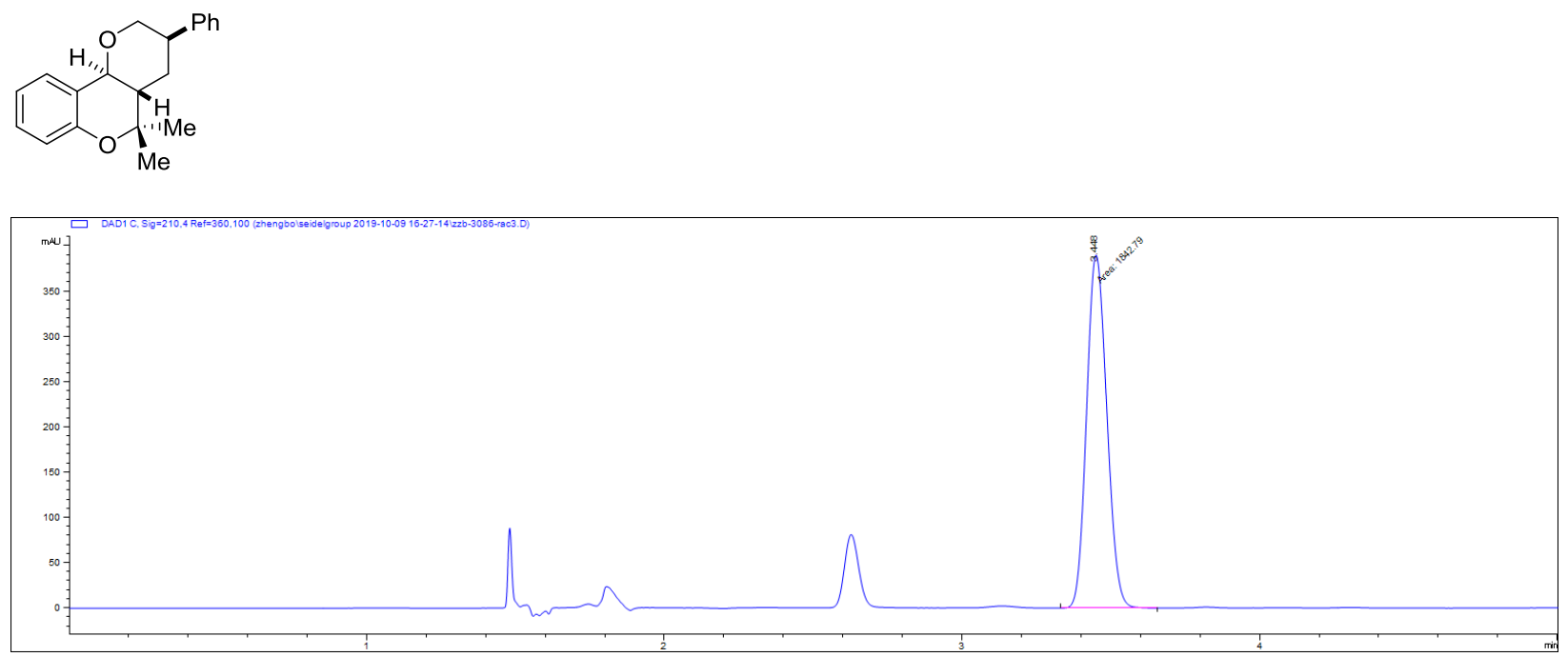

\begin{tabular}{|l|l|l|}
\hline Peak & Retention time (min) & Area \% \\
\hline 1 & 3.448 & 100 \\
\hline
\end{tabular}

\section{Racemic sample}

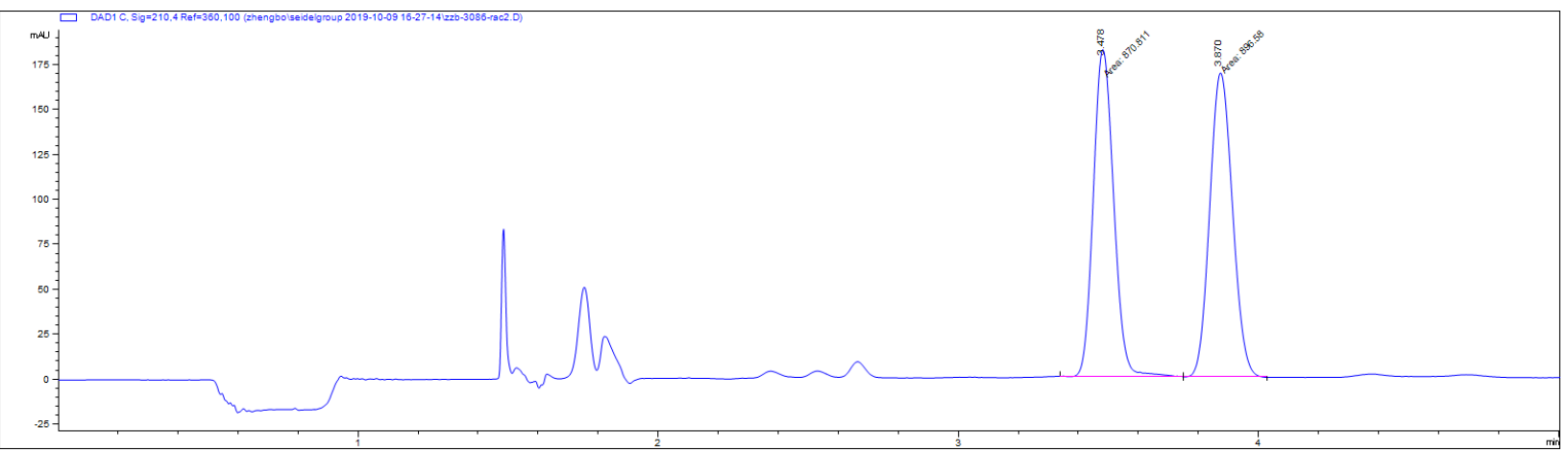

\begin{tabular}{|l|l|l|}
\hline Peak & Retention time (min) & Area \% \\
\hline 1 & 3.478 & 50.035 \\
\hline 2 & 3.870 & 49.965 \\
\hline
\end{tabular}




\section{SFC Profile of S2}

Conditions: Daicel Chiralcel AD-H, column temperature $=40{ }^{\circ} \mathrm{C}, \mathrm{CO}_{2} / \mathrm{MeOH}=90 / 10$, Flow rate $=2 \mathrm{~mL} / \mathrm{min}$, UV $=280 \mathrm{~nm}, \mathrm{t}_{\mathrm{R}}=6.3 \min \left(\right.$ minor) and $\mathrm{t}_{\mathrm{R}}=7.5 \min$ (major).
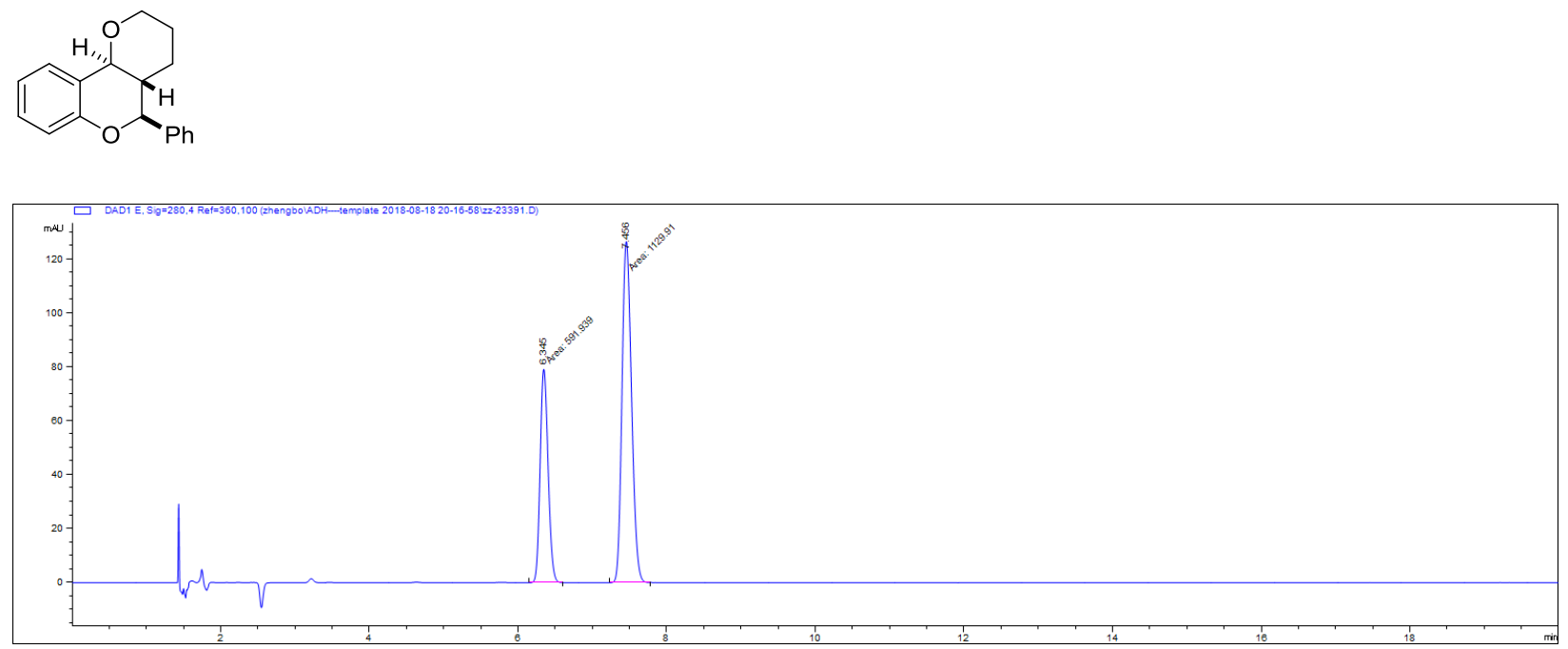

\begin{tabular}{|l|l|l|}
\hline \hline Peak & Retention time (min) & Area $\%$ \\
\hline 1 & 6.345 & 34.378 \\
\hline 2 & 7.456 & 65.622 \\
\hline
\end{tabular}

Racemic sample

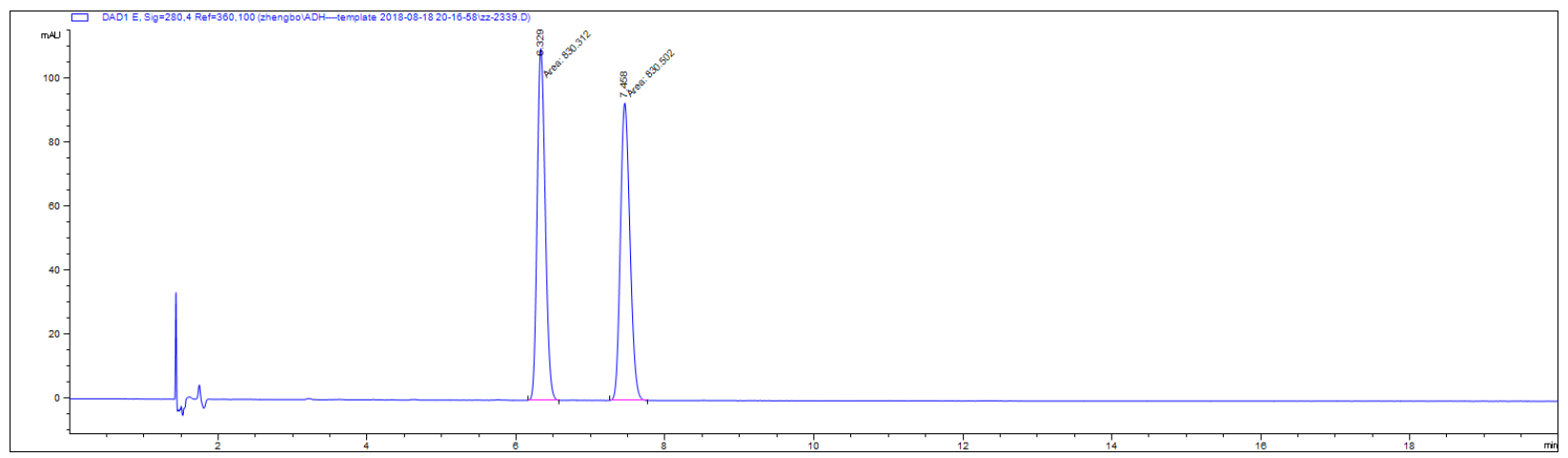

\begin{tabular}{|l|l|l|}
\hline Peak & Retention time (min) & Area \% \\
\hline 1 & 6.329 & 49.994 \\
\hline 2 & 7.458 & 50.006 \\
\hline
\end{tabular}




\section{SFC Profile of S4}

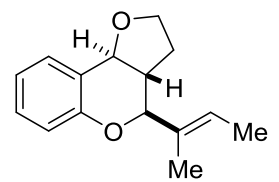

For the major diastereomer

Conditions: Daicel Chiralcel AD-H, column temperature $=40{ }^{\circ} \mathrm{C}, \mathrm{CO}_{2} / \mathrm{MeOH}=90 / 10$, Flow rate $=2 \mathrm{~mL} / \mathrm{min}$, UV $=280 \mathrm{~nm}, \mathrm{t}_{\mathrm{R}}=3.9 \mathrm{~min}$ (major) and $\mathrm{t}_{\mathrm{R}}=5.0 \mathrm{~min}$ (minor).

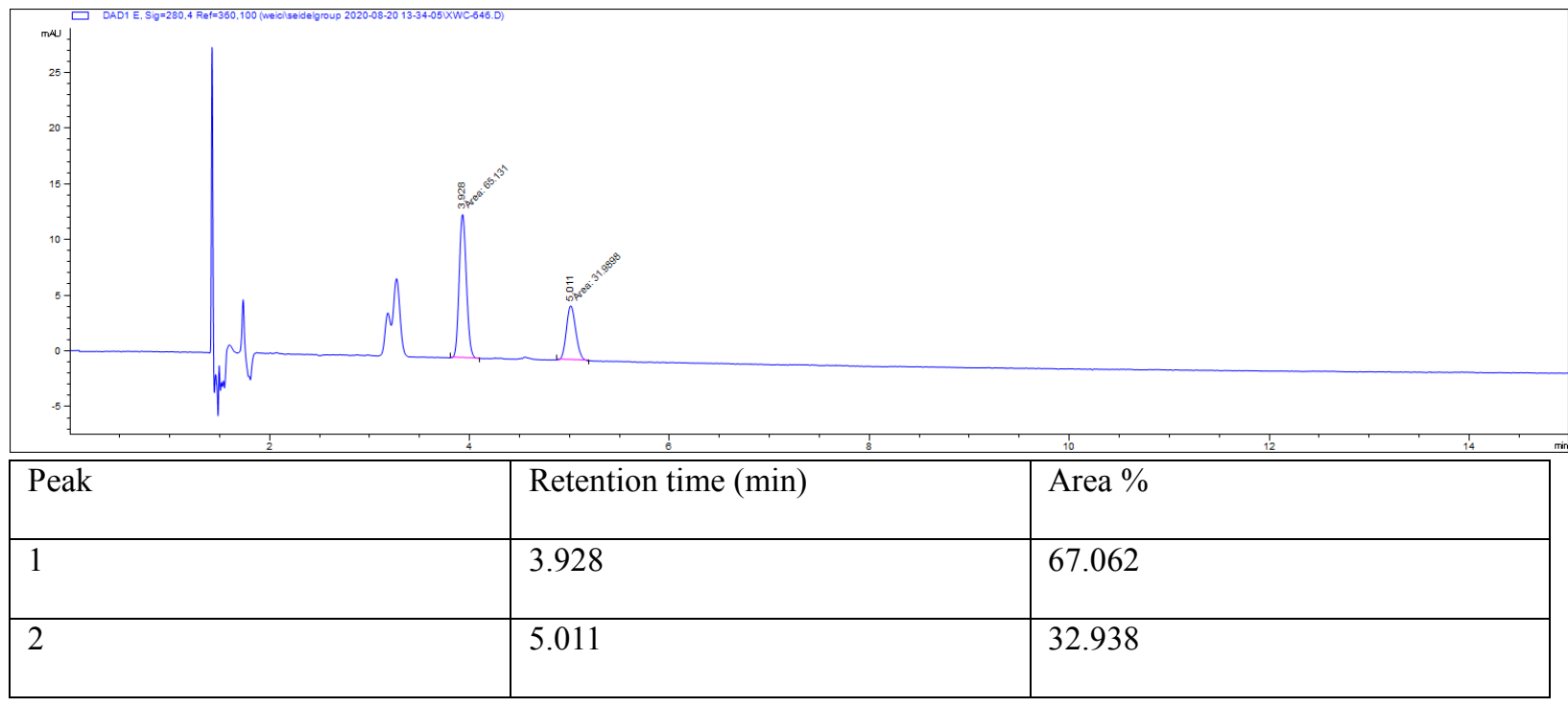

Racemic sample

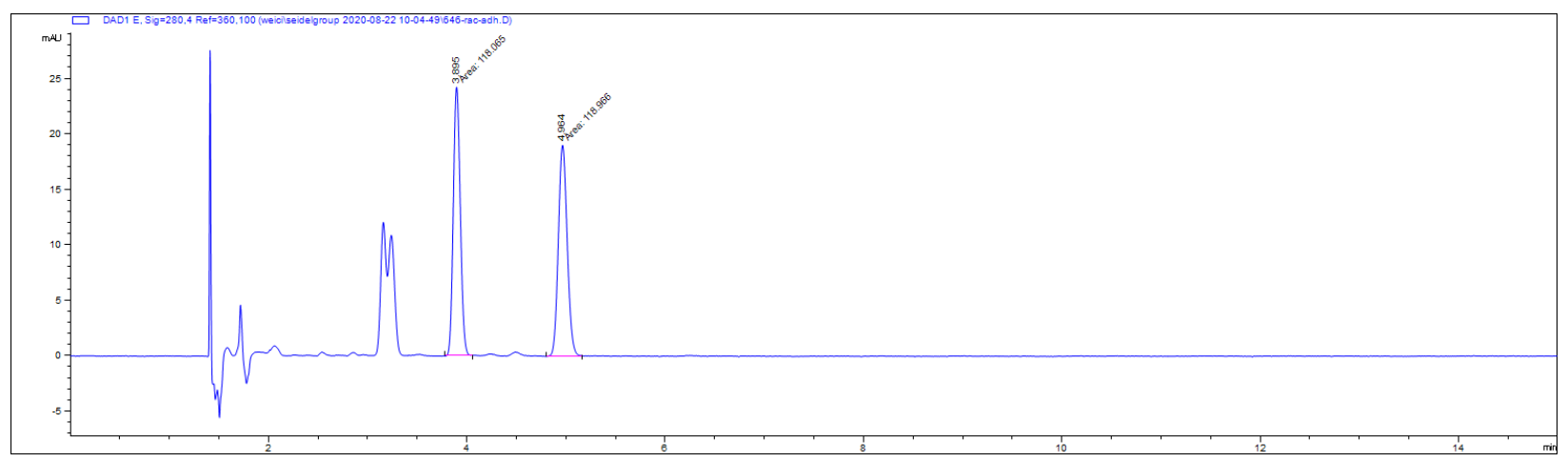

\begin{tabular}{|l|l|l|}
\hline Peak & Retention time (min) & Area \% \\
\hline 1 & 3.895 & 49.810 \\
\hline 2 & 4.964 & 50.190 \\
\hline
\end{tabular}


For the minor diastereomer

Conditions: Daicel Chiralcel OJ-H, column temperature $=40{ }^{\circ} \mathrm{C}, \mathrm{CO}_{2} / \mathrm{MeOH}=90 / 10$, Flow rate $=1 \mathrm{~mL} / \mathrm{min}$, UV $=$ $280 \mathrm{~nm}, \mathrm{t}_{\mathrm{R}}=7.6 \mathrm{~min}$ (major) and $\mathrm{t}_{\mathrm{R}}=7.9 \mathrm{~min}$ (minor).

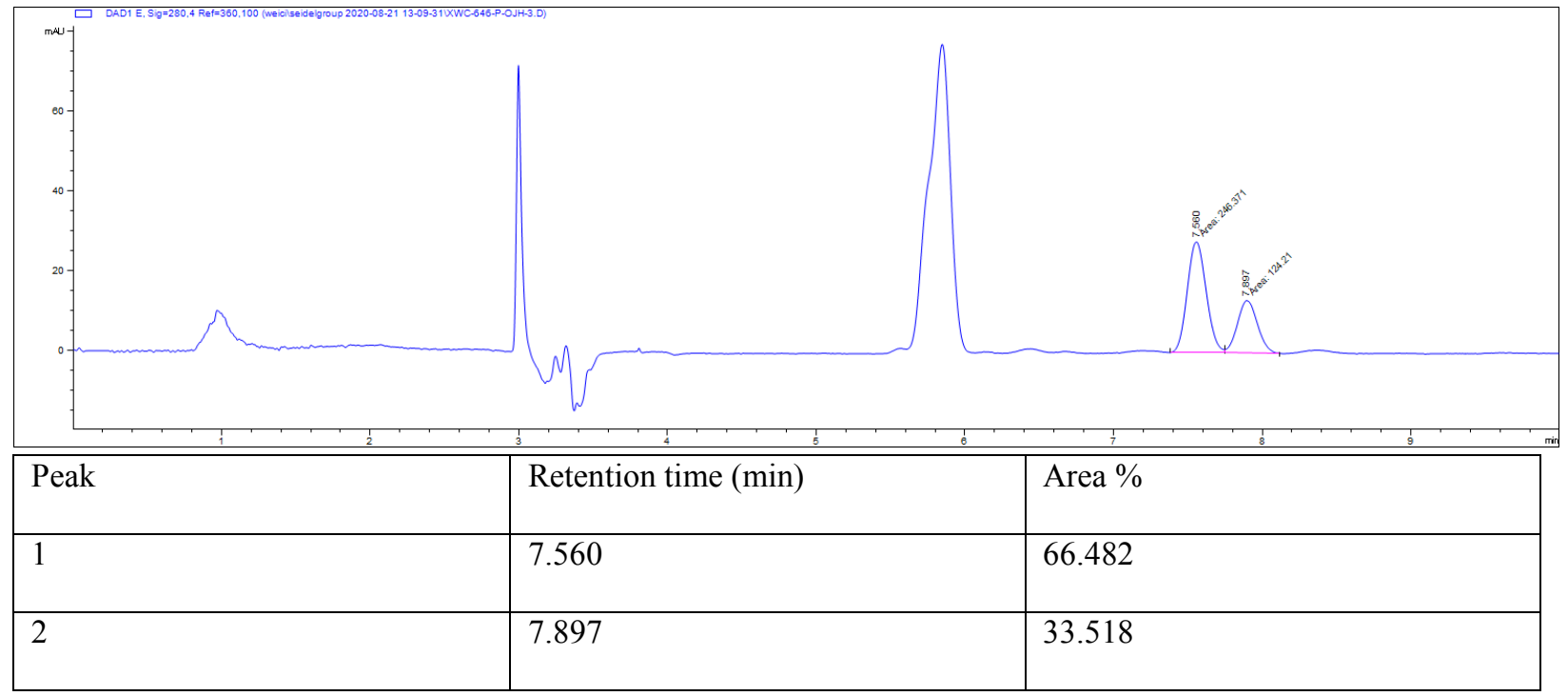

Racemic sample

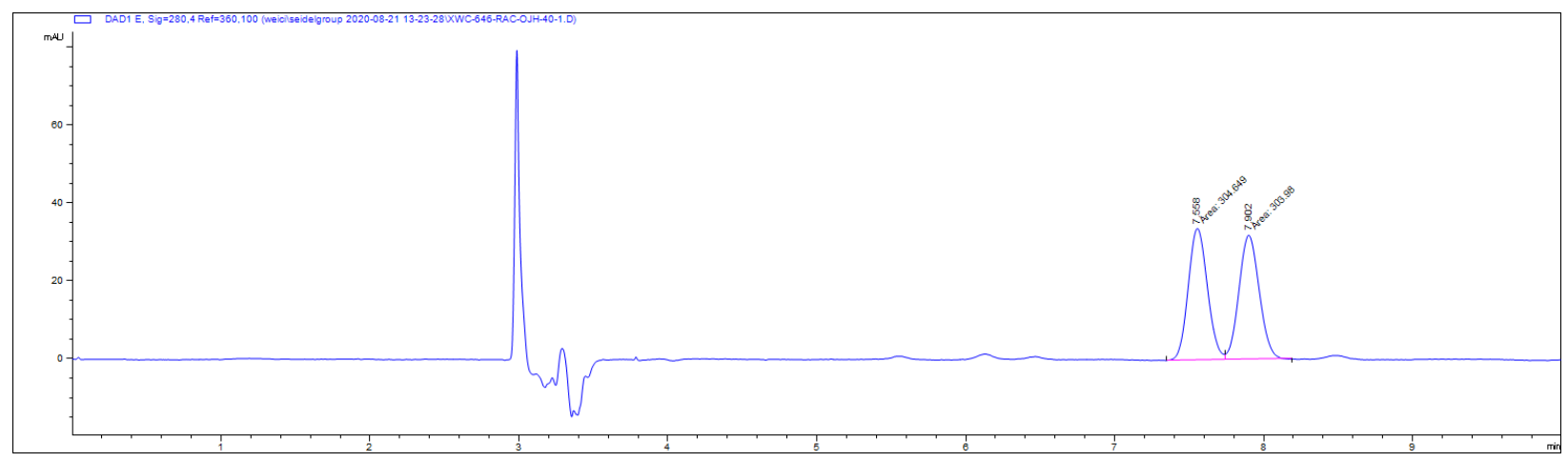

\begin{tabular}{|l|l|l|}
\hline Peak & Retention time (min) & Area \% \\
\hline 1 & 7.558 & 50.055 \\
\hline 2 & 7.902 & 49.945 \\
\hline
\end{tabular}




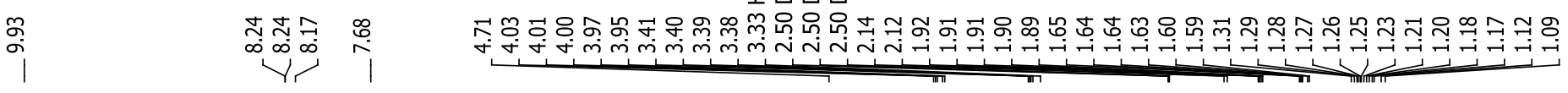

${ }^{1} \mathrm{H}-\mathrm{NMR}\left(600 \mathrm{MHz},\left(\mathrm{CD}_{3}\right)_{2} \mathrm{SO}\right)$ of $\mathbf{S} 5 \mathbf{b}$

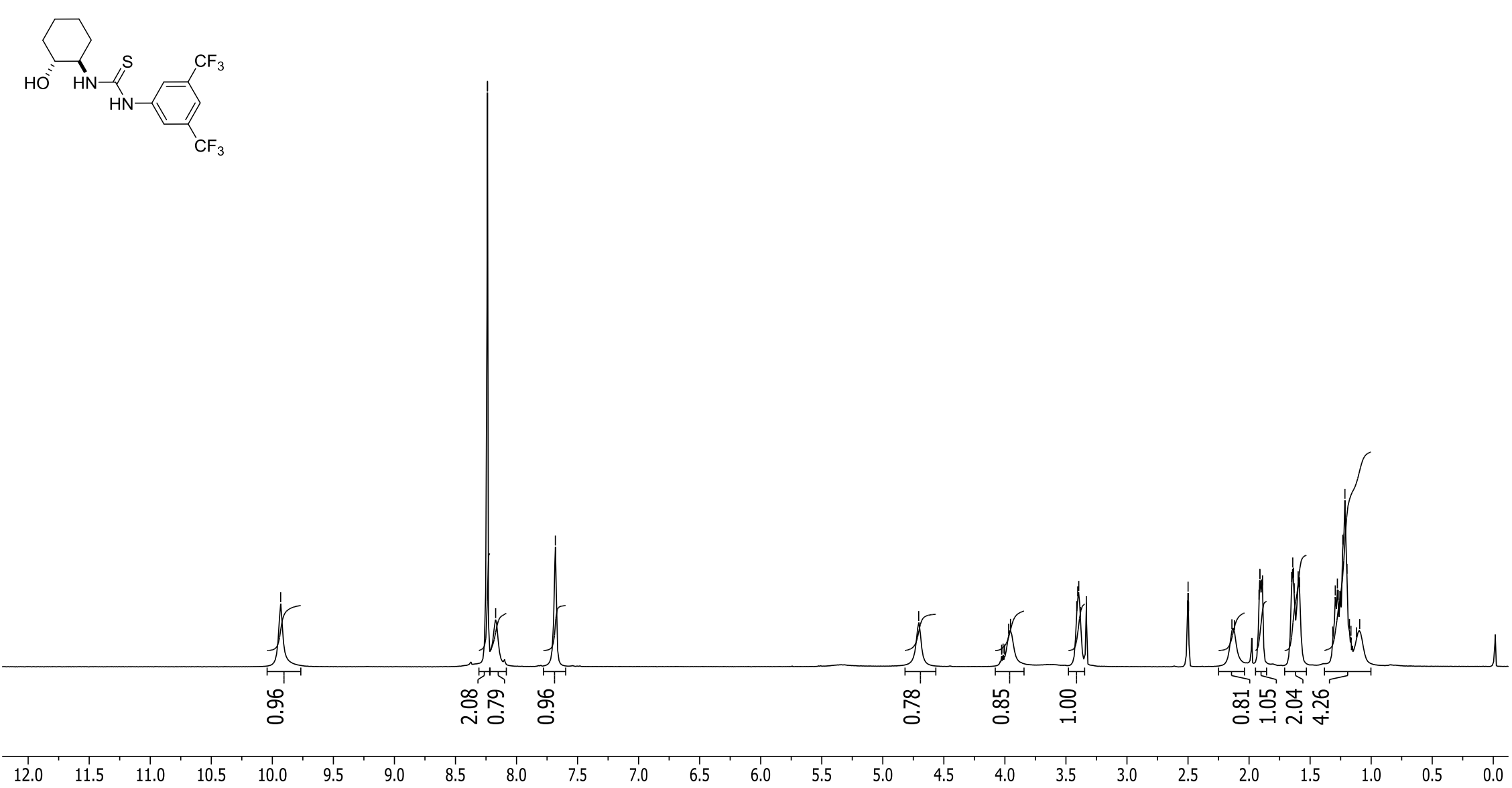




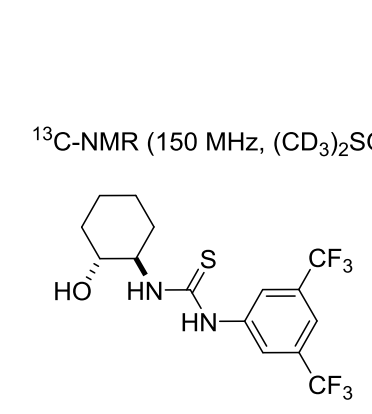

क

i

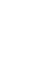


${ }^{19} \mathrm{~F}-\mathrm{NMR}\left(565 \mathrm{MHz},\left(\mathrm{CD}_{3}\right)_{2} \mathrm{SO}\right)$ of $\mathbf{S} 5 \mathbf{b}$

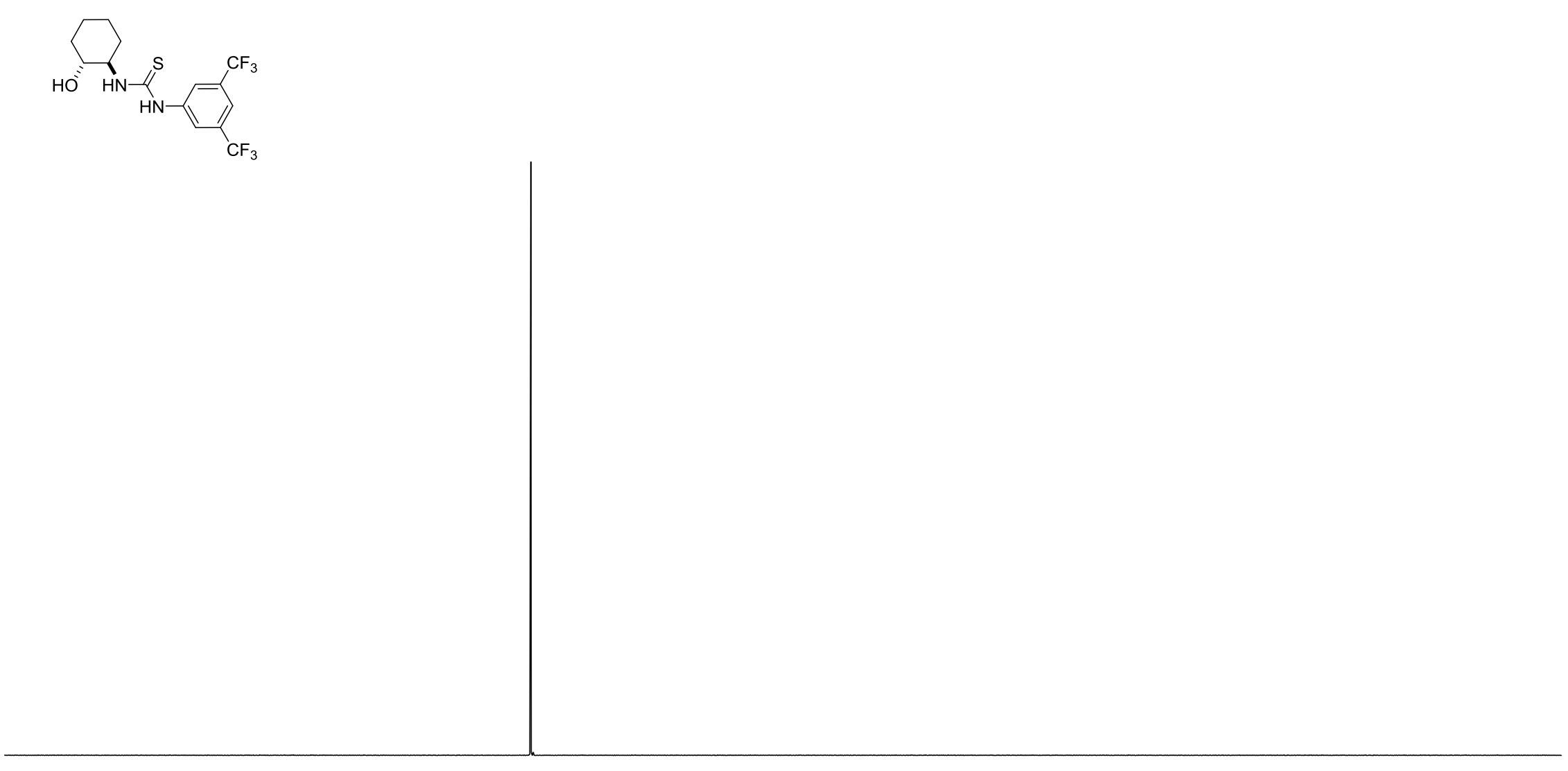




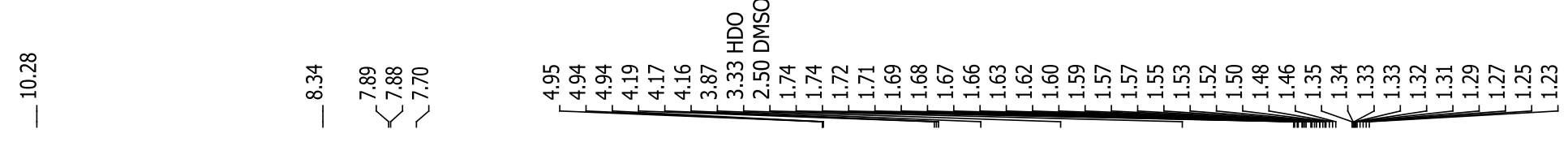

${ }^{1} \mathrm{H}-\mathrm{NMR}\left(600 \mathrm{MHz},\left(\mathrm{CD}_{3}\right)_{2} \mathrm{SO}\right)$ of $\mathbf{S 5 C}$
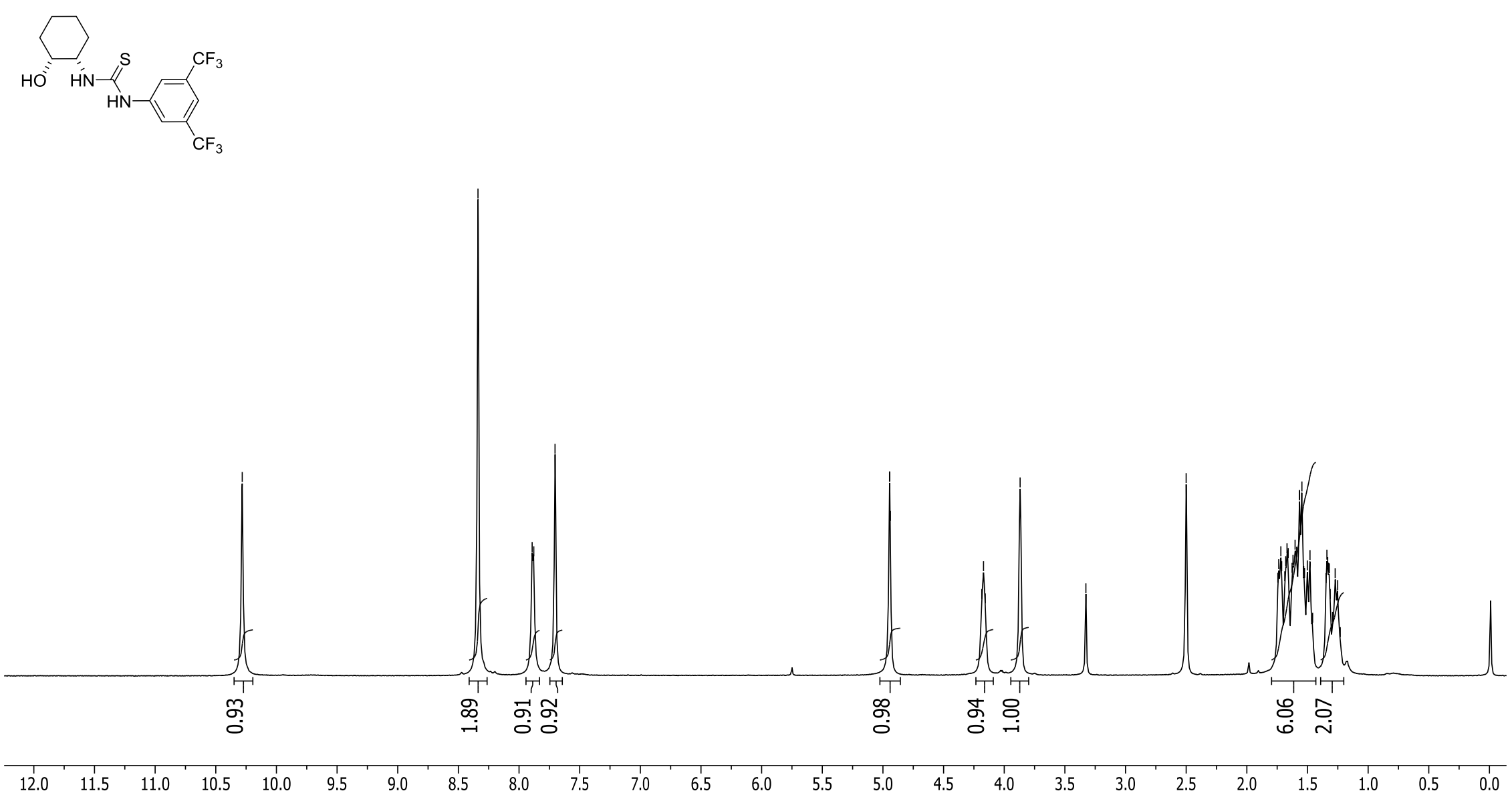


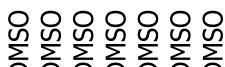

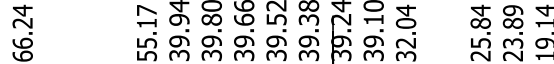

${ }^{13} \mathrm{C}-\mathrm{NMR}\left(150 \mathrm{MHz},\left(\mathrm{CD}_{3}\right)_{2} \mathrm{SO}\right)$ of $\mathbf{S} 5 \mathbf{c}$

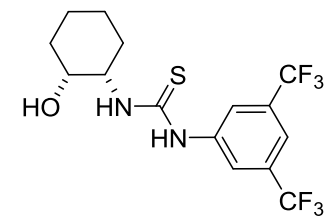

(N)

(n)

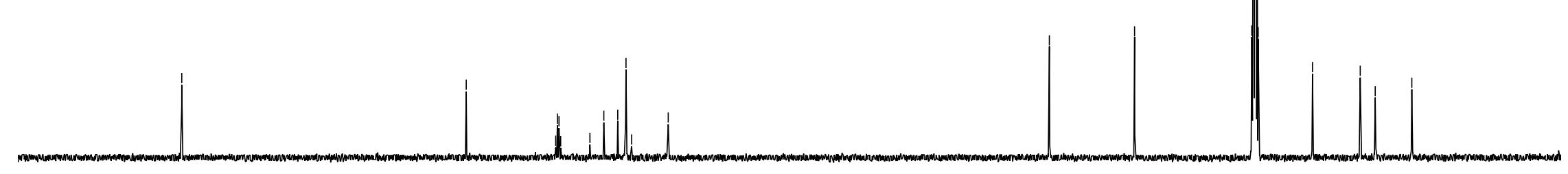

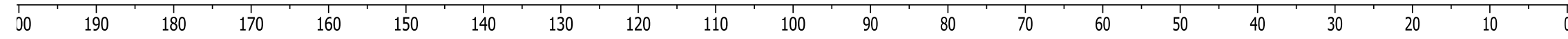


${ }^{19} \mathrm{~F}-\mathrm{NMR}\left(565 \mathrm{MHz},\left(\mathrm{CD}_{3}\right)_{2} \mathrm{SO}\right)$ of $\mathbf{S} 5 \mathrm{c}$
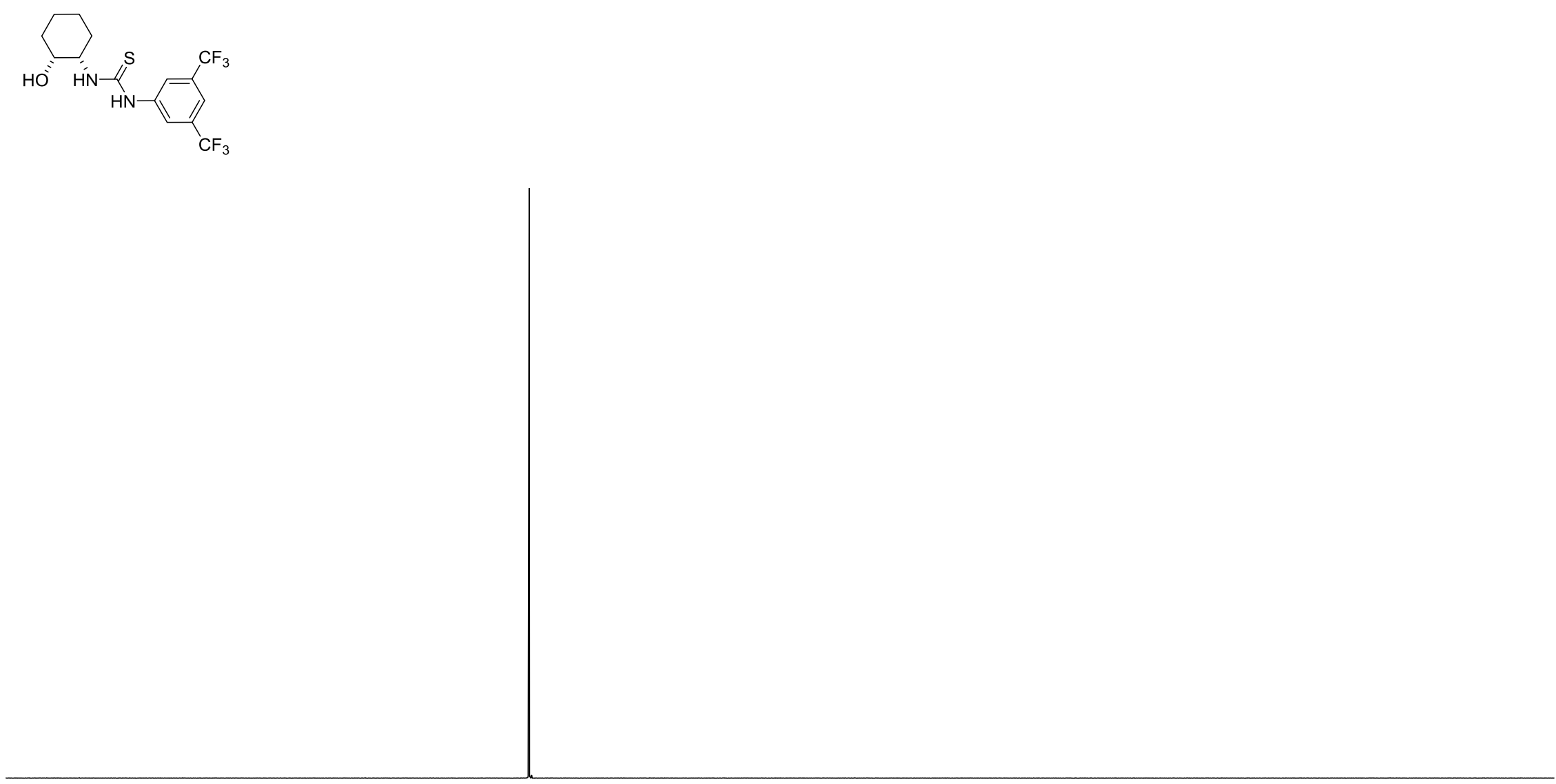

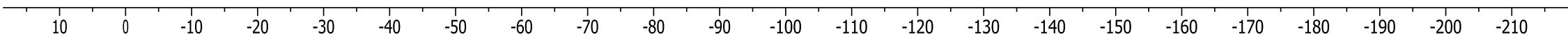


${ }^{1} \mathrm{H}-\mathrm{NMR}\left(600 \mathrm{MHz},\left(\mathrm{CD}_{3}\right)_{2} \mathrm{SO}\right)$ of $\mathbf{S} 5 \mathrm{~d}$
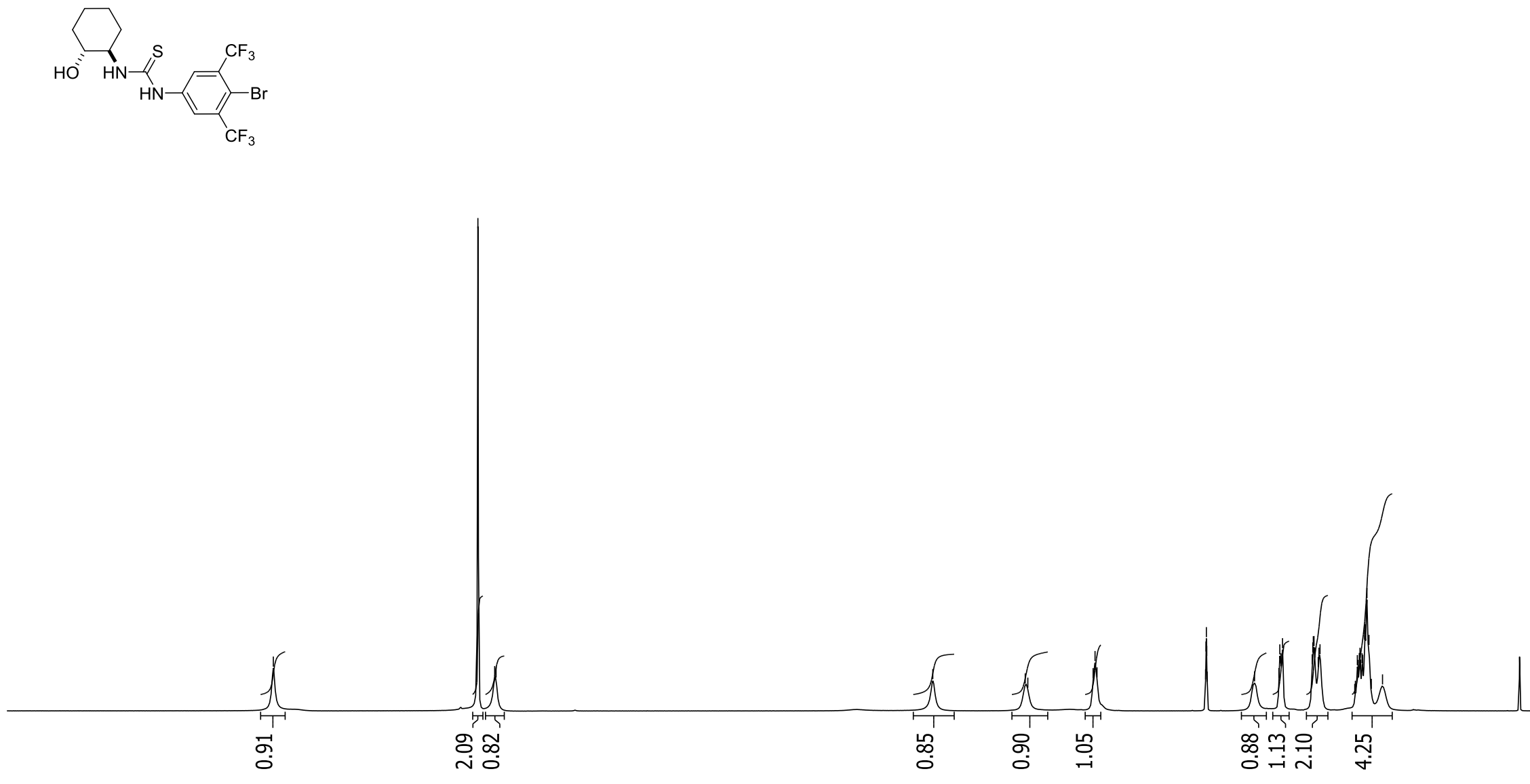

$\begin{array}{lllll}12.0 & 11.5 & 11.0 & 10.5 & 10.0\end{array}$

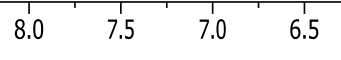
6.0 
${ }^{13} \mathrm{C}-\mathrm{NMR}\left(150 \mathrm{MHz},\left(\mathrm{CD}_{3}\right)_{2} \mathrm{SO}\right)$ of S5d

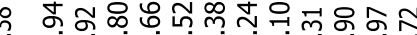

๙

I
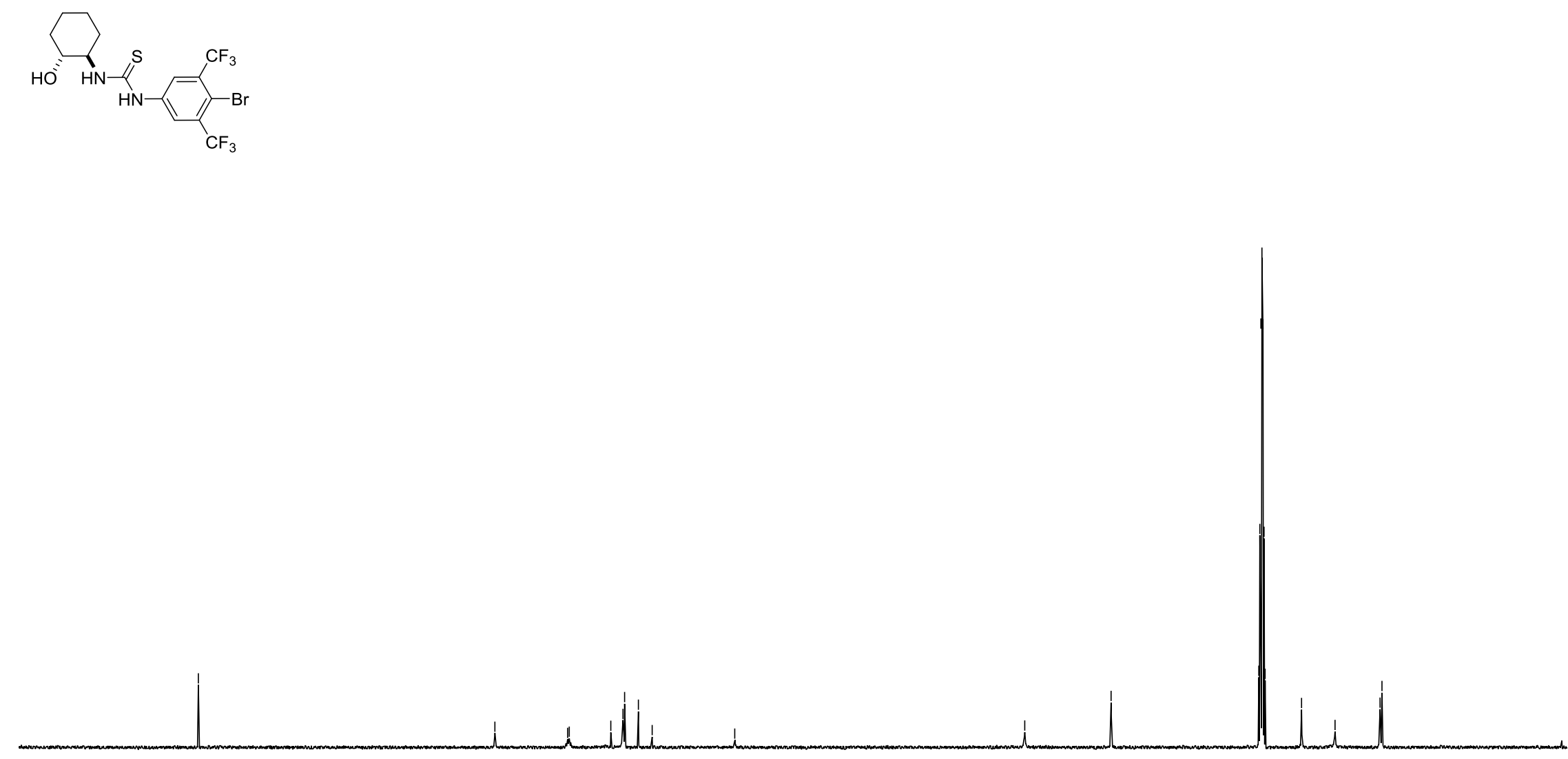

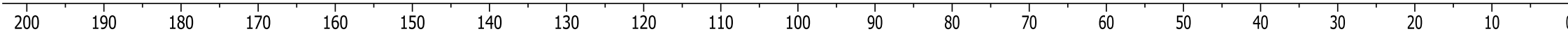


${ }^{19}$ F-NMR $\left(565 \mathrm{MHz},\left(\mathrm{CD}_{3}\right)_{2} \mathrm{SO}\right)$ of $\mathbf{S} 5 \mathbf{d}$
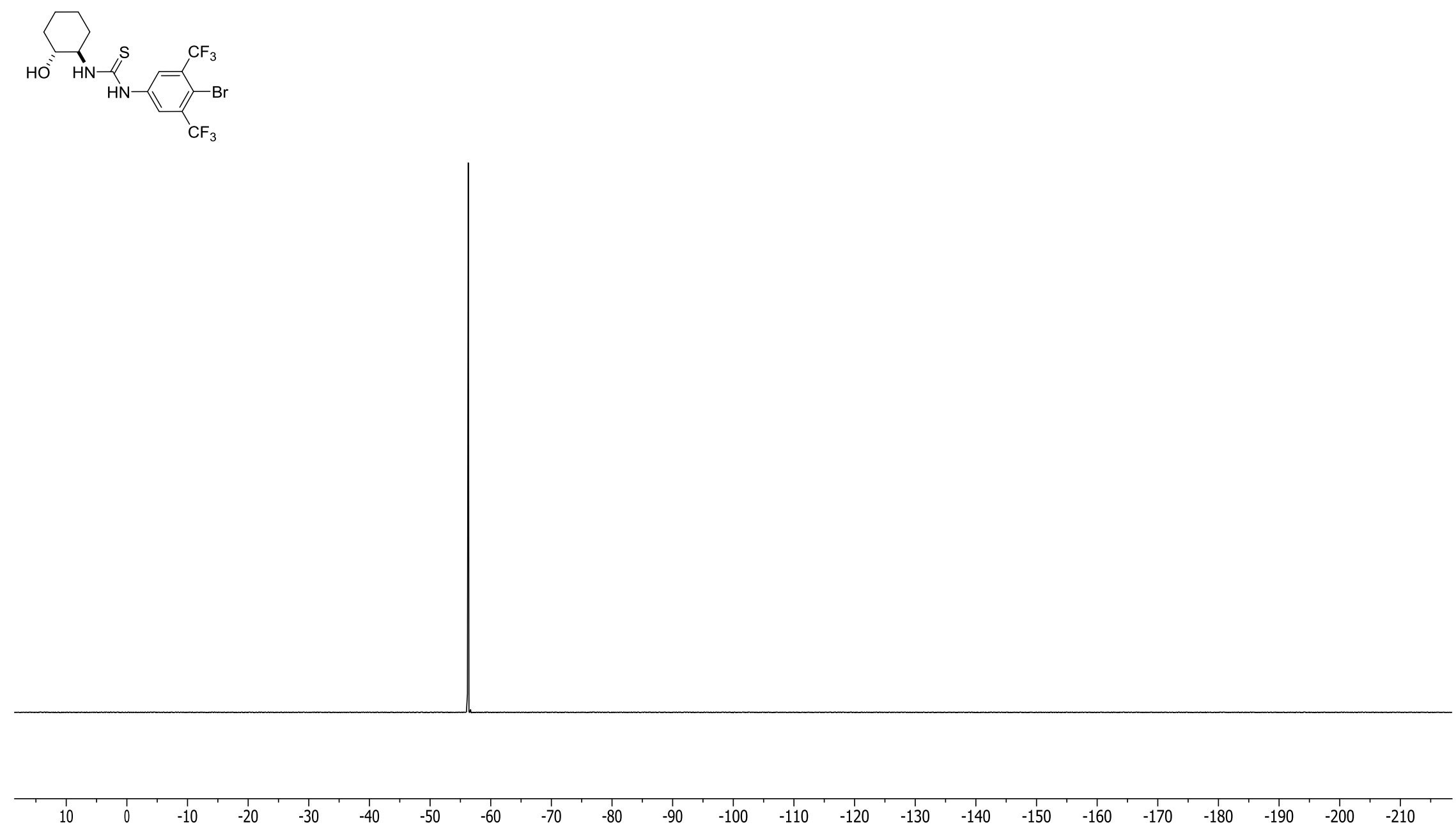


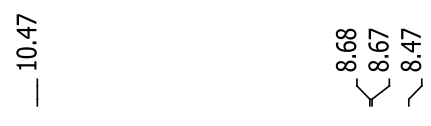

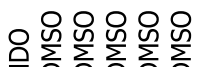

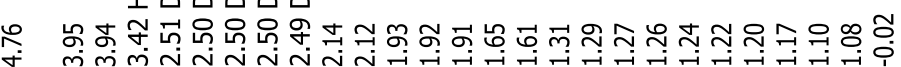

${ }^{1} \mathrm{H}-\mathrm{NMR}\left(600 \mathrm{MHz},\left(\mathrm{CD}_{3}\right)_{2} \mathrm{SO}\right)$ of $\mathbf{S} 5 \mathbf{e}$
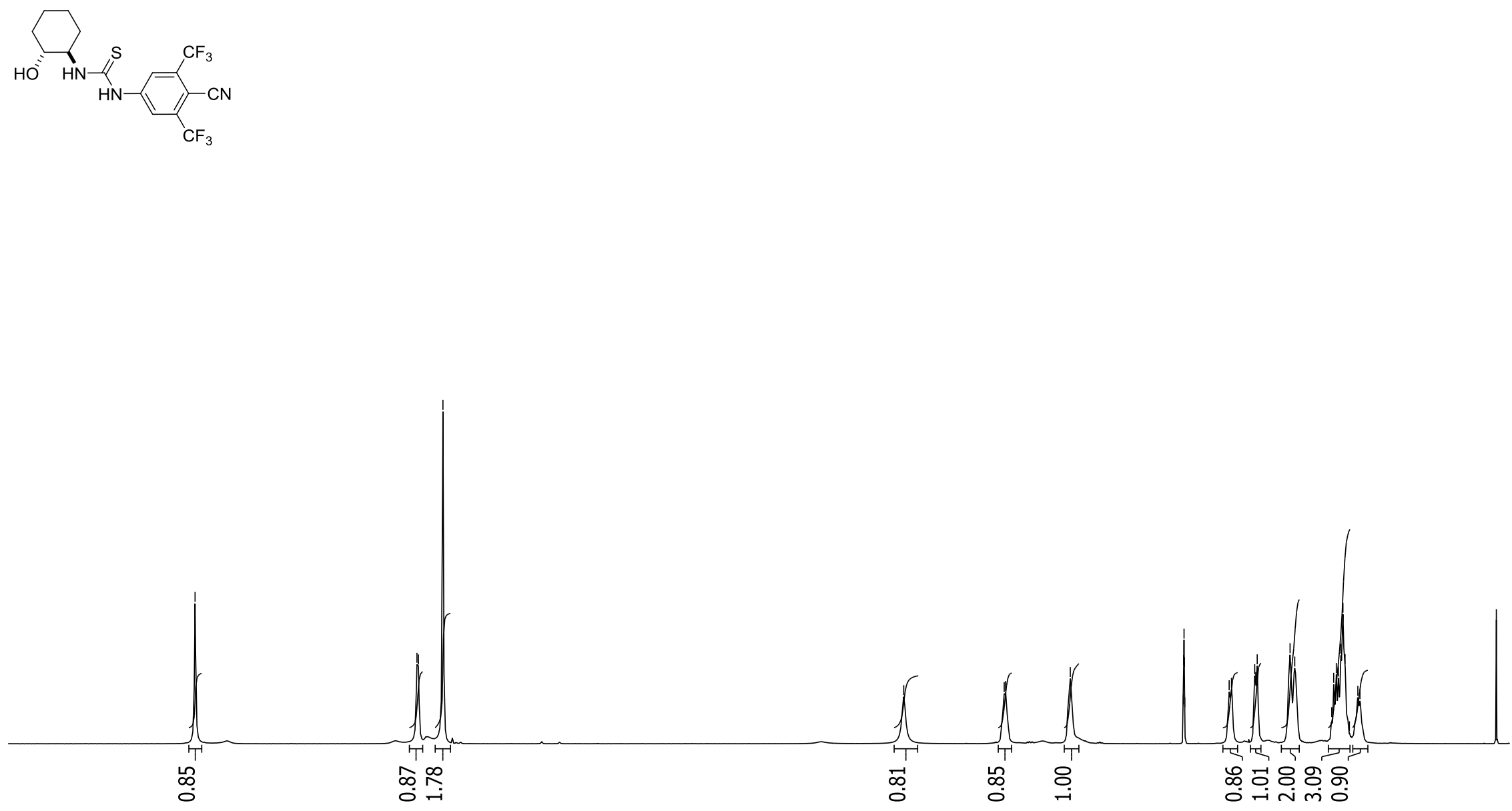

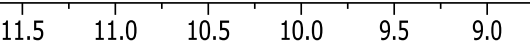
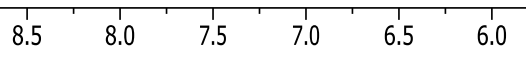


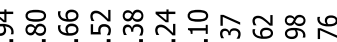

लं

(1)

${ }^{13} \mathrm{C}-\mathrm{NMR}\left(150 \mathrm{MHz},\left(\mathrm{CD}_{3}\right)_{2} \mathrm{SO}\right)$ of $\mathbf{S} 5 \mathbf{e}$
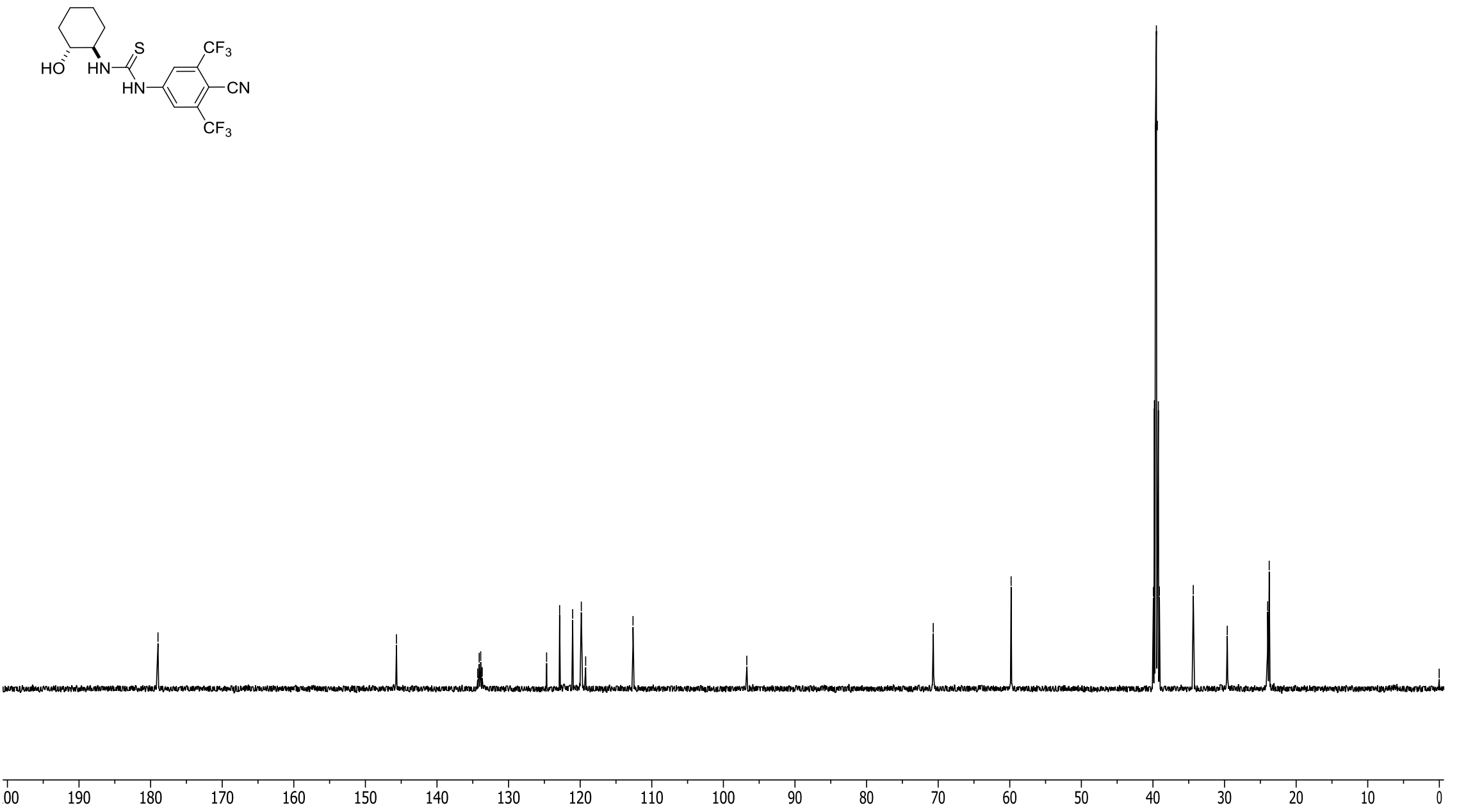
${ }^{19} \mathrm{~F}-\mathrm{NMR}\left(565 \mathrm{MHz},\left(\mathrm{CD}_{3}\right)_{2} \mathrm{SO}\right)$ of $\mathbf{S} 5 \mathbf{e}$
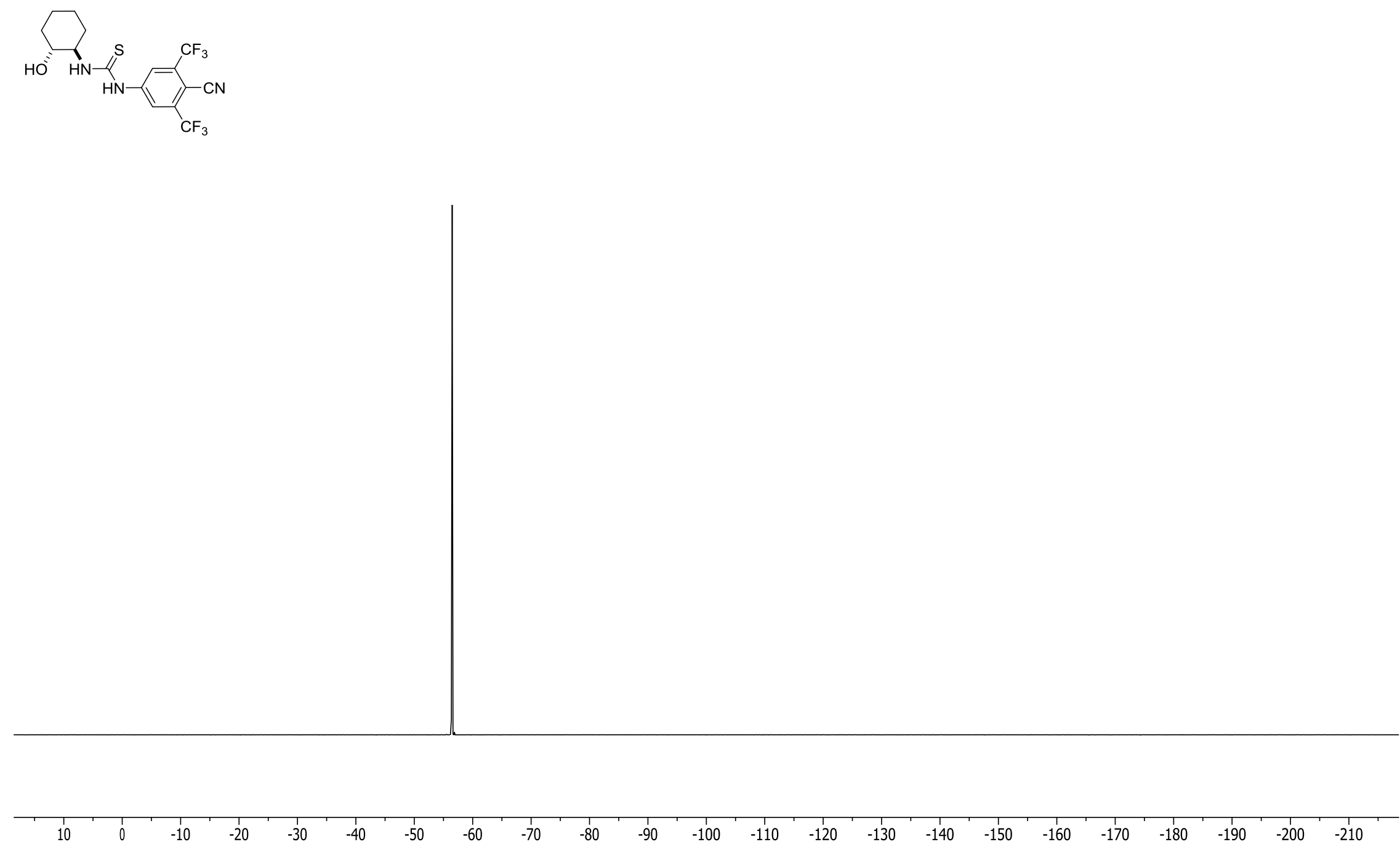


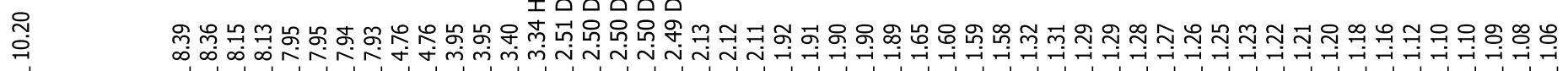

${ }^{1} \mathrm{H}-\mathrm{NMR}\left(600 \mathrm{MHz},\left(\mathrm{CD}_{3}\right)_{2} \mathrm{SO}\right)$ of $\mathbf{S 5 f}$
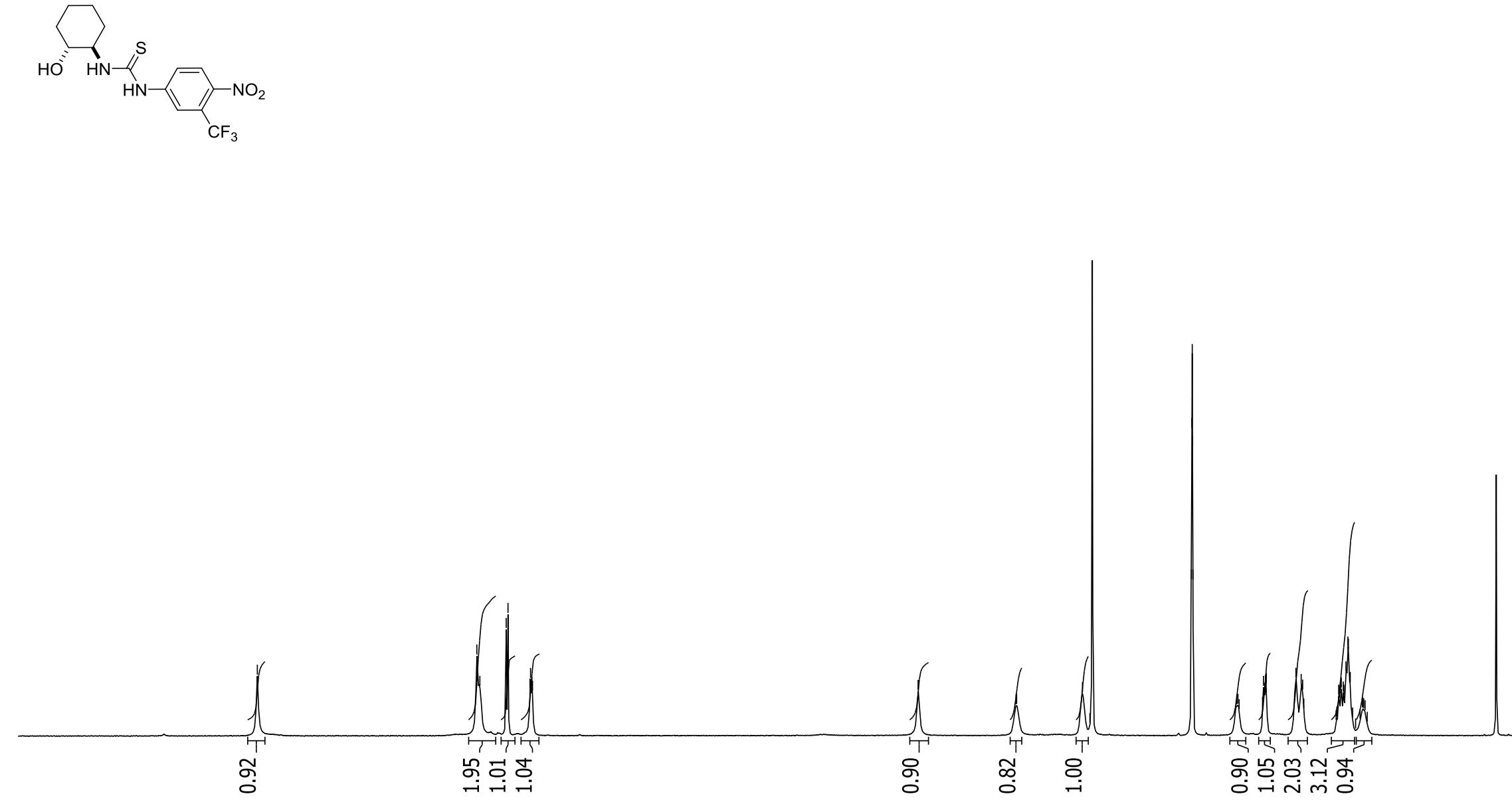

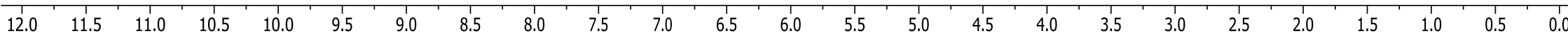



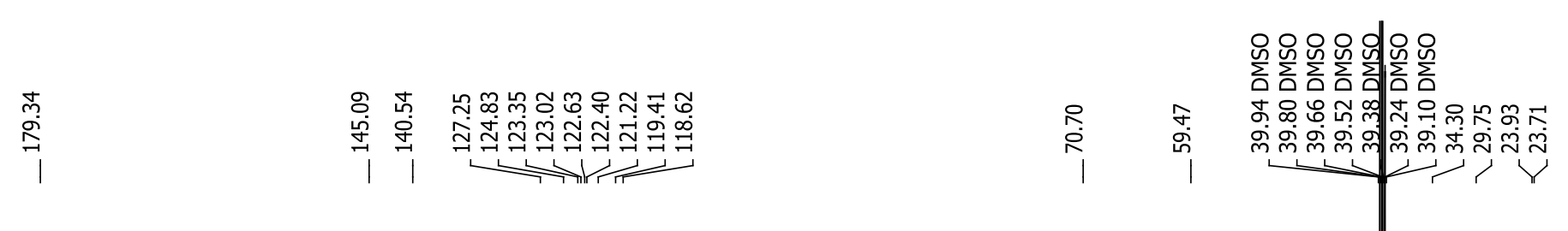

${ }^{13} \mathrm{C}-\mathrm{NMR}\left(150 \mathrm{MHz},\left(\mathrm{CD}_{3}\right)_{2} \mathrm{SO}\right)$ of $\mathbf{S} 5 \mathrm{f}$
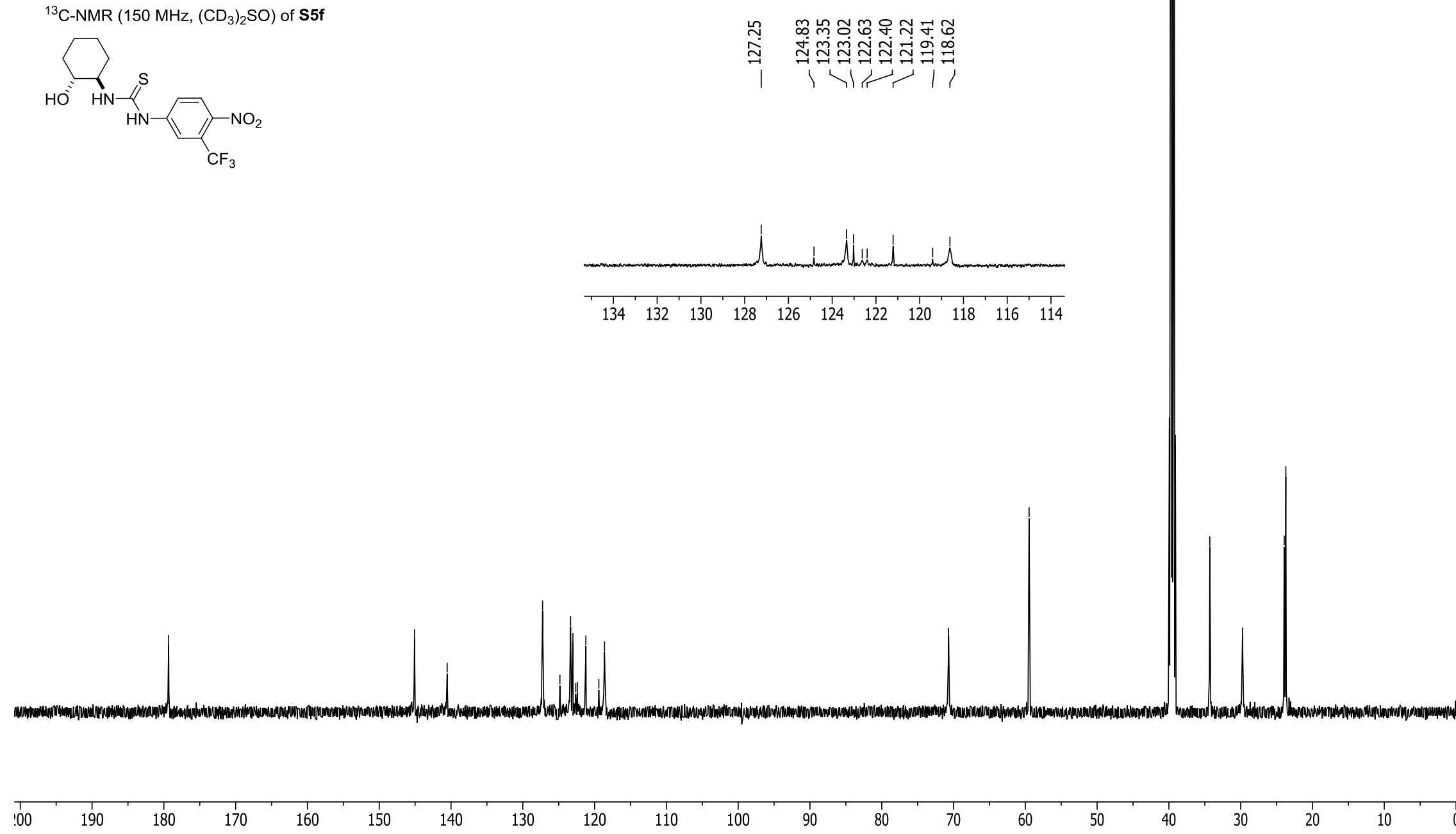
${ }^{19} \mathrm{~F}-\mathrm{NMR}\left(565 \mathrm{MHz},\left(\mathrm{CD}_{3}\right)_{2} \mathrm{SO}\right)$ of $\mathbf{S} 5 \mathbf{f}$
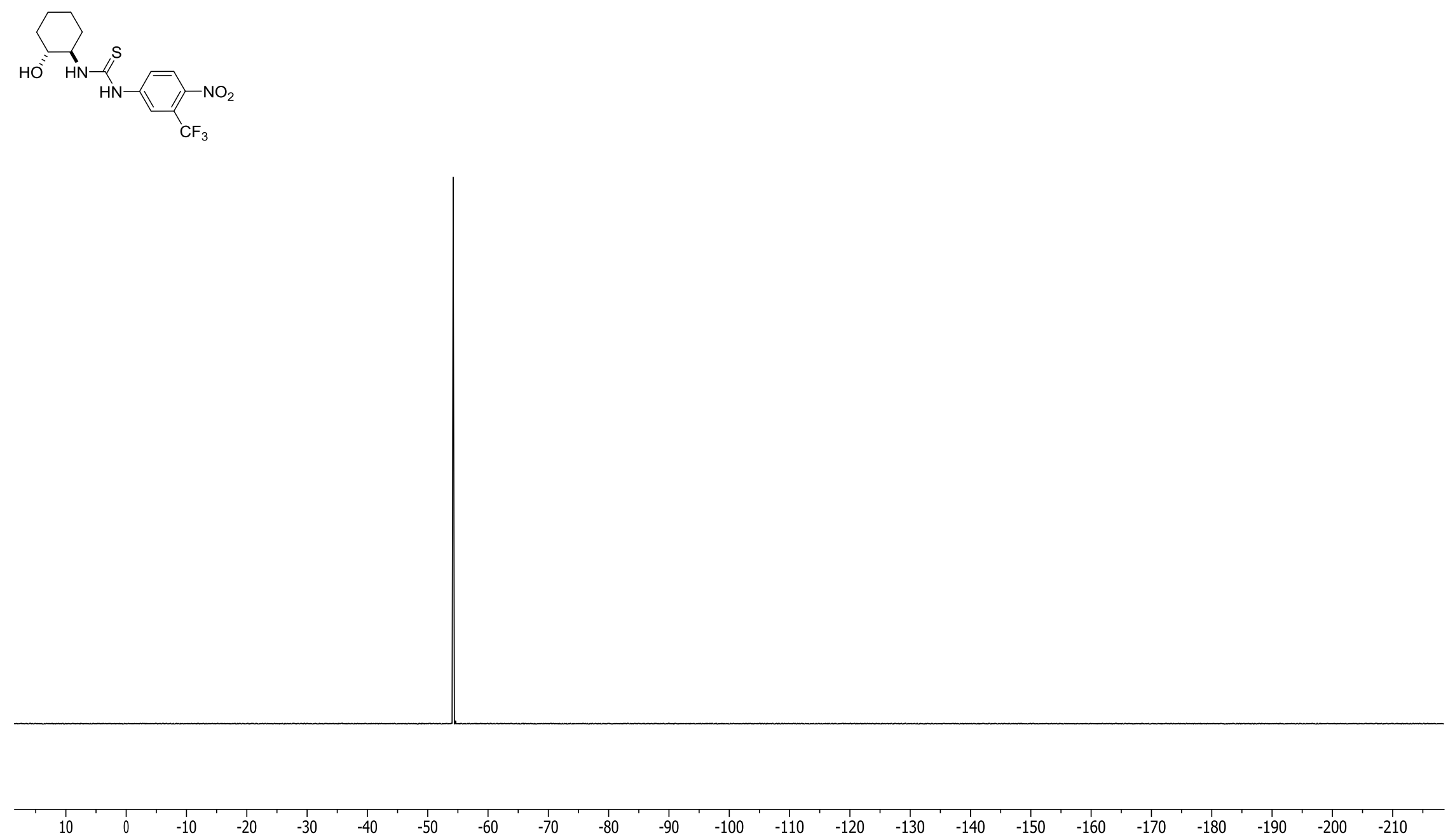


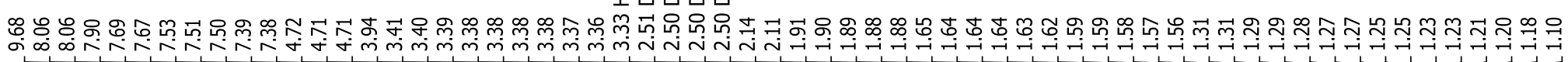

${ }^{1} \mathrm{H}-\mathrm{NMR}\left(600 \mathrm{MHz},\left(\mathrm{CD}_{3}\right)_{2} \mathrm{SO}\right)$ of $\mathbf{S} 5 \mathrm{~g}$
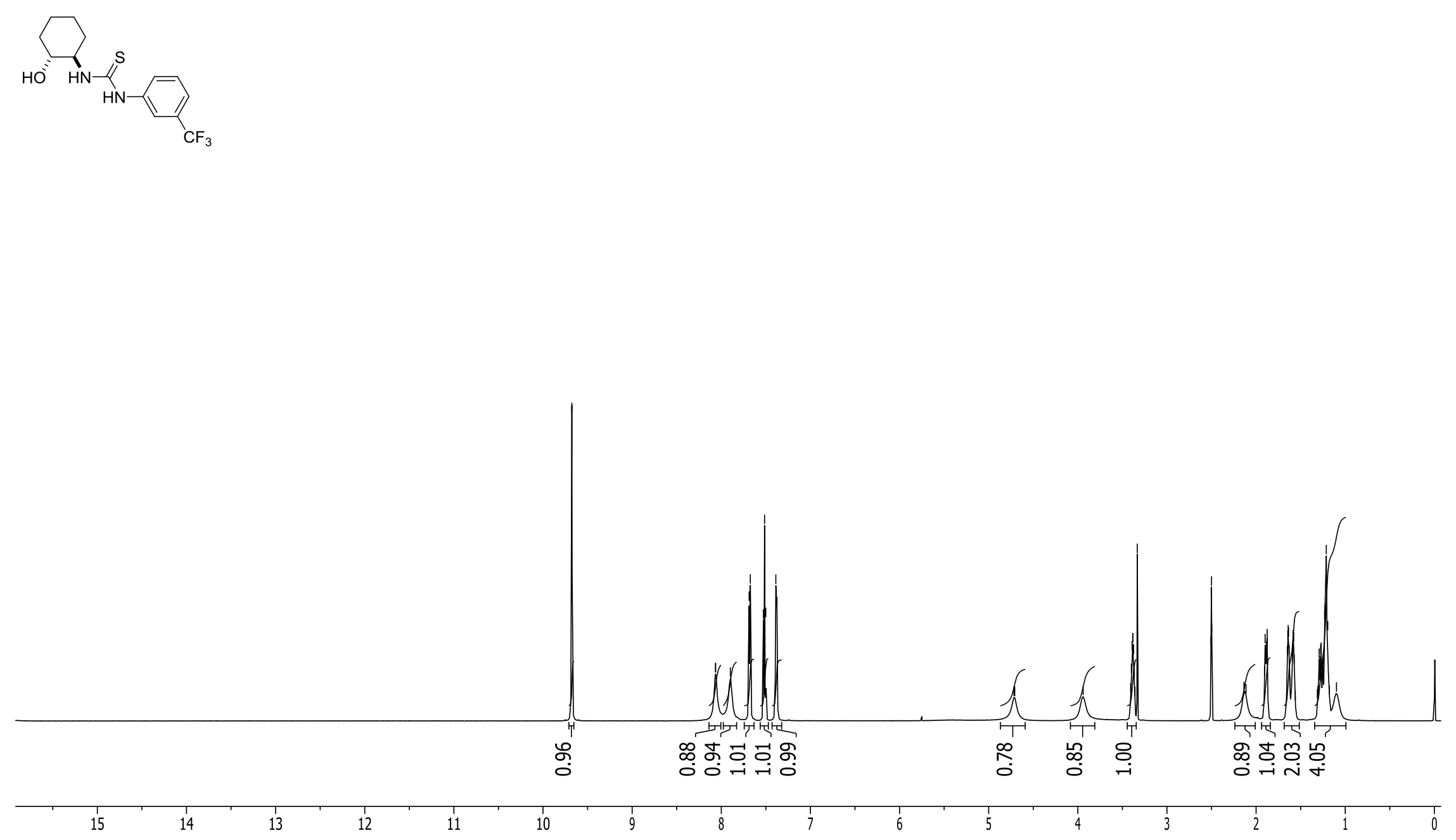

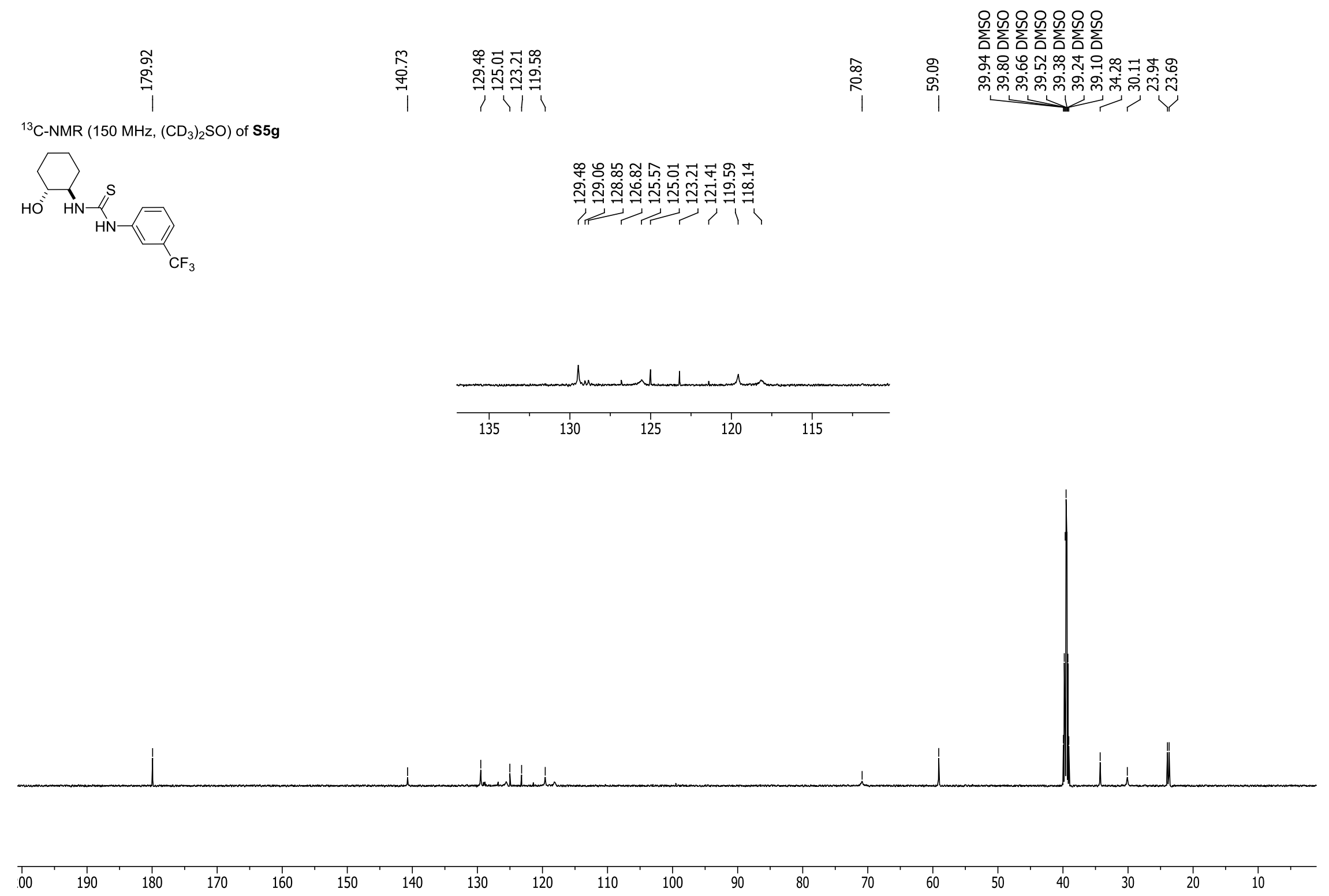


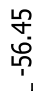

${ }^{19} \mathrm{~F}-\mathrm{NMR}\left(565 \mathrm{MHz},\left(\mathrm{CD}_{3}\right)_{2} \mathrm{SO}\right)$ of $\mathbf{S} 5 \mathrm{~g}$
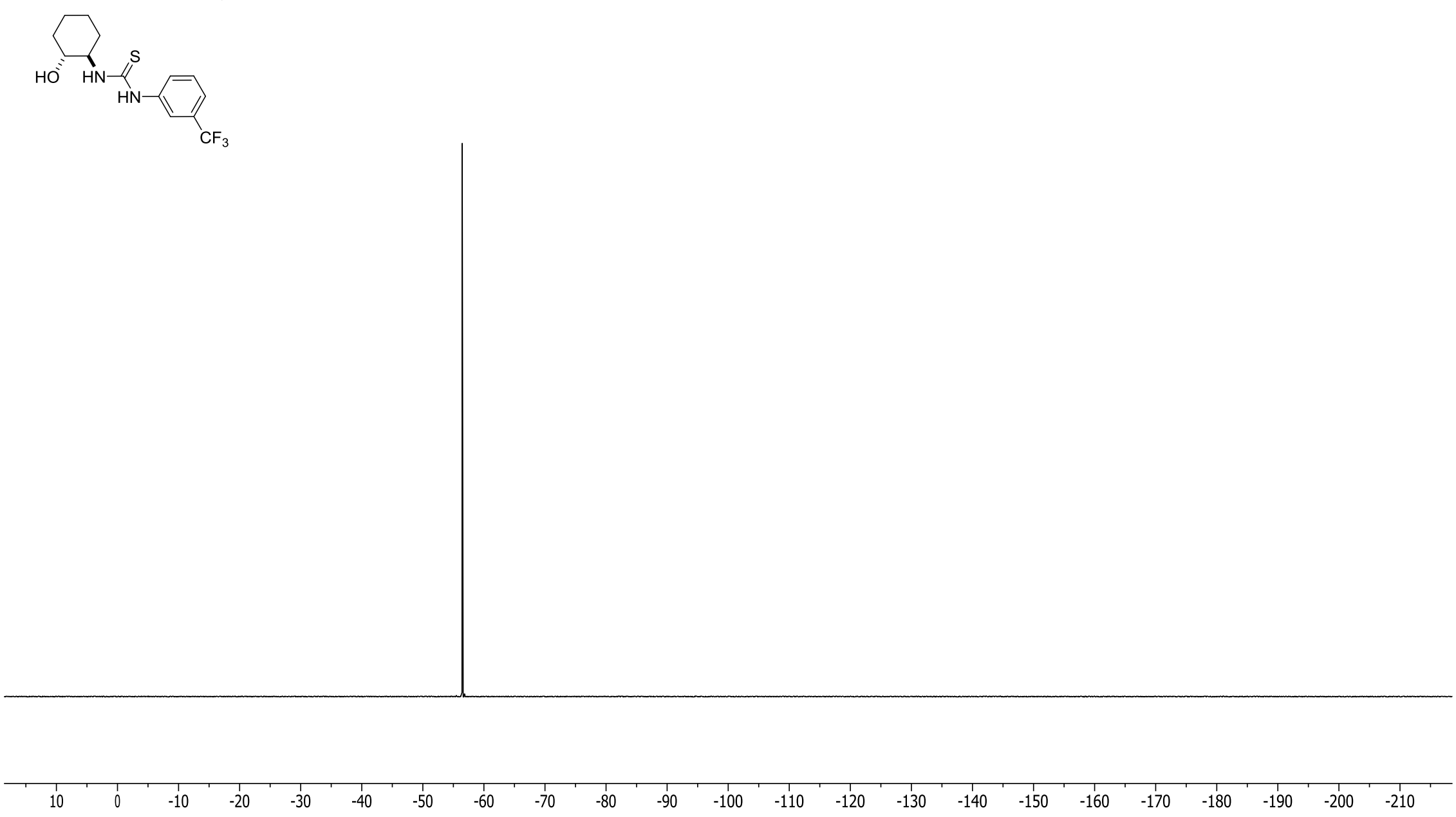
${ }^{1} \mathrm{H}-\mathrm{NMR}\left(600 \mathrm{MHz},\left(\mathrm{CD}_{3}\right)_{2} \mathrm{SO}\right)$ of $\mathbf{4 b}$
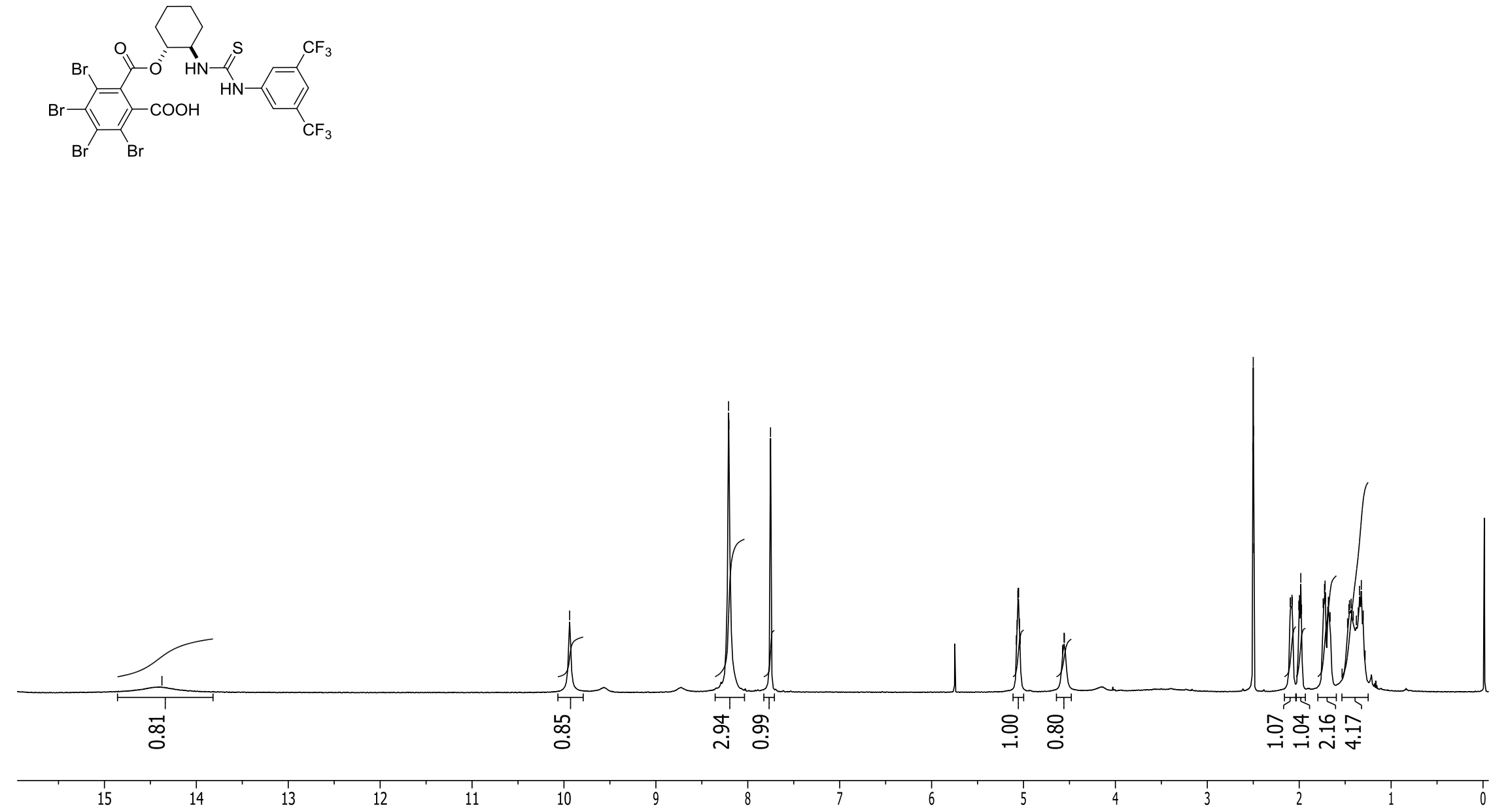


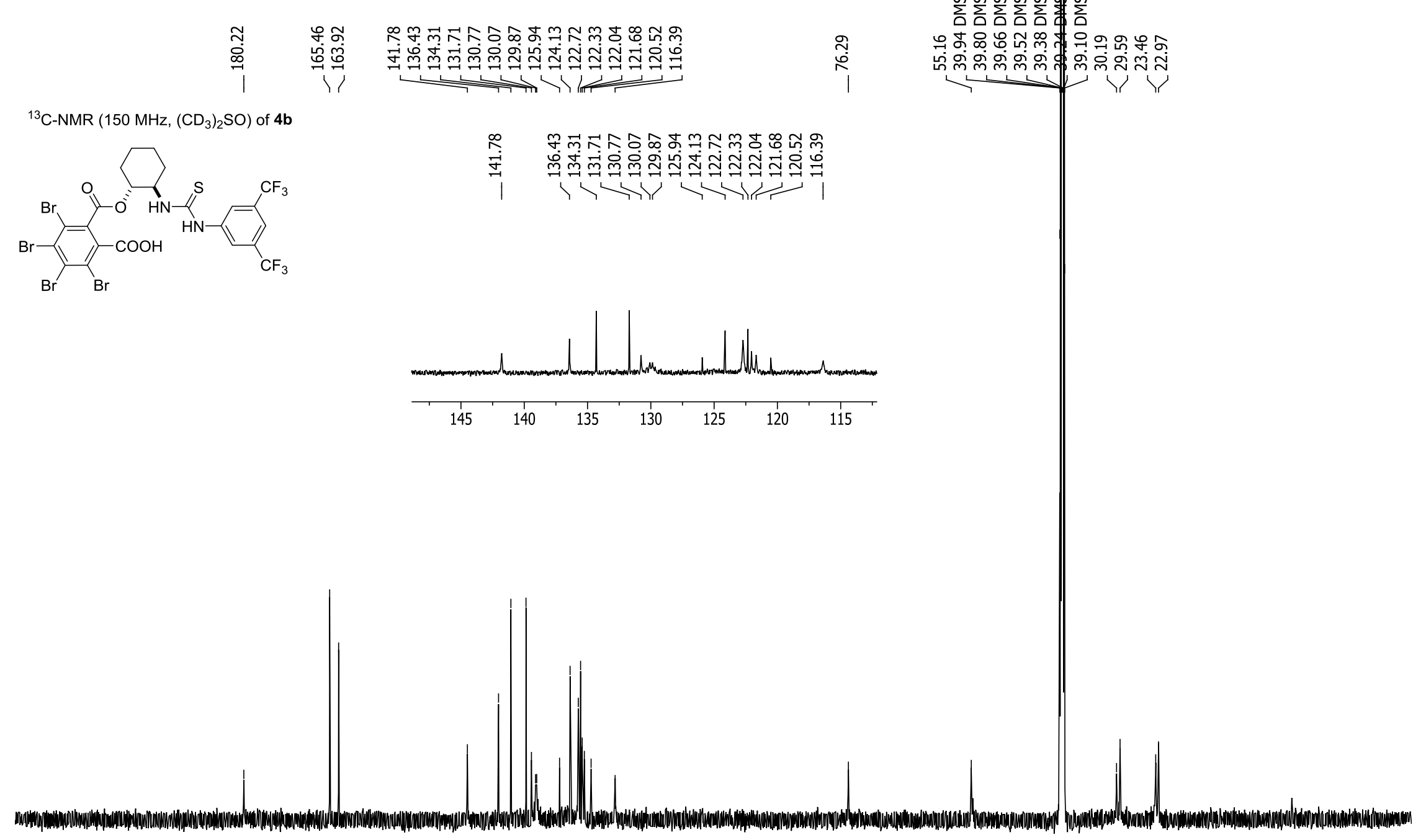

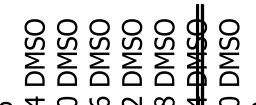

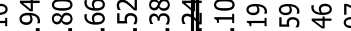

ம்

${ }^{13} \mathrm{C}-\mathrm{NMR}\left(150 \mathrm{MHz},\left(\mathrm{CD}_{3}\right)_{2} \mathrm{SO}\right)$ of $4 \mathrm{~b}$

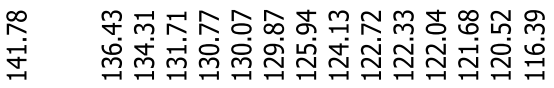

पो

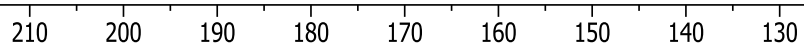

$120 \quad 110 \quad 100$

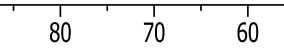

$50 \quad 40$

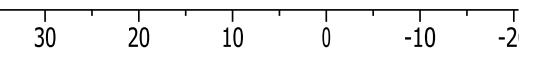


${ }^{19} \mathrm{~F}-\mathrm{NMR}\left(565 \mathrm{MHz},\left(\mathrm{CD}_{3}\right)_{2} \mathrm{SO}\right)$ of $4 \mathrm{~b}$
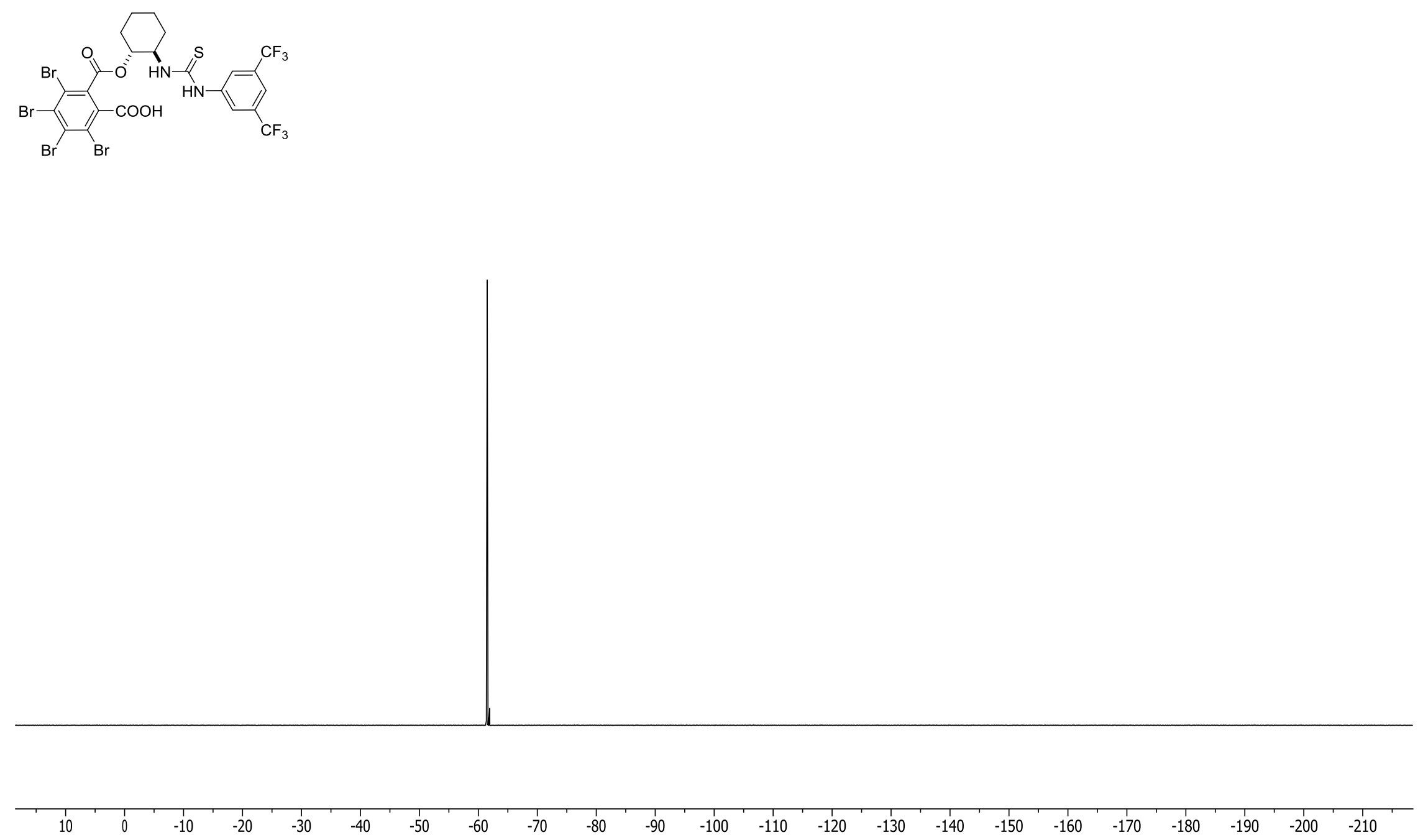


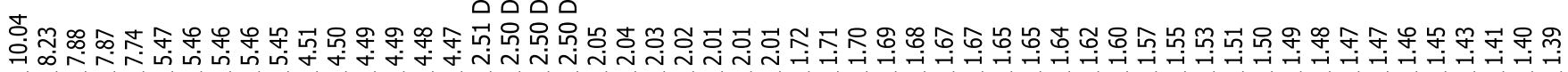

${ }^{1} \mathrm{H}-\mathrm{NMR}\left(600 \mathrm{MHz},\left(\mathrm{CD}_{3}\right)_{2} \mathrm{SO}\right)$ of $4 \mathrm{c}$
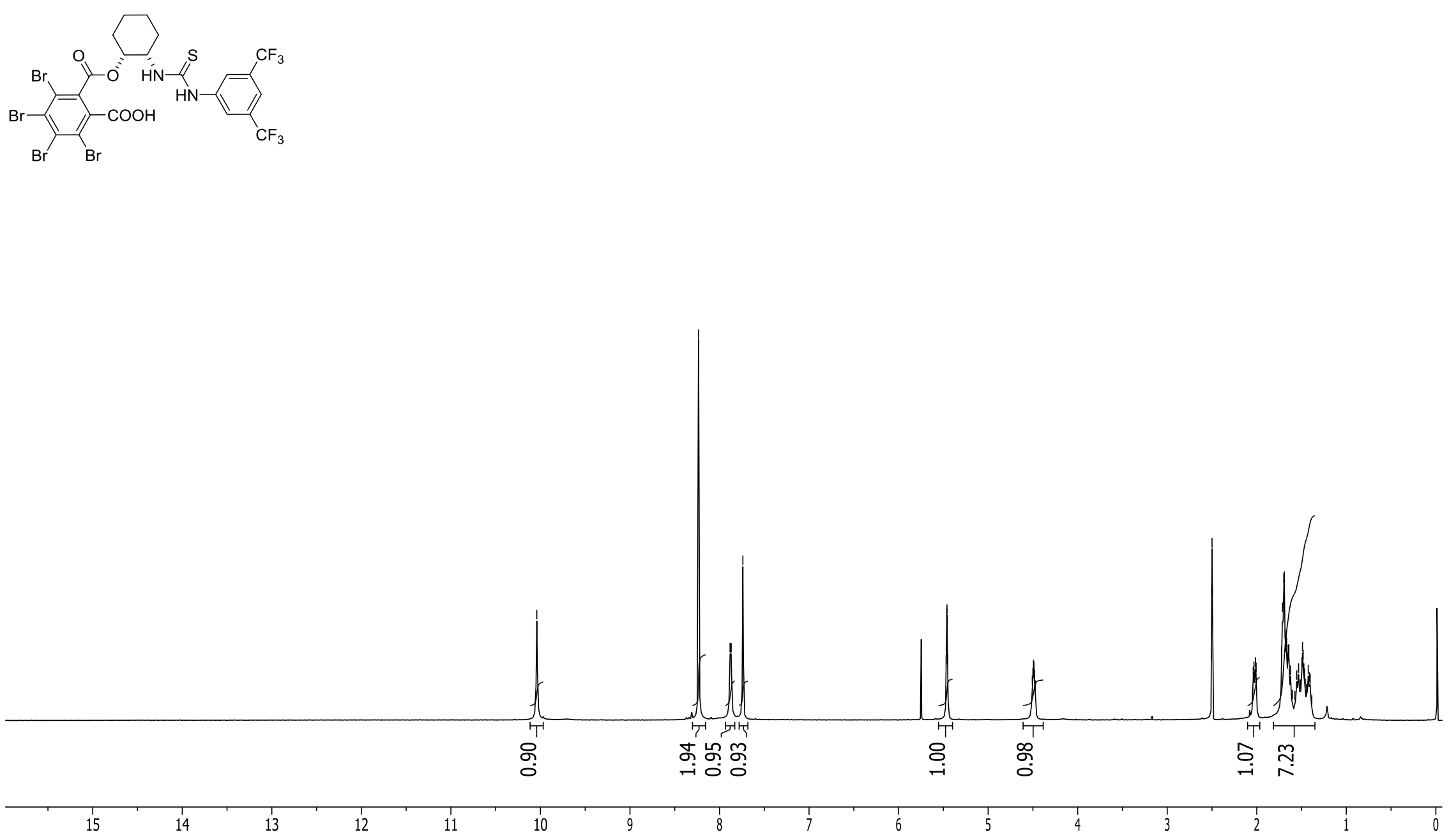


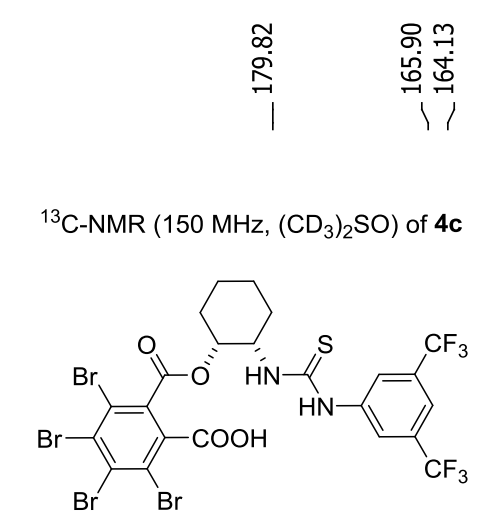

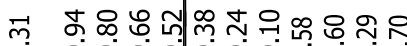

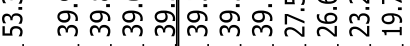

${ }^{13} \mathrm{C}-\mathrm{NMR}\left(150 \mathrm{MHz},\left(\mathrm{CD}_{3}\right)_{2} \mathrm{SO}\right)$ of $4 \mathrm{c}$

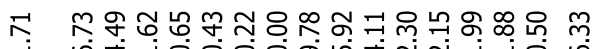

咅

|

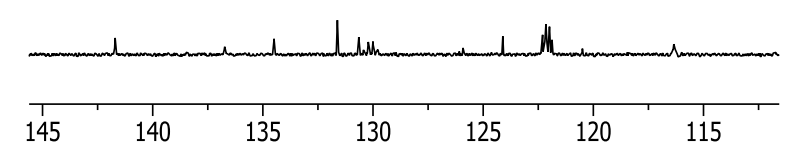

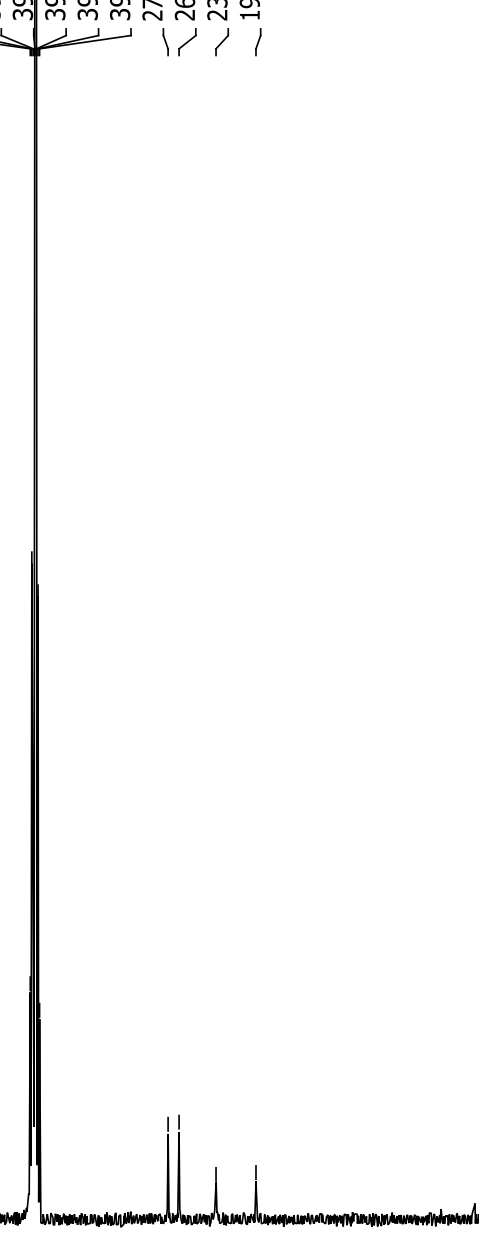


${ }^{19} \mathrm{~F}-\mathrm{NMR}\left(565 \mathrm{MHz},\left(\mathrm{CD}_{3}\right)_{2} \mathrm{SO}\right)$ of $4 \mathrm{c}$
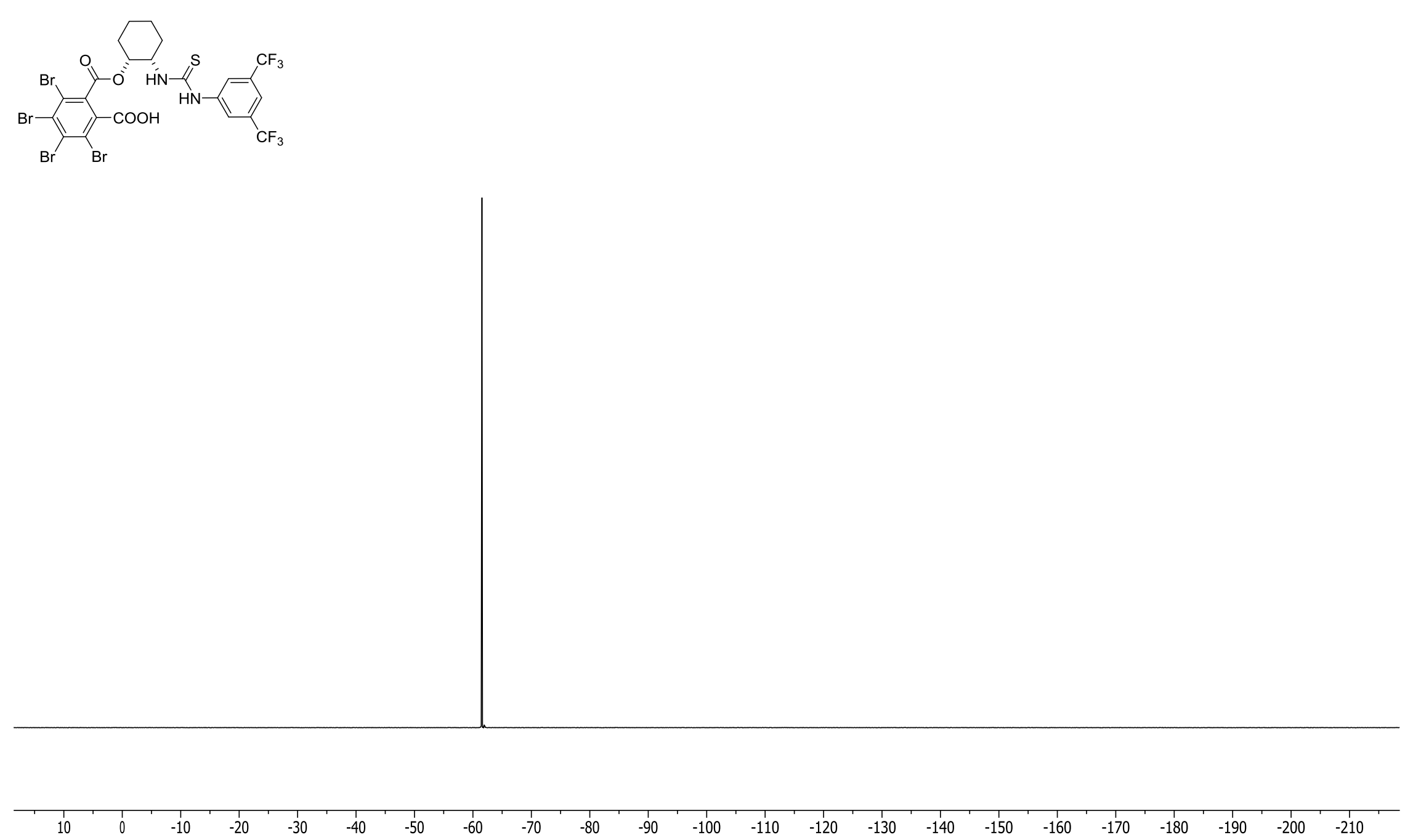
${ }^{1} \mathrm{H}-\mathrm{NMR}\left(600 \mathrm{MHz},\left(\mathrm{CD}_{3}\right)_{2} \mathrm{SO}\right)$ of $4 \mathrm{~d}$

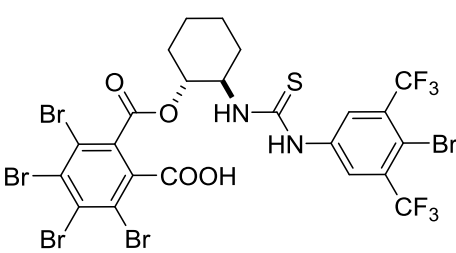

Niniti-ititi

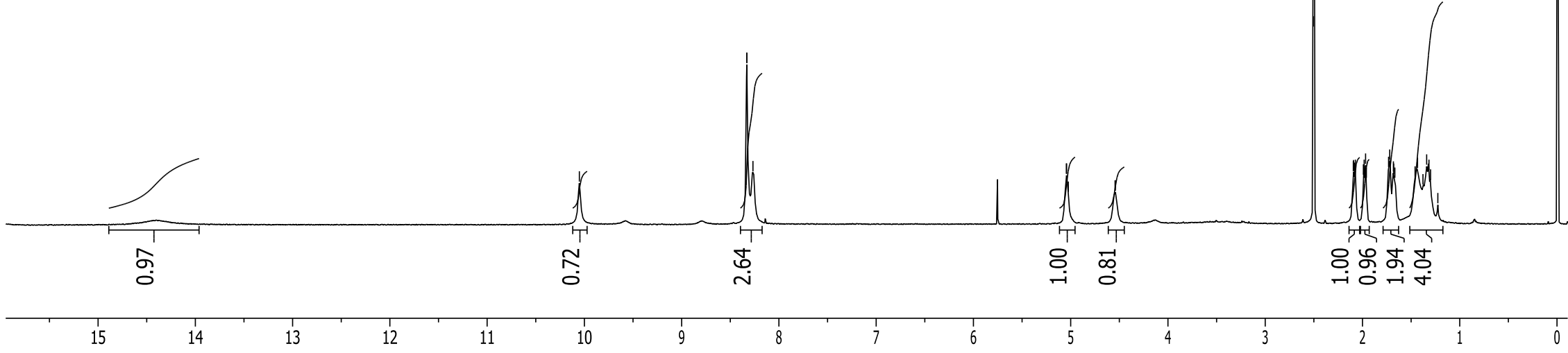




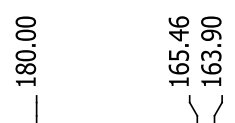

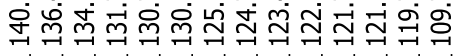

${ }^{13} \mathrm{C}-\mathrm{NMR}\left(150 \mathrm{MHz},\left(\mathrm{CD}_{3}\right)_{2} \mathrm{SO}\right)$ of $4 \mathrm{~d}$

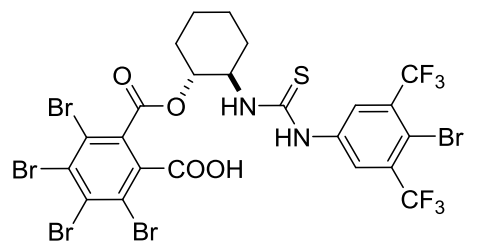

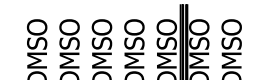

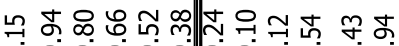

นं
旁

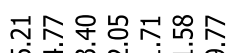

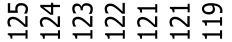

औ। 11

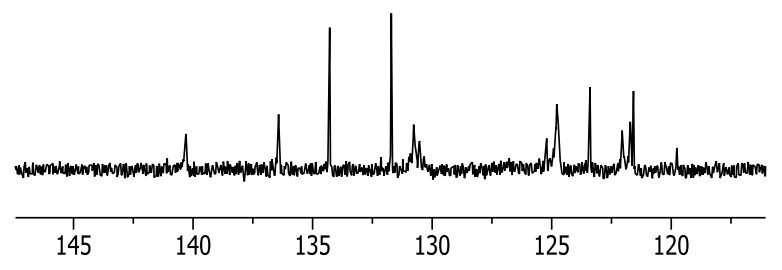

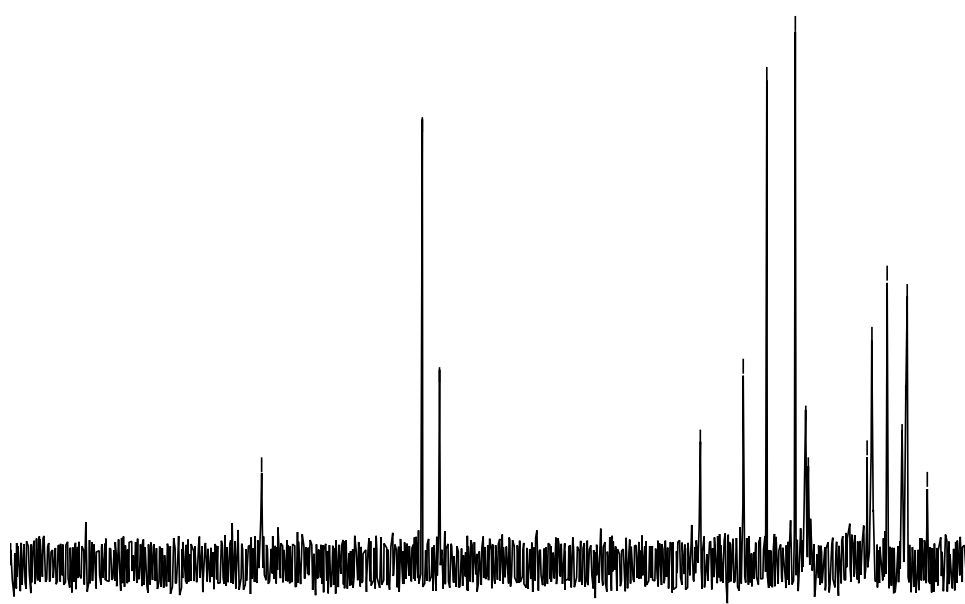

200

$190 \quad 180$

$170 \quad 160$

150

140

130

120

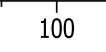

90

80

70

$60 \quad 50$

40 
${ }^{19} \mathrm{~F}-\mathrm{NMR}\left(565 \mathrm{MHz},\left(\mathrm{CD}_{3}\right)_{2} \mathrm{SO}\right)$ of $\mathbf{4 d}$
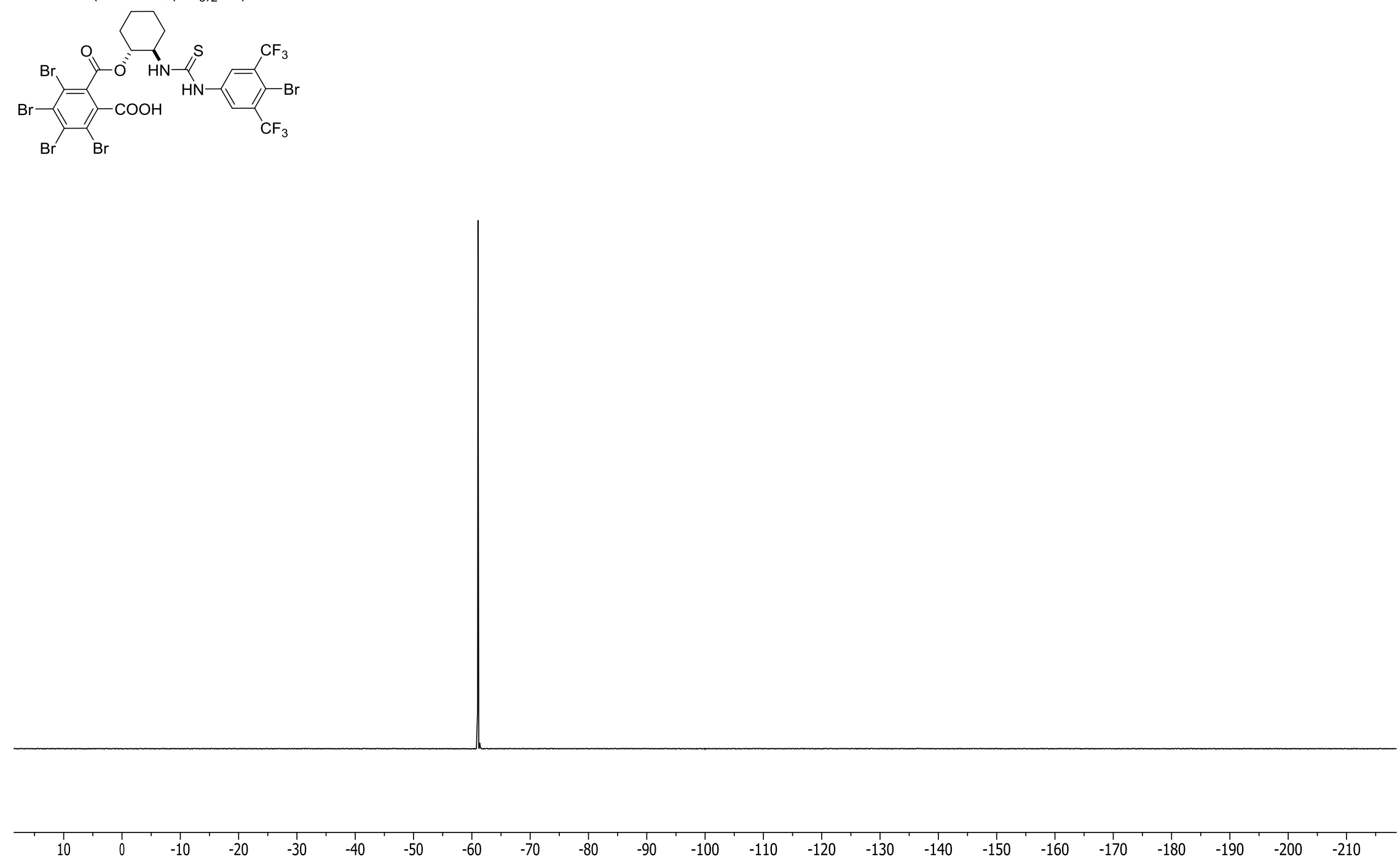


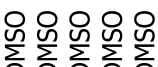

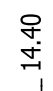

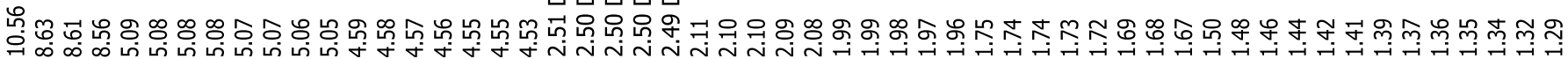

${ }^{1} \mathrm{H}-\mathrm{NMR}\left(600 \mathrm{MHz},\left(\mathrm{CD}_{3}\right)_{2} \mathrm{SO}\right)$ of $4 \mathbf{e}$
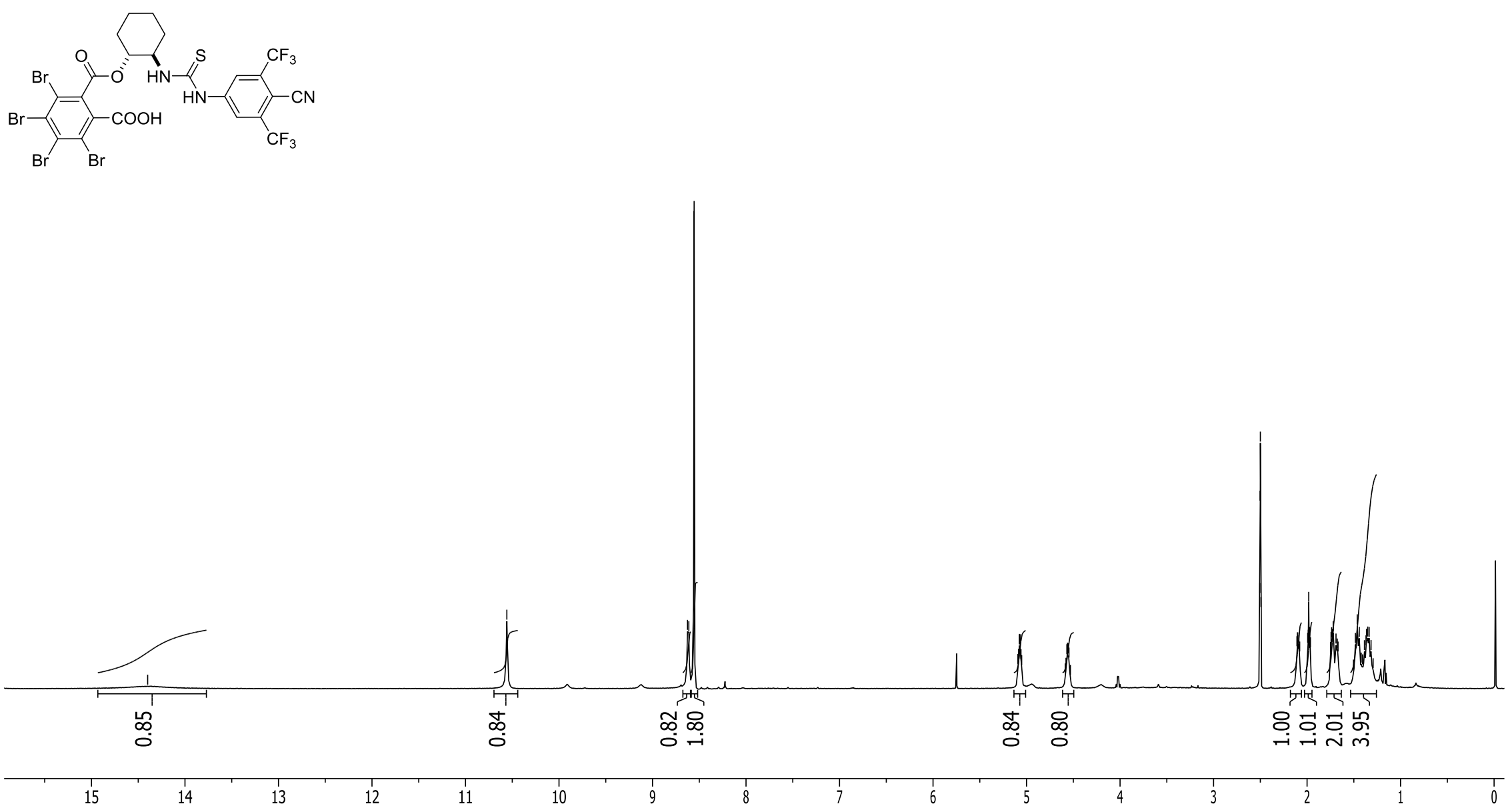

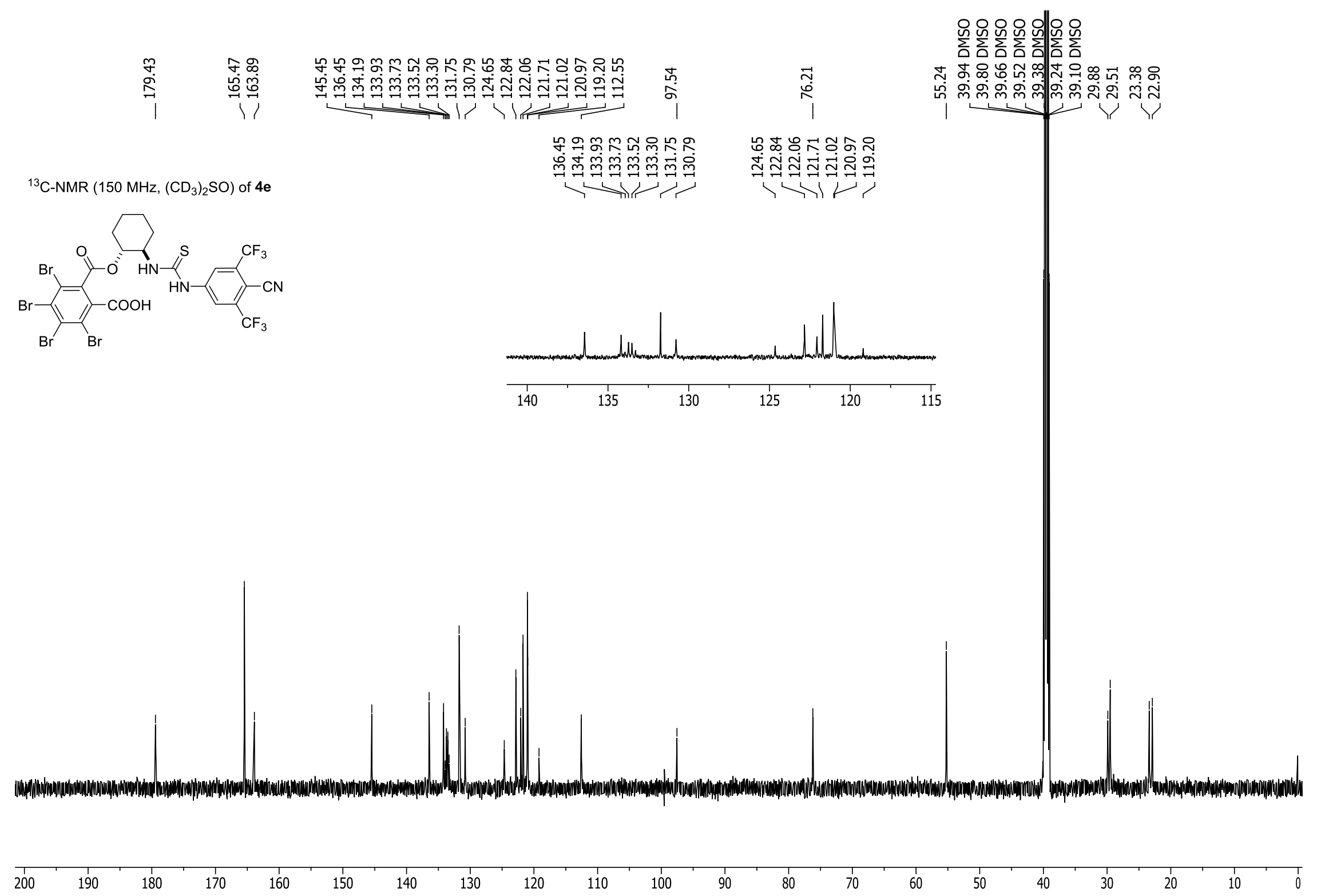
${ }^{19} \mathrm{~F}-\mathrm{NMR}\left(565 \mathrm{MHz},\left(\mathrm{CD}_{3}\right)_{2} \mathrm{SO}\right)$ of $4 \mathrm{e}$
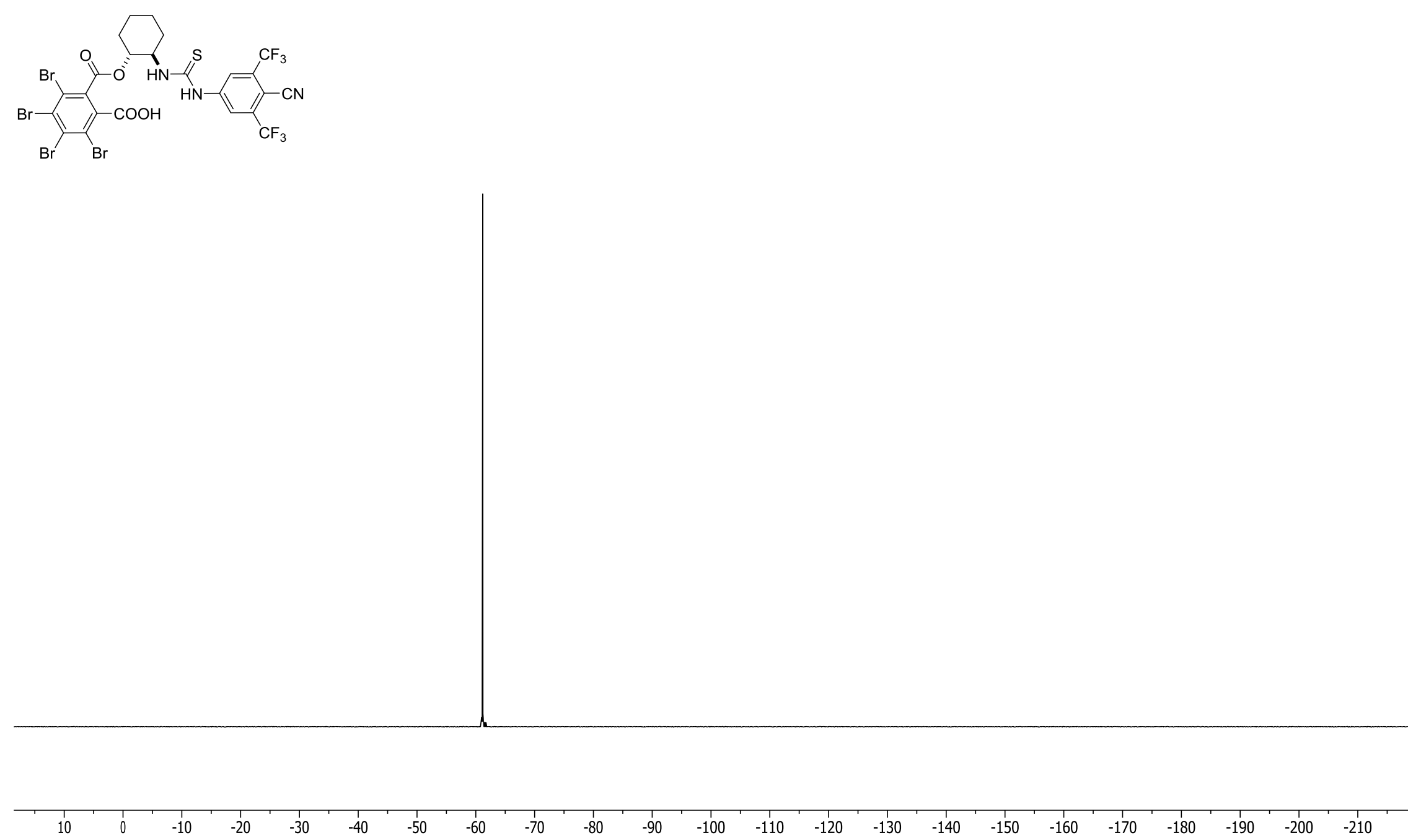


\section{${ }^{1} \mathrm{H}-\mathrm{NMR}\left(600 \mathrm{MHz},\left(\mathrm{CD}_{3}\right)_{2} \mathrm{SO}\right)$ of $\mathbf{4 f}$}
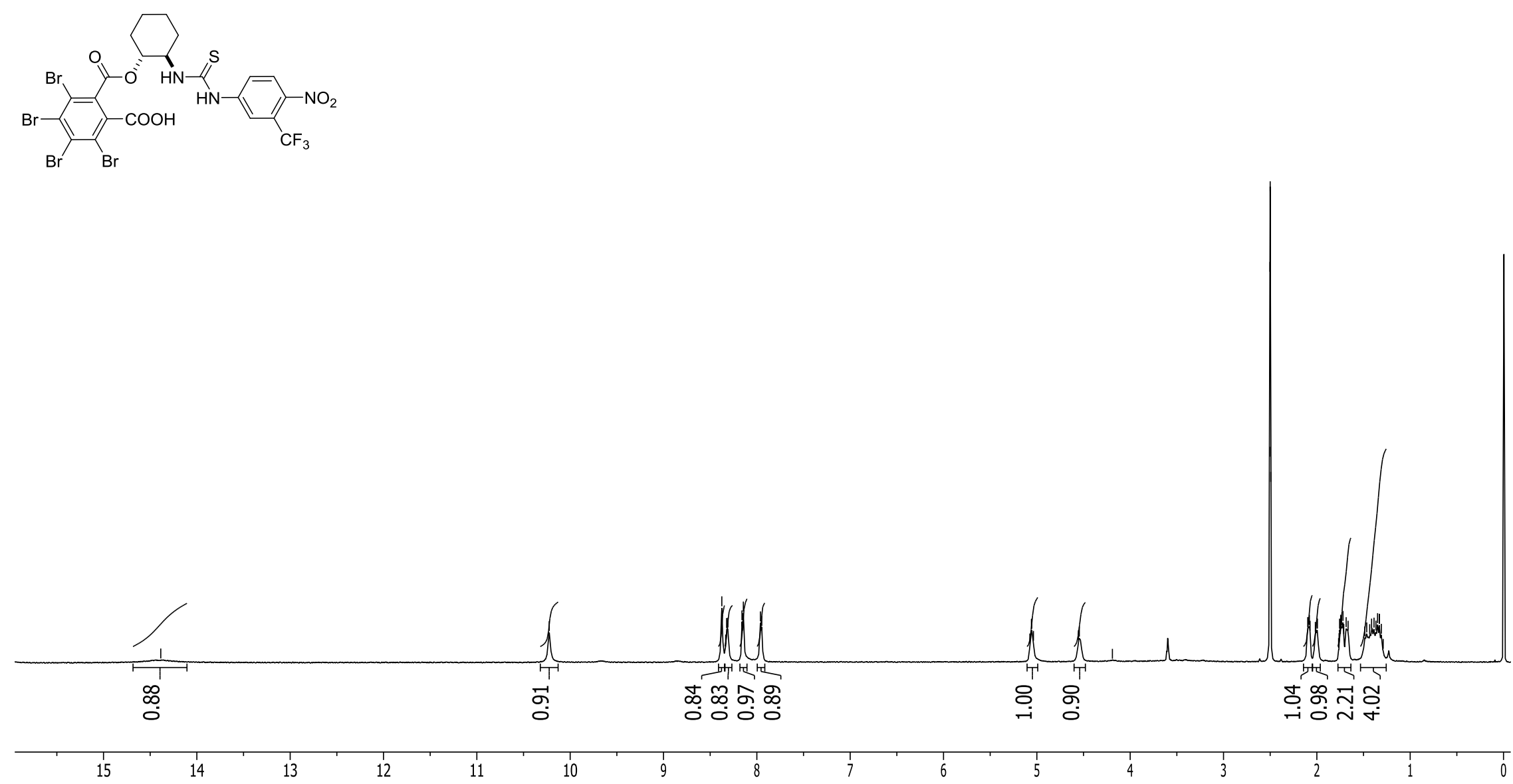


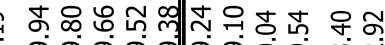

นี
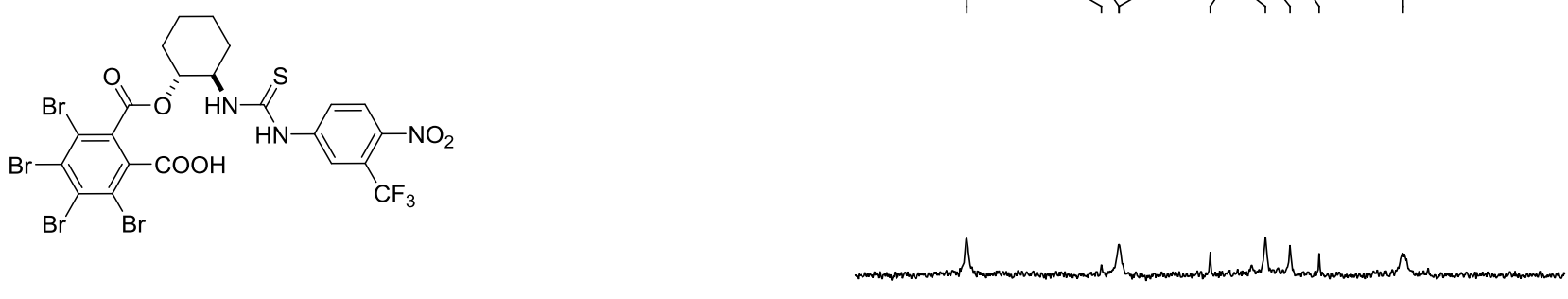

$\begin{array}{lllllllllll}128 & 127 & 126 & 125 & 124 & 123 & 122 & 121 & 120 & 119 & 118\end{array}$

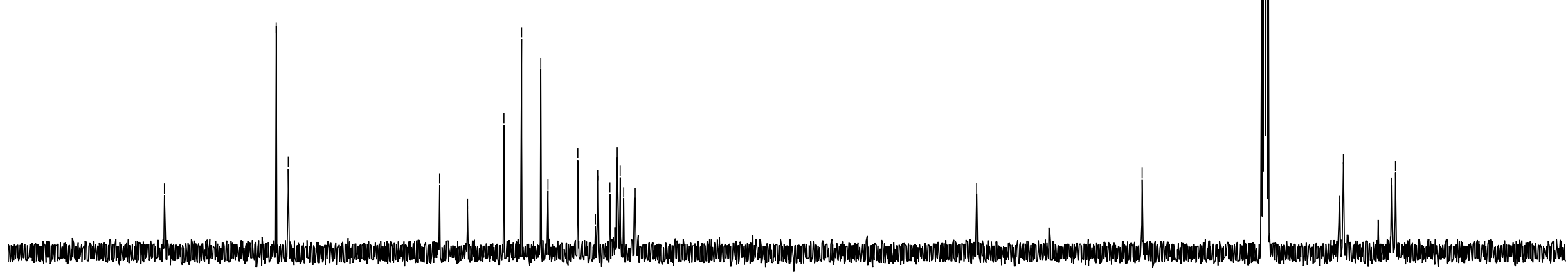


${ }^{19} \mathrm{~F}-\mathrm{NMR}\left(565 \mathrm{MHz},\left(\mathrm{CD}_{3}\right)_{2} \mathrm{SO}\right)$ of $\mathbf{4 f}$
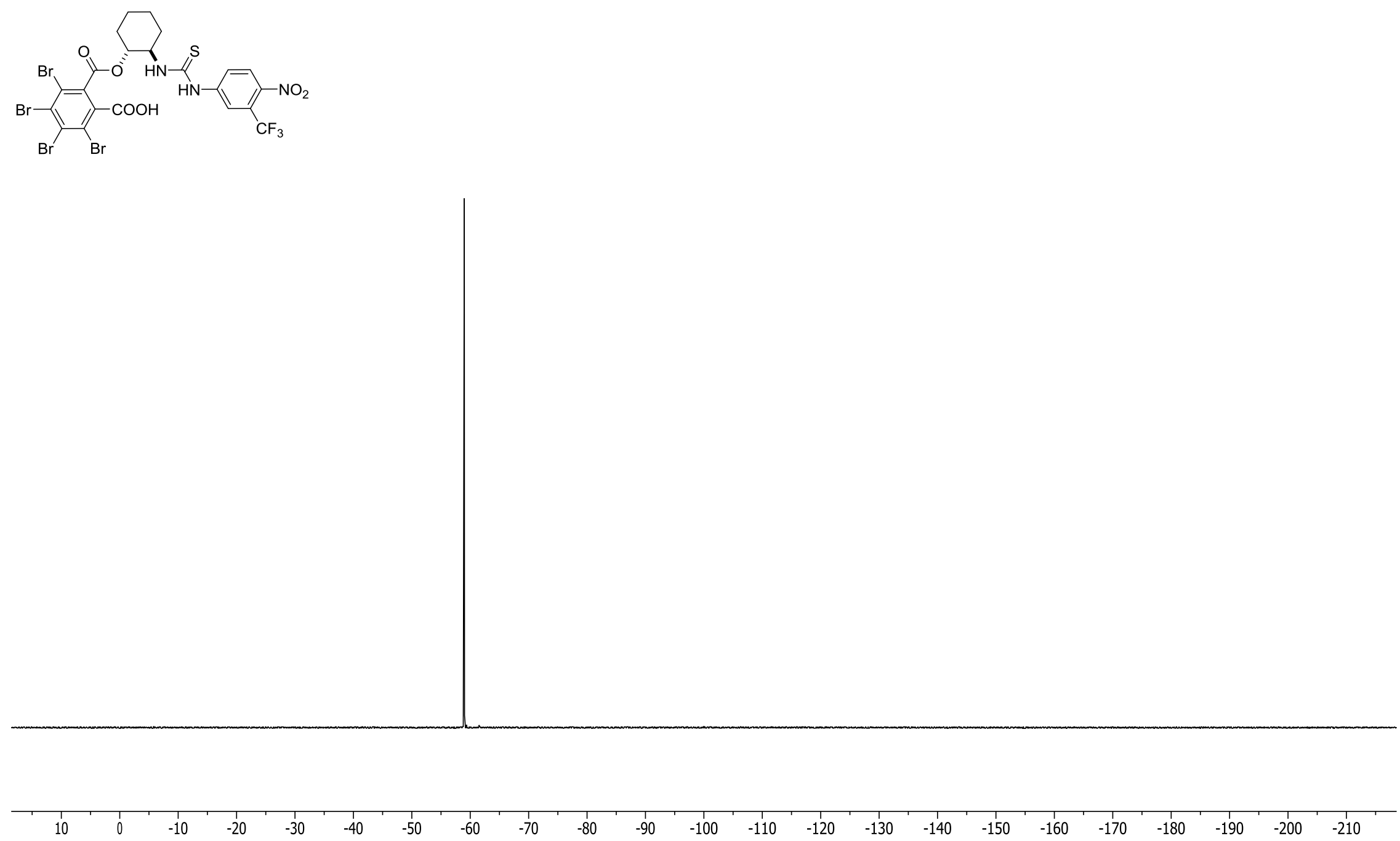
${ }^{1} \mathrm{H}-\mathrm{NMR}\left(600 \mathrm{MHz},\left(\mathrm{CD}_{3}\right)_{2} \mathrm{SO}\right)$ of $\mathbf{4 g}$
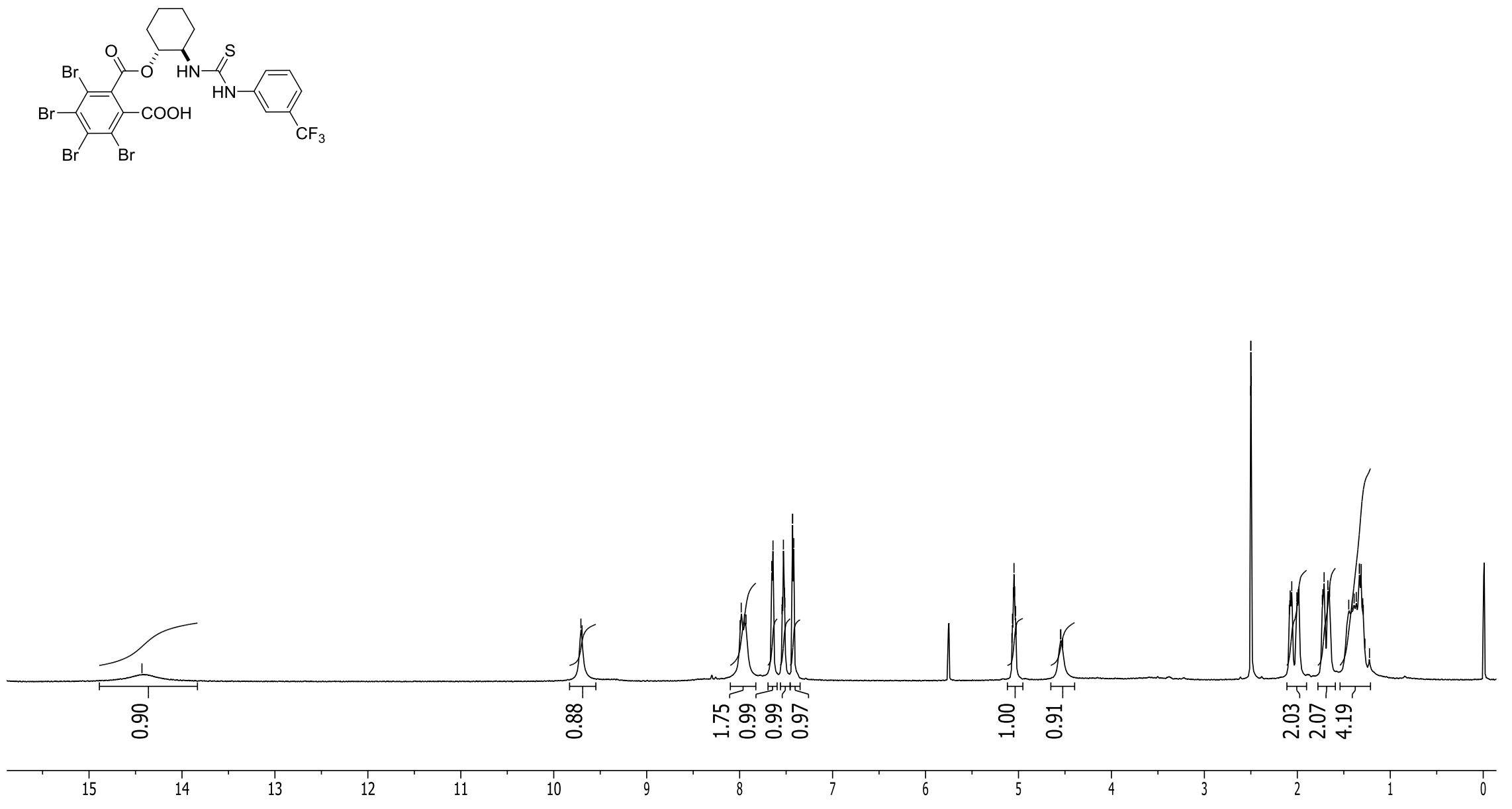


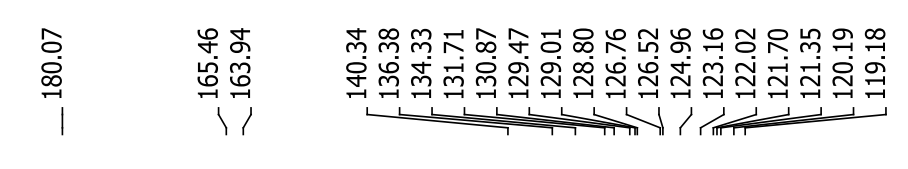

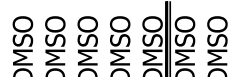

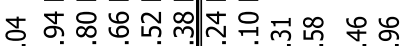

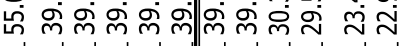
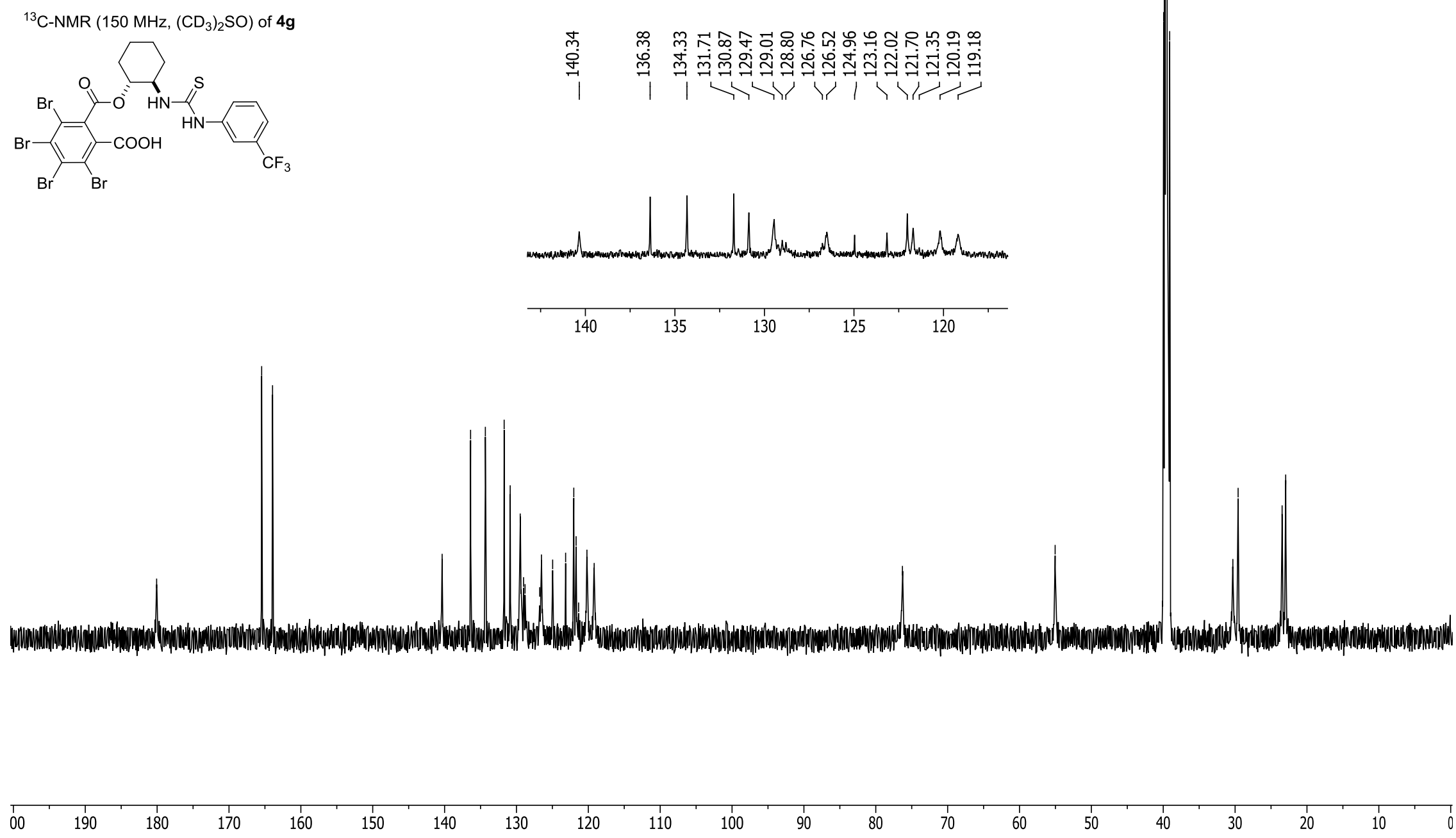
${ }^{19} \mathrm{~F}-\mathrm{NMR}\left(565 \mathrm{MHz},\left(\mathrm{CD}_{3}\right)_{2} \mathrm{SO}\right)$ of $\mathbf{4 g}$
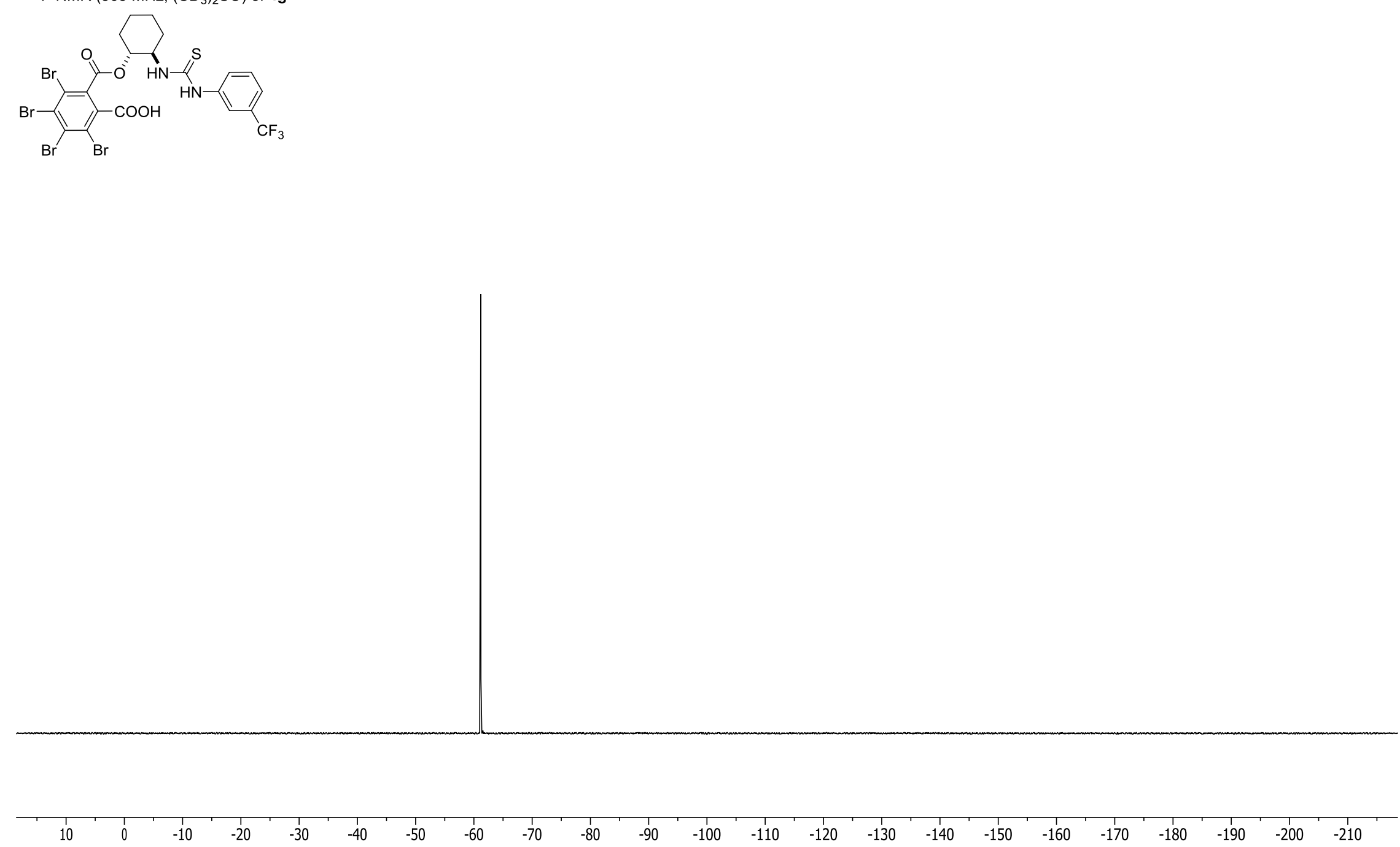
${ }^{1} \mathrm{H}-\mathrm{NMR}\left(600 \mathrm{MHz},\left(\mathrm{CD}_{3}\right)_{2} \mathrm{SO}\right)$ of $\mathbf{4 h}$
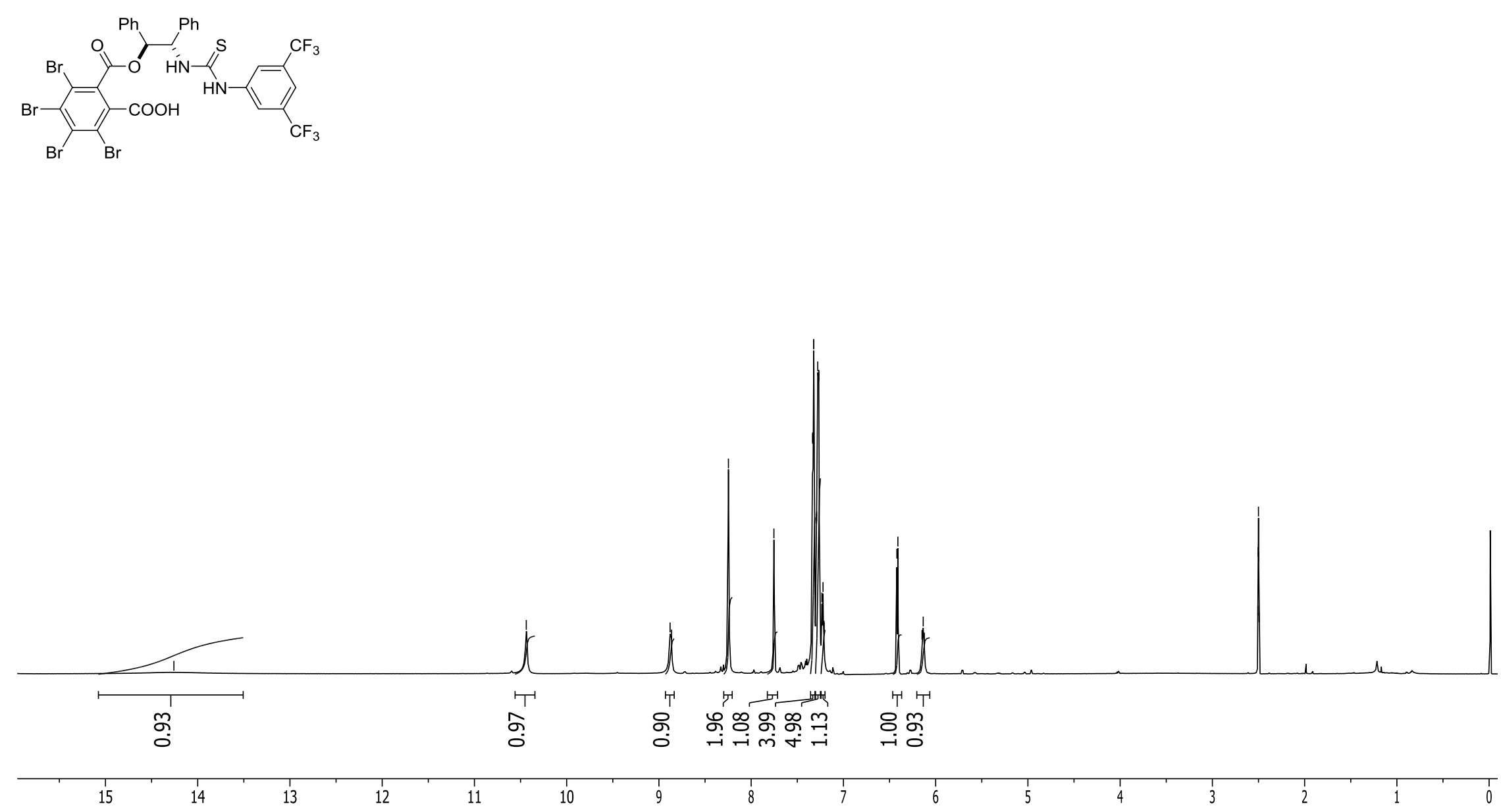


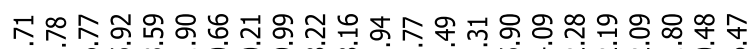

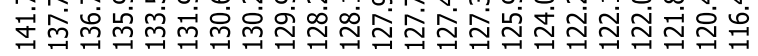
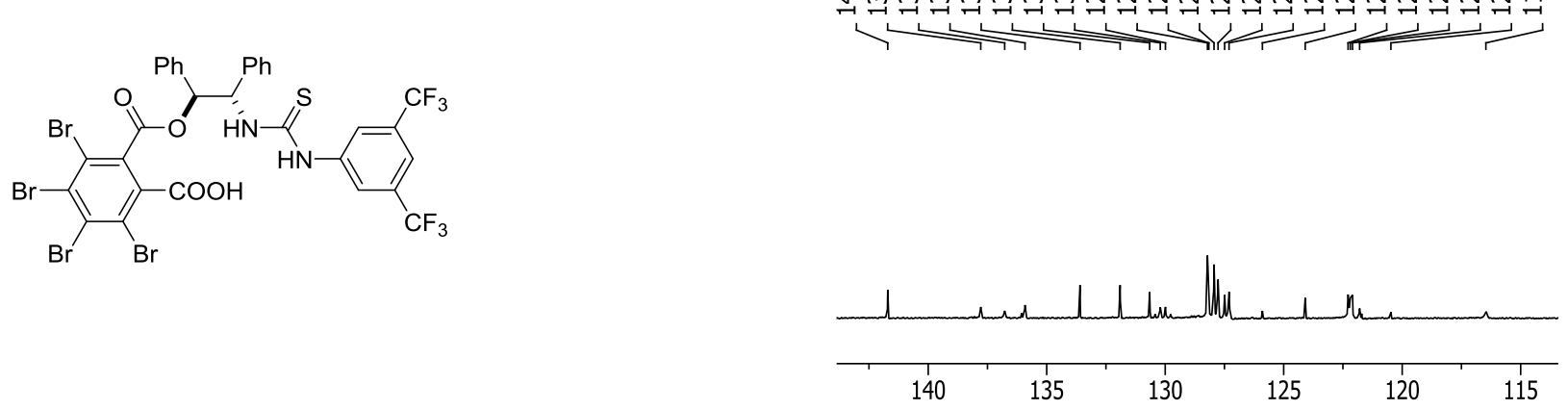

130
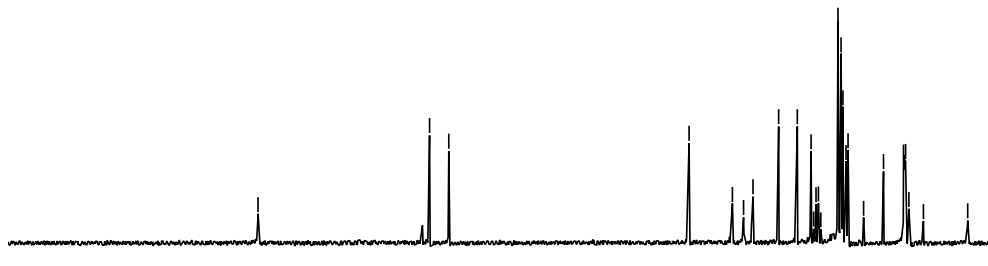

200

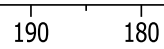

170

160

150

140

130

120

$110 \quad 100$
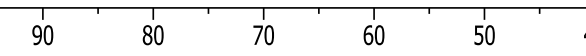
${ }^{19} \mathrm{~F}-\mathrm{NMR}\left(565 \mathrm{MHz},\left(\mathrm{CD}_{3}\right)_{2} \mathrm{SO}\right)$ of $4 \mathrm{~h}$
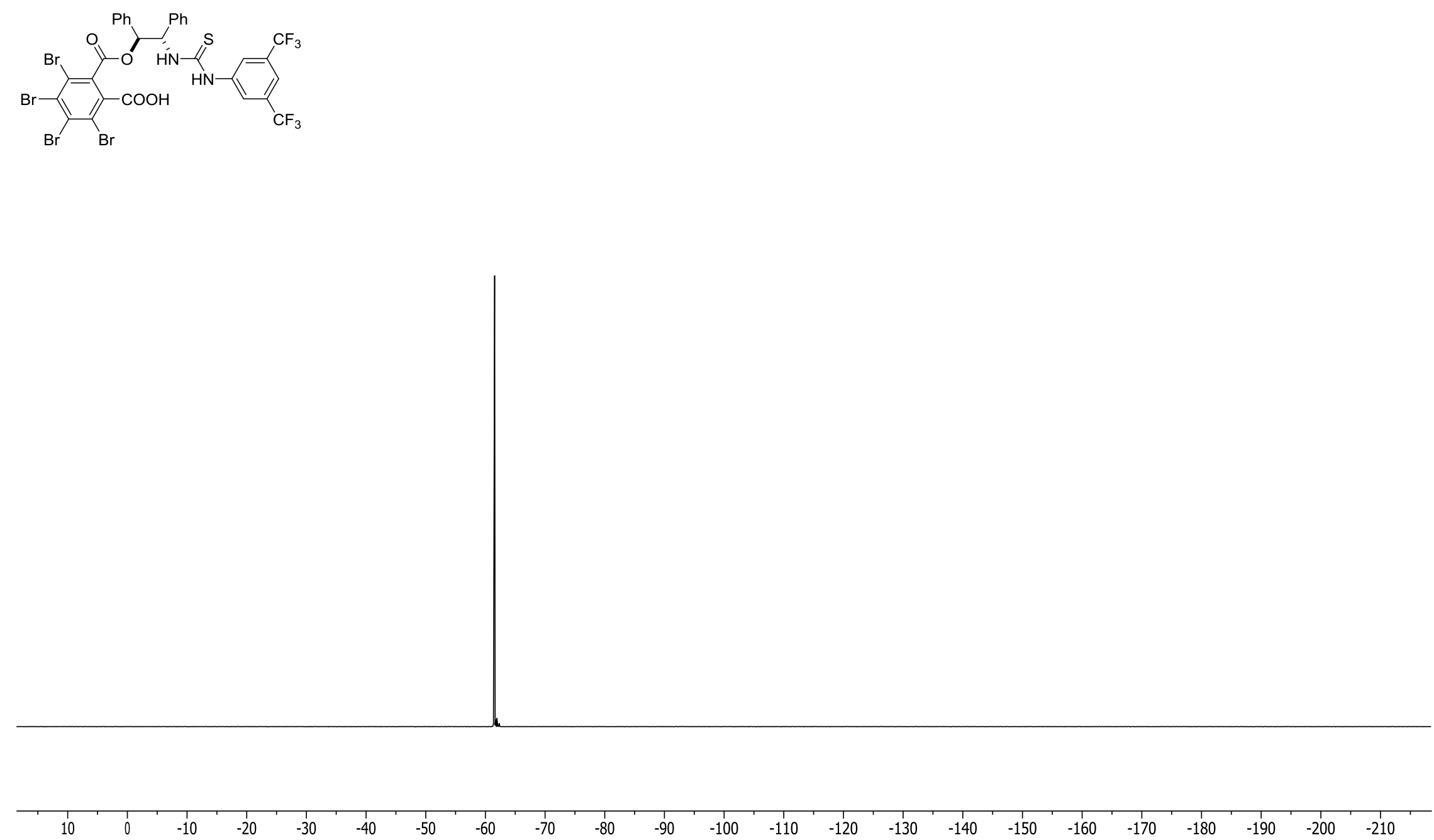
${ }^{1} \mathrm{H}-\mathrm{NMR}\left(600 \mathrm{MHz},\left(\mathrm{CD}_{3}\right)_{2} \mathrm{SO}\right)$ of $4 \mathbf{i}$
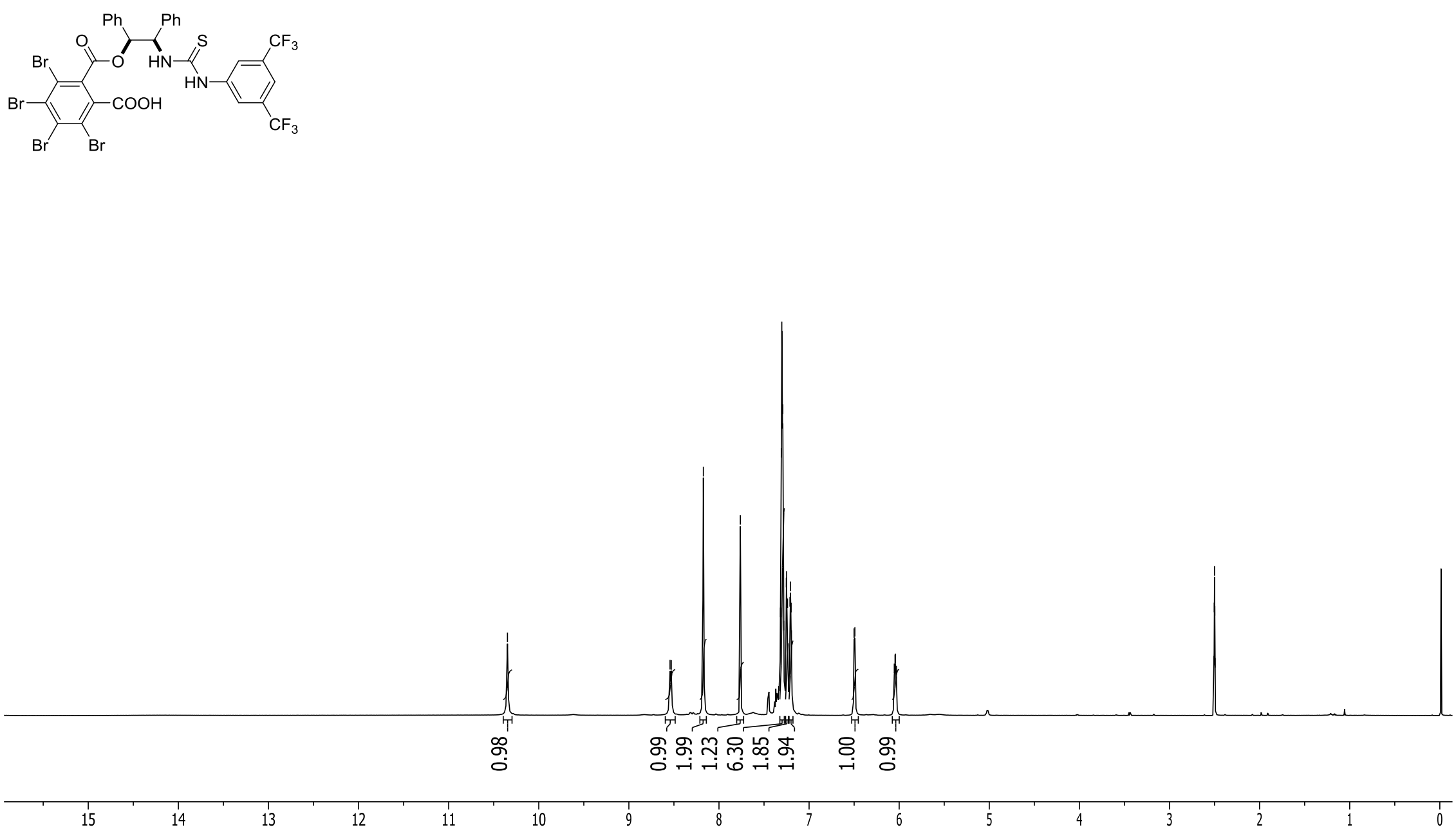
${ }^{13} \mathrm{C}-\mathrm{NMR}\left(150 \mathrm{MHz},\left(\mathrm{CD}_{3}\right)_{2} \mathrm{SO}\right)$ of $4 \mathbf{i}$

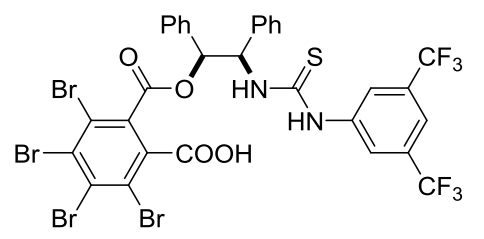

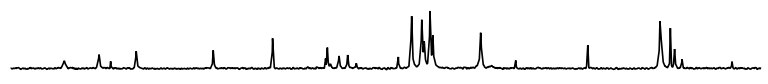
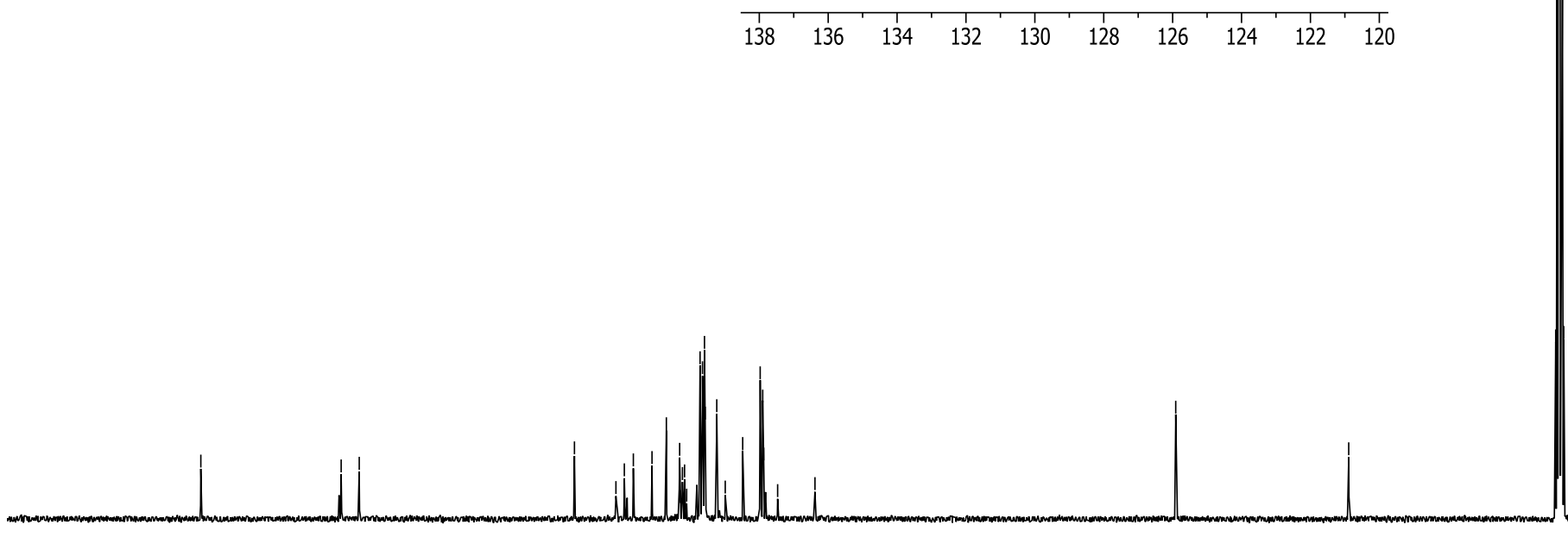

$30+190-180$

160

150

$140 \quad 130$

$120 \quad 110$

100

90

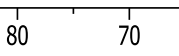

$60 \quad 50$ 
${ }^{19} \mathrm{~F}-\mathrm{NMR}\left(565 \mathrm{MHz},\left(\mathrm{CD}_{3}\right)_{2} \mathrm{SO}\right)$ of $4 \mathbf{i}$
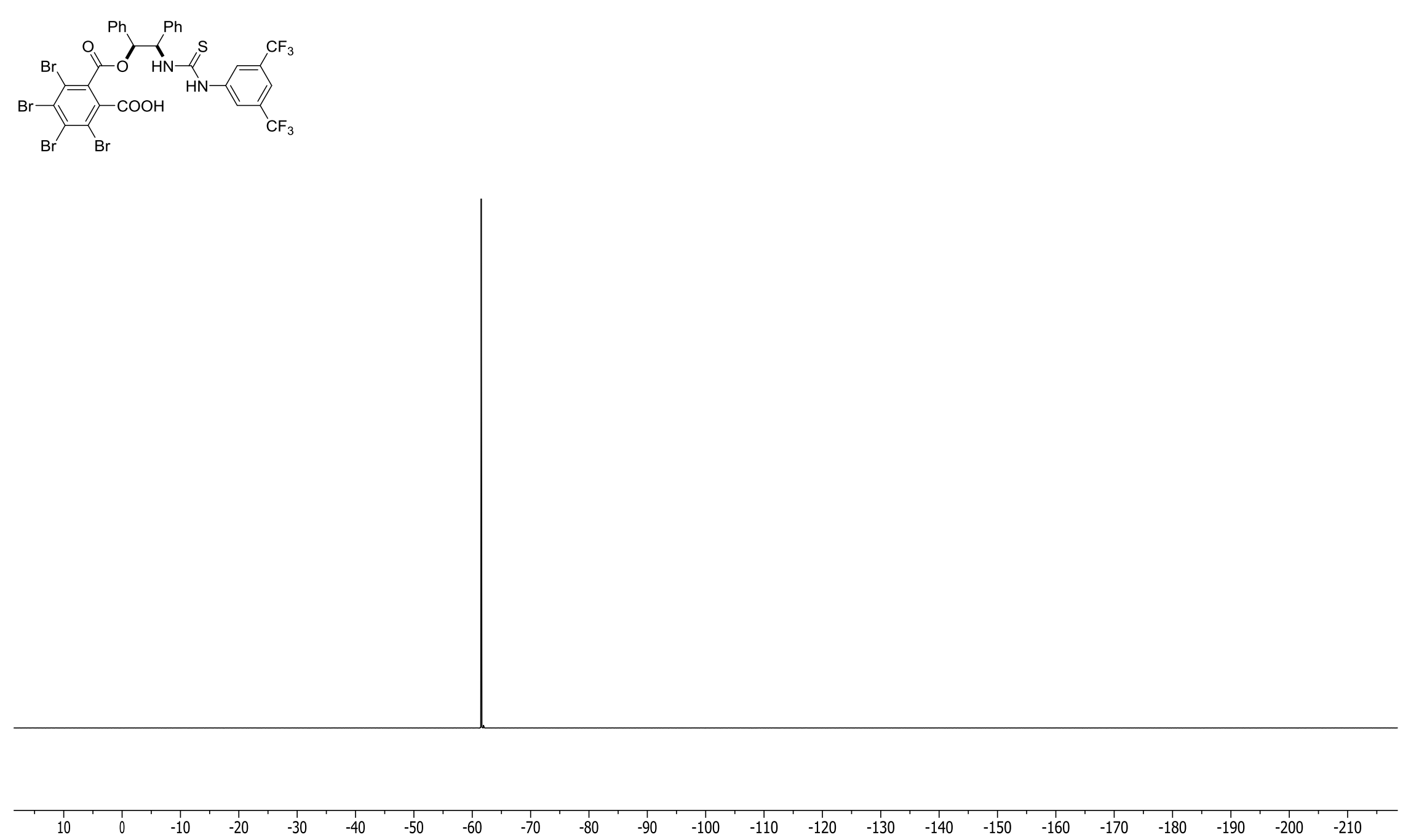


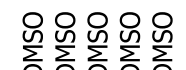

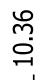

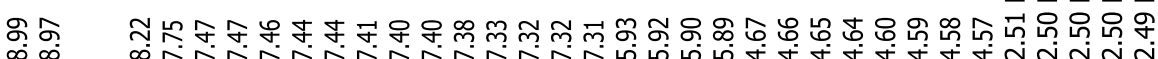

${ }^{1} \mathrm{H}-\mathrm{NMR}\left(600 \mathrm{MHz},\left(\mathrm{CD}_{3}\right)_{2} \mathrm{SO}\right)$ of $4 \mathrm{j}$
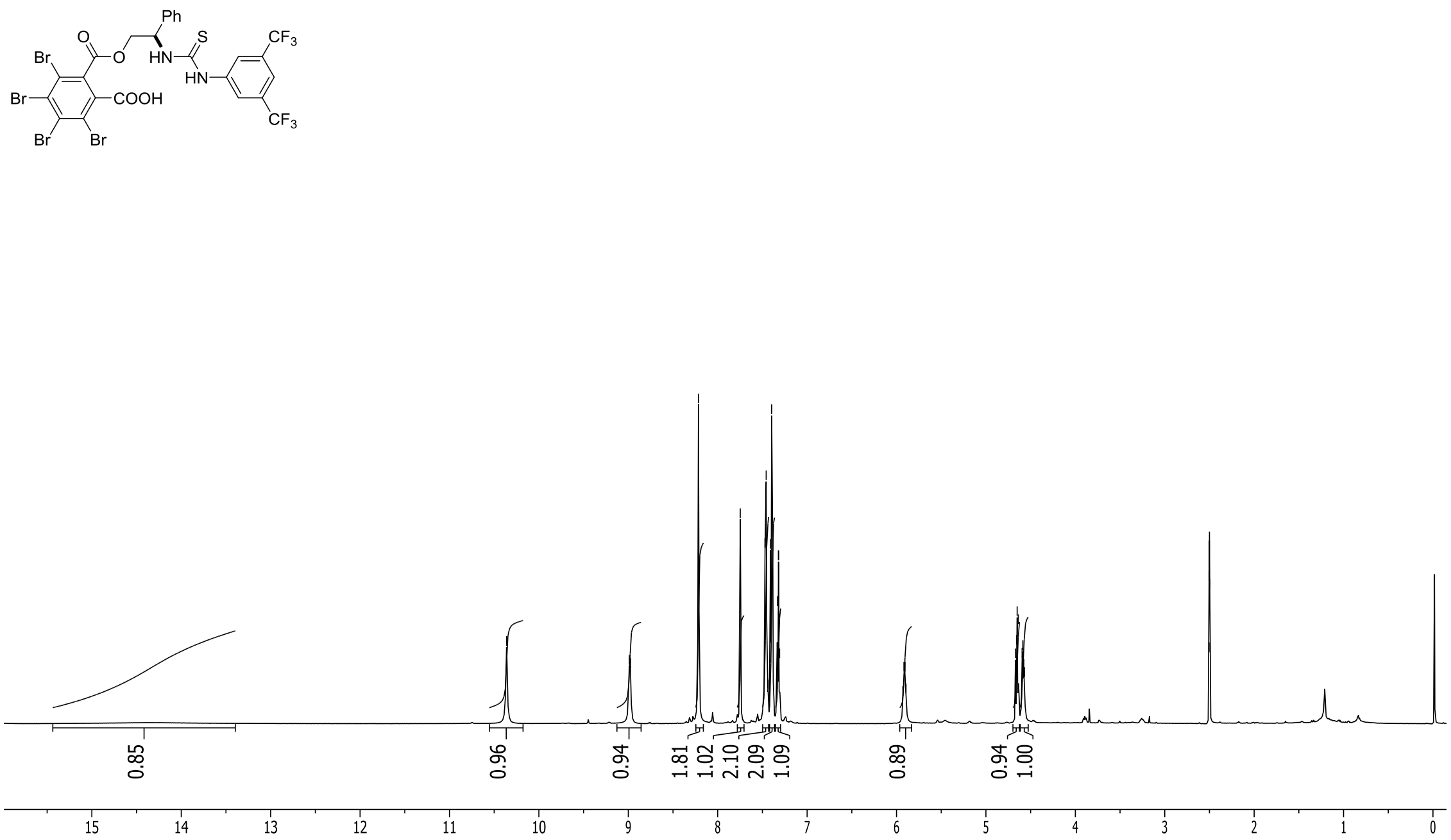


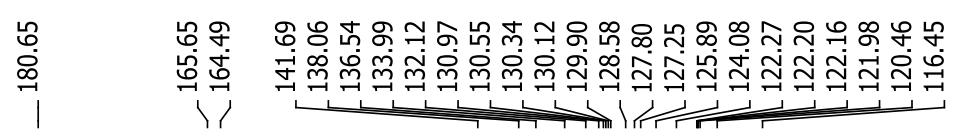

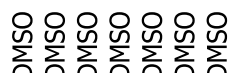

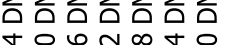

范

${ }^{13} \mathrm{C}-\mathrm{NMR}\left(150 \mathrm{MHz},\left(\mathrm{CD}_{3}\right)_{2} \mathrm{SO}\right)$ of $4 \mathrm{j}$

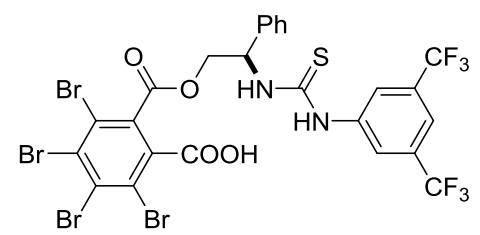

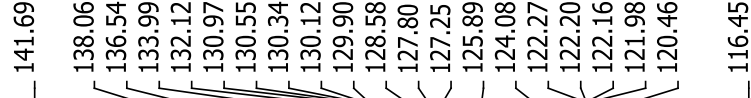
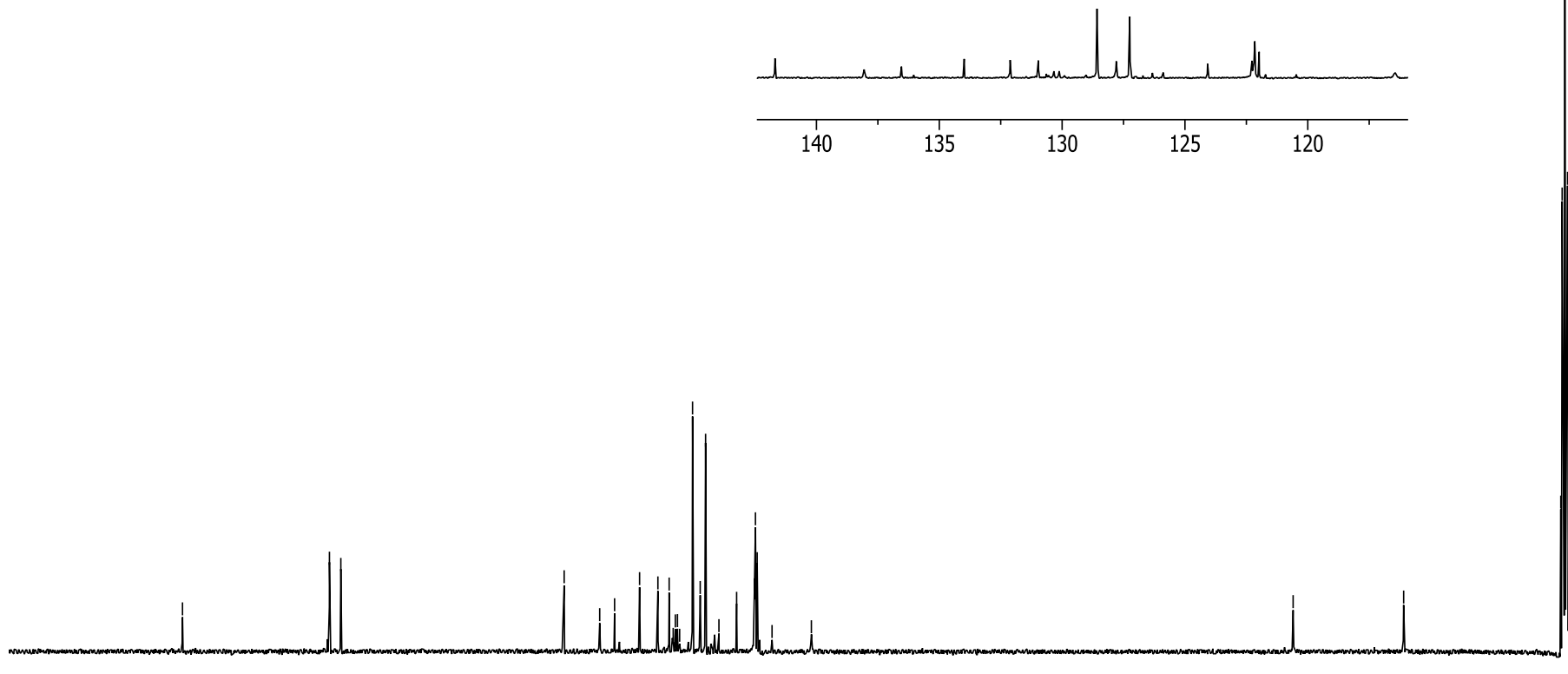

$190 \quad 180$

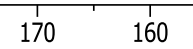

150

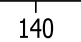

130

$120 \quad 110$ 
${ }^{19} \mathrm{~F}-\mathrm{NMR}\left(565 \mathrm{MHz},\left(\mathrm{CD}_{3}\right)_{2} \mathrm{SO}\right)$ of $4 \mathrm{j}$
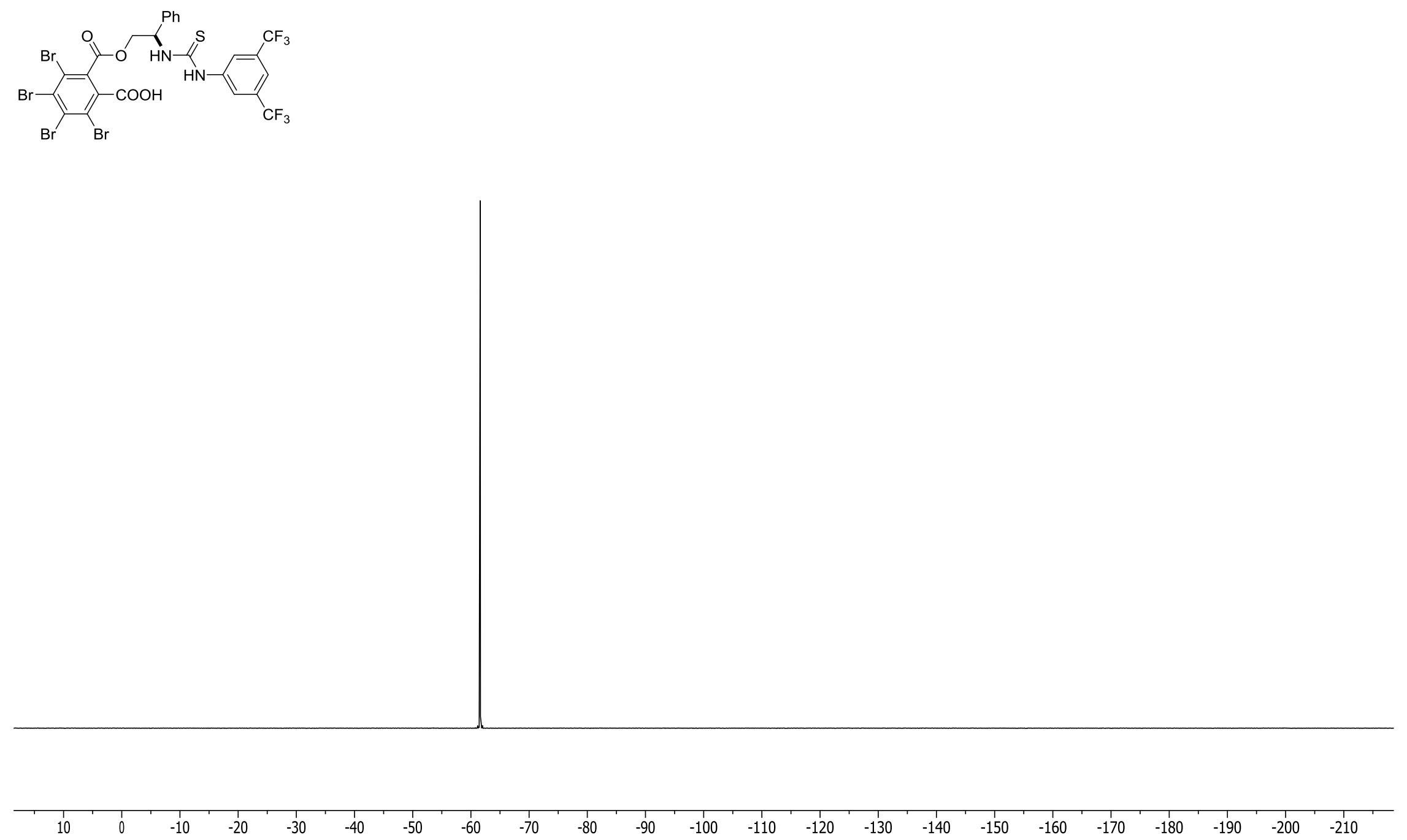
${ }^{1} \mathrm{H}-\mathrm{NMR}\left(600 \mathrm{MHz},\left(\mathrm{CD}_{3}\right)_{2} \mathrm{SO}\right)$ of $\mathbf{4 k}$

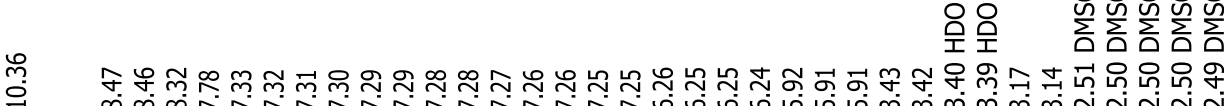
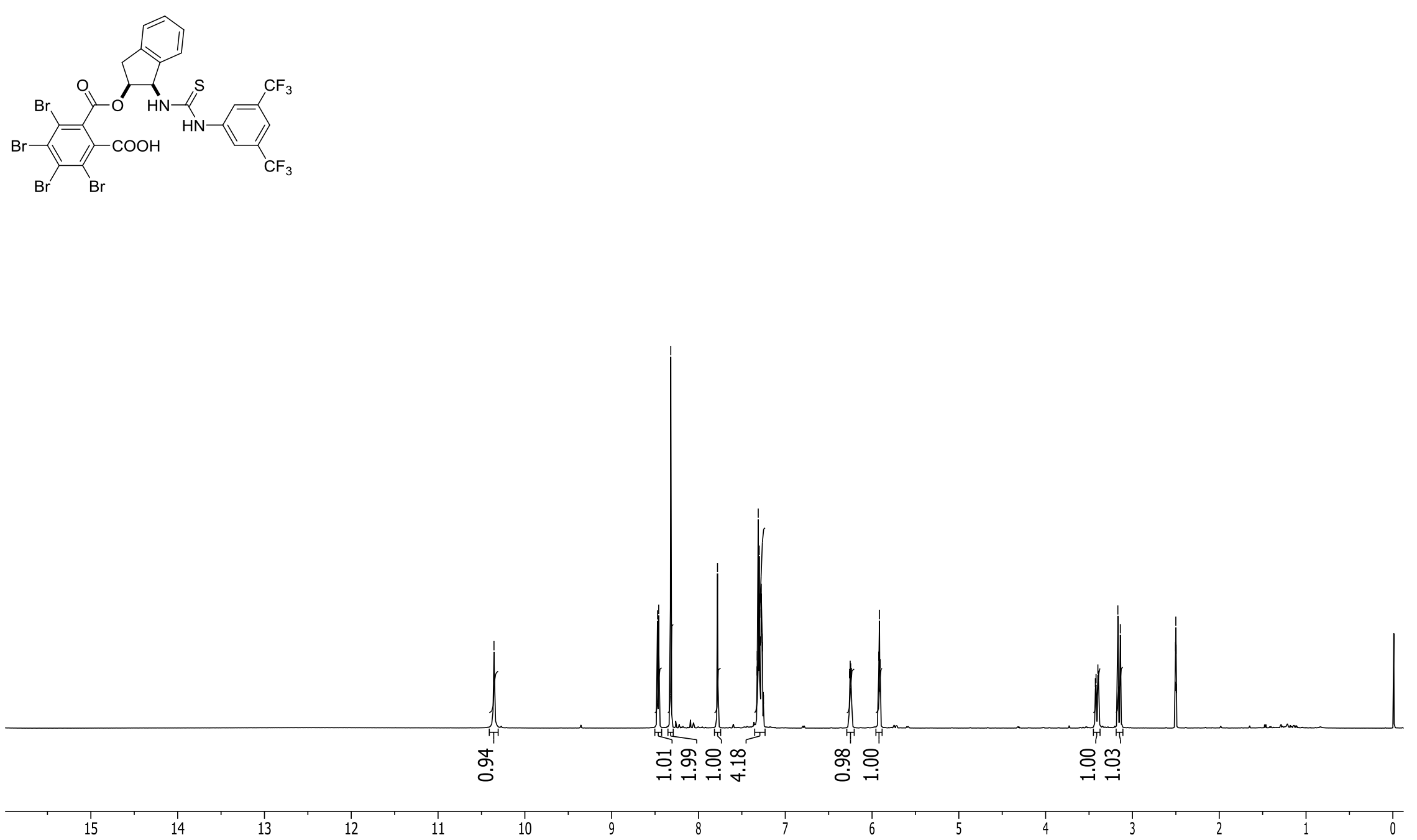

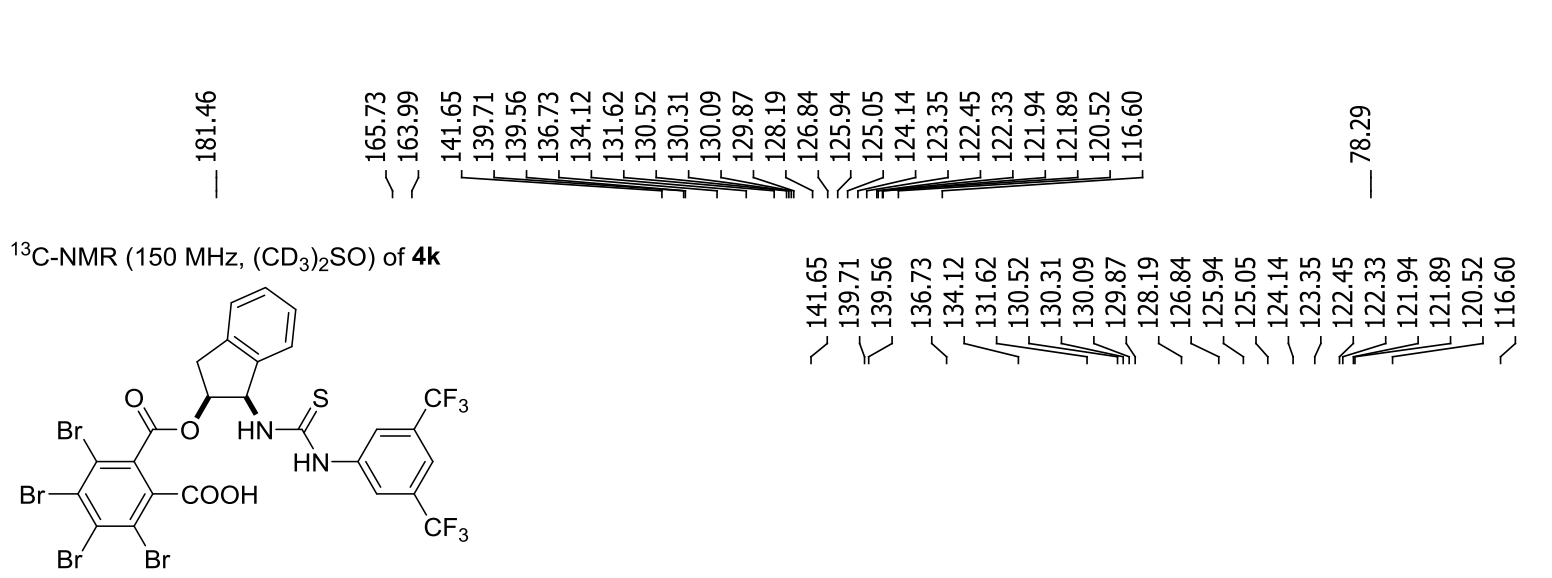

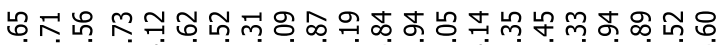

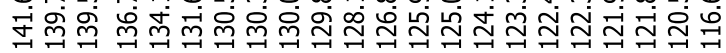
।
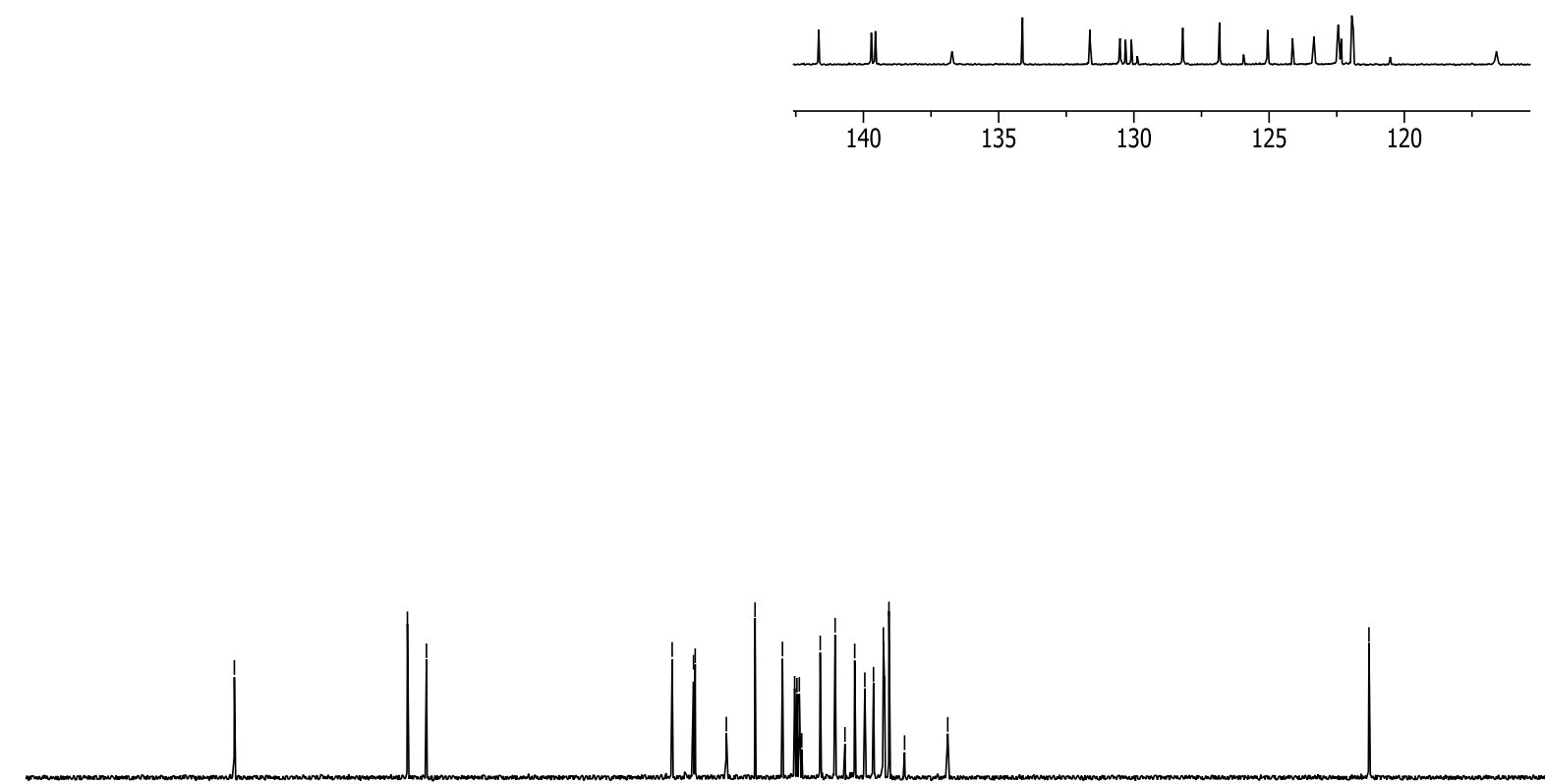

זี่

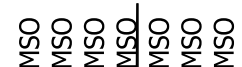

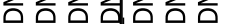

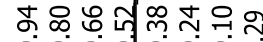

लें

100

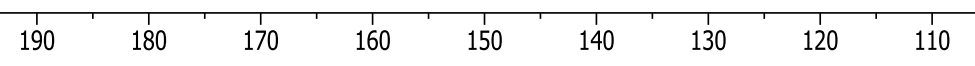

100

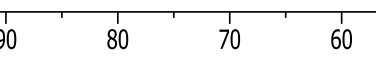

50 
${ }^{19} \mathrm{~F}-\mathrm{NMR}\left(565 \mathrm{MHz},\left(\mathrm{CD}_{3}\right)_{2} \mathrm{SO}\right)$ of $\mathbf{4 k}$
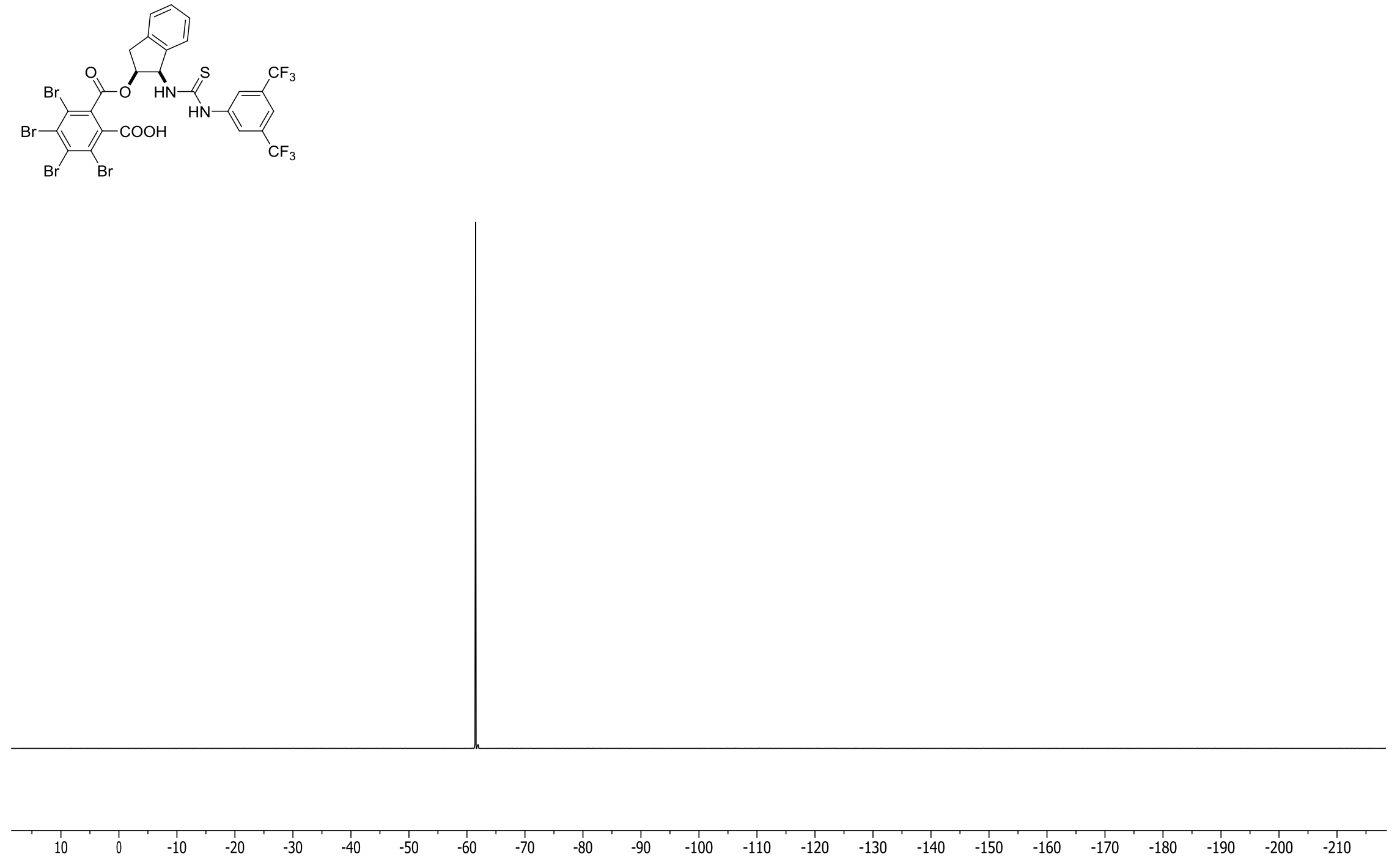
ర్రิ

웅

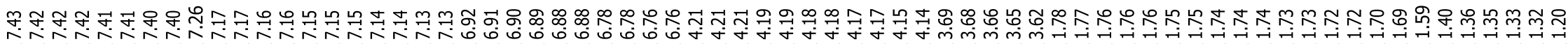
${ }^{1} \mathrm{H}-\mathrm{NMR}\left(400 \mathrm{MHz}, \mathrm{CDCl}_{3}\right)$ of $3 \mathbf{a}$
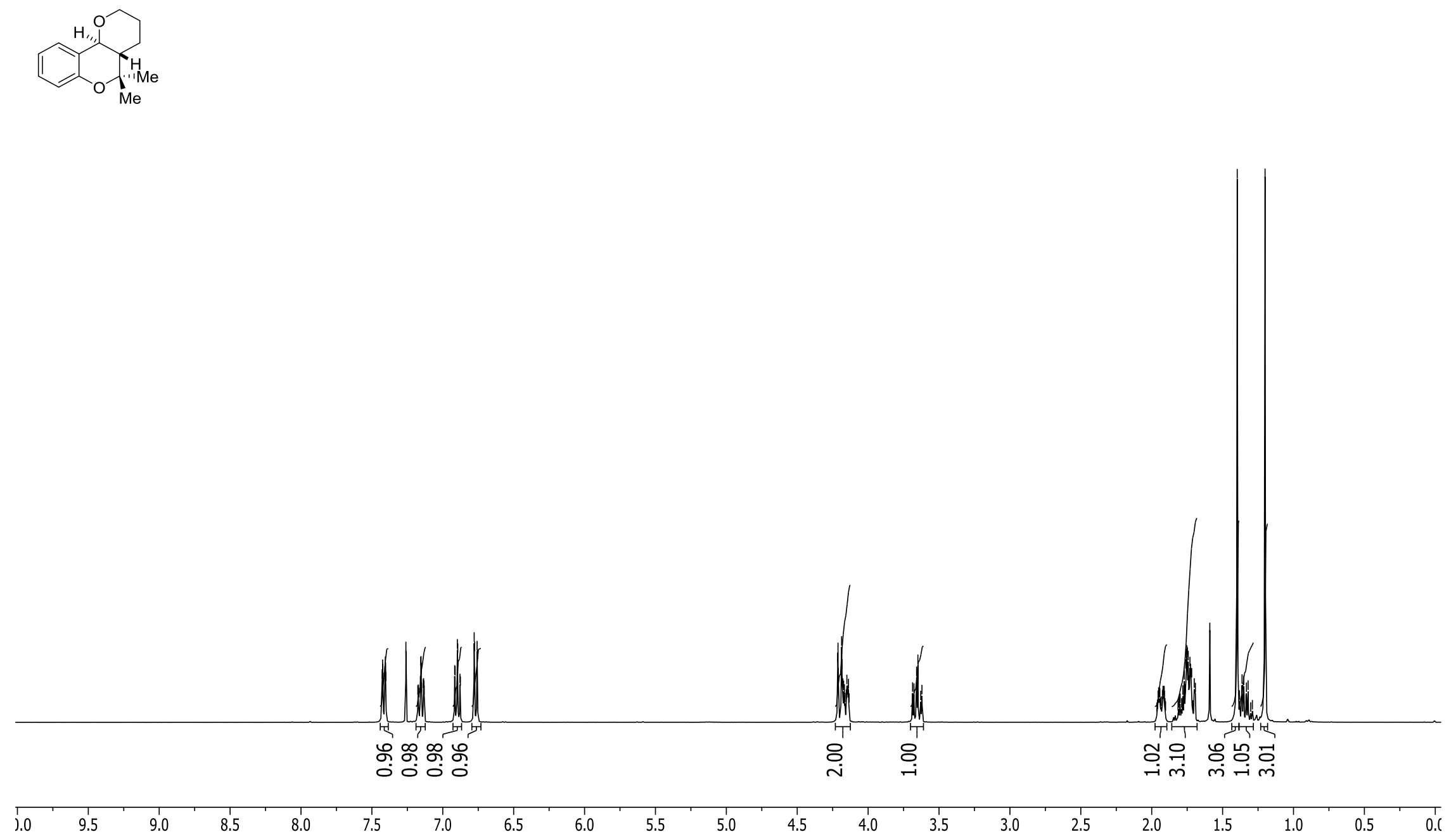


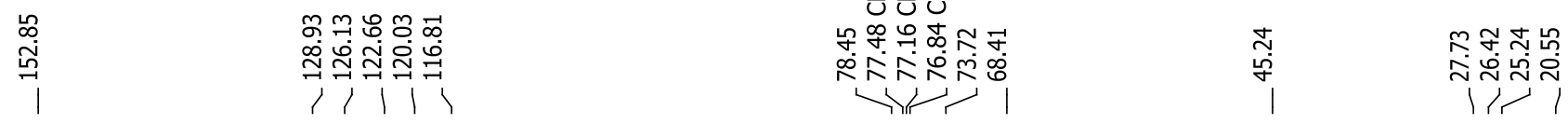

${ }^{13} \mathrm{C}-\mathrm{NMR}\left(100 \mathrm{MHz}, \mathrm{CDCl}_{3}\right)$ of $\mathbf{3 a}$<smiles>CC1(C)Oc2ccccc2[C@H]2OCCC[C@@H]21</smiles>
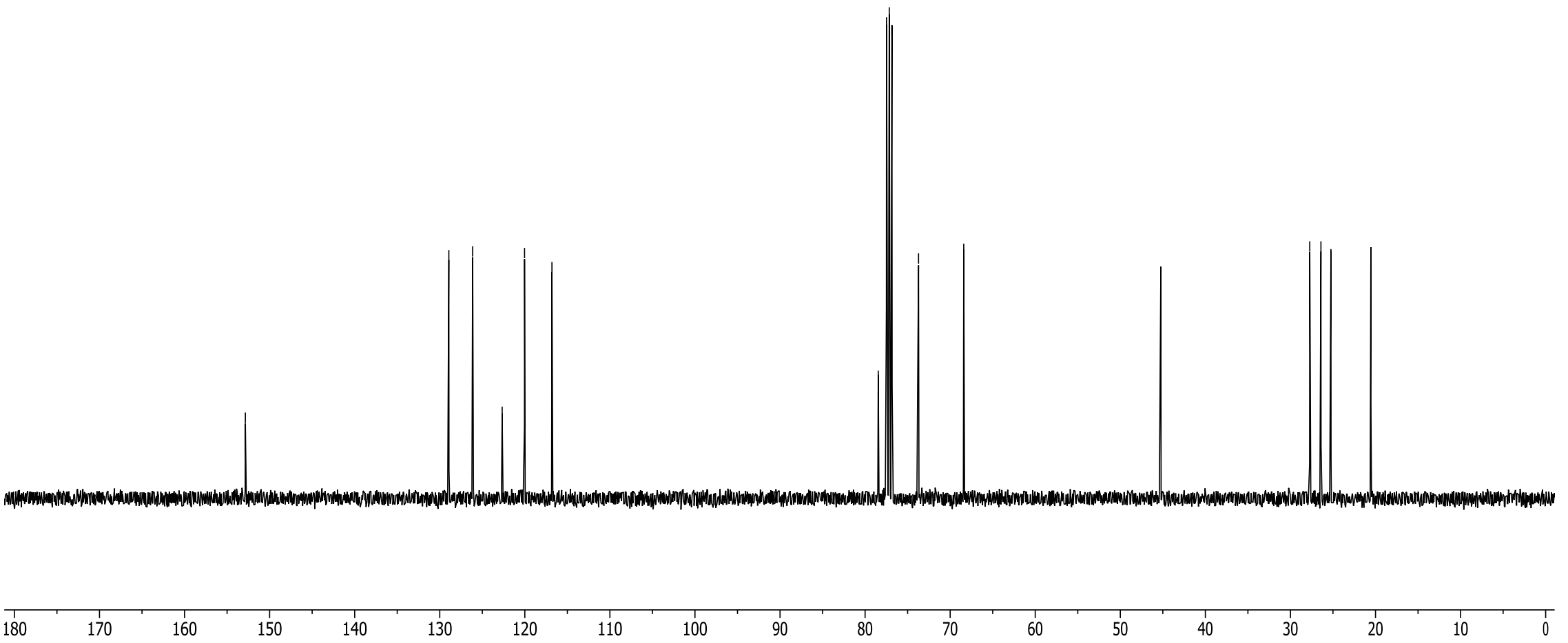


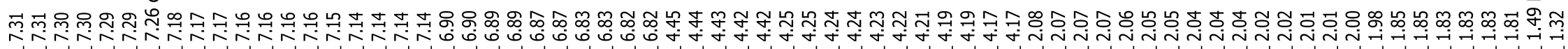
得

${ }^{1} \mathrm{H}-\mathrm{NMR}\left(500 \mathrm{MHz}, \mathrm{CDCl}_{3}\right)$ of $\mathbf{3 b}$
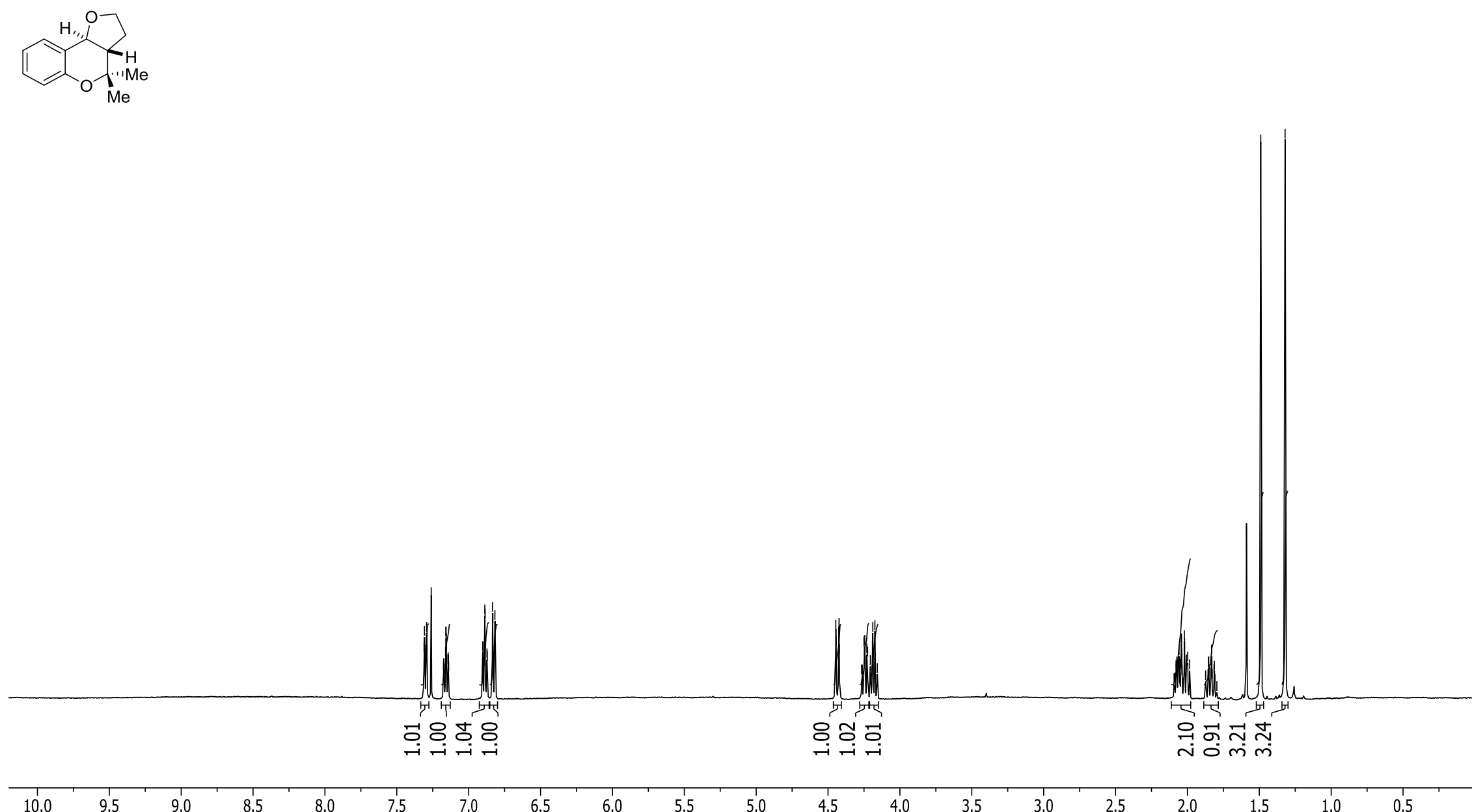
总

${ }^{13} \mathrm{C}-\mathrm{NMR}\left(125 \mathrm{MHz}, \mathrm{CDCl}_{3}\right.$ ) of $\mathbf{3 b}$

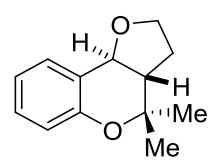

$\frac{m}{\bar{v}} \frac{m}{\bar{V}} \frac{m}{\mathrm{~V}}$

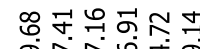

হNN采

놉

오옹

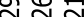

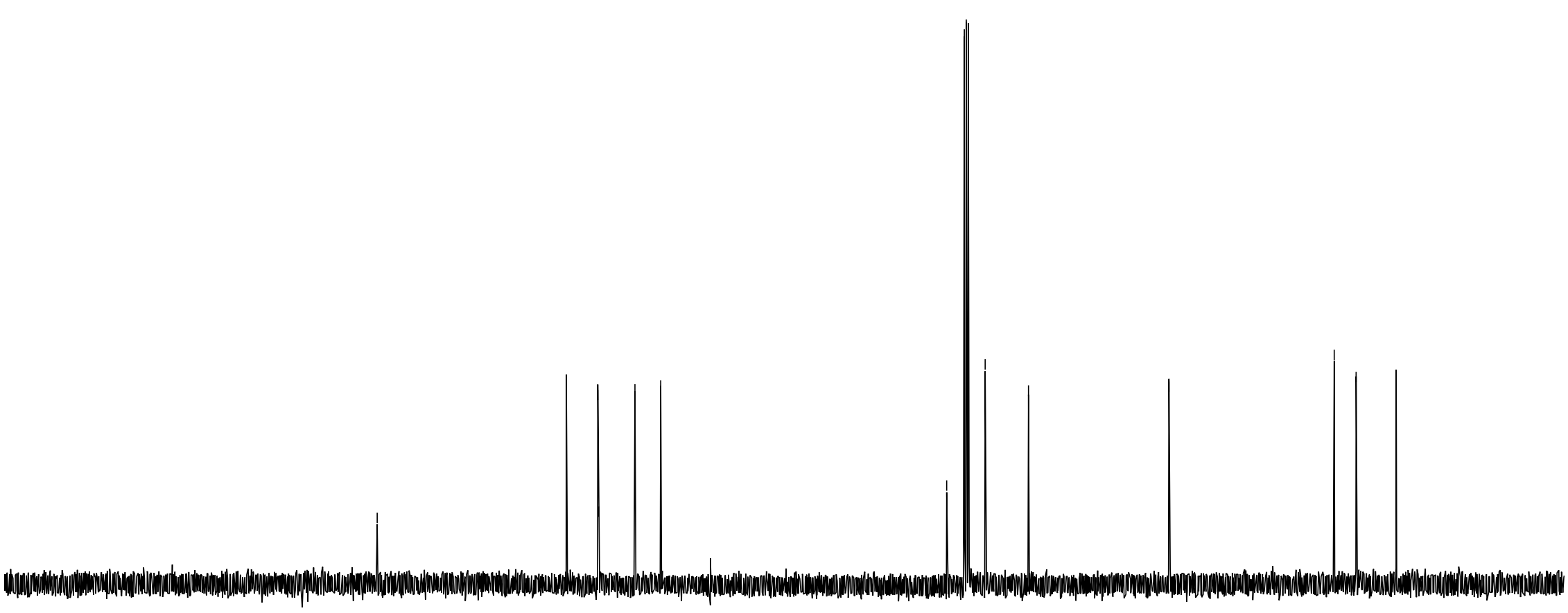

200 
응

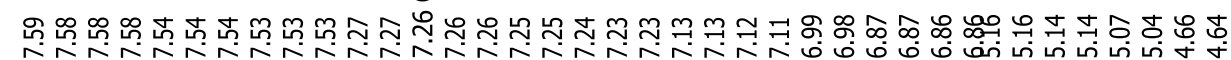
促

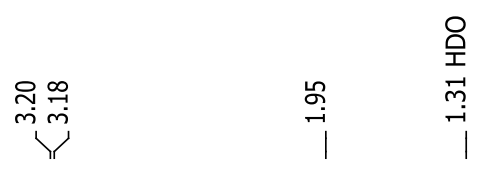

${ }^{1} \mathrm{H}-\mathrm{NMR}\left(600 \mathrm{MHz}, \mathrm{CDCl}_{3}\right)$ of $3 \mathrm{c}$
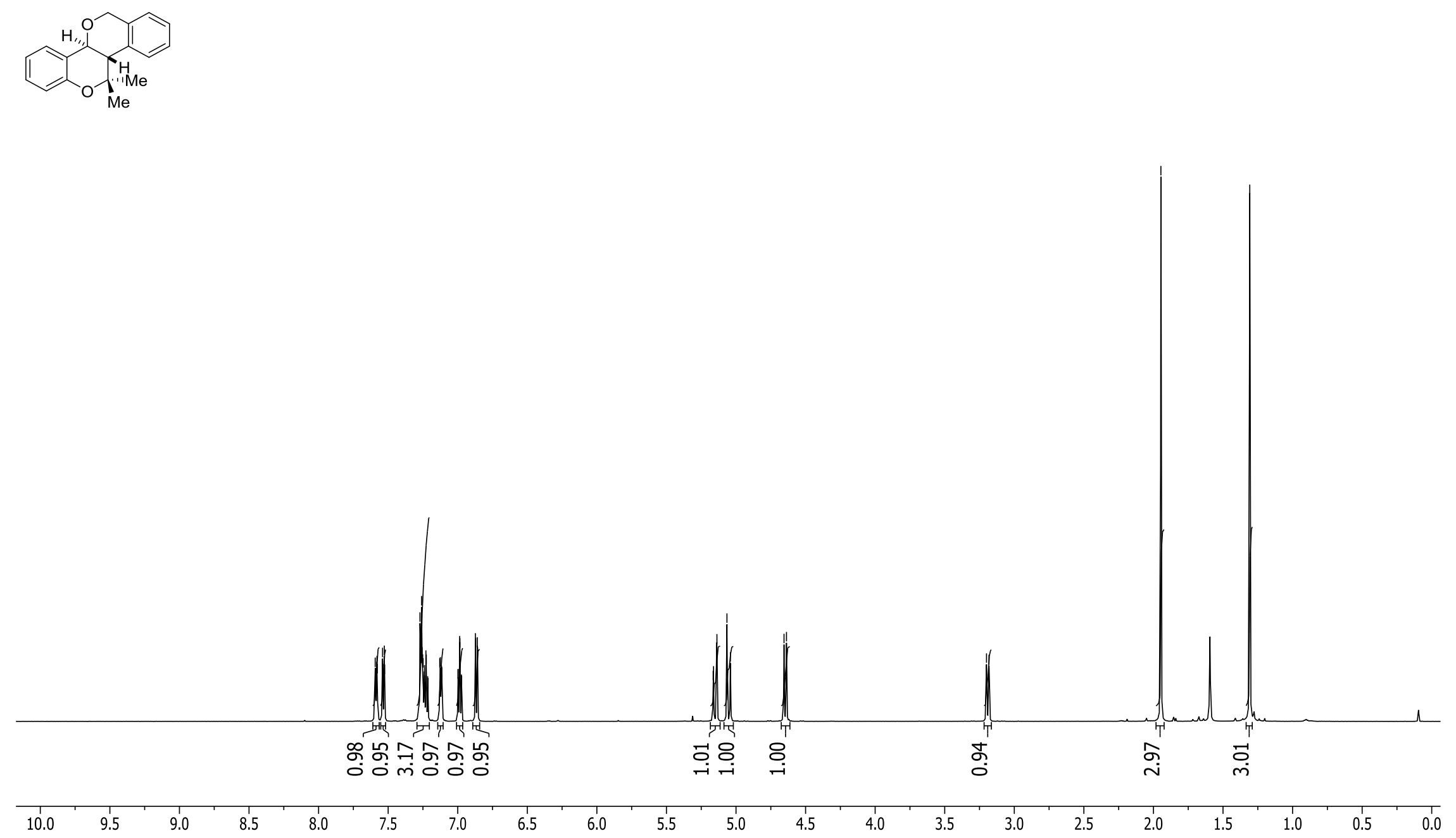


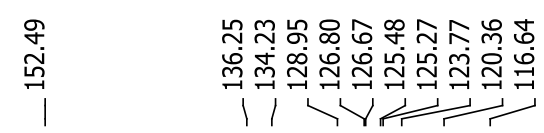

\section{융}

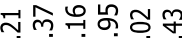

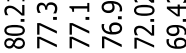

। ए।

${ }^{13} \mathrm{C}-\mathrm{NMR}\left(150 \mathrm{MHz}, \mathrm{CDCl}_{3}\right)$ of $3 \mathrm{c}$
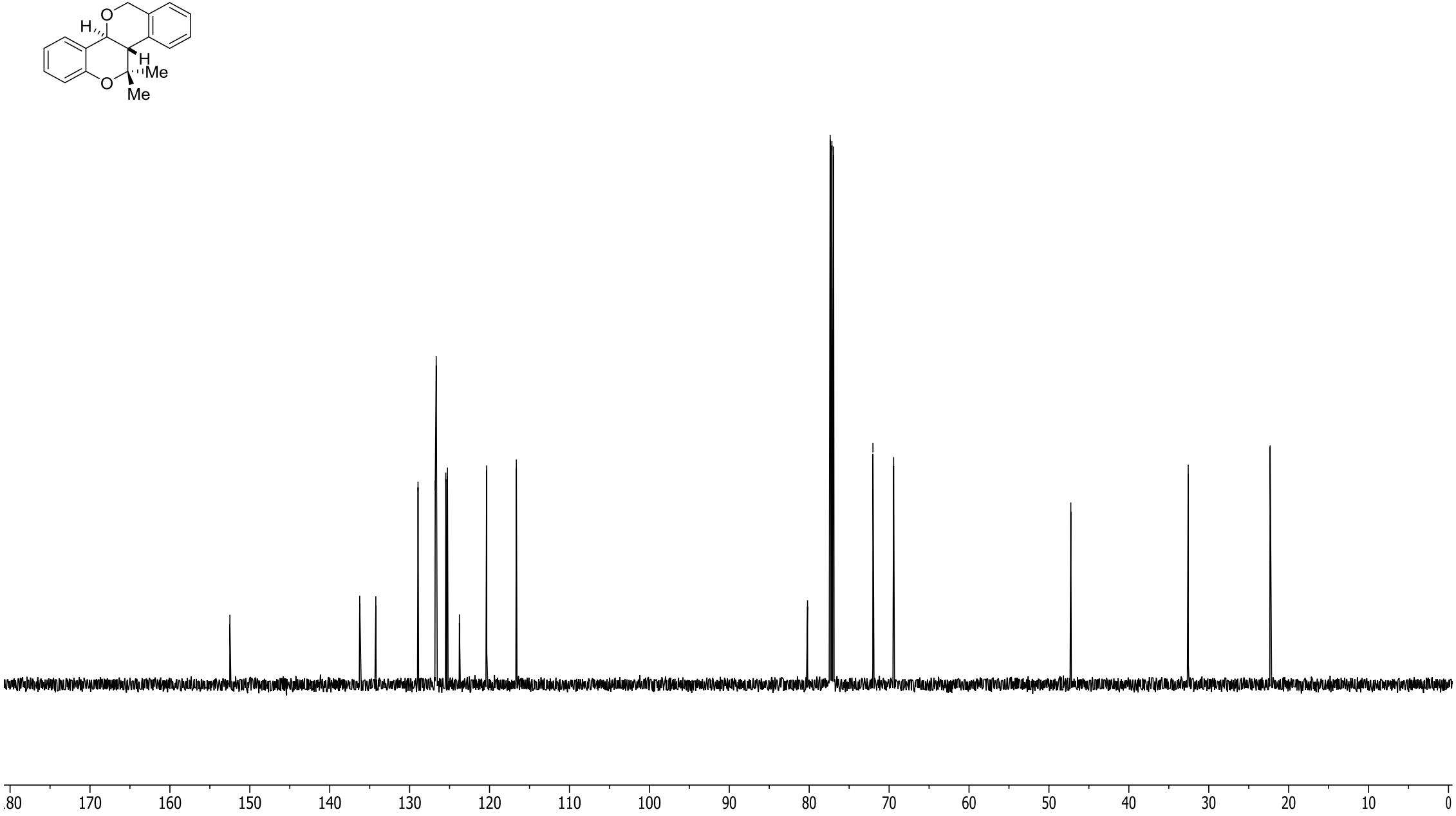

140

130

120

110

100

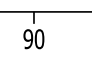

80

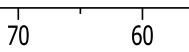

40

$20 \quad 10$ 


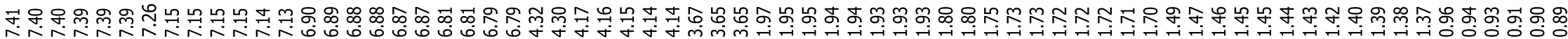
${ }^{1} \mathrm{H}-\mathrm{NMR}\left(600 \mathrm{MHz}, \mathrm{CDCl}_{3}\right)$ of $\mathbf{3 d}$
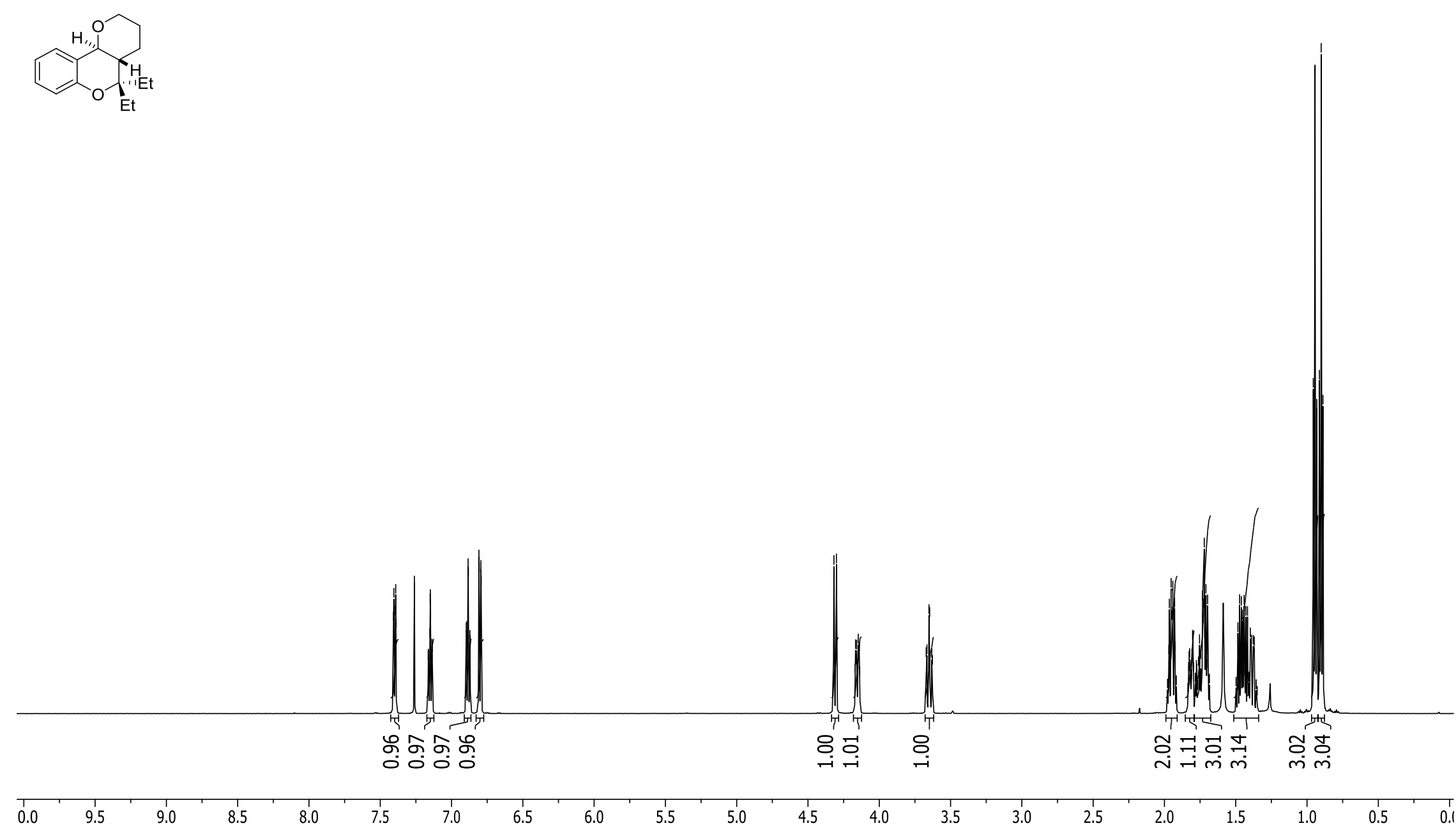

5.5 


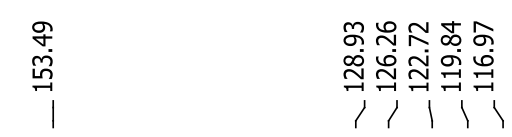

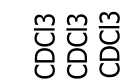

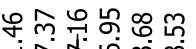

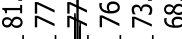

${ }^{13} \mathrm{C}-\mathrm{NMR}\left(150 \mathrm{MHz}, \mathrm{CDCl}_{3}\right)$ of $\mathbf{3 d}$

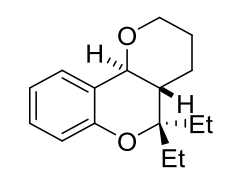




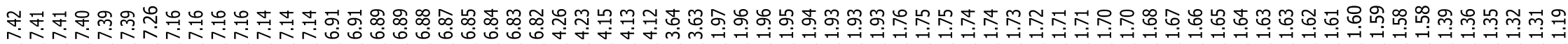

${ }^{1} \mathrm{H}-\mathrm{NMR}\left(400 \mathrm{MHz}, \mathrm{CDCl}_{3}\right)$ of $3 \mathbf{e}$

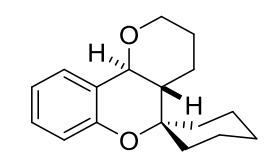

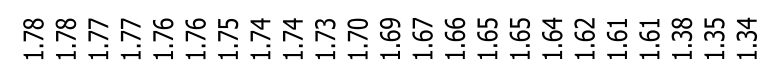

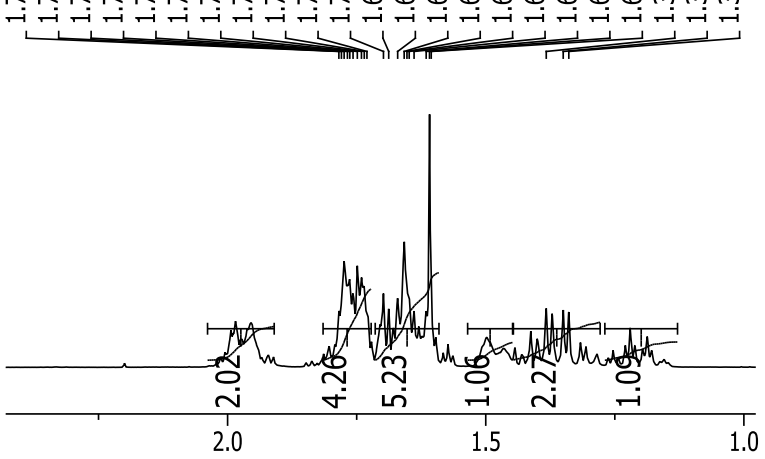

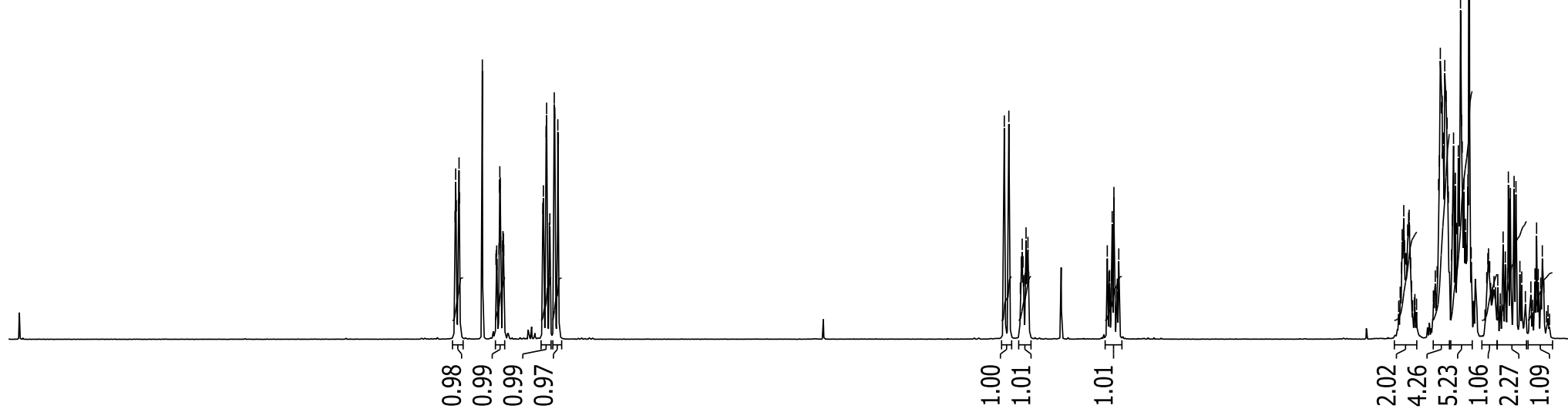
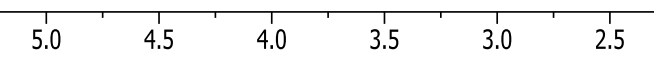

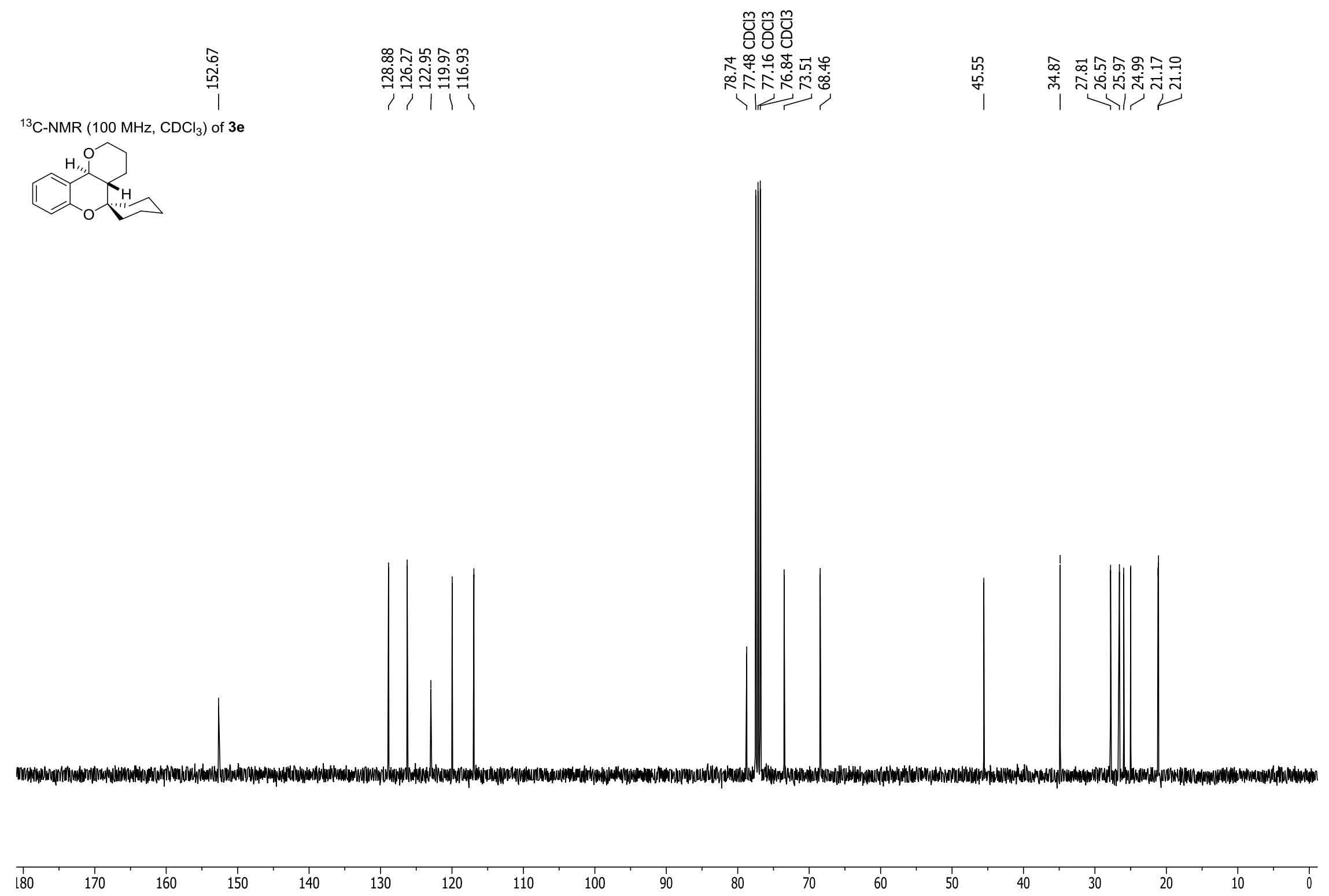
음

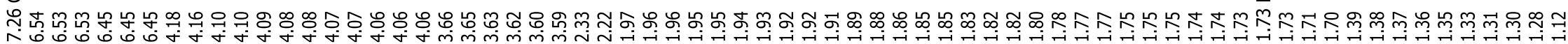

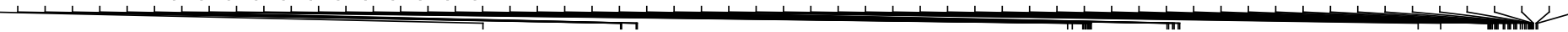

${ }^{1} \mathrm{H}-\mathrm{NMR}\left(400 \mathrm{MHz}, \mathrm{CDCl}_{3}\right)$ of $\mathbf{3 f}$
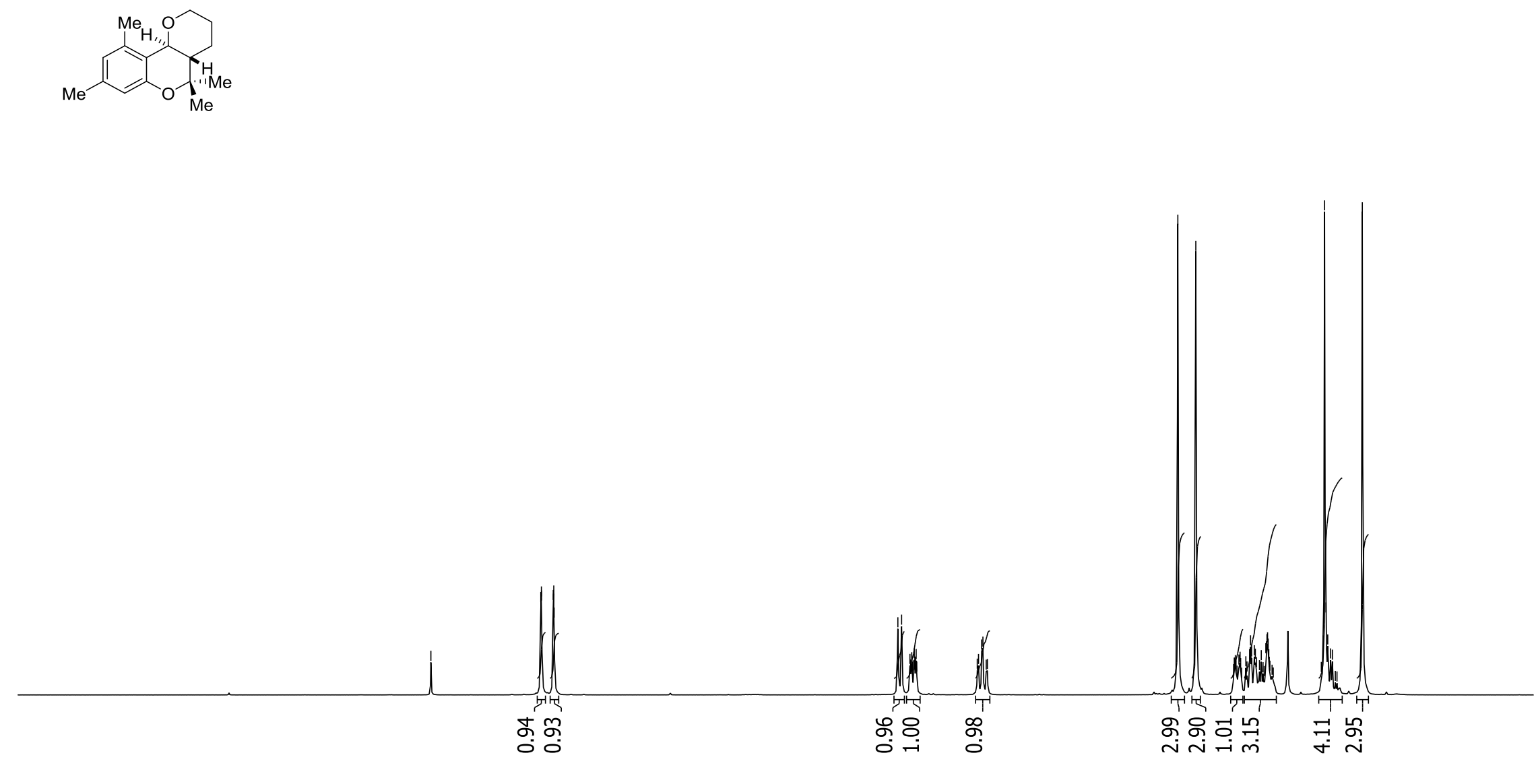

$9.5 \quad 9.0$

$6.5 \quad 6.0$
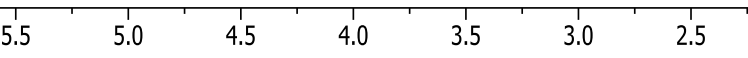


| v

商旁旁

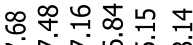

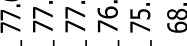

调

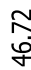

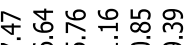

걱

${ }^{13} \mathrm{C}-\mathrm{NMR}\left(100 \mathrm{MHz}, \mathrm{CDCl}_{3}\right)$ of $\mathbf{3 f}$
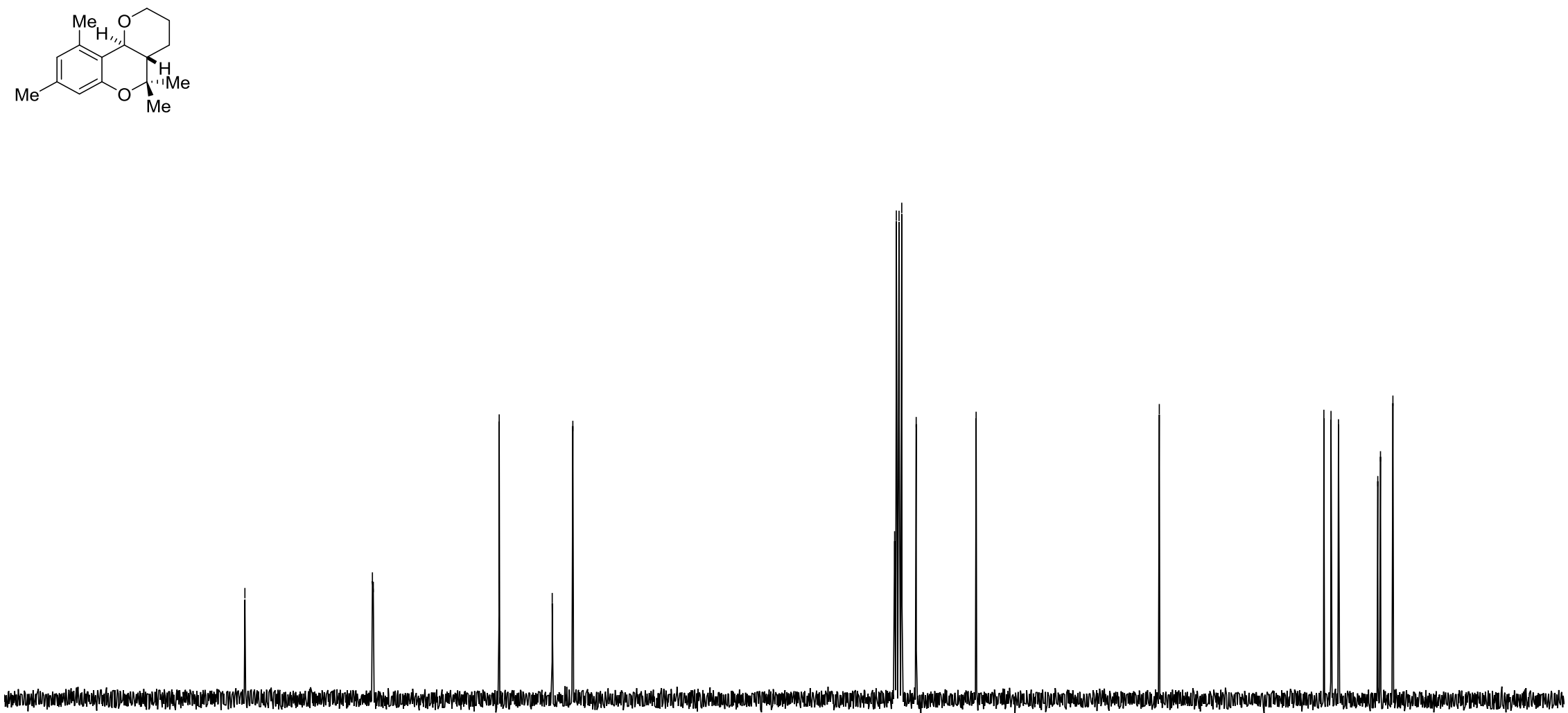

$\frac{1}{180}$

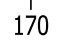

160

150

140

130

120

110

100

90

80

60

50

40

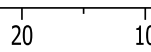


రับ

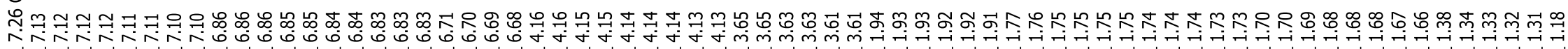

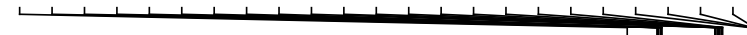

${ }^{1} \mathrm{H}-\mathrm{NMR}\left(600 \mathrm{MHz}, \mathrm{CDCl}_{3}\right)$ of $\mathbf{3 g}$

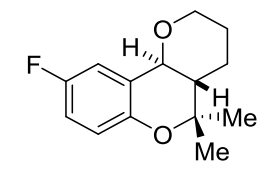

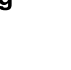




$$
\text { 告 }
$$

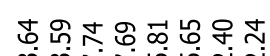

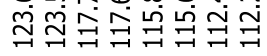

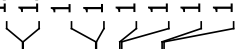

\section{응}

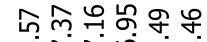

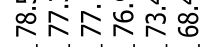

।

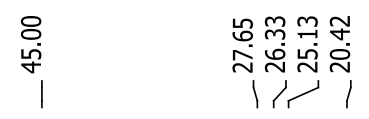

${ }^{13} \mathrm{C}-\mathrm{NMR}\left(150 \mathrm{MHz}, \mathrm{CDCl}_{3}\right.$ ) of $\mathbf{3 g}$
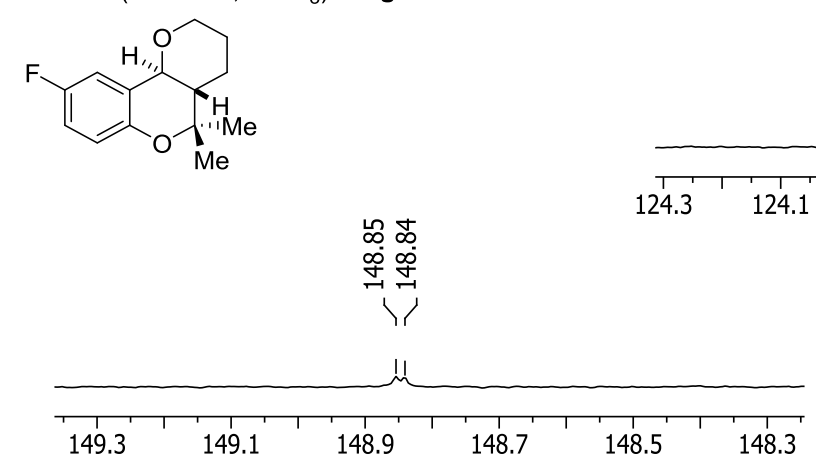

훙유

๗ㅋㅀำ

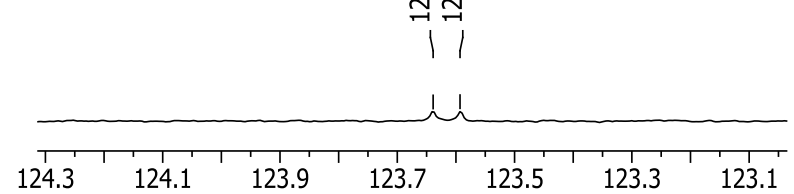

8.3

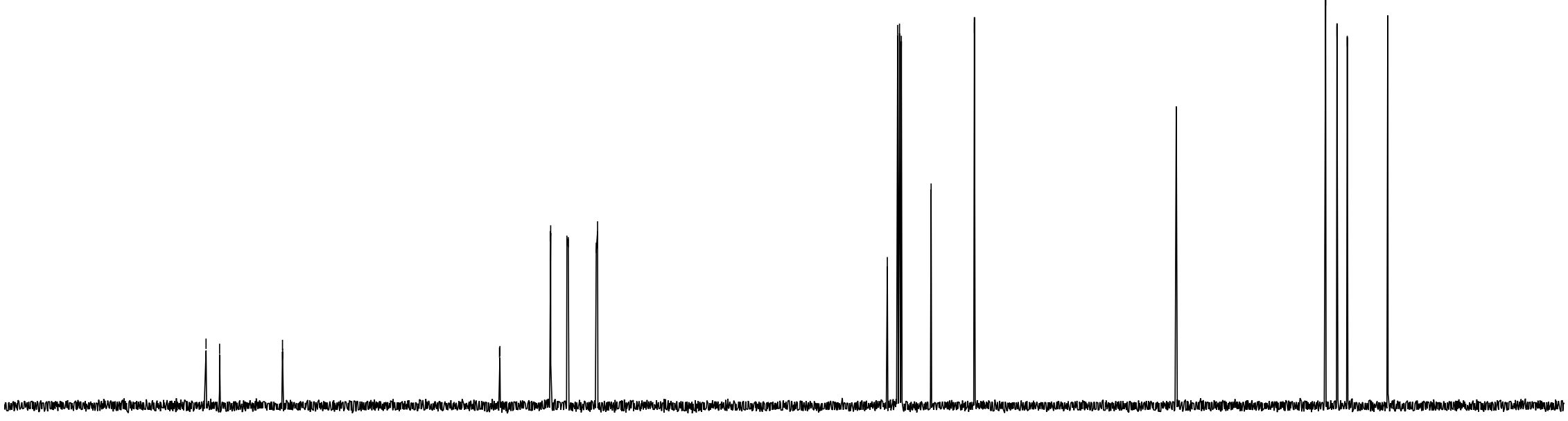

$\frac{7}{180}$

150

140

130

120

$110 \quad 100$

90

80

|

辛
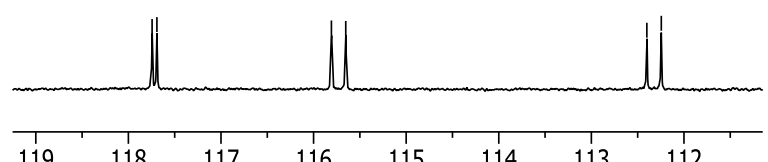

119

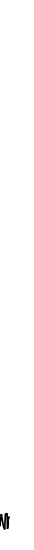




\section{${ }^{19} \mathrm{~F}-\mathrm{NMR}\left(565 \mathrm{MHz}, \mathrm{CDCl}_{3}\right)$ of $\mathbf{3 g}$}
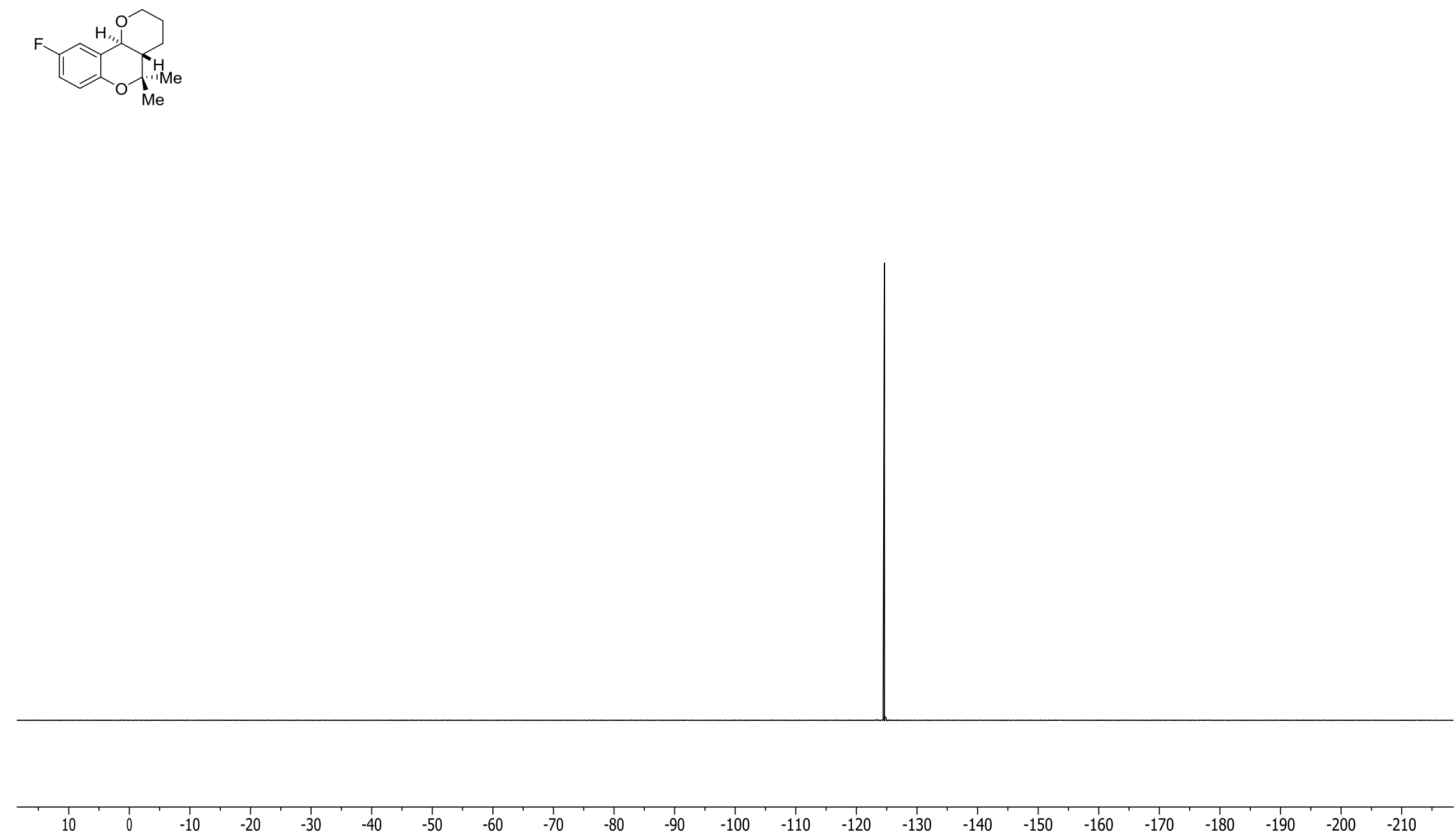
荅

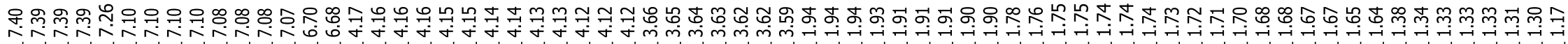

${ }^{1} \mathrm{H}-\mathrm{NMR}\left(400 \mathrm{MHz}, \mathrm{CDCl}_{3}\right)$ of $\mathbf{3 h}$<smiles>CC1(C)Oc2ccc(Cl)cc2[C@@H]2OCCC[C@]21C</smiles>

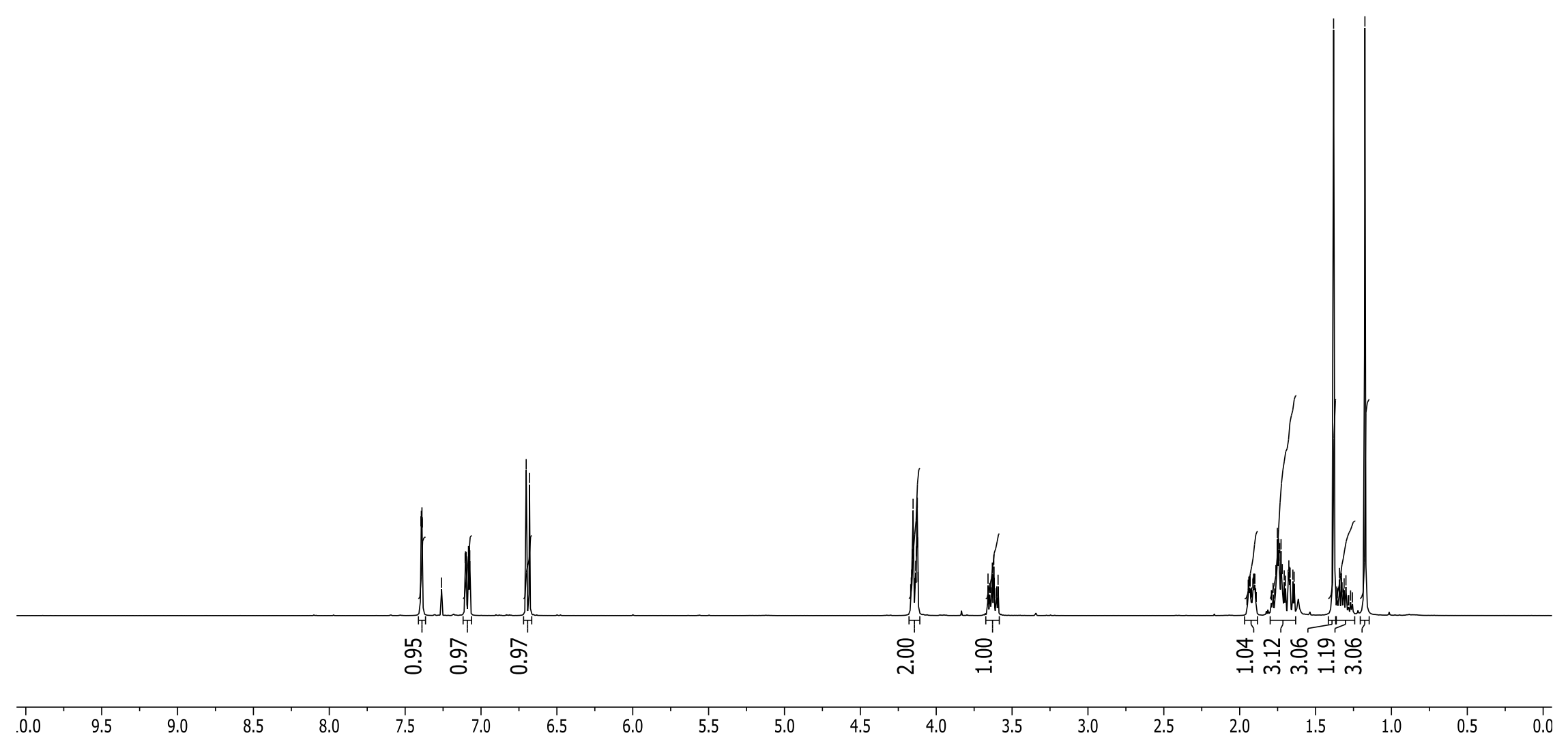




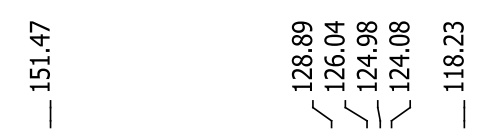
응 응

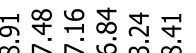
パト゚パ
।

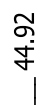

융ํㅇำ

귯ำ

${ }^{13} \mathrm{C}-\mathrm{NMR}\left(100 \mathrm{MHz}, \mathrm{CDCl}_{3}\right.$ ) of $\mathbf{3 h}$
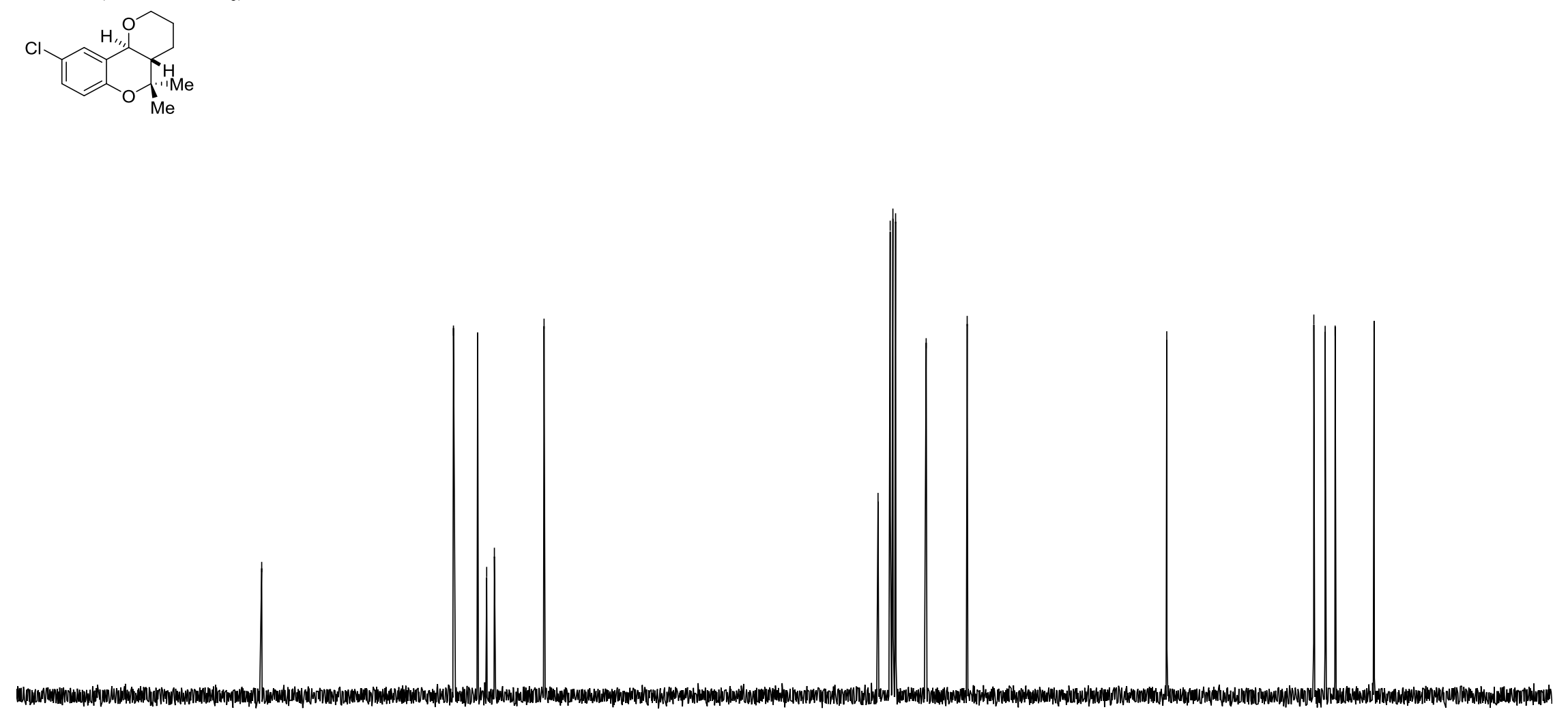

80

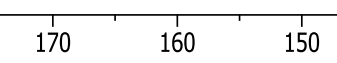

$140 \quad 130$

120

110

100

90

80

$70 \quad 60$

50

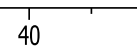

$30 \quad 20 \quad 10$ 


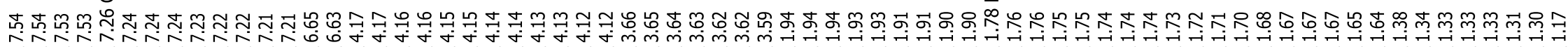

${ }^{1} \mathrm{H}-\mathrm{NMR}\left(400 \mathrm{MHz}, \mathrm{CDCl}_{3}\right)$ of $\mathbf{3 i}$
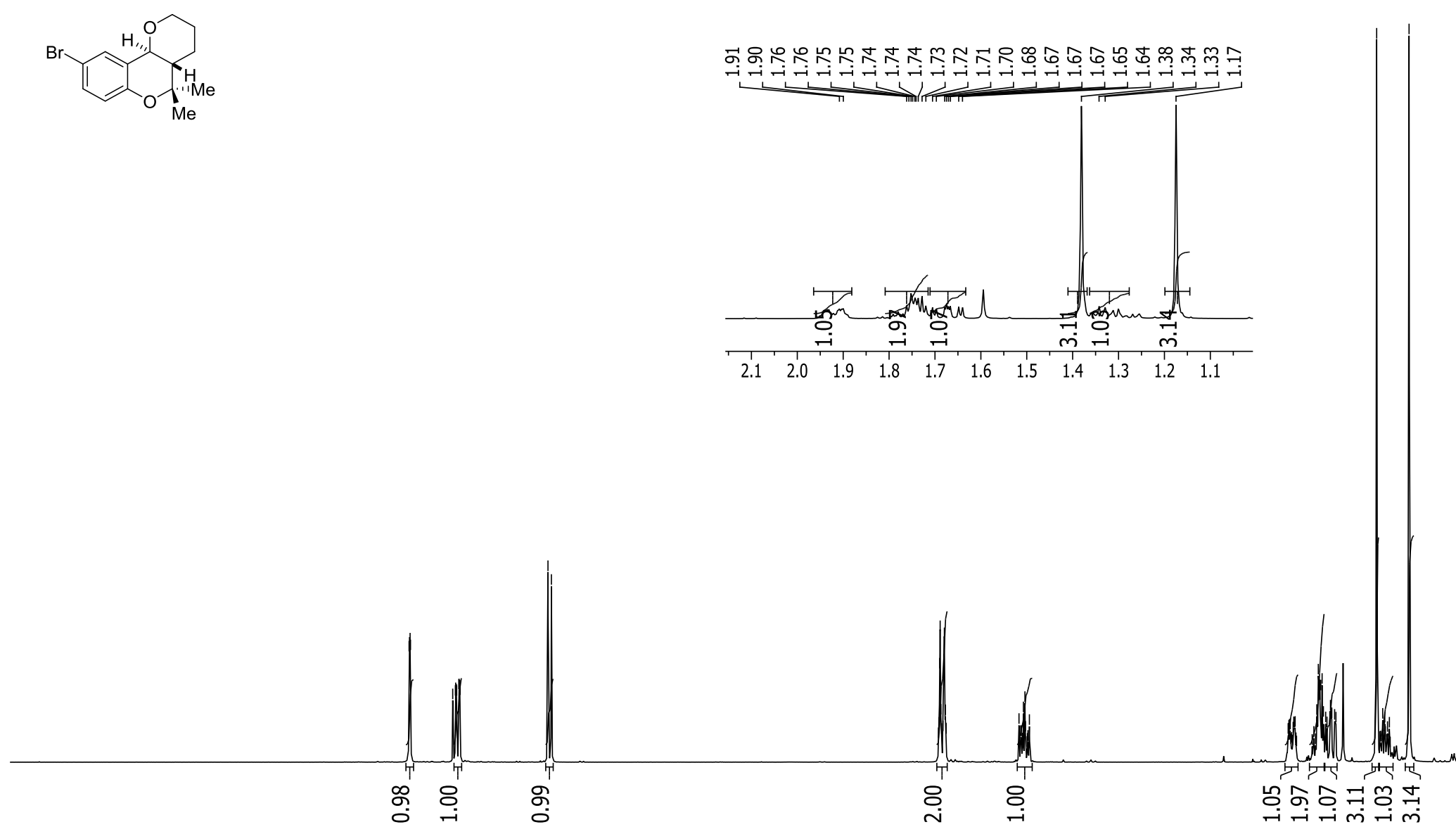


每

${ }^{13} \mathrm{C}-\mathrm{NMR}\left(100 \mathrm{MHz}, \mathrm{CDCl}_{3}\right.$ ) of $3 \mathrm{i}$
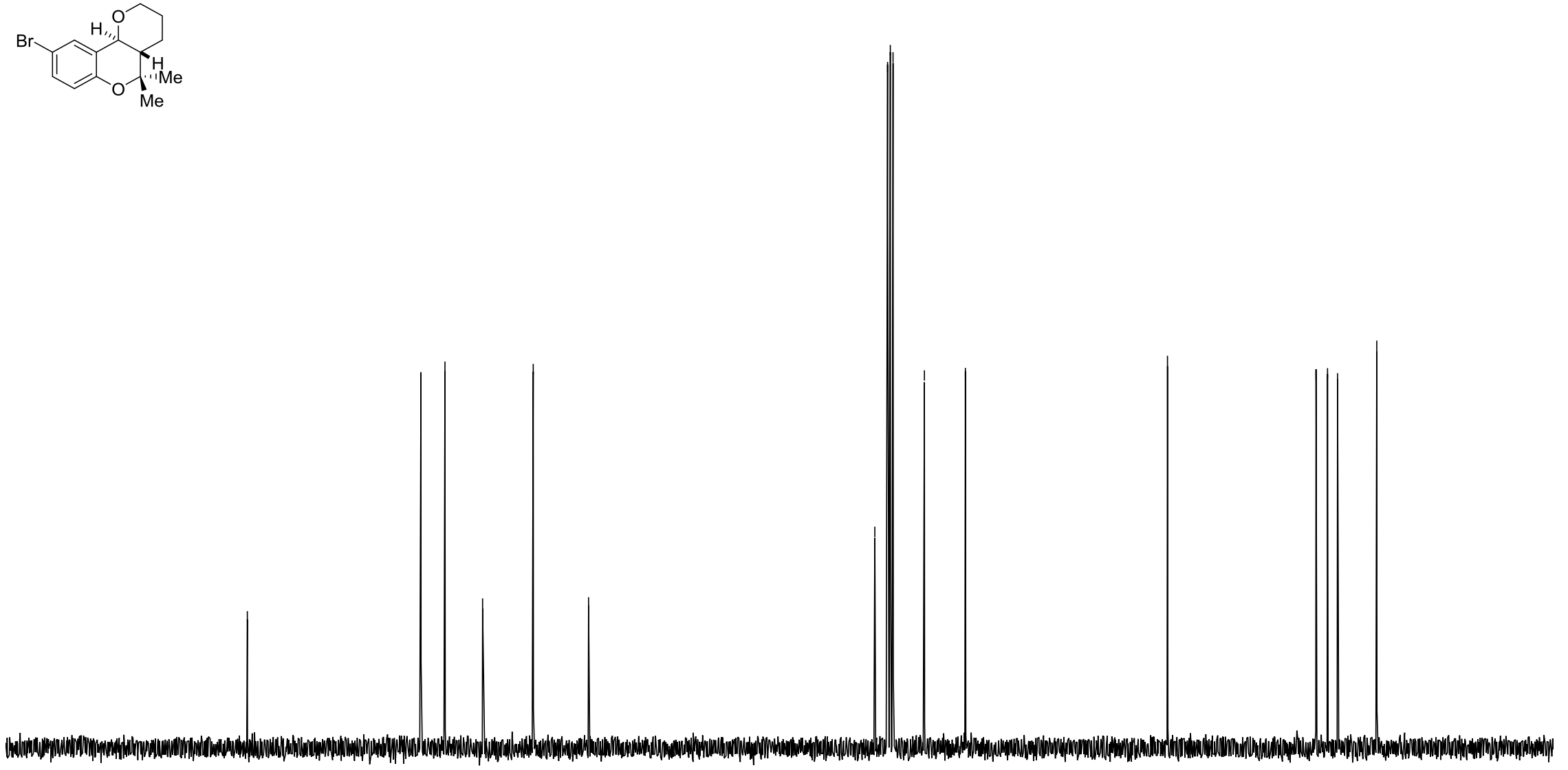

$$
30
$$

$160+150$

140

130

120

110

100

90

80

70

60

$50 \quad 40$

$30 \quad 20$

10 
范

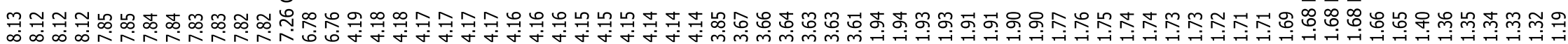

${ }^{1} \mathrm{H}-\mathrm{NMR}\left(400 \mathrm{MHz}, \mathrm{CDCl}_{3}\right)$ of $\mathbf{3 j}$
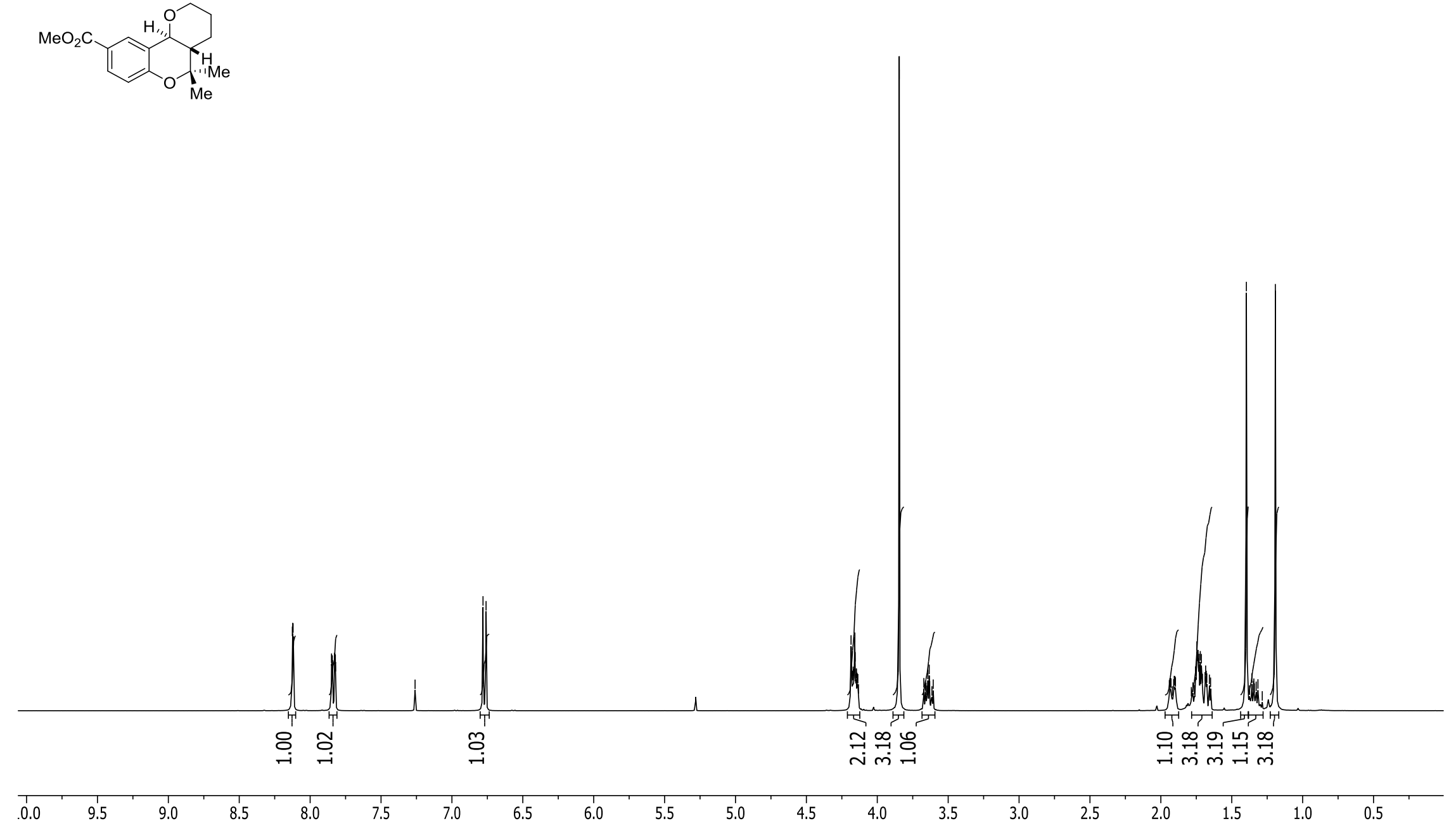

8.0

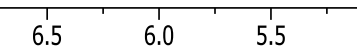




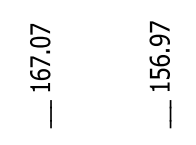

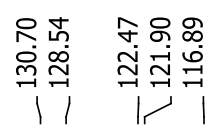

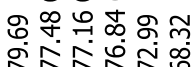

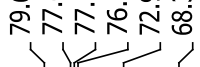

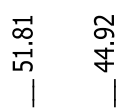

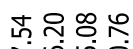
긴

응

${ }^{13} \mathrm{C}-\mathrm{NMR}\left(100 \mathrm{MHz}, \mathrm{CDCl}_{3}\right)$ of $\mathbf{3 j}$
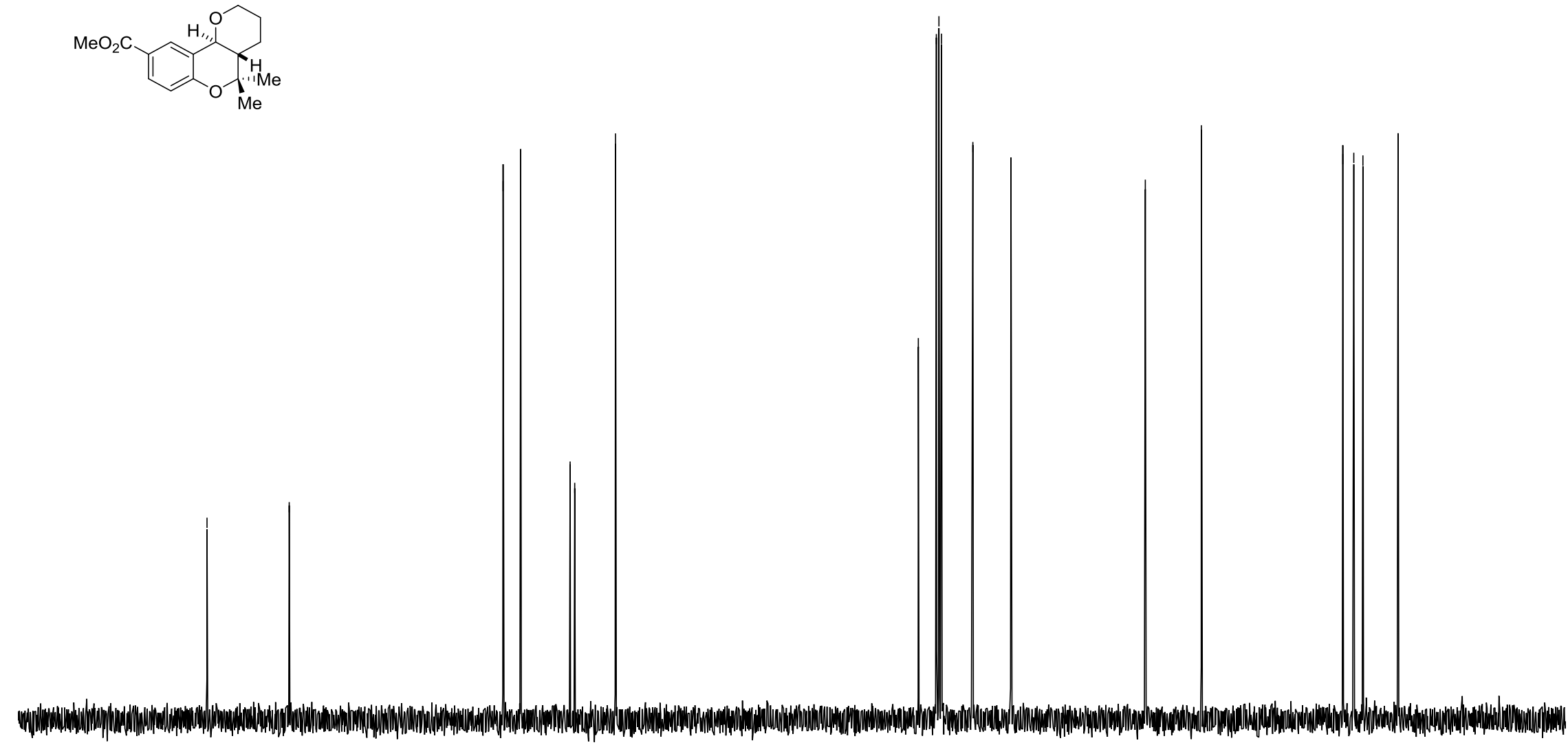

$$
90
$$




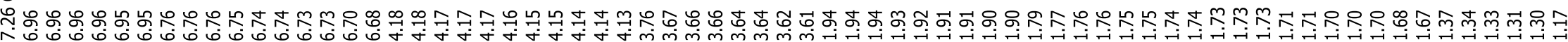

${ }^{1} \mathrm{H}-\mathrm{NMR}\left(400 \mathrm{MHz}, \mathrm{CDCl}_{3}\right)$ of $\mathbf{3 k}$

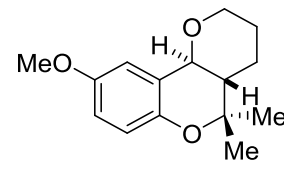

$\mathrm{Me}$

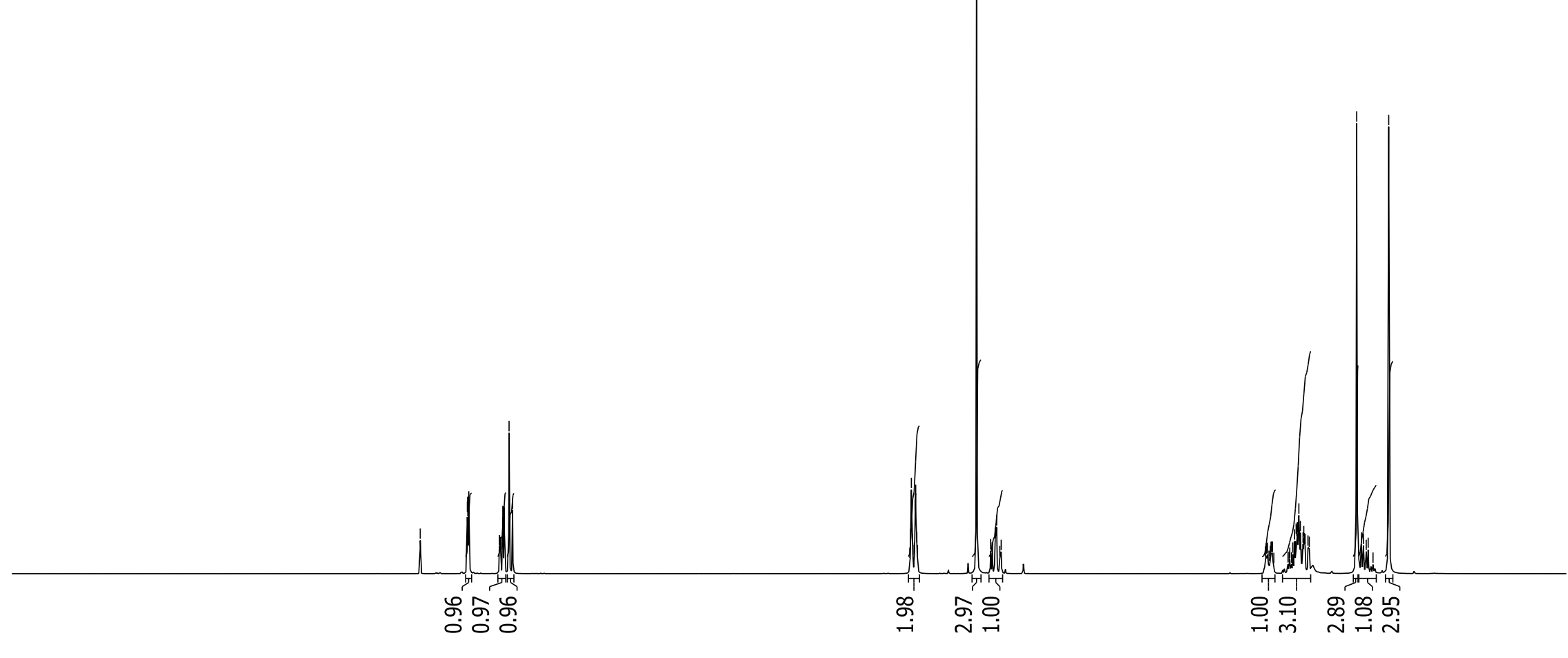

8.5

8.0

6.5

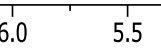

5.0

$4.0 \quad 3.5$

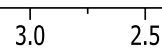




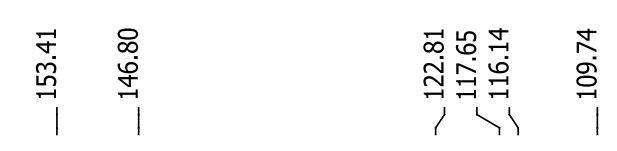

$$
\begin{aligned}
& \text { 豕商商 }
\end{aligned}
$$

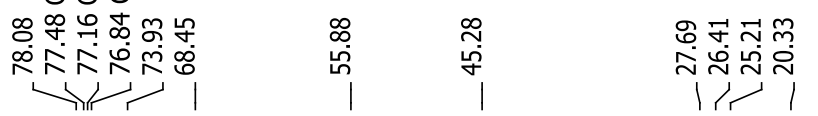

${ }^{13} \mathrm{C}-\mathrm{NMR}\left(100 \mathrm{MHz}, \mathrm{CDCl}_{3}\right)$ of $\mathbf{3 k}$
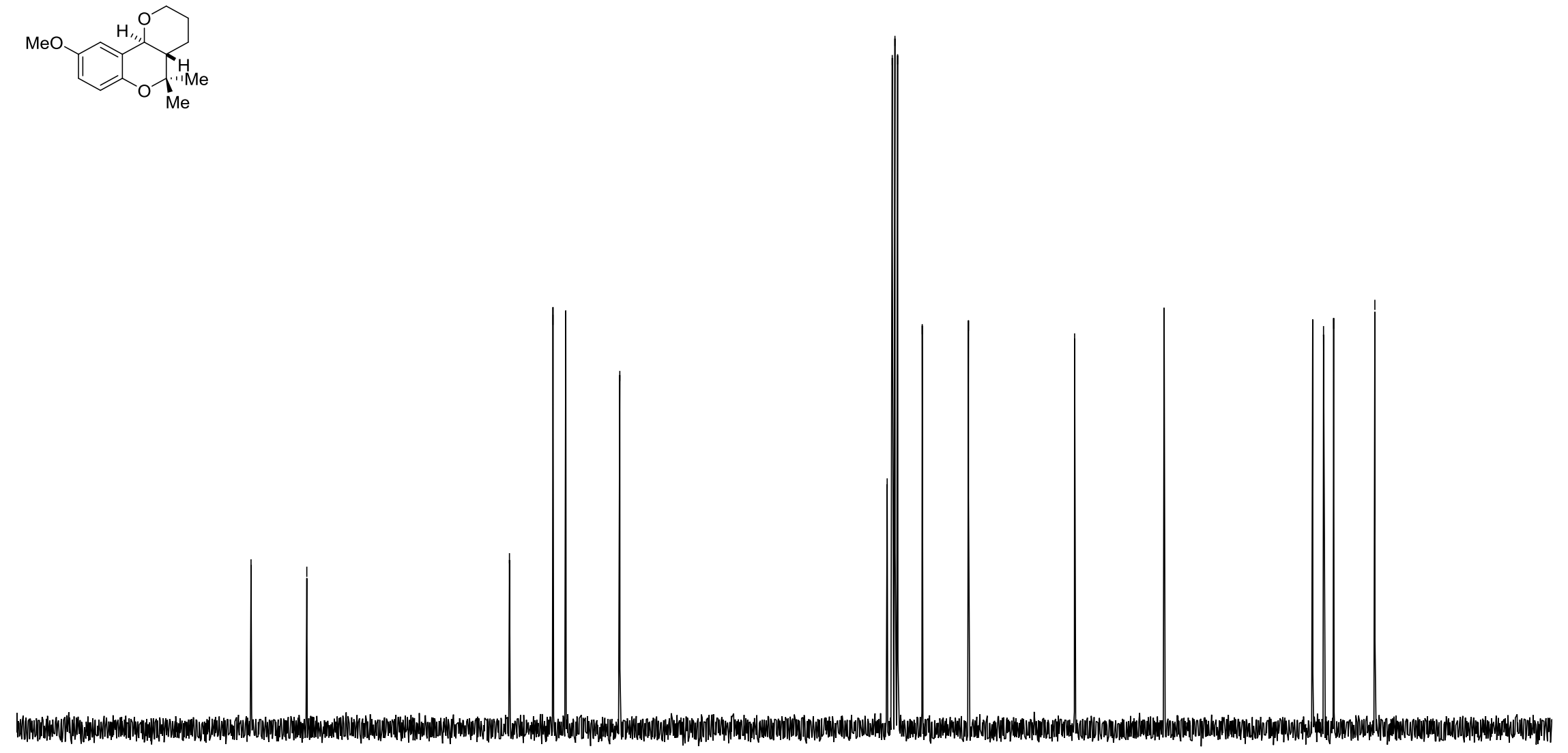

$$
\frac{1}{180}
$$

170

160

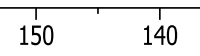

$130 \div \quad 120$

$110 \quad 100$

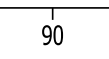

80

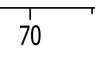

60

50

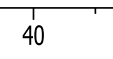

30

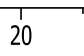

10 


\section{愛}

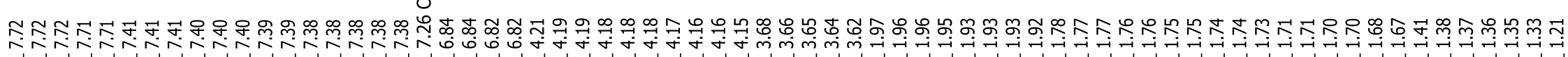

${ }^{1} \mathrm{H}-\mathrm{NMR}\left(400 \mathrm{MHz}, \mathrm{CDCl}_{3}\right)$ of $\mathbf{3 l}$
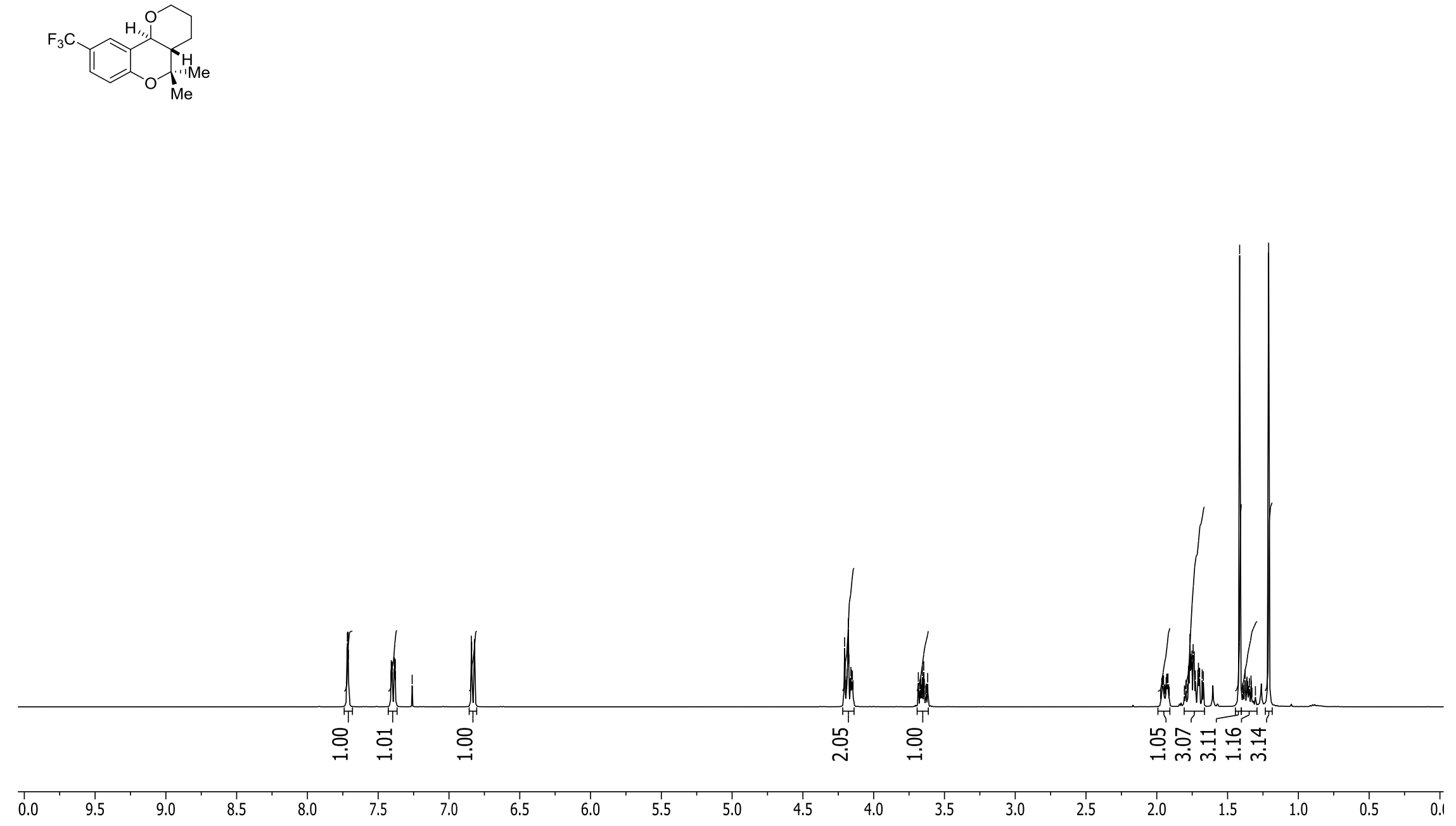


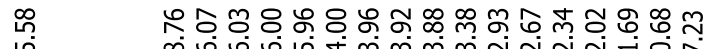

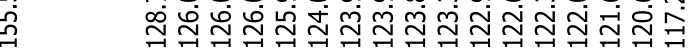

${ }^{13} \mathrm{C}-\mathrm{NMR}\left(100 \mathrm{MHz}, \mathrm{CDCl}_{3}\right)$ of 31

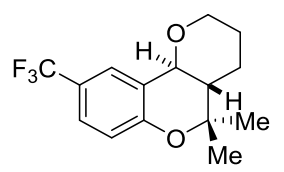

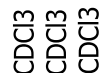

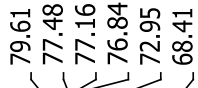

lit?

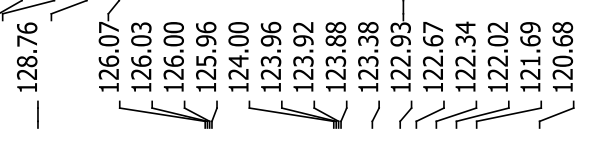

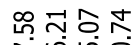

กั่ำ่

liti

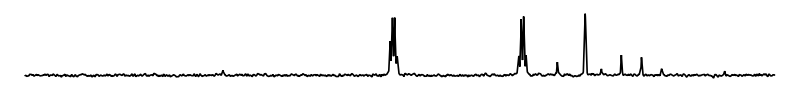

$\begin{array}{lllllllllllllll}131 & 130 & 129 & 128 & 127 & 126 & 125 & 124 & 123 & 122 & 121 & 120\end{array}$

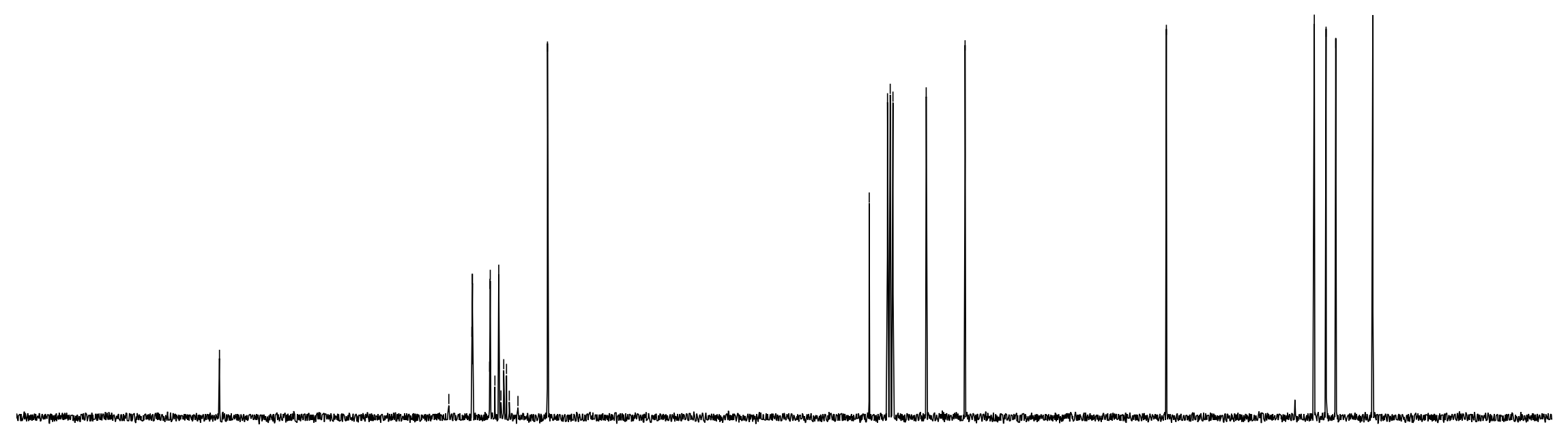

S-155 
${ }^{19} \mathrm{~F}-\mathrm{NMR}\left(565 \mathrm{MHz}, \mathrm{CDCl}_{3}\right)$ of $3 \mathbf{l}$

$$
\text { (1) }
$$
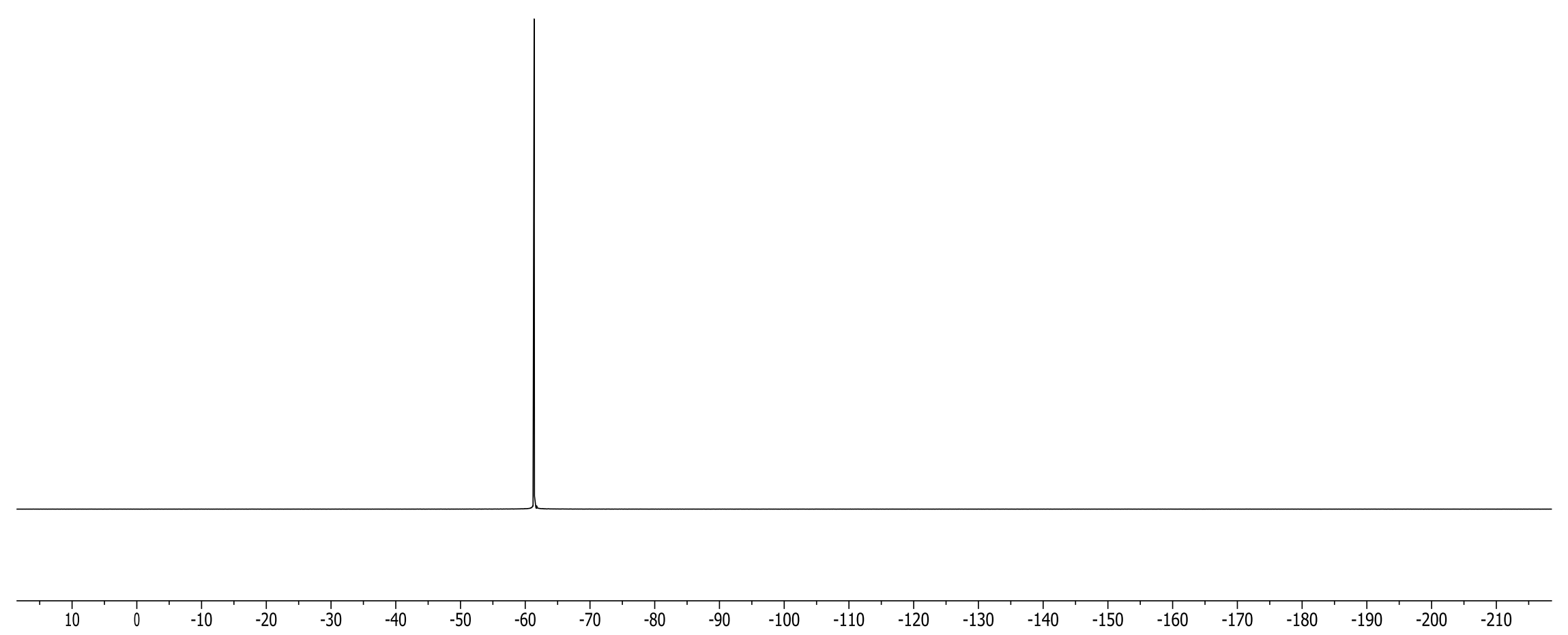


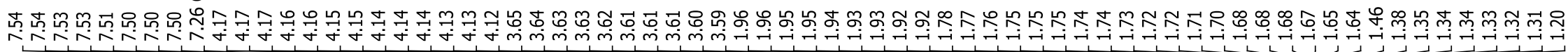

${ }^{1} \mathrm{H}-\mathrm{NMR}\left(400 \mathrm{MHz}, \mathrm{CDCl}_{3}\right)$ of $\mathbf{3 m}$
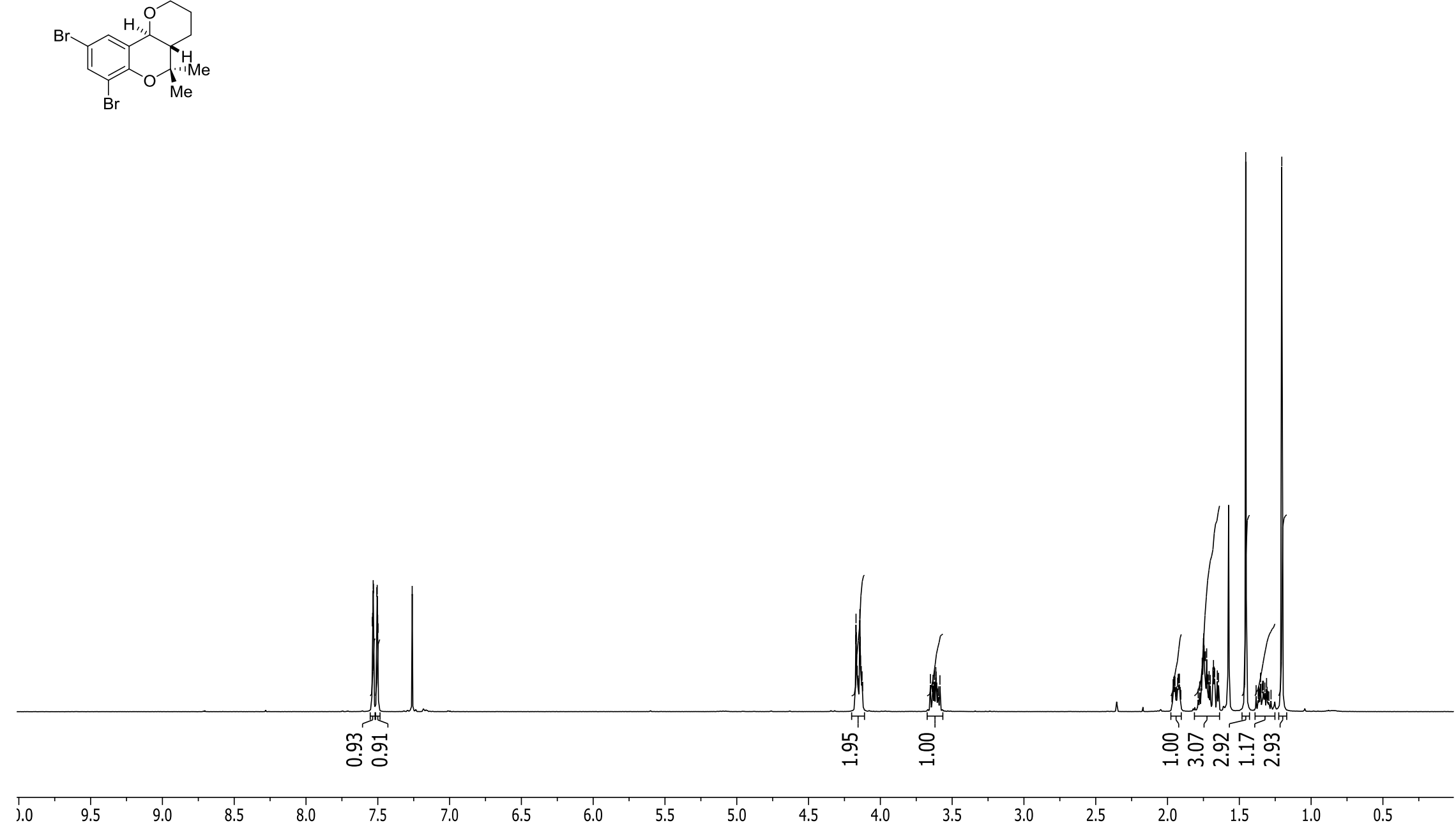


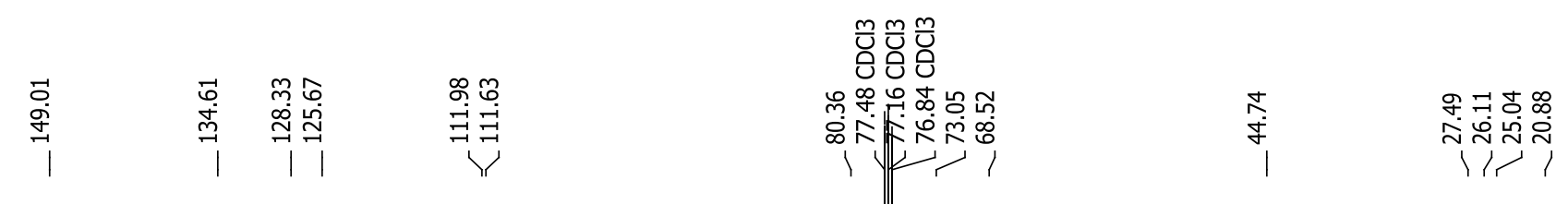

${ }^{13} \mathrm{C}-\mathrm{NMR}\left(100 \mathrm{MHz}, \mathrm{CDCl}_{3}\right)$ of $\mathbf{3 m}$

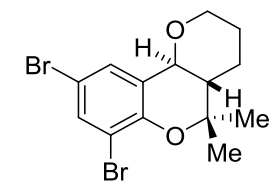

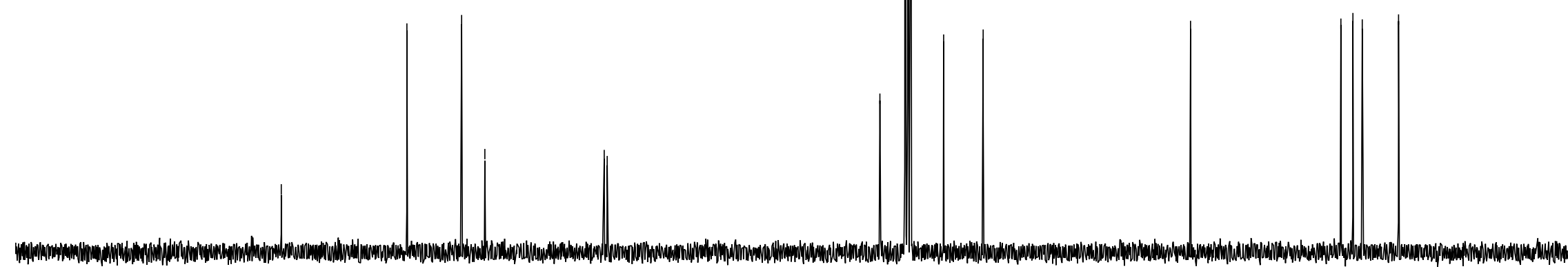




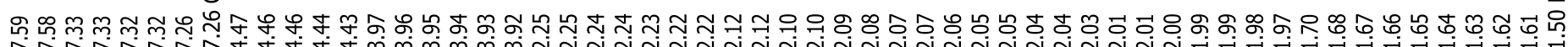

${ }^{1} \mathrm{H}-\mathrm{NMR}\left(600 \mathrm{MHz}, \mathrm{CDCl}_{3}\right)$ of $3 \mathrm{n}$

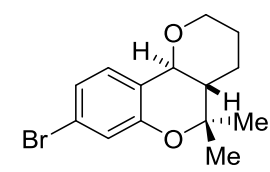

Me
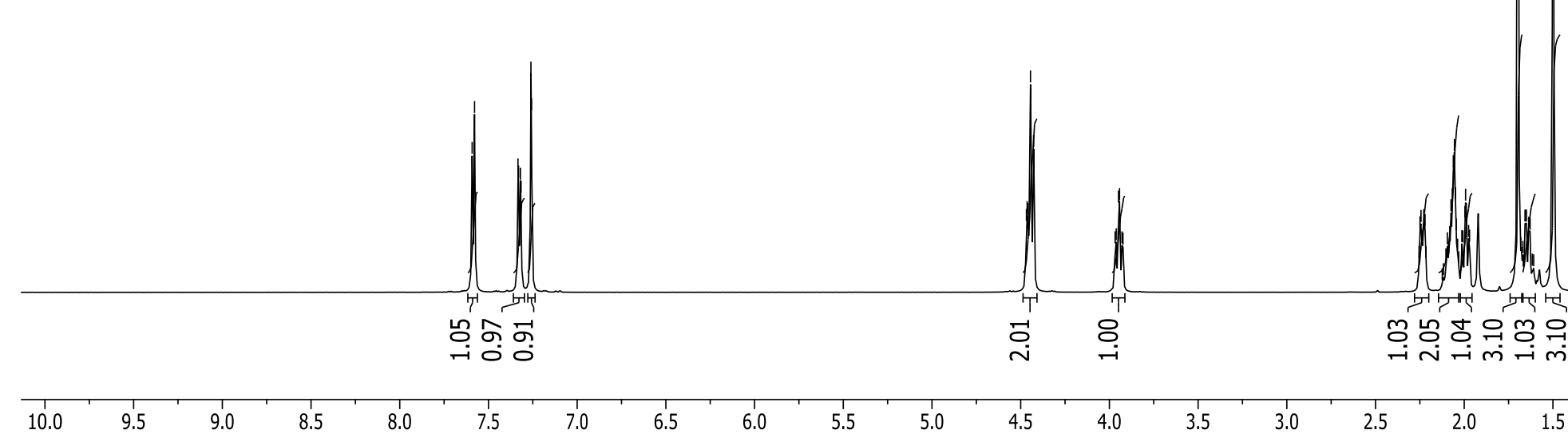

5.5 


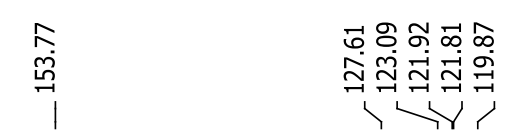

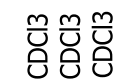

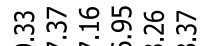

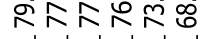

।

官

웅ำ 응

iाiti

${ }^{13} \mathrm{C}-\mathrm{NMR}\left(150 \mathrm{MHz}, \mathrm{CDCl}_{3}\right)$ of $\mathbf{3 n}$
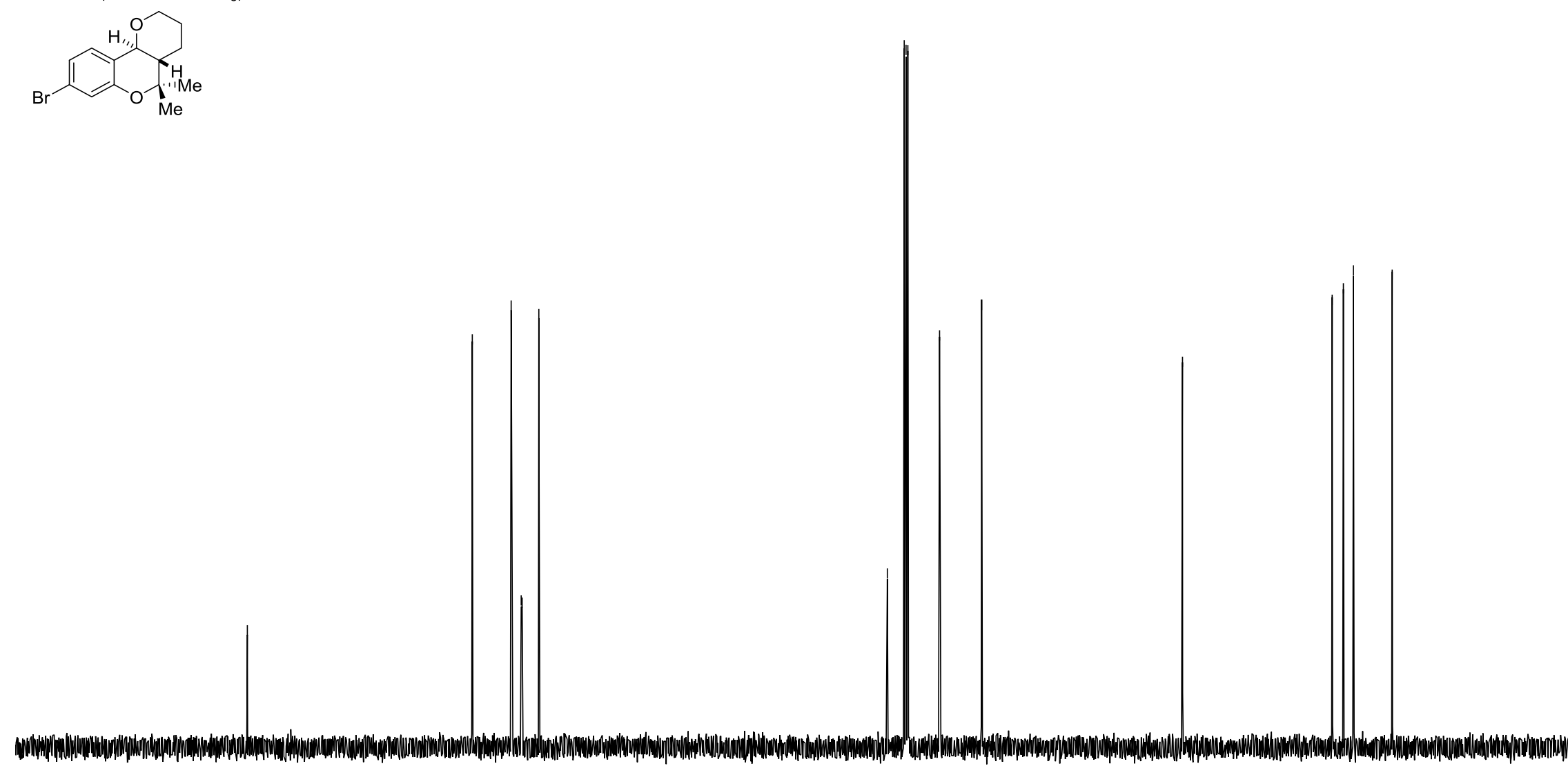

80

160

150

140

130

120

110

100

90

80

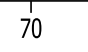

60

50

40

30

20 


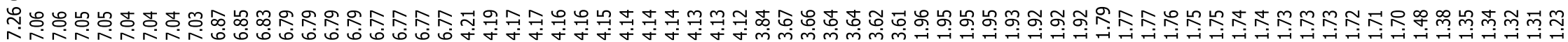
垈

${ }^{1} \mathrm{H}-\mathrm{NMR}\left(400 \mathrm{MHz}, \mathrm{CDCl}_{3}\right)$ of 30
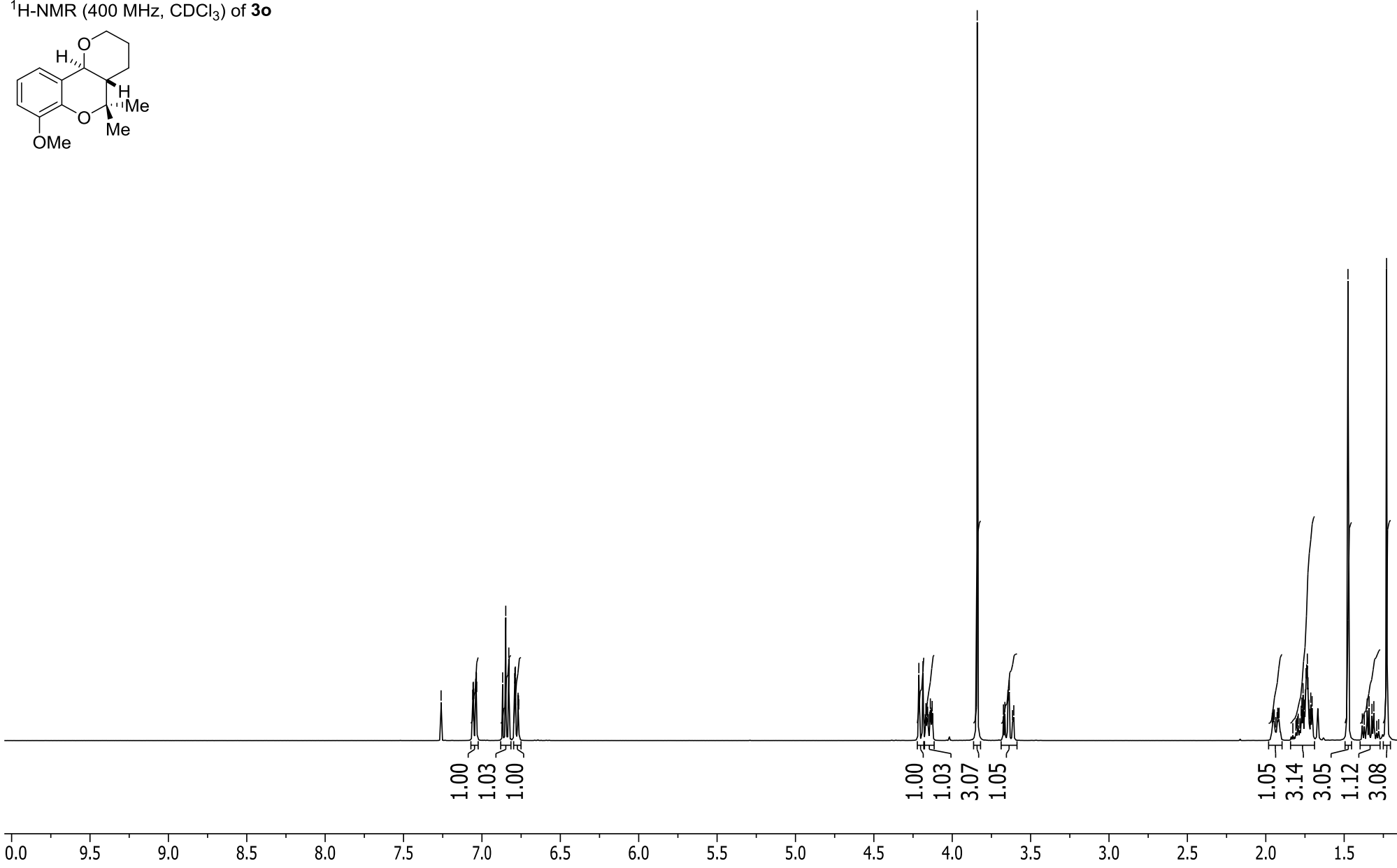

4.5

4.0

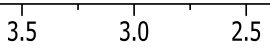

$2.0 \quad 1.5$ 


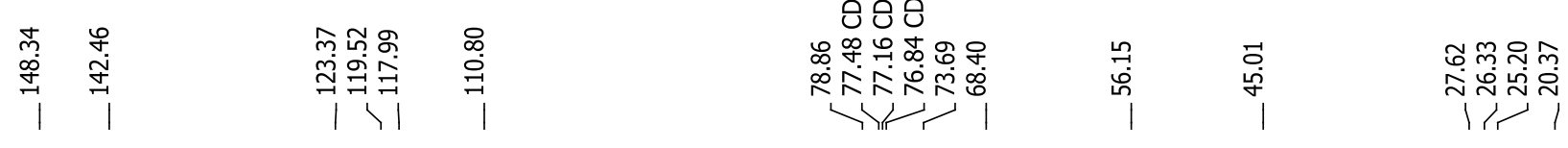

${ }^{13} \mathrm{C}-\mathrm{NMR}\left(100 \mathrm{MHz}, \mathrm{CDCl}_{3}\right)$ of $\mathbf{3 0}$
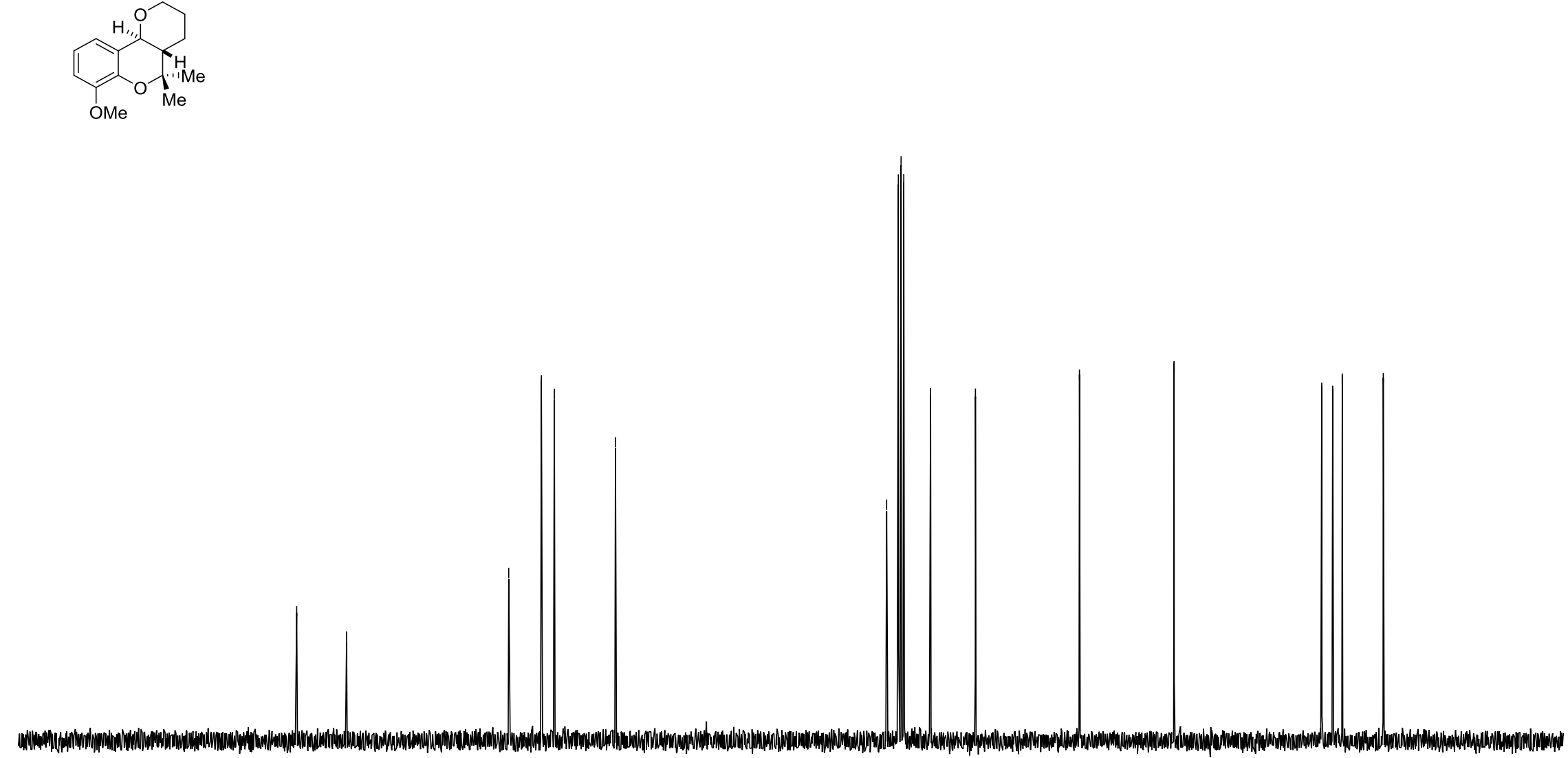

$170 \quad 160$

150

140

$130 \quad 120$

110

100

90

80

70

60

50

40

30

20

10 


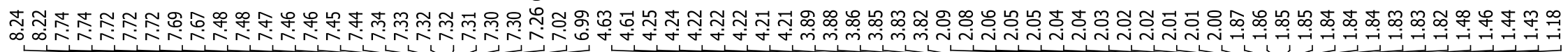

${ }^{1} \mathrm{H}-\mathrm{NMR}\left(400 \mathrm{MHz}, \mathrm{CDCl}_{3}\right)$ of $\mathbf{3 p}$
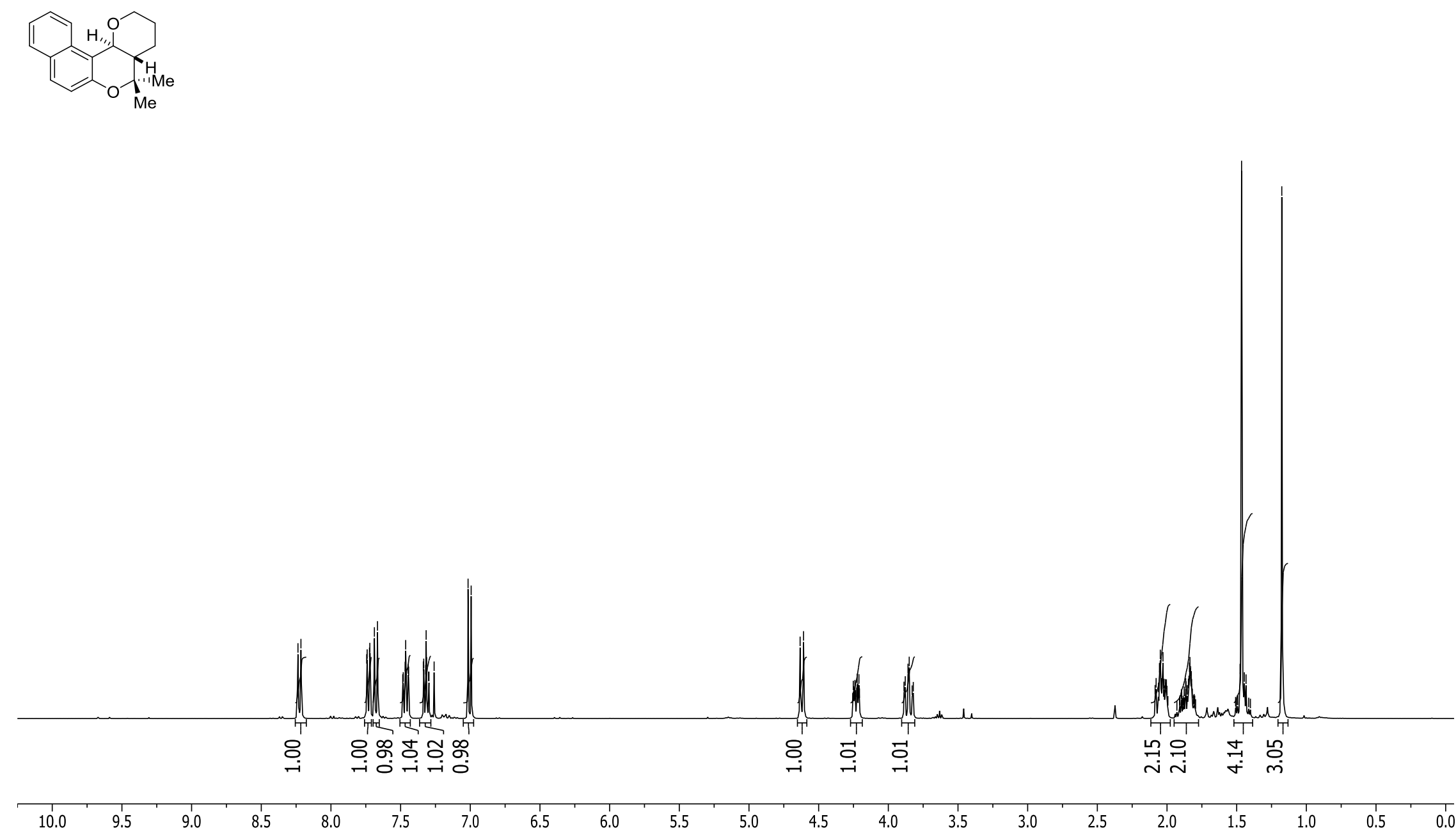


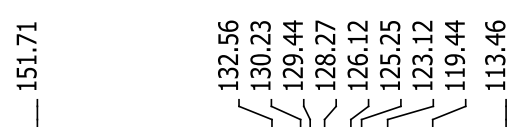

꽁ํํㅇำ

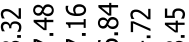

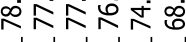

作।

8

m

ㄴ?

${ }^{13} \mathrm{C}-\mathrm{NMR}\left(100 \mathrm{MHz}, \mathrm{CDCl}_{3}\right.$ ) of $3 \mathbf{p}$

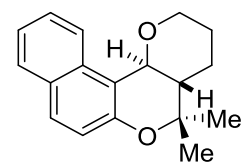

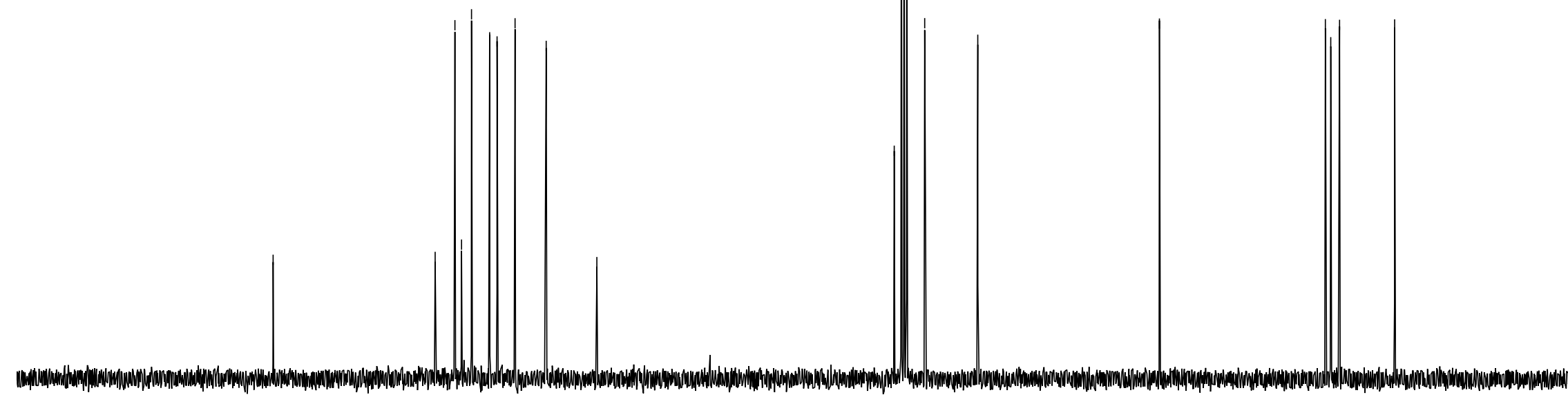

180

150

140

130

120

110

100

90

80

70

60

50

40

20

10 

in工

${ }^{1} \mathrm{H}-\mathrm{NMR}\left(500 \mathrm{MHz}, \mathrm{CDCl}_{3}\right)$ of 7
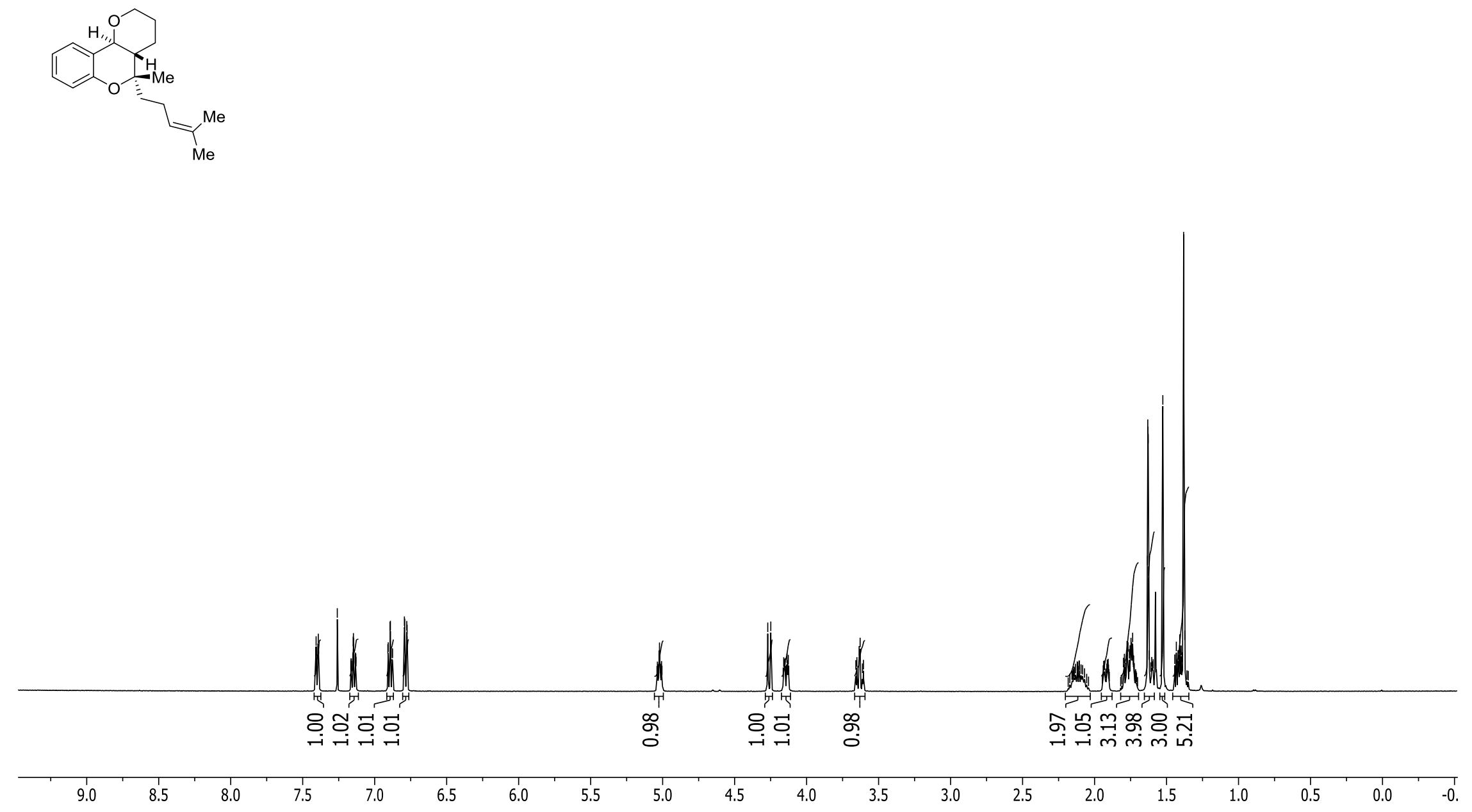


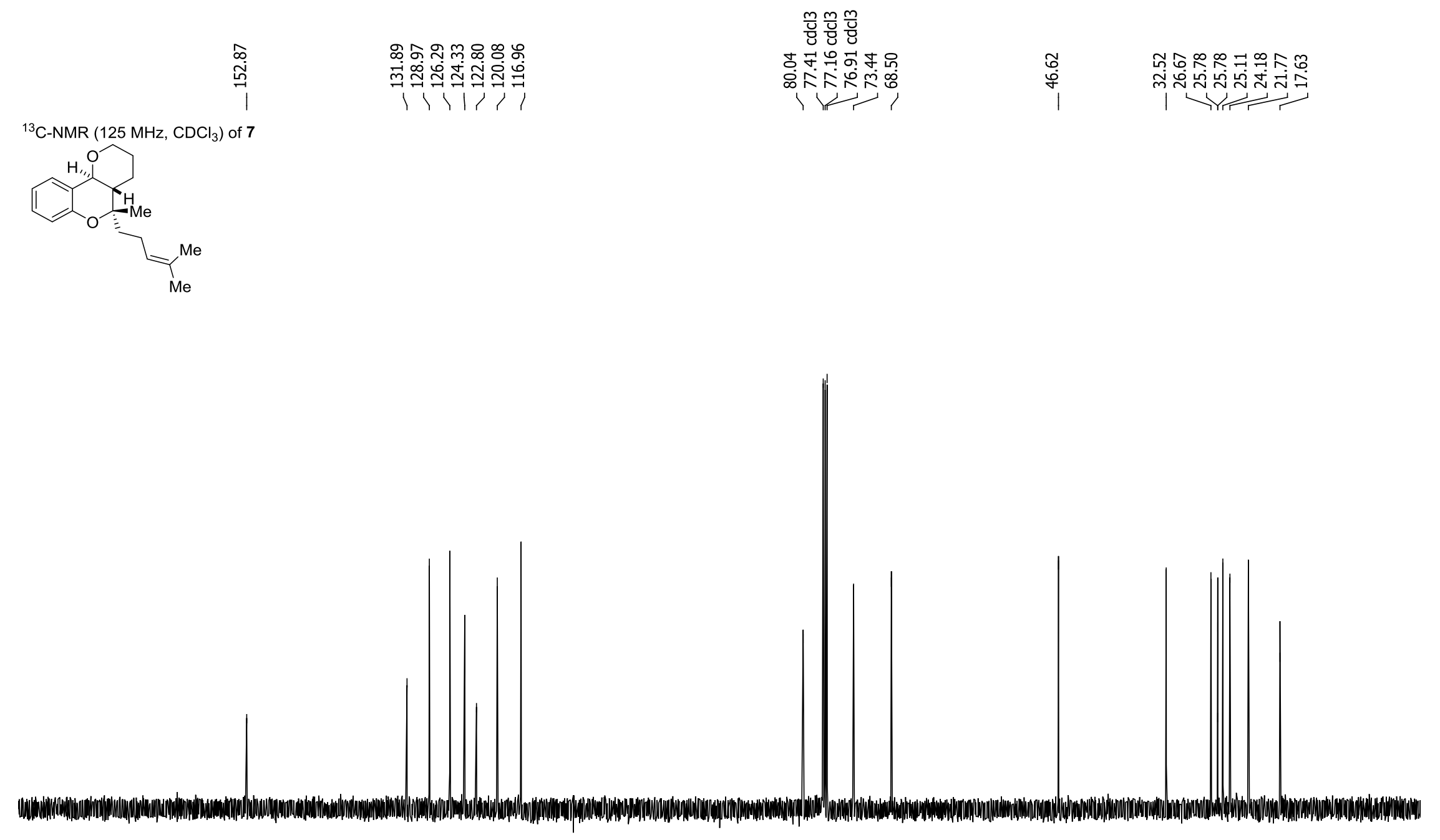

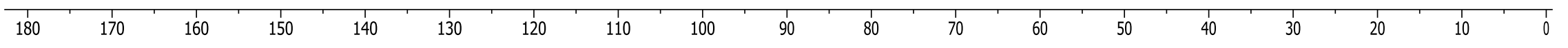




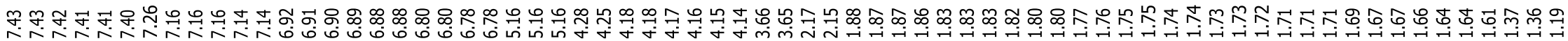

${ }^{1} \mathrm{H}-\mathrm{NMR}\left(400 \mathrm{MHz}, \mathrm{CDCl}_{3}\right)$ of 9

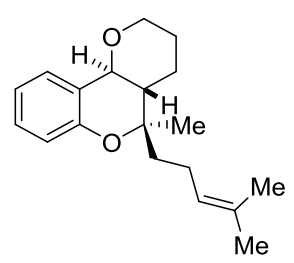

e

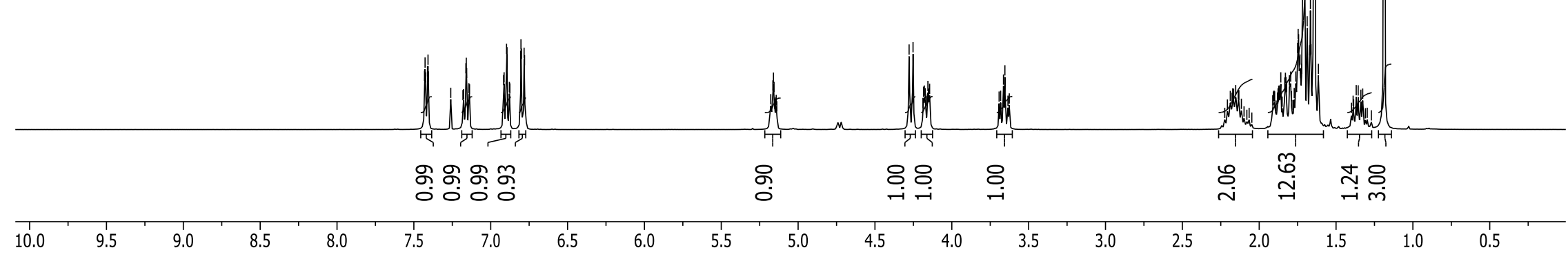




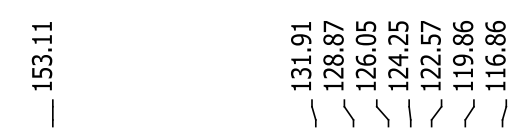

용ํำ

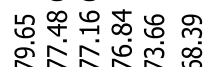

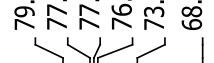

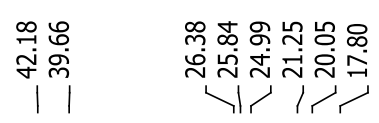

${ }^{13} \mathrm{C}-\mathrm{NMR}\left(100 \mathrm{MHz}, \mathrm{CDCl}_{3}\right)$ of 9
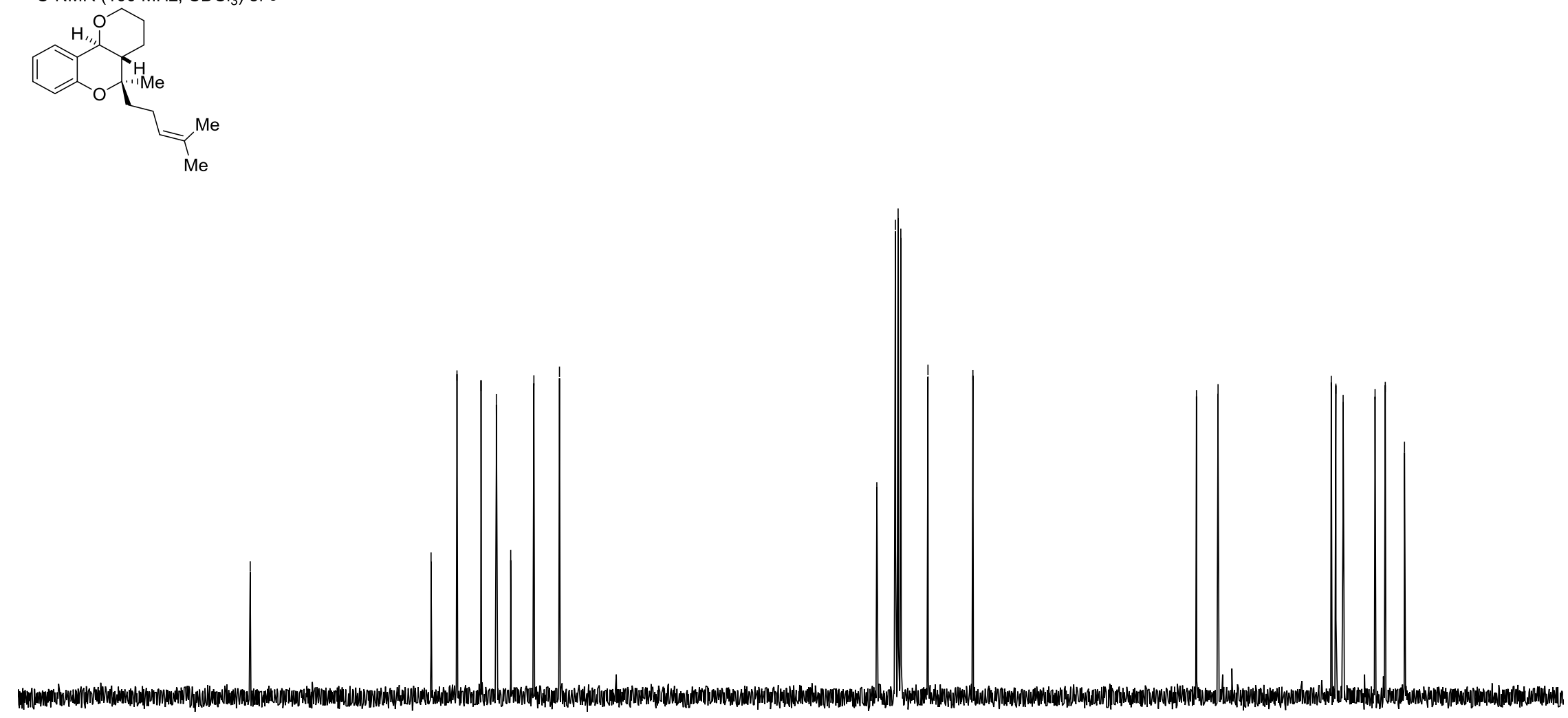

80 170 160 150 140 130 120 110 100 90 80 1
$70 \quad 60$ 50 40 30 20 10 


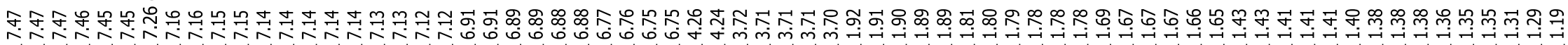

${ }^{1} \mathrm{H}-\mathrm{NMR}\left(500 \mathrm{MHz}, \mathrm{CDCl}_{3}\right)$ of 11
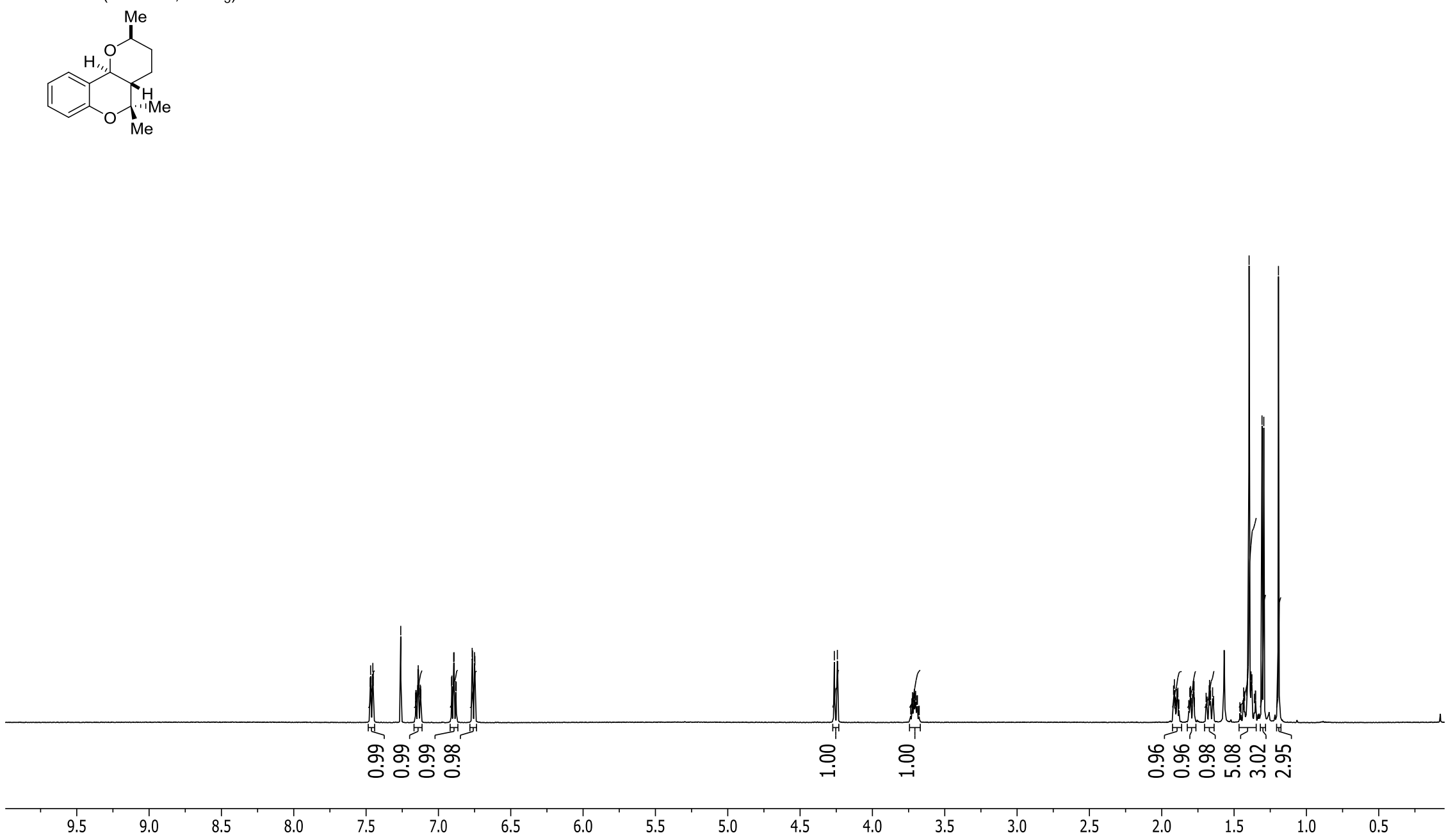


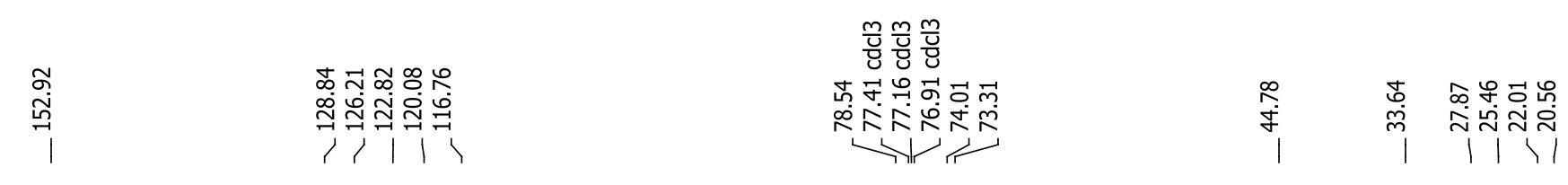
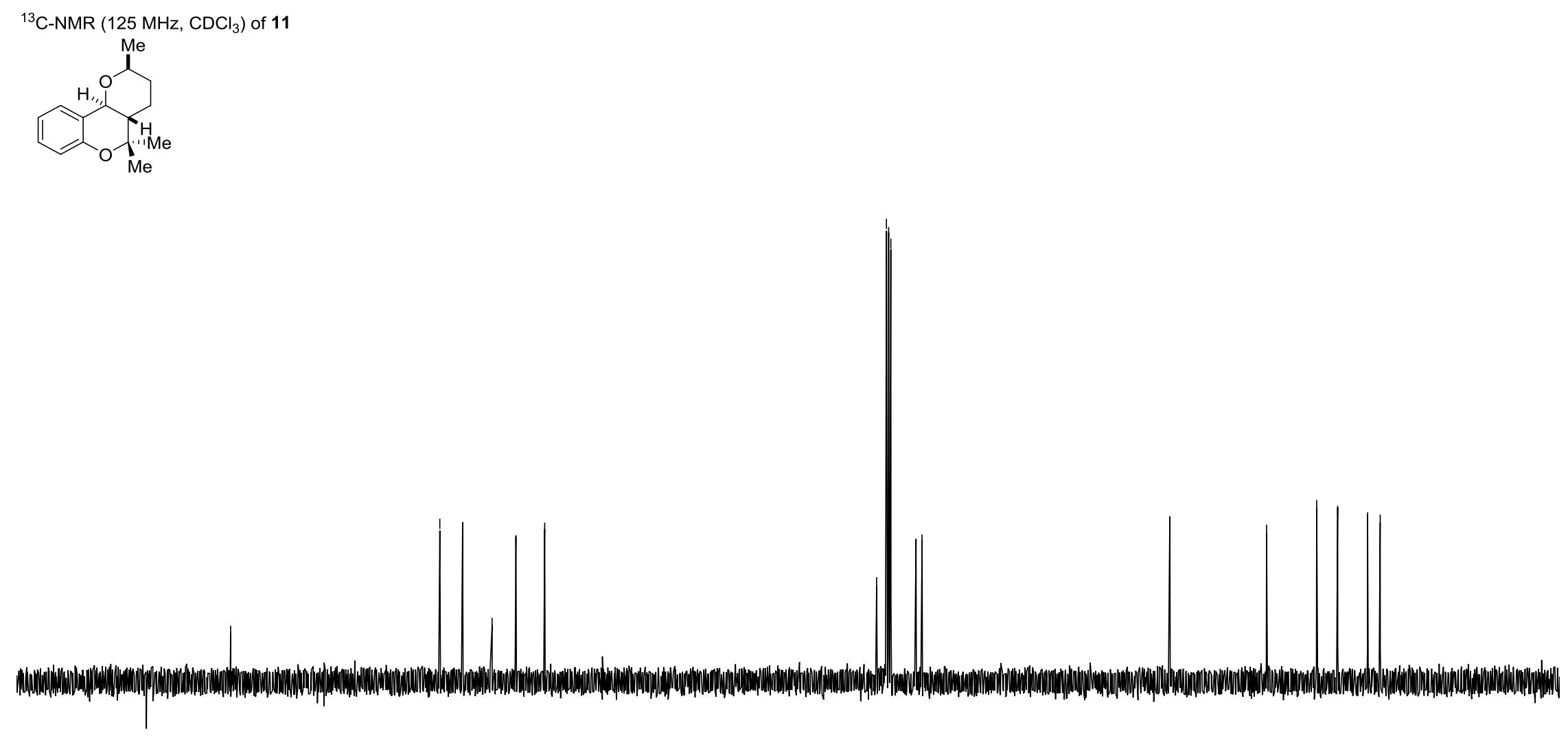

\begin{tabular}{llllllllllllllll}
\hline 170 & 160 & 150 & 140 & 130 & 120 & 110 & 100 & 90 & 80 & 70 & 60 & 50 & 40 & 1 & 1
\end{tabular}


产

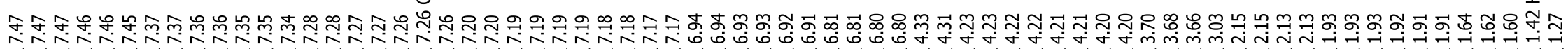

${ }^{1} \mathrm{H}-\mathrm{NMR}\left(600 \mathrm{MHz}, \mathrm{CDCl}_{3}\right)$ of 13
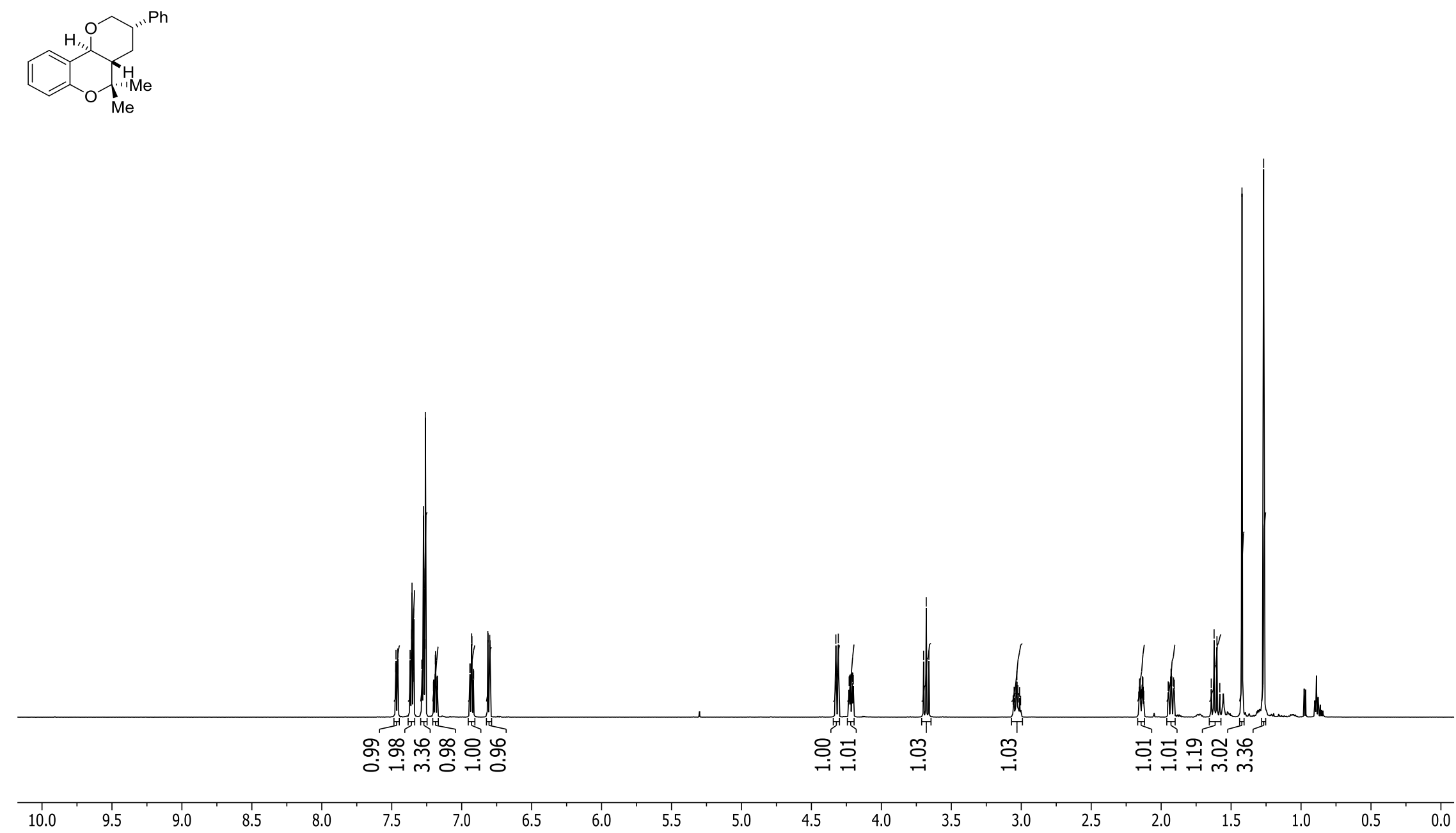
${ }^{13} \mathrm{C}-\mathrm{NMR}\left(150 \mathrm{MHz}, \mathrm{CDCl}_{3}\right)$ of 13
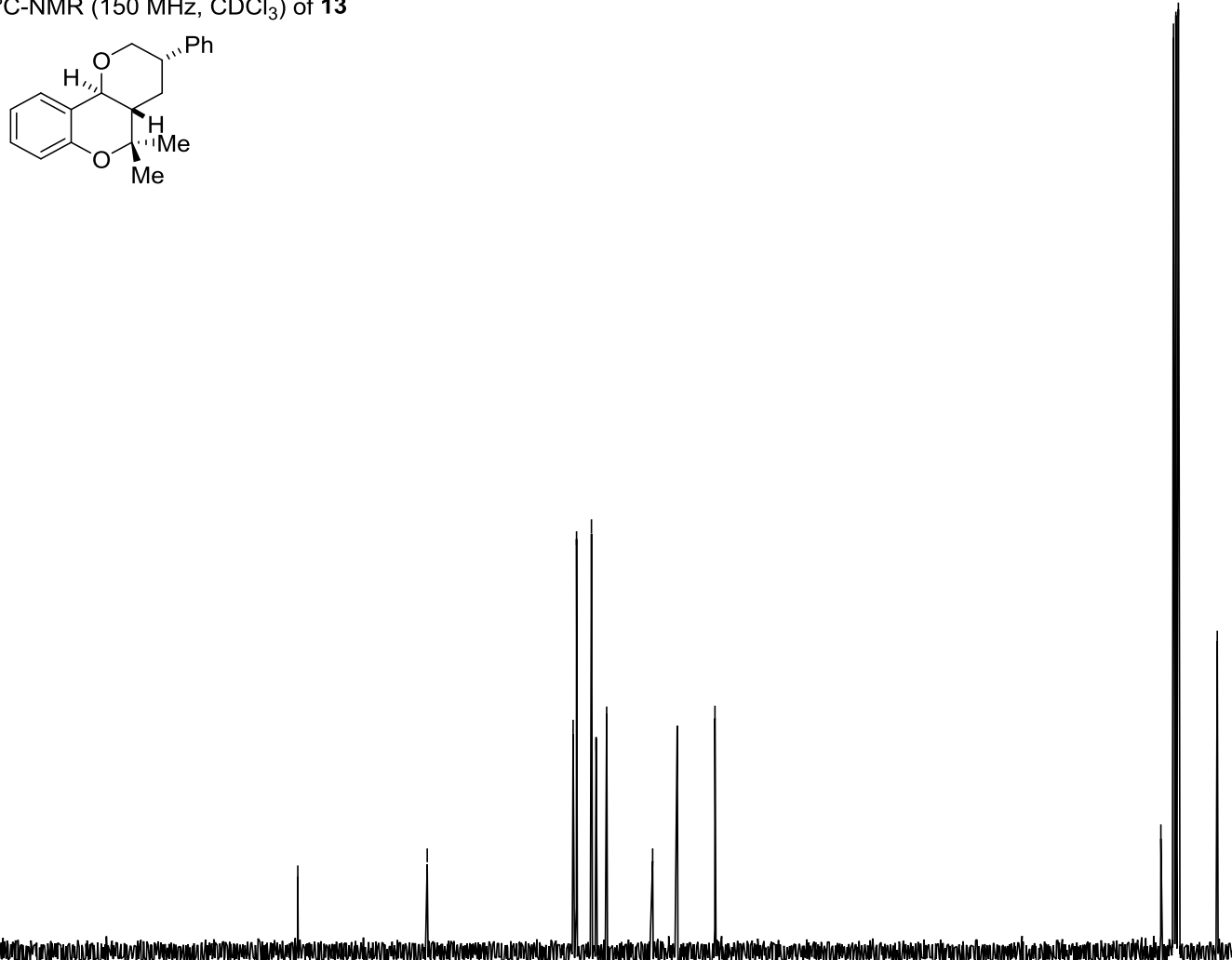

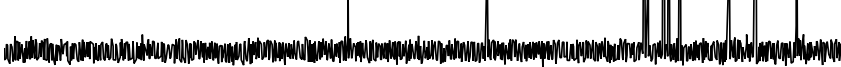


$\operatorname{GCOSY}\left(600 \mathrm{MHz}, \mathrm{CDCl}_{3}\right)$ of 13
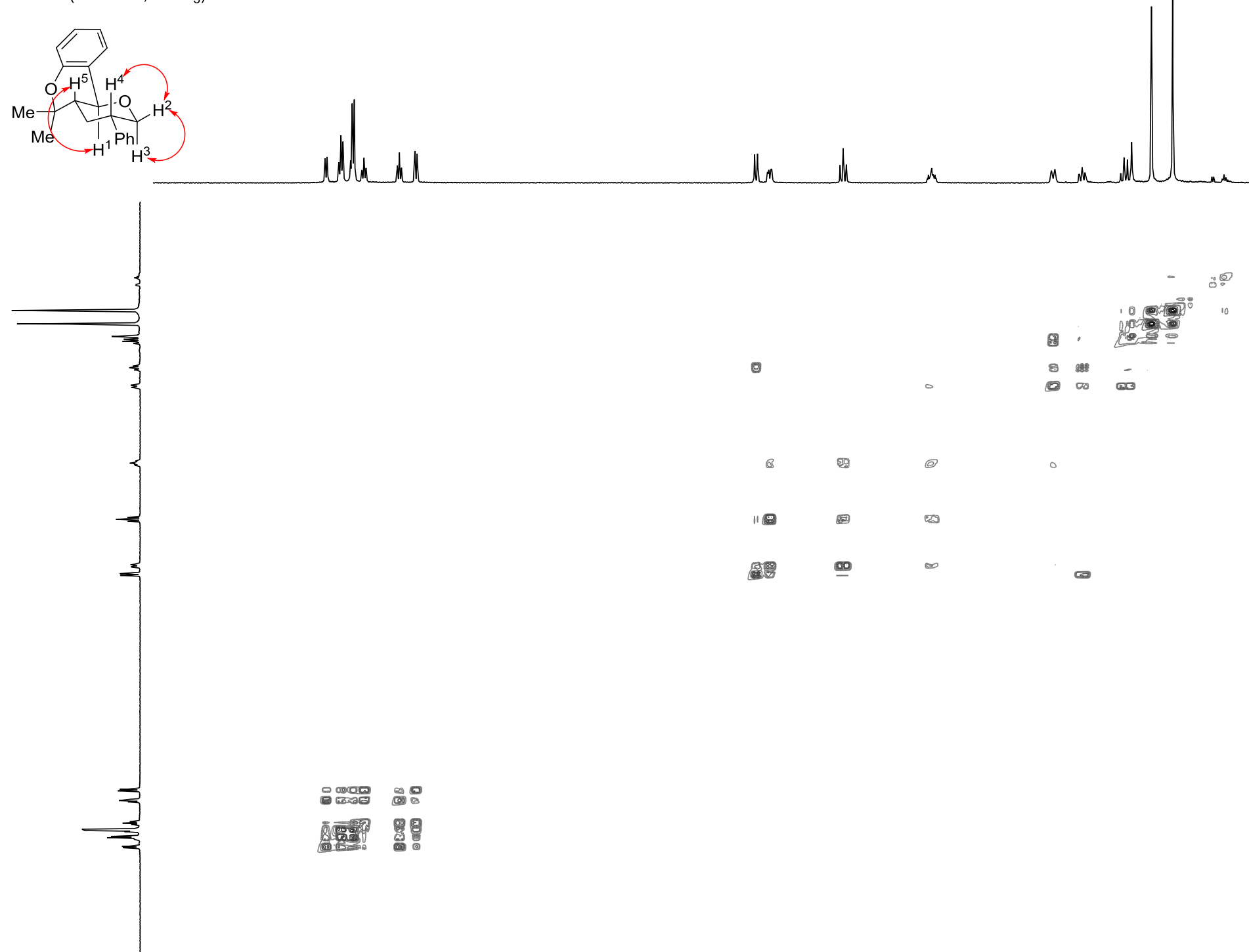

o

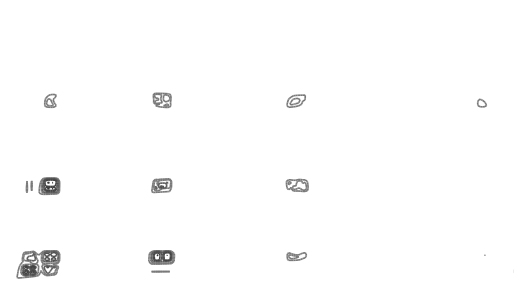

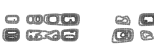

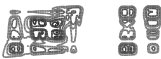


NOESY $\left(600 \mathrm{MHz}, \mathrm{CDCl}_{3}\right.$ ) of $\mathbf{1 3}$

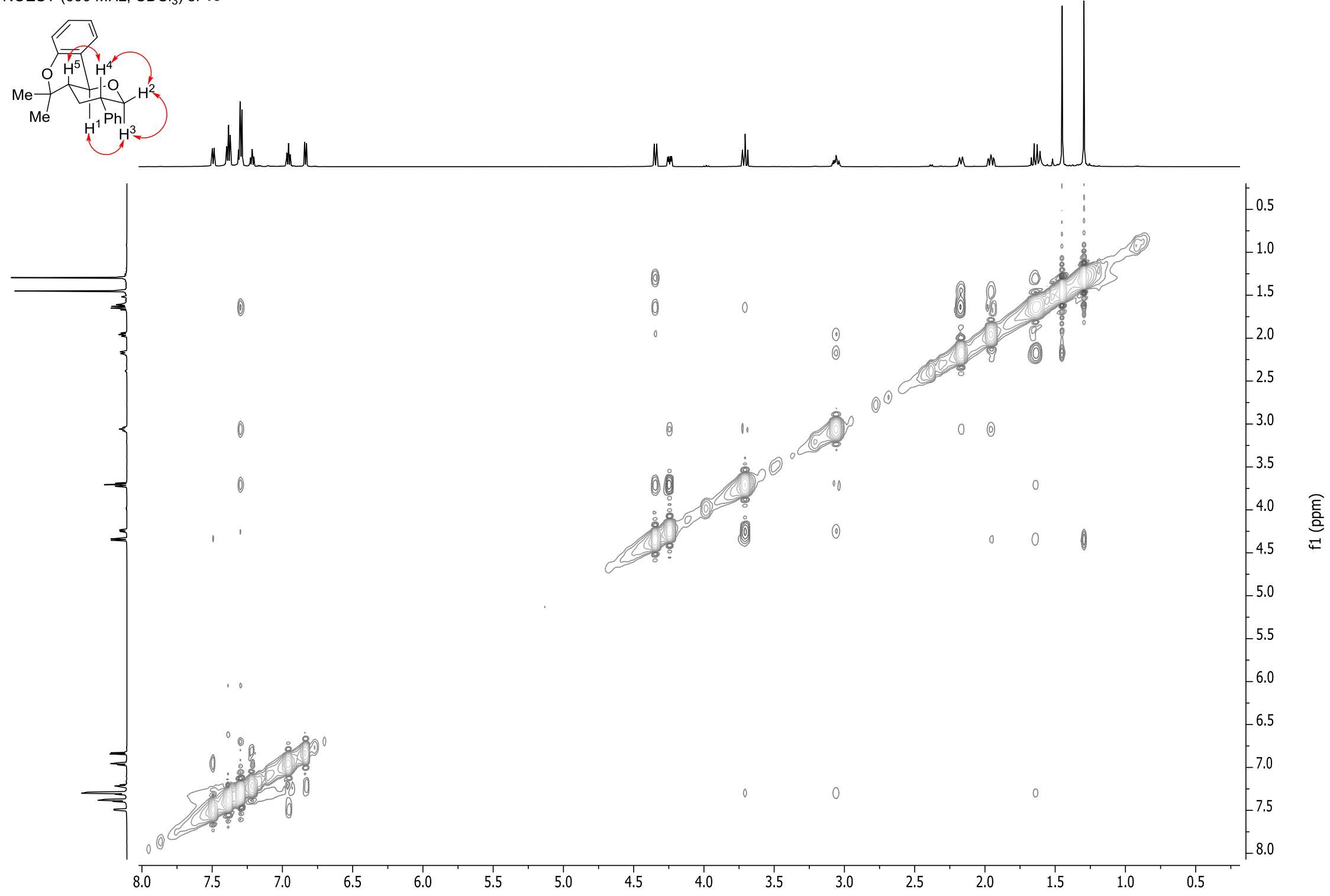




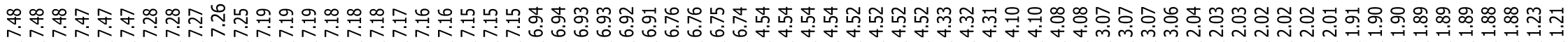

${ }^{1} \mathrm{H}-\mathrm{NMR}\left(600 \mathrm{MHz}, \mathrm{CDCl}_{3}\right)$ of 13 '
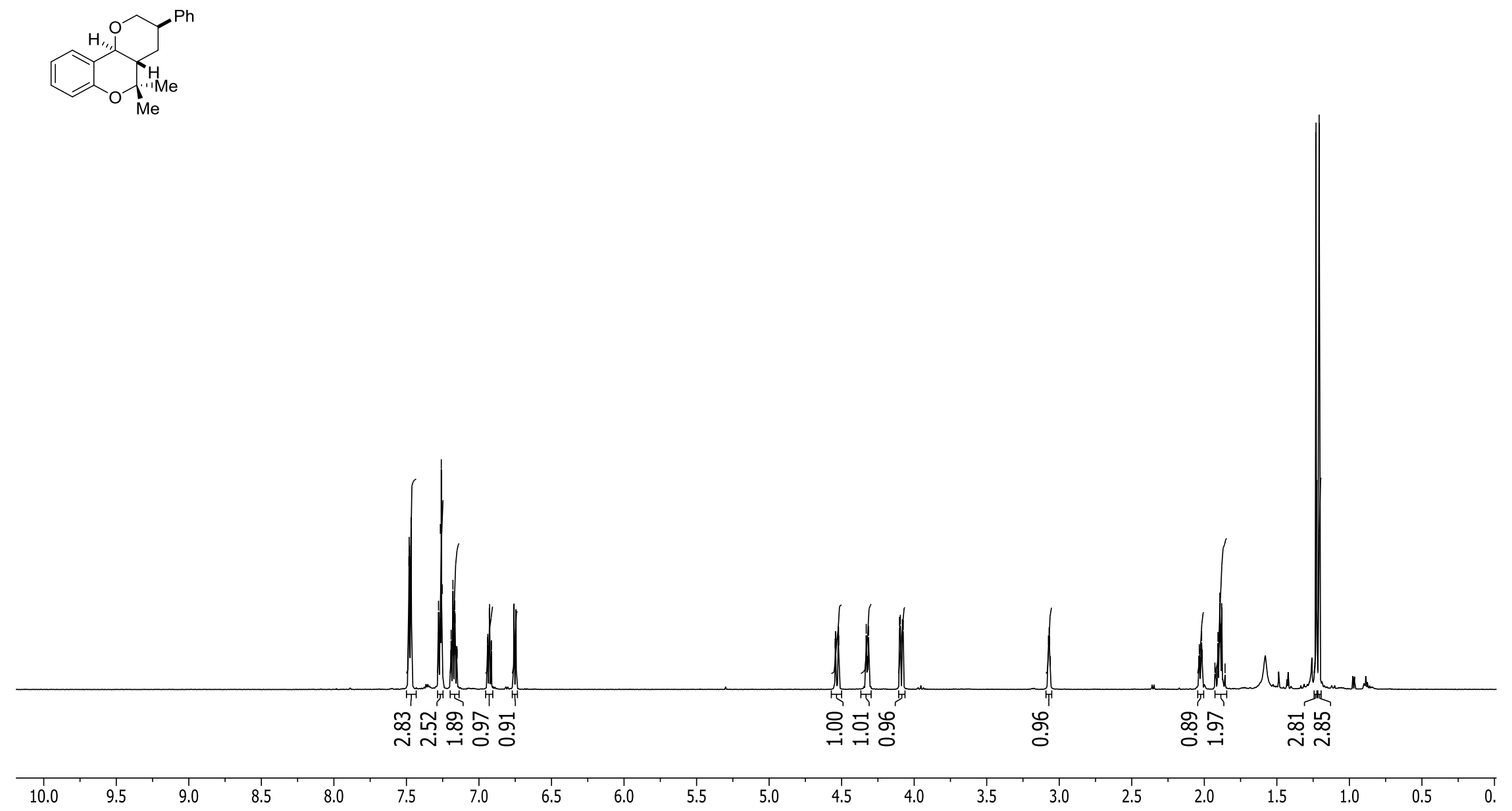


$$
\text { | }
$$

${ }^{13} \mathrm{C}-\mathrm{NMR}\left(150 \mathrm{MHz}, \mathrm{CDCl}_{3}\right)$ of $\mathbf{1 3}^{\prime}$
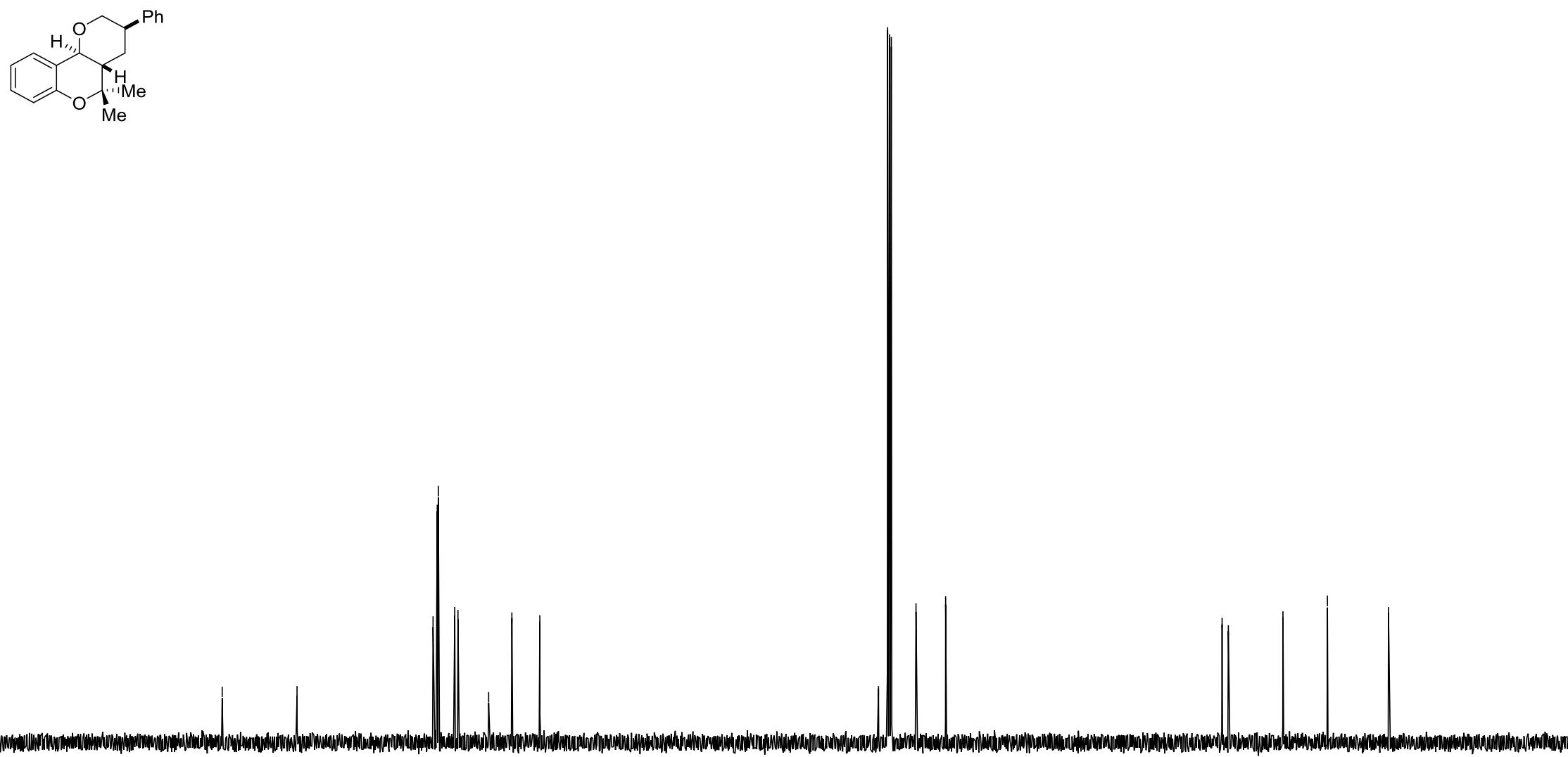

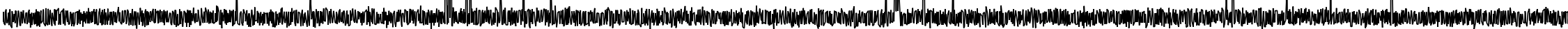

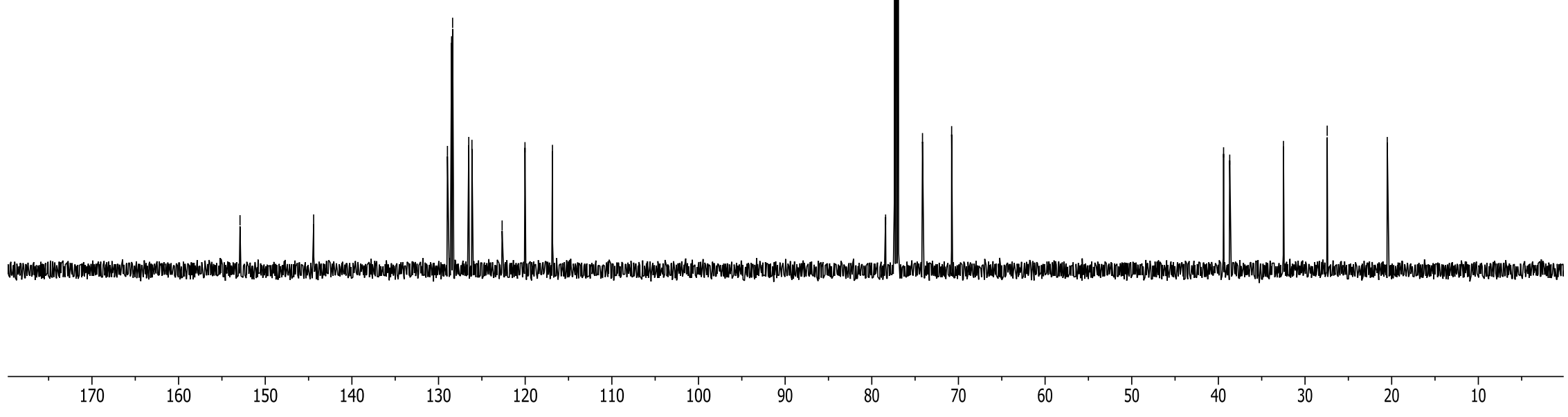




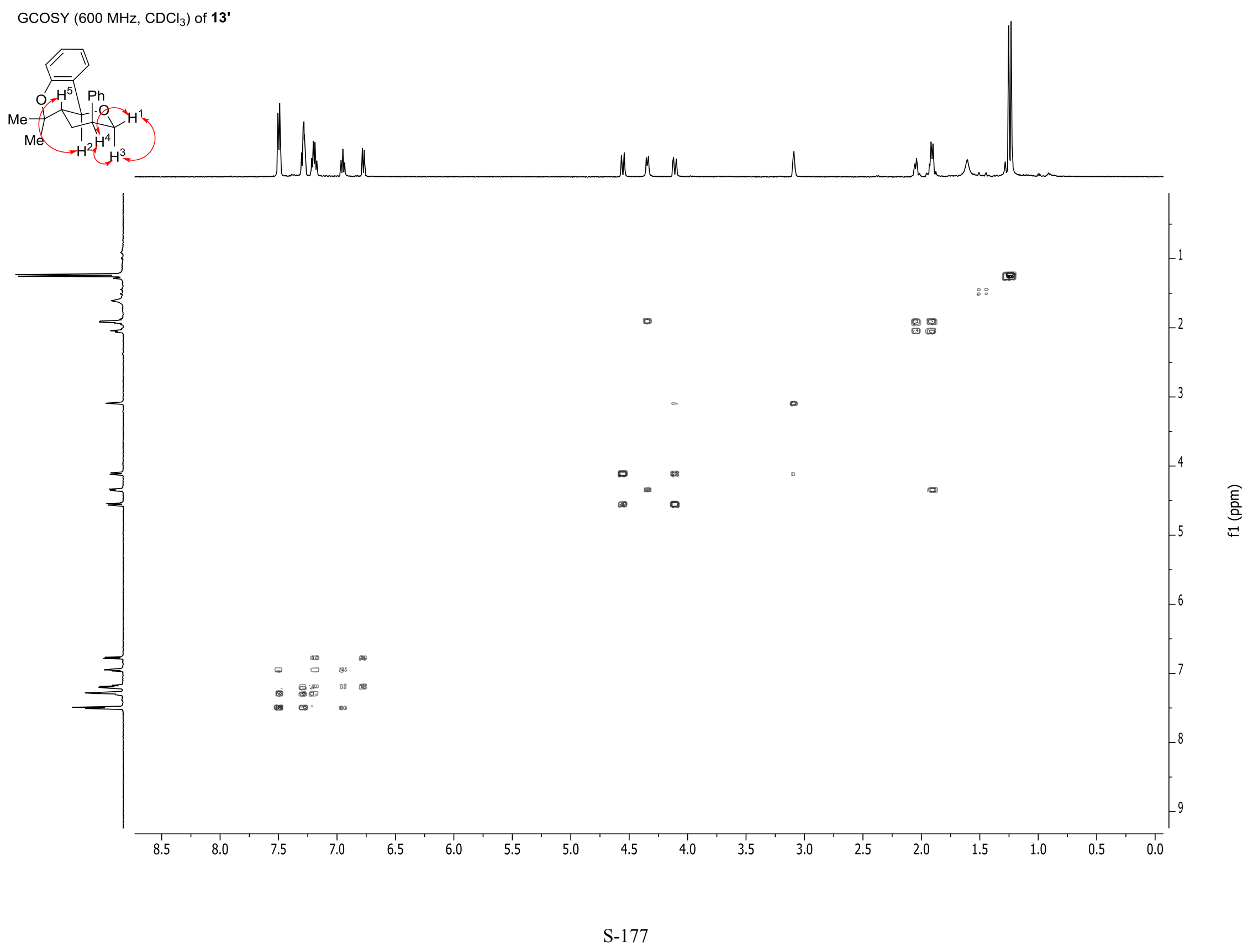


NOESY $\left(600 \mathrm{MHz}, \mathrm{CDCl}_{3}\right)$ of 13'

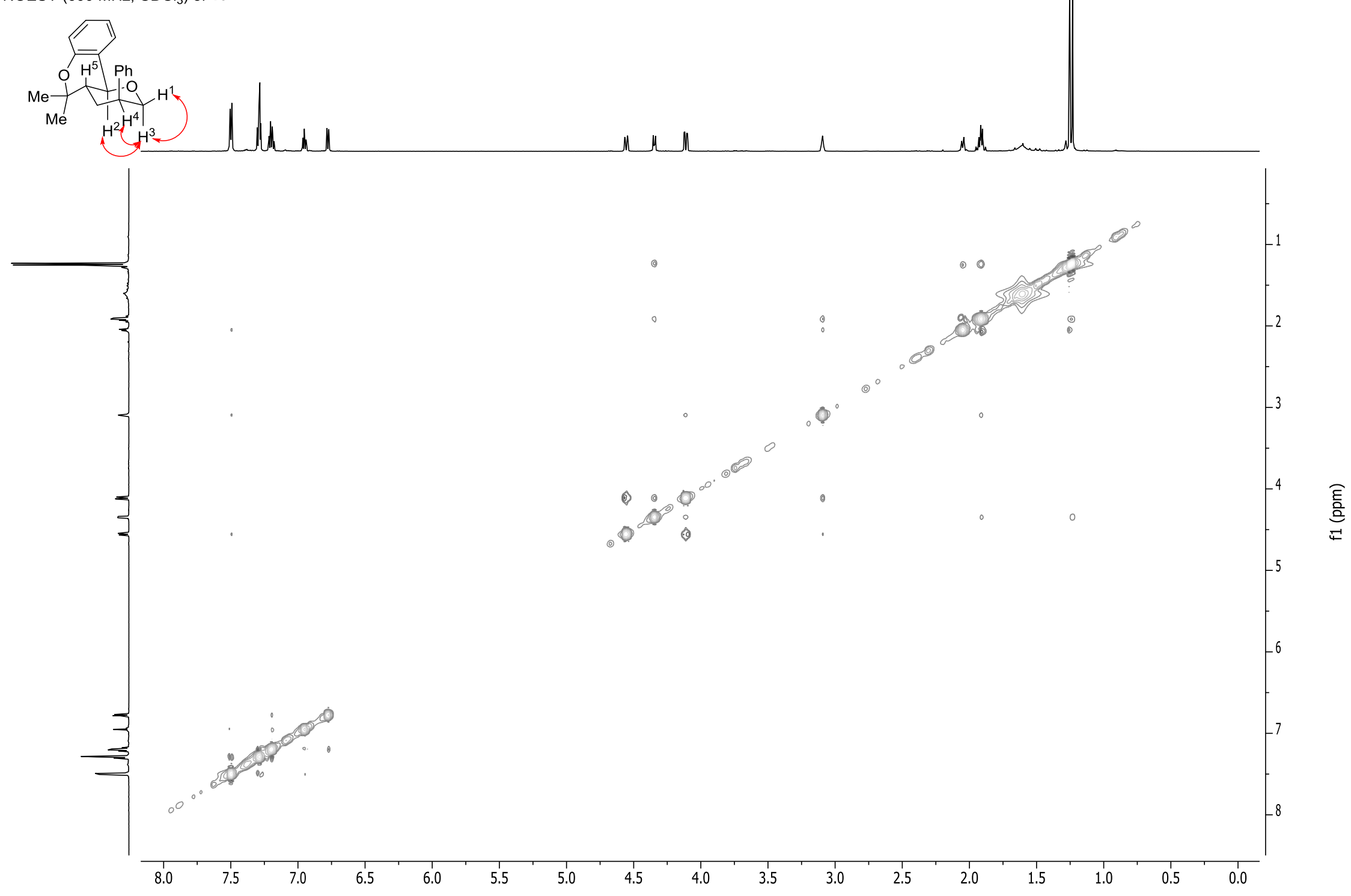




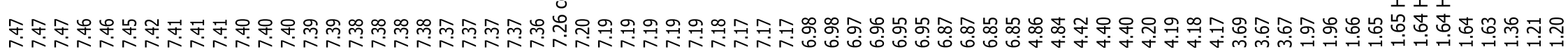
${ }^{1} \mathrm{H}-\mathrm{NMR}\left(500 \mathrm{MHz}, \mathrm{CDCl}_{3}\right)$ of $\mathbf{S 2}$
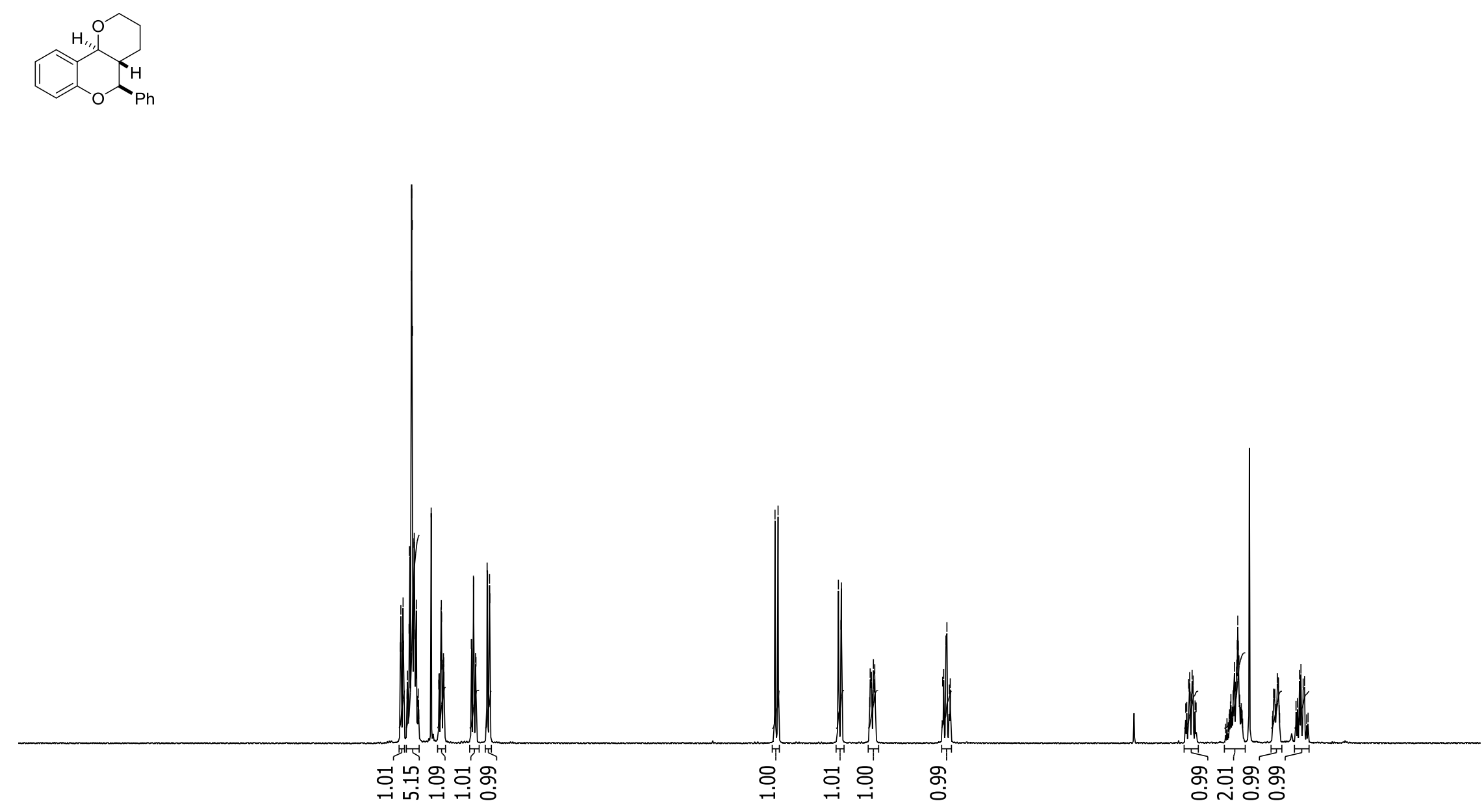


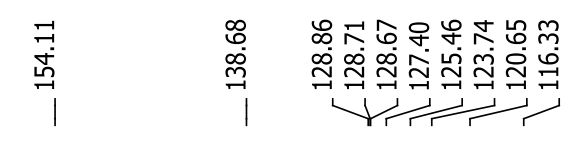

$\frac{m}{\bar{u}} \frac{m}{\bar{v}} \frac{m}{\mathrm{v}}$

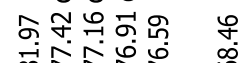

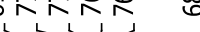

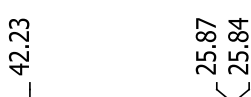

${ }^{13} \mathrm{C}-\mathrm{NMR}\left(125 \mathrm{MHz}, \mathrm{CDCl}_{3}\right)$ of S2
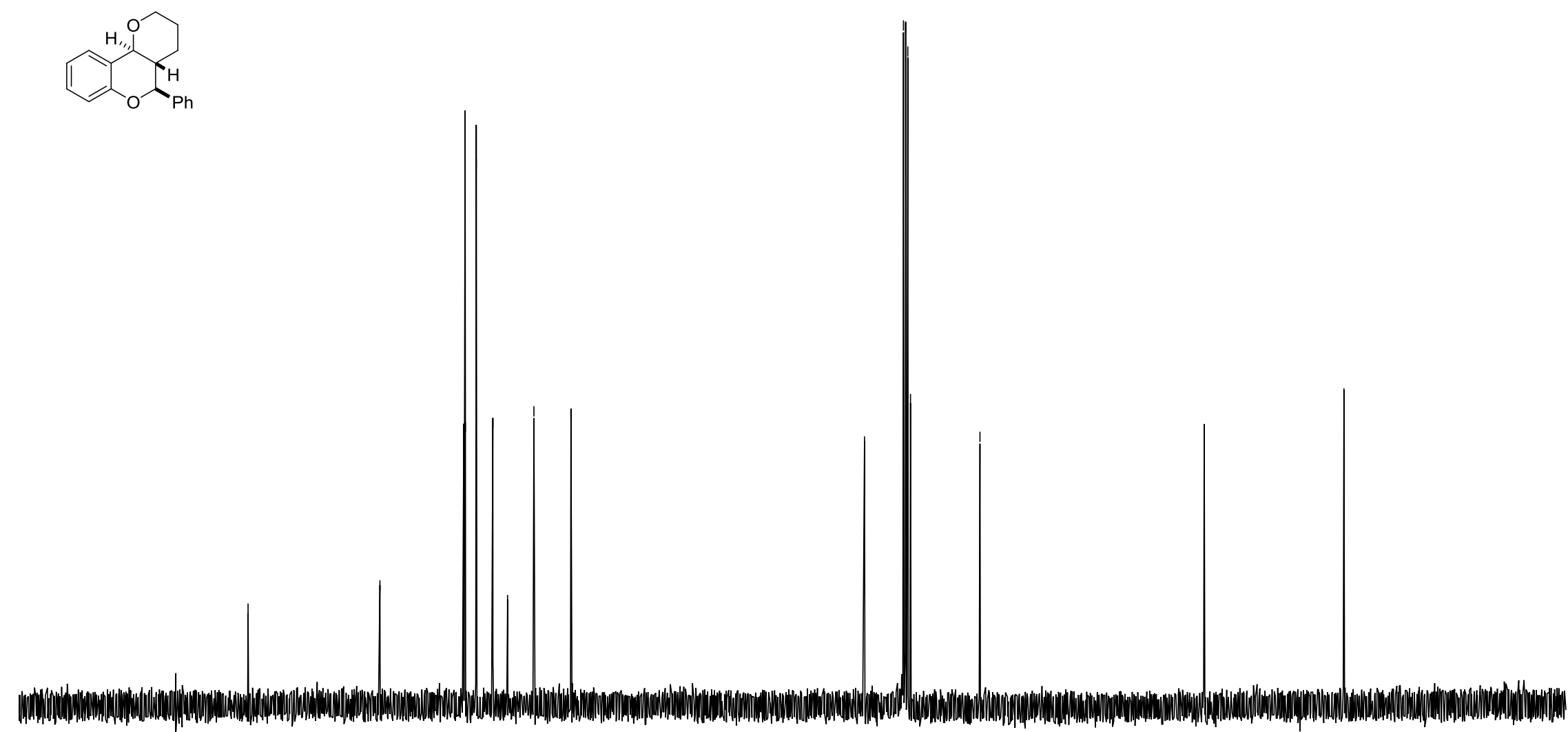

$\stackrel{7}{180}$

$170 \quad 160$

150

140

$130 \quad 120$

110

100

90

80

70

60

50

40

30

20

10 


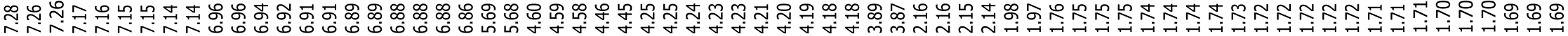

${ }^{1} \mathrm{H}-\mathrm{NMR}\left(600 \mathrm{MHz}, \mathrm{CDCl}_{3}\right)$ of $\mathbf{S} \mathbf{4}$

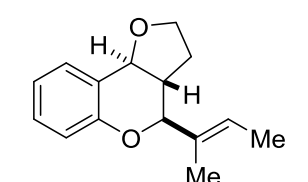

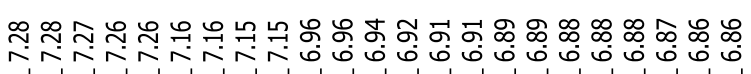
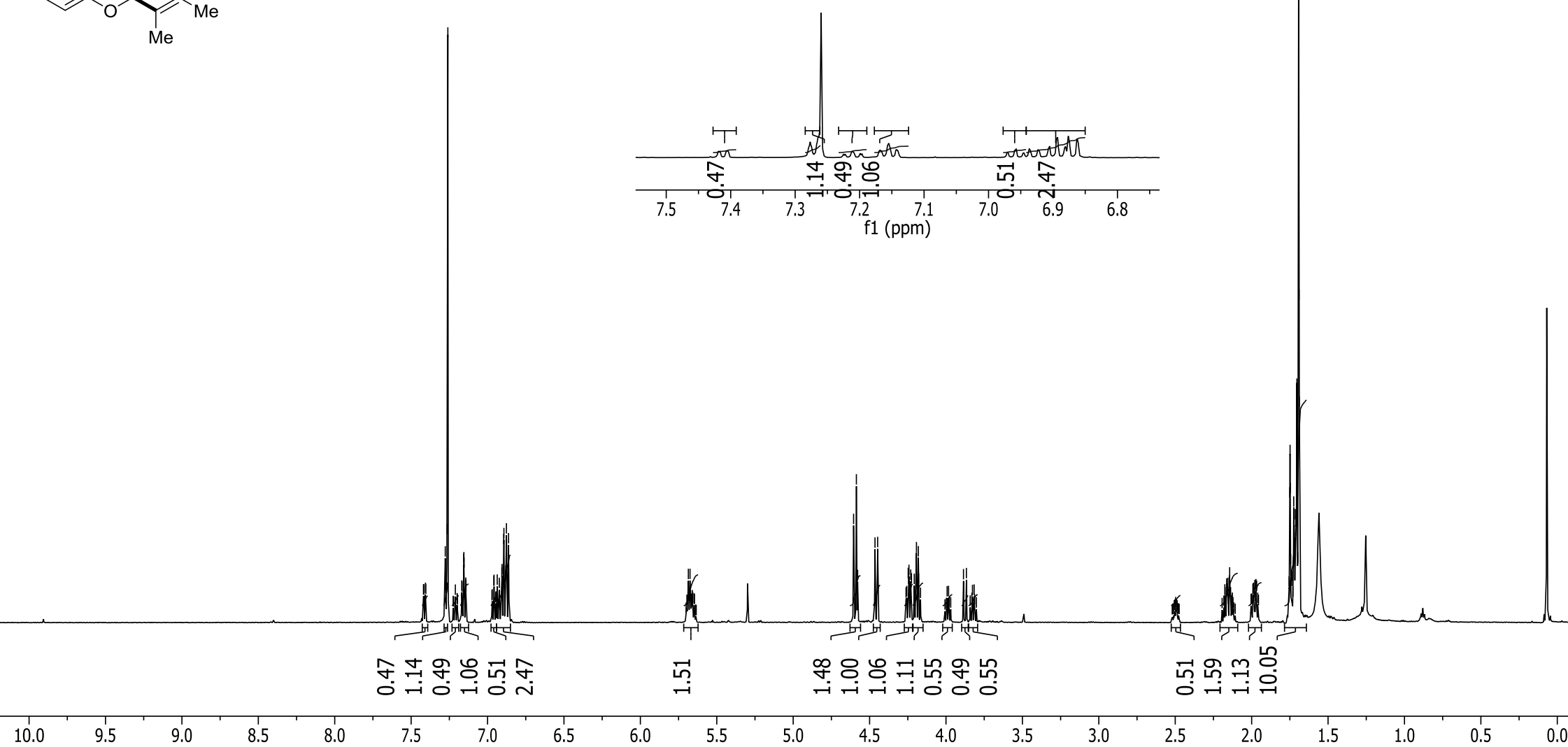


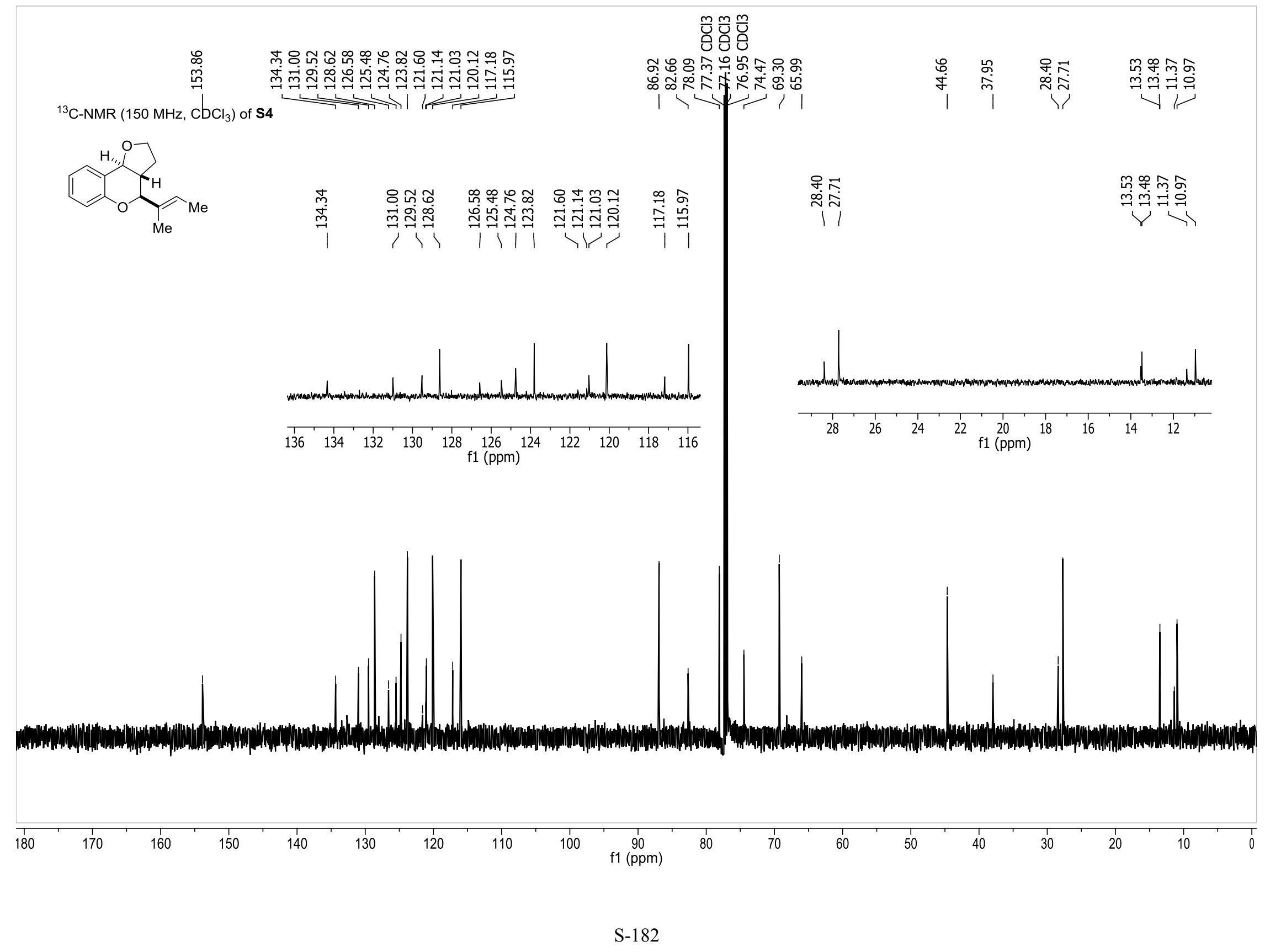

